\title{
Combined Experiment Phase II Data Characterization
}

Mark S. Miller, Derek E. Shipley, Teresa S. Young, Michael C. Robinson, and Marvin W. Luttges

University of Colorado

Boulder, Colorado

David A. Simms

National Renewable Energy Laboratory Golden, Colorado

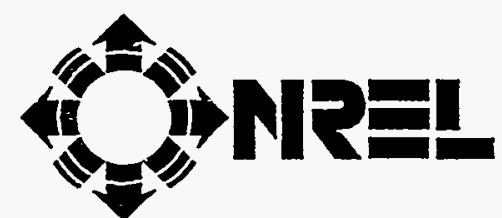

1617 Cole Boulevard Golden, Colorado 80401-3393

A national laboratory of the U.S. Department of Energy Managed by Midwest Research Institute for the U.S. Department of Energy Under Contract No. DE-AC36-83CH10093 


\section{Combined Experiment Phase II Data Characterization}

Mark S. Miller, Derek E. Shipley, Teresa S. Young, Michael C. Robinson, and Marvin W. Luttges

University of Colorado

Boulder, Colorado

David A. Simms

National Renewable Energy Laboratory

Golden, Colorado

NREL Technical Monitor:

David A. Simms

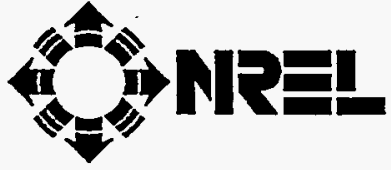

National Renewable Energy Laboratory 1617 Cole Boulevard Golden, Colorado 80401-3393

A national laboratory of the U.S. Department of Energy Managed by Midwest Research Institute for the U.S. Department of Energy under contract No. DE-AC36-83CH10093

Prepared under Subcontract

No. XAO-2-12236-01-103983

November 1995 


\section{NOTICE}

This report was prepared as an account of work sponsored by an agency of the United States government. Neither the United States government nor any agency thereof, nor any of their employees, makes any warranty, express or implied, or assumes any legal liability or responsibility for the accuracy, completeness, or usefulness of any information, apparatus, product, or process disclosed, or represents that its use would not infringe privately owned rights. Reference herein to any specific commercial product, process, or service by trade name, trademark, manufacturer, or otherwise does not necessarily constitute or imply its endorsement, recommendation, or favoring by the United States govemment or any agency thereof. The views and opinions of authors expressed herein do not necessarily state or reflect those of the United States government or any agency thereof.

Available to DOE and DOE contractors from:

Office of Scientific and Technical Information (OSTI)

P.O. Box 62

Oak Ridge, TN 37831

Prices available by calling (615) 576-8401

Available to the public from:

National Technical Information Service (NTIS)

U.S. Department of Commerce

5285 Port Royal Road

Springfield, VA 22161

(703) $487-4650$ 


\title{
COMBINED EXPERIMENT PHASE II DATA CHARACTERIZATION
}

\author{
Mark S. Miller, Derek E. Shipley, Teresa S. Young, Michael C. Robinson, Marvin W. Luttges \\ Department of Aerospace Engineering Sciences \\ University of Colorado, Boulder, CO 80309 \\ and \\ David A. Simms \\ National Renewable Energy Laboratory, Golden, CO 80401
}

\begin{abstract}
The National Renewable Energy Laboratory's "Combined Experiment" has yielded a large quantity of experimental data on the operation of a downwind horizontal axis wind turbine under field conditions. To fully utilize this valuable resource and identify particular episodes of interest, a number of databases were created that characterize individual data events and rotational cycles over a wide range of parameters. Each of the 59 five-minute data episodes collected during Phase II of the Combined Experiment have been characterized by the mean, minimum, maximum, and standard deviation of all data channels, except the blade surface pressures. Inflow condition, aerodynamic force coefficient, and minimum leading edge pressure coefficient databases have also been established, characterizing each of nearly 21,000 blade rotational cycles. In addition, a number of tools have been developed for searching these databases for particular episodes of interest. Due to their extensive size, only a portion of the episode characterization databases are included in an appendix, and examples of the cycle characterization databases are given. The search tools are discussed and the FORTRAN or C code for each is included in appendices.
\end{abstract}

\section{INTRODUCTION}

The National Renewable Energy Laboratory's (NREL's) "Combined Experiment" was established to provide a comprehensive test program focused on understanding the fundamental aerodynamics of rotating horizontal axis wind turbines (HAWTs). This fundamental aerodynamic understanding includes examining the difference between airfoil performance on an operational turbine and twodimensional wind tunnel tests, and determining the effects of the aerodynamics upon the turbine structure. To this end, NREL fully instrumented a turbine to obtain a wide range of inflow, aerodynamic, structural, and operational data. Phase II of the Combined Experiment has been completed using untwisted, untapered blades. Nearly 300 minutes of high frequency data has been collected.

Because of the large amount of data collected during the experiment, characterizing the data is necessary to aid in the location of pertinent data. Two primary methods of characterization have been employed. First, the mean and standard deviation values for all data channels were calculated for every data episode. Each episode is five minutes in length. The statistical summary values aid researchers in determining what data is available over large periods of time. For instance, researchers using ADAMS/WT (Automatic Dynamic Analysis of Mechanical Systems/Wind Turbine) might find data episode characterizing useful. However, to understand factors such as the turbine's aerodynamics, a second, more in-depth method of characterizing the data is required. This method examines the data on an individual cycle basis. A cycle is defined as a single rotation of the instrumented blade from $0^{\circ}$ azimuth, the instrumented blade straight up, to $360^{\circ}$ azimuth. This second method characterized each rotational cycle by inflow conditions, aerodynamic force coefficients, and minimum leading edge pressure coefficient.

\section{TEST SETUP}

NREL's Combined Experiment horizontal axis wind turbine is a 10.1 meter diameter, three-bladed machine that rotates at a constant 72 revolutions per minute (RPM) and is capable of producing 20 kilowatts $(\mathrm{kW})$ of power (Figure 1). The turbine is supported on a 0.4 meter $(\mathrm{m})$ cylindrical tower at a height of 17 meters from the ground to the center of the hub. The blades used were rectangular, untwisted NREL S809 airfoil sections with an 0.457 meter chord.

One of the three blades was completely instrumented with pressure transducers (Figure 2) at four different span locations. Unsteady pressure data from all transducers were collected simultaneously. The data 
sample rate (521 hertz [Hz]) was sufficient to capture the dynamic and transient pressure events elicited from time variant inlet flow conditions.

The inlet flow conditions were measured by the vertical plane array located 12 meters upwind of the turbine. The inlet flow magnitude and direction across the turbine diameter was measured using 11 prop-vane and 2 bi-vane anemometers. Eight of the prop-vane anemometers were arranged in a 4 meter radius circle approximately 16 meters above the ground. The remaining anemometers were spaced evenly inside the circle in a vertical line. The two bivane anemometers were mounted outside the circle on the horizontal axis.

Strain gages mounted on the blades and low-speed shaft were used to measure the rotor's mechanical loads. Torsional and edgewise bending moments were measured at the root of the instrumented blade and at two spanwise locations. Flapwise bending moments were measured at the roots of all three blades and at five spanwise locations on the instrumented blade (Figure 2). Mechanical turbine power was determined from measured low-speed shaft torque and rotor speed. Electrical power was measured via a power watt transducer connected to the three-phase, 480 volt, alternating current induction generator.

Data was collected over a wide range of nominal operating conditions for the turbine. Each data run consisted of a five-minute continuous data collection episode wherein all data were stored on time encoded tape. The turbine blade rotated at a nominal $1.2 \mathrm{~Hz}$, thus, typically 360 consecutive blade rotation cycles are contained in each five-minute episode. A total of 59 episodes consisting of 239 channels of data were collected representing approximately 21,000 complete rotation cycles of the instrumented turbine blade. The 59-episode data set consists of 9 gigabytes of binary data and requires 30 optical disks for storage. For more information on the test setup and the data collected, refer to Butterfield et al. (1992) and Miller et al. (1994).

\section{DATA EPISODE CHARACTERIZATION}

Analyzing a data set of this size and complexity is no trivial task. The systematic identification of data episodes of interest demands detailed knowledge of the data recorded to each optical disk. Summary files were created for each of the 59 five-minute episodes that consist of the mean, standard deviation, minimum, and maximum for all 239 data channels.
These summary files are recorded along with their respective data files on optical disk.

The summary file data is very helpful when investigating a single data episode, but even more useful is the ability to examine a certain parameter across all of the data episodes. Thus, databases were created which allow for examination of a single parameter. These databases are characterized based upon the summary statistics and contain mean, average, maximum, and minimum values for selected channels. All data channels, except for the blade surface pressures, have been characterized in this manner, but all are not included due to size constraints. Appendix A contains the tables most frequently used by the authors.

These databases can be utilized in a number of ways. For instance, using data from the yaw and velocity (disc-averaged wind speed) tables in Appendix A, Figure 3 gives an indication of the mean inflow conditions for all the data episodes. The inflow conditions, velocity and yaw, are the independent variables which drive the turbine's aerodynamics and, thus, its structural response. Figure 3 indicates that a significant portion, 45 of 59 , of the data episodes have an average yaw between $-9^{\circ}$ and $3^{\circ}$ and an average velocity between 5 and 17 meters per second $(\mathrm{m} / \mathrm{s})$. The low yaw angles are characteristic of downwind HAWTs since they tend to align themselves with the wind direction. Note, however, that there are large gaps in the matrix. For instance, there is only one data episode, d069021, with a mean yaw between $9^{\circ}$ and $15^{\circ}$. Figure 4 depicts the same matrix, but the mean and standard deviation values for the inflow conditions have been inserted. The top chart provides mean velocity and standard deviation while the lower chart has mean yaw and standard deviation. As an example, looking at Figure 3, there is only one data episode, d072032, that meets the conditions of $-15^{\circ}$ to $-9^{\circ}$ yaw and 8 to $11 \mathrm{~m} / \mathrm{s}$ in velocity. Figure 4 shows that this episode has an average velocity of $10.3 \pm 3.0 \mathrm{~m} / \mathrm{s}$ and an average yaw of $-9.4^{\circ} \pm 21.3^{\circ}$. In boxes containing more than one data episode, all the data episodes are included in the average and standard deviation. In addition, the column and row averages are calculated along with a total for all the data episodes. The average velocity and yaw for all data episodes, $10.1 \pm 4.2 \mathrm{~m} / \mathrm{s}$ and $2.4^{\circ} \pm 3.3^{\circ}$ respectively, also provide an indication of where most of the data are located. 


\section{ROTATIONAL CYCLE CHARACTERIZATION}

The utility of these episode summary files in understanding turbine aerodynamics is severely limited due to the highly transient nature of the experimental data. As Figure 5 illustrates, the distribution of inflow velocity for two different episodes can be significantly different, even though they have similar means and standard deviations. Neither of these velocity distribution plots shows a stochastically normal distribution, nor are the shapes of the velocity distributions similar. Thus, to examine certain turbine aspects, a much finer resolution of data categorization was required.

Previous Combined Experiment investigators binned large amounts of the data on certain characteristics, such as inflow conditions (Huyer, 1993). Although this method provided some insight into turbine performance, to achieve the goal of understanding the turbine's fundamental aerodynamics and structural response, a more in-depth examination is required. Thorough examination of the Combined Experiment data requires categorization on a individual cycle basis. Thus, databases were created to contain average values and standard deviations of certain parameters for each of the 21,000 rotational cycles. Three separate databases were established based upon inflow conditions, aerodynamic force coefficients, and minimum leading-edge pressure coefficients.

\section{Inflow Conditions Database}

The inflow conditions, velocity and yaw, are extremely important when examining turbine data because these two independent variables drive the turbine's aerodynamics and have a significant effect upon structural responses. The program used to create the inflow conditions database, CYCCAT.C, is located in Appendix B along with the necessary header files. This program outputs cycle number along with the mean and standard deviation values of velocity $(\mathrm{m} / \mathrm{s})$, yaw (degrees [deg]), horizontal shear $((\Delta \mathrm{m} / \mathrm{s}) / \mathrm{m})$, and vertical shear $((\Delta \mathrm{m} / \mathrm{s}) / \mathrm{m})$ for each cycle within each data episode. The velocity value is obtained from the disc averaged wind speed. The disc averaged wind speed is calculated by averaging together readings of the eight prop-vane anemometers arranged in a $4 \mathrm{~m}$ radius circle on the Vertical Plane Array (VPA). The yaw value is created by subtracting the hub height wind direction on the VPA from the turbine yaw angle. Thus, yaw is the angle between the turbine and the wind direction. The horizontal shear is the difference between the value recorded by the prop-vane anemometers located at the 3 and 9 o'clock positions on the outer anemometer circle divided by the distance between them, 10.1 meters. The vertical shear is similar, except the anemometers at 12 and 6 $o$ 'clock are used. However, in reality the distance between the anemometers is 8.0 meters. Thus, the baseline data for horizontal and vertical shear is incorrect. The data can be corrected by multiplying the shear data by 10.1/8.0.

The program requires the user to input the name of the directory containing the data episode header file and the header file name. The program then creates the inflow conditions database. The program generates two output files for each data episode. For example, if data episode $\mathrm{d} 073011$ is being processed, the files d073011.sum and d073011.out are generated. The d073011.sum file contains the data in the form shown in Table 1. The d073011.out file contains only raw data, without the various titles (Table 2). For the Phase II data, the instrumented blade was at approximately $180^{\circ}$ azimuth for all the data episodes. Thus, the first rotational cycle only contains information about half a rotational cycle. The inflow conditions database did not record these values, although the other databases do, because these half cycles were not of interest to the authors.

To simplify the explanation of the files, a $*$ will be used in place of a data episode name. The primary usage of the *.sum files is for visual inspection of cycle inflow conditions. The *.out files are best suited for use with computational search tools. Using these files, the distribution of all available data on a individual cycle basis was generated (Figure 6). As earlier predicted, most of the cycles are located around $0^{\circ}$ yaw and are between 5 and $17 \mathrm{~m} / \mathrm{s}$. However, using the data episode averaged data, it appeared that there was very little data for conditions where the yaw was more than $9^{\circ}$. Examining the data on a individual cycle basis, it is clear that the earlier conclusion is untrue because there is a significant amount of data for this range at all velocities.

A variety of search tools have been written to analyze the inflow conditions database. One of these programs, BESTCYC.F, is located in Appendix C. The program uses all the *.out files created by CYCCAT.C as input. As output, the program generates a file that contains the data episode, cycle number, and the mean and standard deviation values for velocity and yaw. The user is able to select from three different options. The first option simply finds 
the mean and standard deviation in velocity and yaw for a user-provided range of cycles on a given episode. The second option locates a user-specified number of consecutive cycles on each episode having the lowest standard deviation in velocity or yaw. The third option allows the user to select a group of consecutive cycles based on the mean velocity and/or yaw limits defined by the user. The program then finds all the data that meets the input criteria. For example, this option allows the user to find all groups of 50 consecutive cycles that have a mean velocity between 9.5 and $10.5 \mathrm{~m} / \mathrm{s}$ and a mean yaw between $-2.5^{\circ}$ and $2.5^{\circ}$. The user can then visually inspect the list or create a program to do it for them. This enables groups of cycles with low standard deviations in velocity and high standard deviations in yaw to be identified

\section{Aerodynamic Force Coefficients Database}

The authors decided through examination of the Combined Experiment data that the best means of determining aerodynamic performance was by the normal force coefficient, $C_{n}$, and the tangent force coefficient, $C_{t}$ (Miller et al., 1994). The normal and tangent force coefficients were calculated for each of the four primary span locations $(30 \%, 47 \%, 63 \%$, and $80 \%$ span). These forces were created by integrating the pressure coefficient, $C_{p}$, values over the entire blade section during the initial Combined Experiment data processing. The program used to create the aerodynamic force coefficients database, AVCNCT.C, is located in Appendix D along with the necessary header files.

This program outputs cycle number along with the mean and standard deviation values of the normal and tangential aerodynamic force coefficients for the four primary span locations for each data episode. In addition, AVCNCT.C outputs the maximum value of both coefficients at the primary span locations for each cycle. This can be very useful in determining which cycles have significant aerodynamic forces that may be damaging the turbine structure.

The program requires the user to input the name of the directory containing the data episode header file and the header file name. After these have been entered, the program creates the aerodynamic force coefficients database. As in CYCCAT.C, each side of the 30 optical disks must be processed to create the entire database. However, the program is different from CYCCAT.C in several ways. The first major difference is that while creating the database, AVCNCT.C also checks and corrects errors in the aerodynamic force coefficients. Second, this program finds the maximum normal and tangent force coefficients at each of the four primary span locations for each individual cycle. Third, the program creates seven output files.

The program checks for errors on an individual data point basis. The exact reason for the few errors has not been determined, but they appear to be due to rare data glitches. The program calculates the standard deviation of the normal force coefficients for an entire cycle. Then, if a data point is more than two standard deviations away from the previous point, the data is considered to be anomalous. Anomalous points are corrected by replacing the anomalous value with the average of the two preceding points. Only the normal force coefficient data was examined. Therefore, if a normal force coefficient point is changed, the corresponding tangent force coefficient is also changed. This scheme was implemented because the tangent force values are very small, making this method of correction difficult and sometimes incorrect.

AVCNCT.C generates seven output files for each data episode. If data episode d073011 is being processed, d073011cn.sum, d073011ct.sum, d073011max.sum, d073011.err, d073011cn.out, d073011ct.out, and d073011max.out are created. The *cn.sum files contain all the normal force coefficient data (Table 3 ), the *ct.sum files contain all the tangent force coefficient data (Table 4), and the *max.sum contains all the maximum normal and tangent force coefficient data (Table 5). The * cn.out, *ct.out, and ${ }^{*}$ max.out files simply contain the same data as the corresponding *.sum files without the titles. The *.err files show the value of the anomalous points and the value after being corrected (Table 6).

Several programs have been written to analyze the aerodynamic force coefficients database. One of these programs, CNCTMAX.F, is located in Appendix E. This program not only searches the aerodynamic force coefficients database, but examines the inflow conditions database as well. Thus, the program requires the *.out files created by CYCCAT.C and the ${ }^{*} \mathrm{cn}$.out, *ct.out, and ${ }^{*}$ max.out files created by AVCNCT.C. The program ranks every cycle by mean normal and tangent force coefficients in decreasing order for a user-specified span location. The user must input whether normal or tangent force coefficient is to be examined, the span location desired, the number of values to be 
written to an output file, and the name of the output file. For instance, the program can be used to obtain the fifteen cycles with the highest tangent force coefficients at $47 \%$ span. The program outputs rank, data episode name, cycle number, mean force coefficient and standard deviation, the maximum force coefficient in that cycle, the mean angle of attack for the cycle (deg), the disc-averaged wind speed mean and standard deviation $(\mathrm{m} / \mathrm{s})$, and the yaw mean and standard deviation (deg). An example of the output is located in Table 7. The mean angle of attack for the cycle is calculated using geometric relations by methods of Shipley et al. (1994). The rest of the data is obtained from either the aerodynamic force coefficients or the inflow conditions database files.

A significant shortcoming of the present database is the inexact dynamic pressure that is used to create the normal and tangent force coefficients. This dynamic pressure is not yaw dependent and, thus, can be inaccurate during yawed conditions. However, a yaw dependent dynamic pressure can be derived using several different methods (Shipley et al., 1994). These methods were developed after the categorization program, AVCNCT.C, and the organizational program, CNCTMAX.F, were written.

\section{Minimum Leading-Edge Pressure Coefficient} Database

The minimum leading-edge pressure coefficient database was established to aid in the detection of dynamic stall events. One of the signatures of dynamic stall is a large suction peak or, in other words, a peak in the minimum leading-edge pressure coefficient. The program used to create the minimum leading-edge pressure coefficients database, DSTALLTAPE.F, is located in Appendix F. This program outputs the data episode and cycle number along with the minimum leading edge pressure coefficient for the cycle and the span location of the occurrence $(30 \%, 47 \%, 63 \%$, or $80 \%$ span). Also included is the disc-averaged wind speed $(\mathrm{m} / \mathrm{s})$, yaw (deg), and azimuth angle (deg) values at the precise moment when the minimum leading edge pressure coefficient occurred.

The program requires the user to input the name of the episode data file and output file name. In addition, the program allows the user to decide whether or not to renormalize the $C_{p}$ values by a yaw dependent dynamic pressure. The dynamic pressure used is based upon a geometric model described in Shipley et al. (1994). An example of a database created by DSTALLFILE.F is located in Table 8. A header is included in the output files to indicate the contents. The authors' convention for naming the minimum leading edge pressure coefficient was maxcp?????r.dat. For example, for data episode d075022 the database was named maxcp75022r.dat. The ' $r$ ' in the name indicates that the $C_{p}$ values were renormalized.

One of the programs created to analyze this database, DSTALL.F, is included in Appendix G. This program allows the user to limit the data in a number of ways: by minimum leading edge pressure coefficient, by span location, by velocity, by yaw, and by azimuth angle. These options permit the analysis of dynamic stall occurrences and magnitude under different operational conditions. For example, limiting the data below a given $C_{p}$ allows the user to examine the data for all $C_{p}$ spikes less than -8 . Excluding an azimuthal region allows for a quantification of dynamic stall occurrence during different regions of the cycle. The program also allows for determination of occurrence of dynamic stall at more than one span location. Additionally, the program will display the number of cycles that fit the user-specified criteria. This feature allows the user to determine if the criteria is too general or too specific.

DSTALL.F is also able to present the data in several different formats. The data can be sorted by velocity, yaw, or minimum leading-edge pressure coefficient. The data can also be binned by velocity and yaw by specifying the upper and lower velocity and yaw bin limits along with the bin width. This final option averages all the $C_{p}$ values of the cycles meeting the constraints set by the user. For instance, for a certain velocity range the user may wish to know the average $C_{p}$ value for $30 \%$ span if the $C_{p}$ value is limited to less than -10 . The program generates an output file with the same format as the minimum leading-edge pressure coefficients database (Table 8).

The data obtained from the minimum leading-edge pressure coefficient database has a variety of applications. For example, the data could be used to obtain the frequency of dynamic stall occurrence under various inflow conditions (Figure 7). The criteria established to identify dynamic stall was that the $C_{p}$ was less than -10 .

\section{CONCLUSION}

In order to examine the massive amount of data collected during Phase II of the Combined 
Experiment, the data has been characterized by data episode and by individual cycles. Also, a number of useful programs have been written not only to create the databases, but to examine them as well. Future researchers may find these programs useful when examining Phase II data or even for advanced phases of the Combined Experiment.

The various databases, the programs used to create them, and the search tools can be obtained from the authors.

\section{REFERENCES}

Butterfield, C.P., W.P. Musial, and D.A. Simms (1992): "Combined Experiment Phase I Final Report", NREL/TP-257-4655, National Renewable Energy Laboratory, Golden, CO.

Huyer, S. (1993): "Examination of Forced Unsteady Separated Flow Fields on a Rotating Wind Turbine Blade", NREL/TP-442-4864, National Renewable Energy Laboratory, Golden, CO.

Miller, M.S., D.E. Shipley, , M.C. Robinson, M.W. Luttges, and D.A. Simms (1994): "Determination of Data Reliability for Phase II of the Combined Experiment", NREL/TP-442-6914, National Renewable Energy Laboratory, Golden, $\mathrm{CO}$.

Shipley, D.E., M.S. Miller, M.C. Robinson, M.W. Luttges, and D.A. Simms (1994): "Techniques for the Determination of Local Dynamic Pressure and Angle of Attack on a Horizontal Axis Wind Turbine", NREL/TP-442-7393, National Renewable Energy Laboratory, Golden, CO.

APPENDIX TABLE OF CONTENTS

Appendix A: Single Parameter Tables for Entire Data Episodes

Appendix B: CYCCAT.C, BEN_IO.C, READ.C, MAIN.H, MAKEFILE, LAST_BIN.DAT

Appendix C: BESTCYC.F

Appendix D: AVCNCT.C, DIRECTORY.DAT, MAKEFILE, LAST_BIN.DAT

Appendix E: CNCTMAX.F

Appendix F: DSTALLTAPE.F

Appendix G: DSTALL.F 


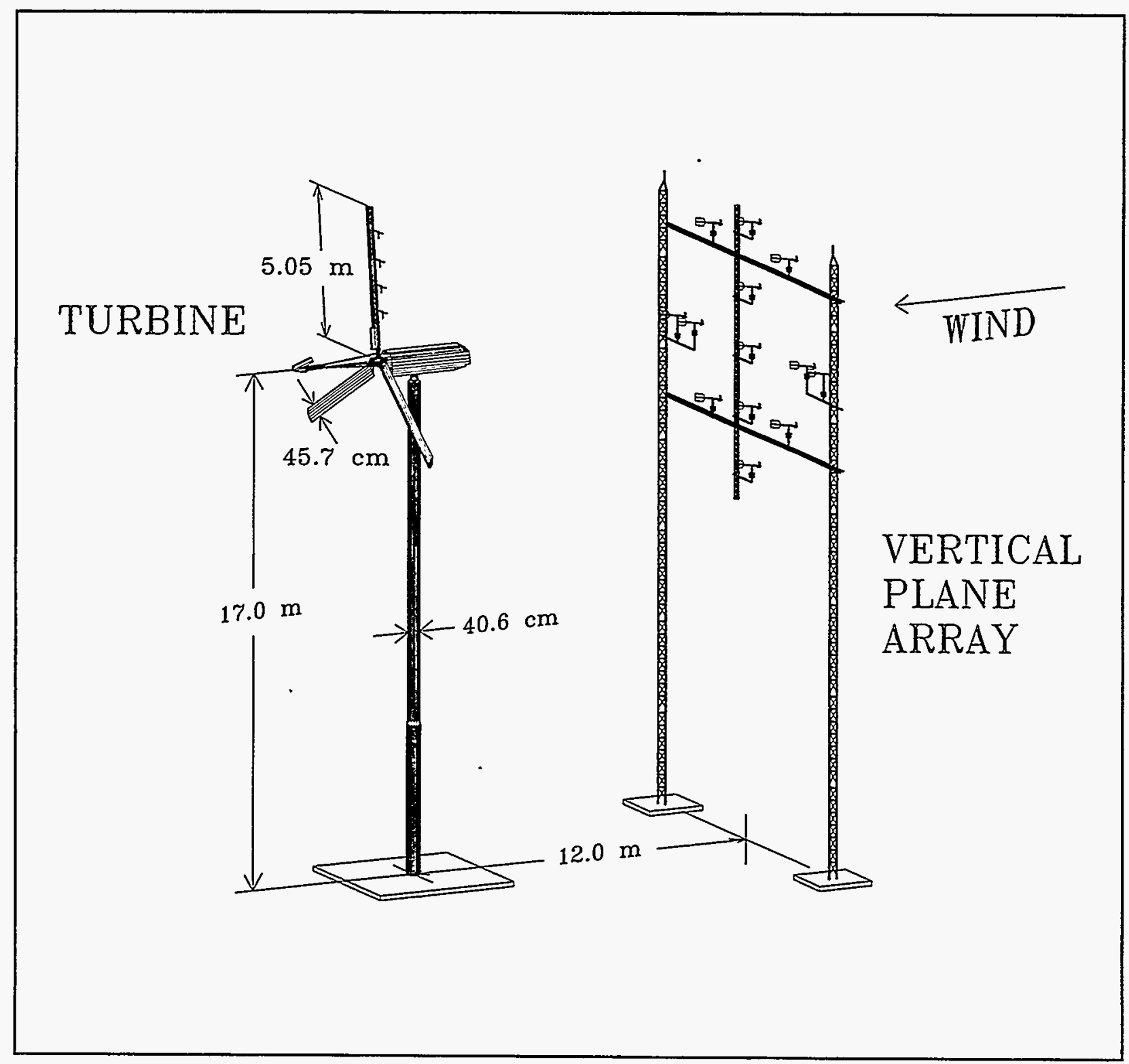

FIGURE 1. VIEW OF THE COMBINED EXPERIMENT TEST SITE INCLUDING THE GRUMMAN WIND STREAM 33 HORIZONTAL AXIS WIND TURBINE AND THE VERTICAL PLANE ARRAY. 


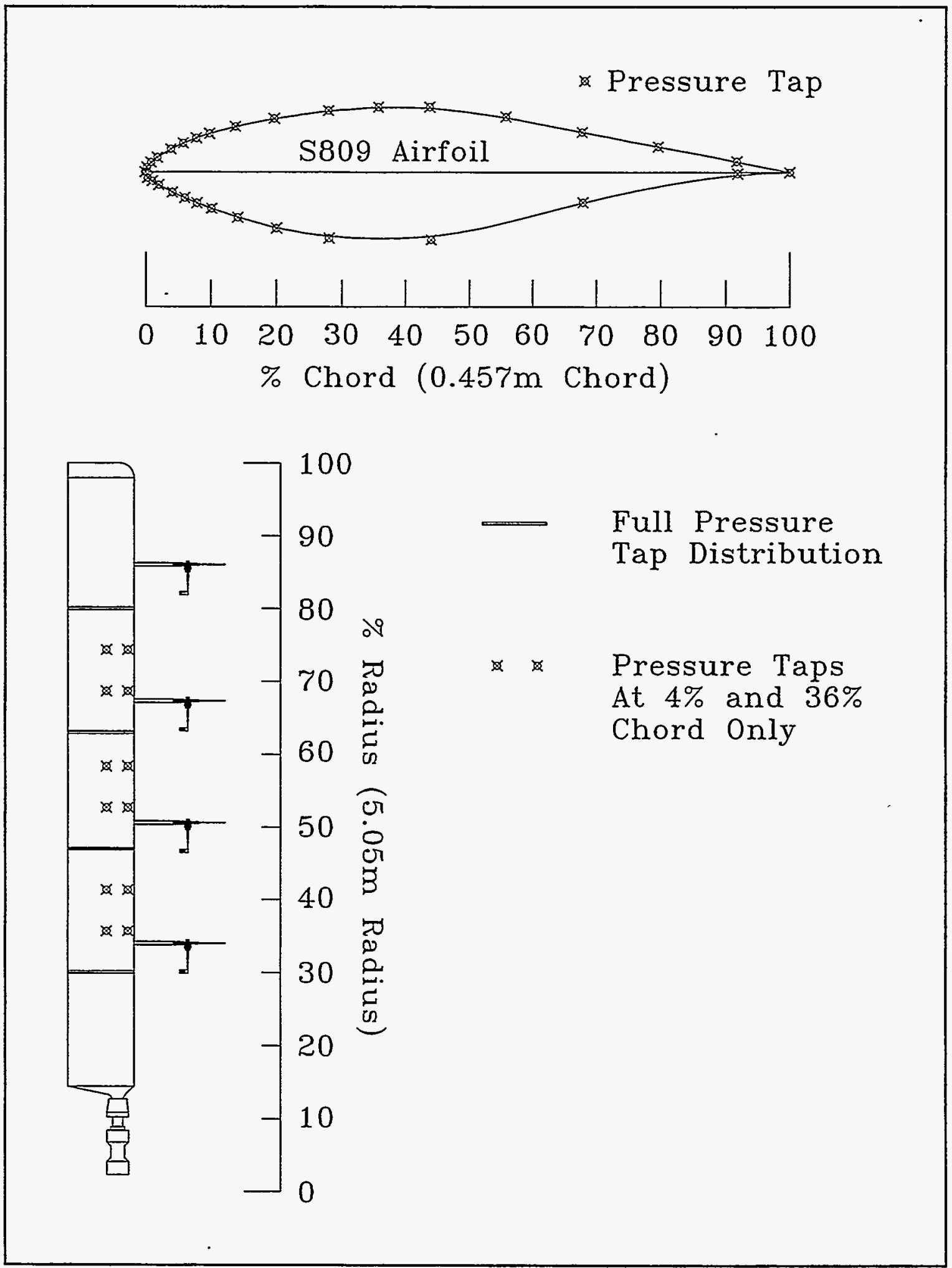

FIGURE 2. ROTOR BLADE CROSS SECTION AND LONGITUDINAL VIEWS SHOWING CHORDWISE PRESSURE TAP DISTRIBUTION AT EACH OF FOUR PRIMARY LOCATIONS $(30 \%, 47 \%, 63 \%$, AND $80 \%$ SPAN). DYNAMIC PRESSURE PROBES AND ANGLE OF ATTACK FLAGS ARE LOCATED JUST OUTBOARD OF SURFACE PRESSURE TAPS. 


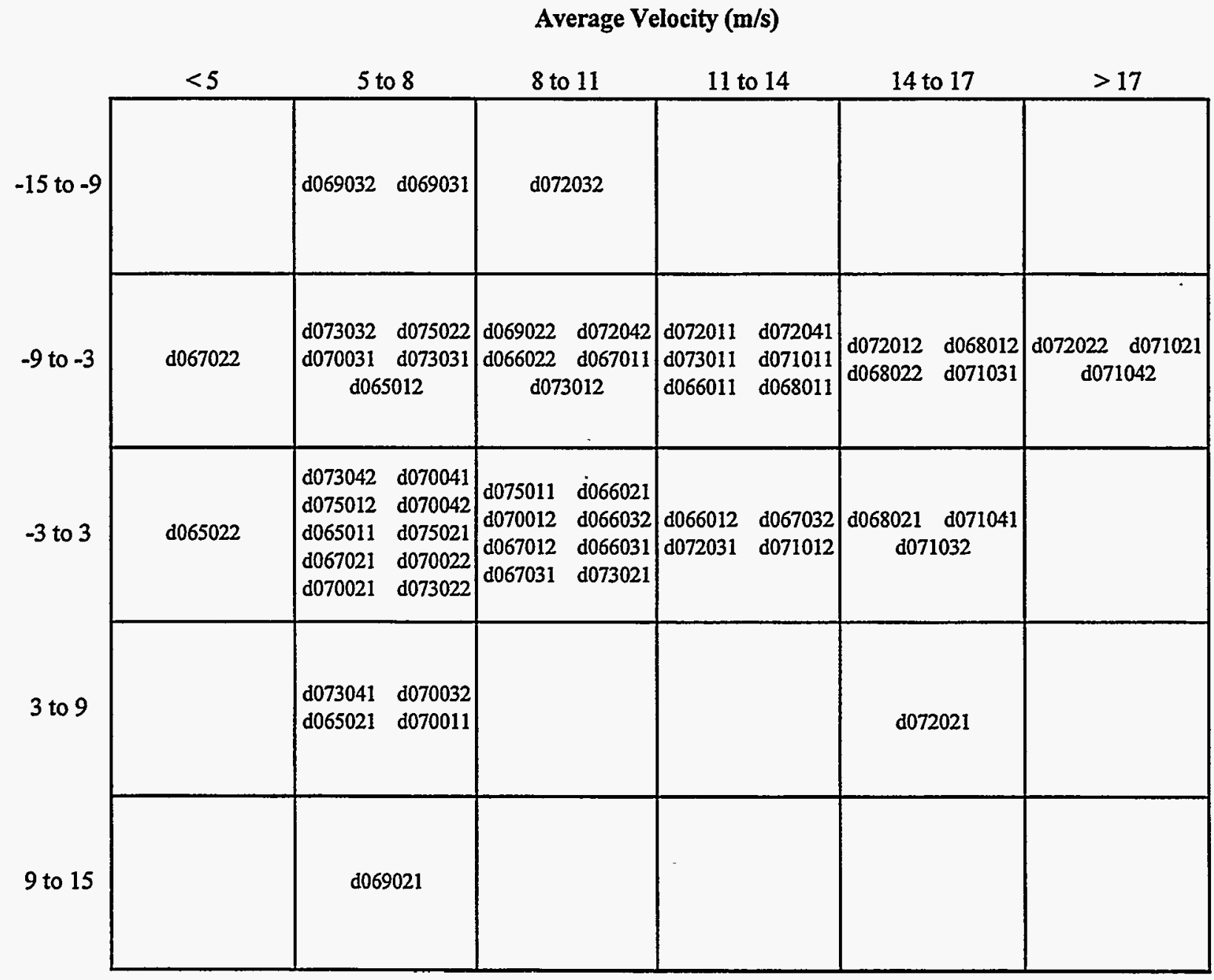

FIGURE 3. DISTRIBUTION OF FIVE-MINUTE DATA EPISODES BASED UPON AVERAGE INFLOW CONDITIONS, YAW, AND VELOCITY. 


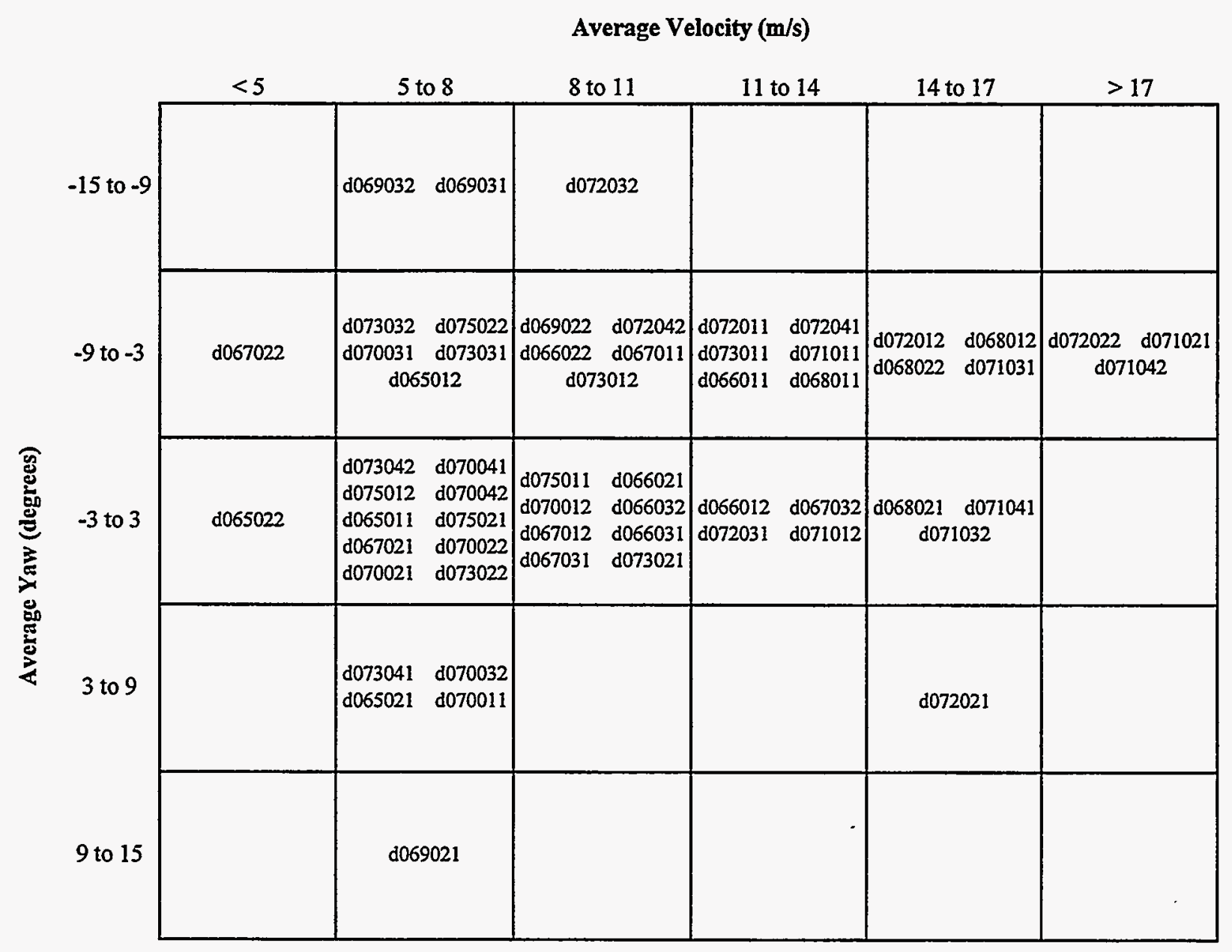

FIGURE 3. DISTRIBUTION OF FIVE-MINUTE DATA EPISODES BASED UPON AVERAGE INFLOW CONDITIONS, YAW, AND VELOCITY. 


\section{Mean Velocity and Standard Deviation}

\begin{tabular}{|c|c|c|c|c|c|c|c|}
\hline & & & verage $\mathrm{Ve}$ & $y(\mathrm{~m} / \mathrm{s})$ & & & \\
\hline & $<5$ & 5 to 8 & 8 to 11 & 11 to 14 & 14 to 17 & $>17$ & Row Avg \\
\hline-15 to -9 & - & $7.4 \pm 1.7$ & $10.3 \pm 3.0$ & - & - & - & $8.3 \pm 2.5$ \\
\hline-9 to -3 & $4.2 \pm 1.4$ & $6.4 \pm 1.5$ & $9.2 \pm 1.9$ & $12.8 \pm 2.9$ & $14.8 \pm 2.8$ & $17.8 \pm 3.2$ & $11.4 \pm 4.7$ \\
\hline-3 to 3 & $4.7 \pm 1.5$ & $7.3 \pm 1.3$ & $9.3 \pm 1.6$ & $12.4 \pm 2.6$ & $15.5 \pm 2.8$ & - & $9.5 \pm 3.6$ \\
\hline 3 to 9 & - & $6.8 \pm 1.5$ & - & - & $14.2 \pm 3.7$ & - & $8.3 \pm 3.6$ \\
\hline 9 to 15 & - & $7.7 \pm 1.4$ & - & - & - & - & $7.7 \pm 1.4$ \\
\hline & $4.6 \cap 1.5$ & $7.0 \pm 1.5$ & $9.3 \pm 1.8$ & $12.6 \pm 2.8$ & $15.0 \pm 2.9$ & $17.8 \pm 3.2$ & $10.1 \pm 4.2$ \\
\hline
\end{tabular}

Mean Yaw and Standard Deviation

\begin{tabular}{|c|c|c|c|c|c|c|c|}
\hline & \multicolumn{5}{|c|}{ Average Velocity $(\mathrm{m} / \mathrm{s})$} & \multirow[b]{2}{*}{$>17$} & \multirow[b]{2}{*}{ Row Avg } \\
\hline & $<5$ & 5 to 8 & 8 to 11 & 11 to 14 & 14 to 17 & & \\
\hline-15 to -9 & - & $-15.4 \pm 15.3$ & $-9.4 \pm 21.3$ & - & - & - & $-13.5 \neq 17.6$ \\
\hline-9 to -3 & $-8.2 \pm 20.2$ & $-5.3 \pm 14.4$ & $-4.6 \pm 11.8$ & $-4.6 \pm 10.9$ & $-4.2 \pm 11.6$ & $-3.6 \pm 12.7$ & $-4.7 \pm 12.7$ \\
\hline-3 to 3 & $-0.3 \pm 9.7$ & $0.0 \pm 11.3$ & $-1.3 \pm 11.6$ & $-1.1 \neq 12.9$ & $-2.1 \pm 11.9$ & - & $-0.9 \pm 11.6$ \\
\hline 3 to 9 & - & $3.8 \pm 12.6$ & - & - & $5.6 \pm 13.7$ & - & $4.2=12.8$ \\
\hline 9 to 15 & - & $13.5 \pm 20.9$ & - & - & - & - & $13.5 \pm 20.9$ \\
\hline & $-2.9 \pm 14.6$ & $-1.4 \pm 14.6$ & $-3.0 \pm 12.7$ & $-3.2 \pm 11.8$ & $-2.2 \pm 12.4$ & $-3.6 \pm 12.7$ & $-2.4 \pm 13.3$ \\
\hline
\end{tabular}

FIGURE 4. DISTRIBUTION OF INFLOW DATA FOR ALL FIVE-MINUTE TEST EPISODES. MEANS AND STANDARD DEVIATIONS ARE GIVEN FOR FREESTREAM VELOCITY IN THE TOP CHART AND YAW IN THE BOTTOM CHART. 

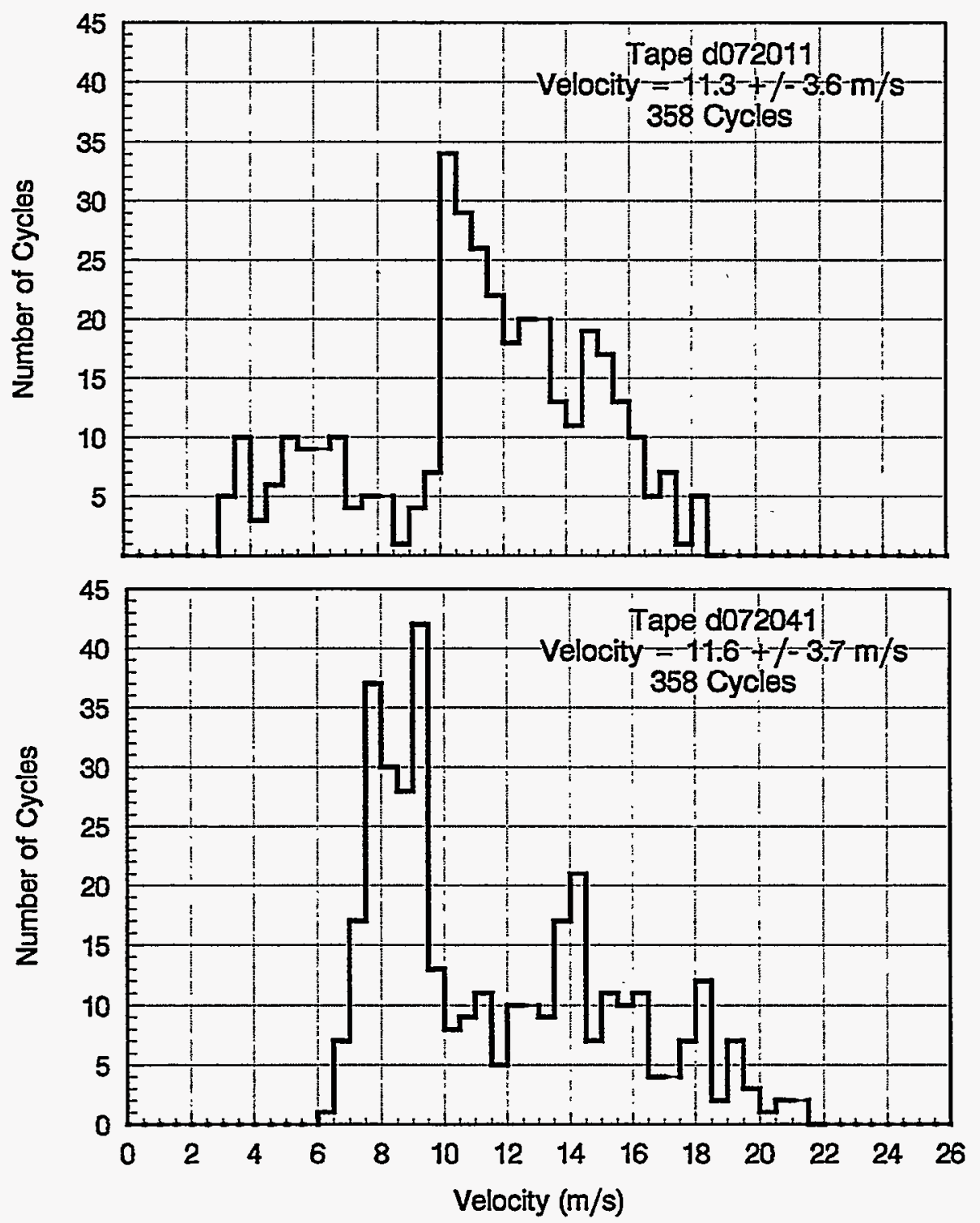

FIGURE 5. VELOCITY DISTRIBUTION HISTOGRAMS FOR SINGLE CYCLES OVER TWO SEPARATE FIVE-MINUTE DATA EPISODES WITH SIMILAR MEAN VELOCITIES. 
TABLE 1. EXAMPLE OF INFLOW CONDITIONS DATABASE *.SUM FILE USING d075022.SUM. THESE FILES CONTAIN THE CYCLE NUMBER ALONG WITH THE CYCLE MEAN AND STANDARD DEVIATION VALUES FOR VELOCITY, YAW, HORIZONTAL SHEAR, AND VERTICAL SHEAR WITH APPROPRIATE TITLES.

\begin{tabular}{|c|c|c|c|c|}
\hline Cyc 1 & Vel: $6.392+-0.079$ & Yaw: $2.079+-2.916$ & HzShr: $0.113 \div-0.011$ & VShr: $0.057+-0.009$ \\
\hline Cyc 2 & Vel: $6.592+-0.042$ & Yaw: $0.763+-1.662$ & HzShr: $0.002+-0.052$ & VShr: $0.081+-0.013$ \\
\hline Cyc 3 & Vel: $6.449+-0.067$ & Yaw: $-6.355+-4.692$ & HzShr: $-0.177+-0.025$ & VShr: $0.066+-0.007$ \\
\hline Cyc 4 & Vel: $6.253+-0.034$ & Yaw: $-5.536+-2.292$ & HzShr: $-0.226+-0.024$ & VShr: $0.014+-0.031$ \\
\hline Cyc 5 & Vel: $6.248+-0.013$ & Yaw: $-5.957+-2.507$ & HzShr: $-0.206+-0.033$ & VShr: $-0.034+-0.007$ \\
\hline Cyc 6 & Vel: $6.345+-0.048$ & Yaw: $-11.910+-4.353$ & HzShr: $-0.056 \div-0.044$ & VShr: $-0.073+-0.016$ \\
\hline Cyc 7 & Vel: $6.421+-0.022$ & Yaw: $-22.322+-0.821$ & HzShr: $-0.011+-0.015$ & VShr: $-0.065+-0.022$ \\
\hline Cyc 8 & Vel: $6.454+-0.021$ & Yaw: $-21.511+-2.636$ & HzShr: $0.029+-0.010$ & VShr: $0.043+-0.022$ \\
\hline Cyc 9 & Vel: $6.386+-0.023$ & Yaw: $-26.589+-2.396$ & HzShr: $0.003+-0.020$ & VShr: $0.090+-0.006$ \\
\hline Cyc 10 & Vel: $6.282+-0.044$ & Yaw: $-23.241+-2.151$ & HzShr: $-0.033+-0.012$ & VShr: $0.127+-0.024$ \\
\hline Cyc 11 & Vel: $6.285+-0.015$ & Yaw: $-26.714+-2.447$ & HzShr: $-0.005+-0.006$ & VShr: $0.111+-0.018$ \\
\hline Cyc 12 & Vel: $6.313+-0.016$ & Yaw: $-27.362+-1.079$ & HzShr: $-0.009+-0.022$ & VShr: $0.032+-0.017$ \\
\hline Cyc 13 & Vel: $6.240+-0.059$ & Yaw: $-21.448+-0.814$ & HzShr: $-0.080+-0.008$ & VShr: $0.054+-0.012$ \\
\hline Cyc 14 & Vel: $6.023+-0.052$ & Yaw: $-21.377+-1.503$ & HzShr: $-0.036 \div-0.019$ & VShr: $0.079+-0.006$ \\
\hline Cyc 15 & Vel: $6.094+-0.075$ & Yaw: $-17.013+-2.115$ & HzShr: $-0.023+-0.009$ & VShr: $0.090+-0.018$ \\
\hline Cyc 16 & Vel: $6.332+-0.047$ & Yaw: $-16.108+-2.447$ & HzShr: $-0.057+-0.012$ & VShr: $0.085+-0.012$ \\
\hline Cyc 17 & Vel: $6.486+-0.047$ & Yaw: $-17.043+-1.484$ & HzShr: $-0.097+-0.004$ & VShr: $0.066+-0.023$ \\
\hline Cyc 18 & Vel: $6.510+-0.038$ & Yaw: $-18.867+-1.409$ & HzShr: $-0.096+-0.012$ & VShr: $-0.013+-0.010$ \\
\hline Cyc 19 & Vel: $6.382+-0.036$ & Yaw: $-20.165+-1.478$ & HzShr: $-0.095+-0.019$ & VShr: $-0.035+-0.011$ \\
\hline Сус 20 & Vel: $6.290+-0.041$ & Yaw: $-19.863+-0.857$ & HzShr: $-0.053+-0.008$ & VShr: $-0.006+-0.021$ \\
\hline
\end{tabular}

TABLE 2. EXAMPLE OF INFLOW CONDITIONS DATABASE *.OUT FILE USING d075022.OUT. THESE FILES CONTAIN THE CYCLE NUMBER ALONG WITH THE CYCLE MEAN AND STANDARD DEVIATION VALUES FOR VELOCITY, YAW, HORIZONTAL SHEAR, AND VERTICAL SHEAR WITHOUT APPROPRIATE TITLES.

\begin{tabular}{|l|l|l|l|l|l|l|l|l|}
\hline 2 & 6.592 & 0.042 & 0.763 & 1.662 & 0.002 & 0.052 & 0.081 & 0.013 \\
\hline 3 & 6.449 & 0.067 & -6.355 & 4.692 & -0.177 & 0.025 & 0.066 & 0.007 \\
\hline 4 & 6.253 & 0.034 & -5.536 & 2.292 & -0.226 & 0.024 & 0.014 & 0.031 \\
\hline 5 & 6.248 & 0.013 & -5.957 & 2.507 & -0.206 & 0.033 & -0.034 & 0.007 \\
\hline 6 & 6.345 & 0.048 & -11.910 & 4.353 & -0.056 & 0.044 & -0.073 & 0.016 \\
\hline 7 & 6.421 & 0.022 & -22.322 & 0.821 & -0.011 & 0.015 & -0.065 & 0.022 \\
\hline 8 & 6.454 & 0.021 & -21.511 & 2.636 & 0.029 & 0.010 & 0.043 & 0.022 \\
\hline 9 & 6.386 & 0.023 & -26.589 & 2.396 & 0.003 & 0.020 & 0.090 & 0.006 \\
\hline 10 & 6.282 & 0.044 & -23.241 & 2.151 & -0.033 & 0.012 & 0.127 & 0.024 \\
\hline 11 & 6.285 & 0.015 & -26.714 & 2.447 & -0.005 & 0.006 & 0.111 & 0.018 \\
\hline 12 & 6.313 & 0.016 & -27.362 & 1.079 & -0.009 & 0.022 & 0.032 & 0.017 \\
\hline 13 & 6.240 & 0.059 & -21.448 & 0.814 & -0.080 & 0.008 & 0.054 & 0.012 \\
\hline 14 & 6.023 & 0.052 & -21.377 & 1.503 & -0.036 & 0.019 & 0.079 & 0.006 \\
\hline 15 & 6.094 & 0.075 & -17.013 & 2.115 & -0.023 & 0.009 & 0.090 & 0.018 \\
\hline 16 & 6.332 & 0.047 & -16.108 & 2.447 & -0.057 & 0.012 & 0.085 & 0.012 \\
\hline 17 & 6.486 & 0.047 & -17.043 & 1.484 & -0.097 & 0.004 & 0.066 & 0.023 \\
\hline 18 & 6.510 & 0.038 & -18.867 & 1.409 & -0.096 & 0.012 & -0.013 & 0.010 \\
\hline 19 & 6.382 & 0.036 & -20.165 & 1.478 & -0.095 & 0.019 & -0.035 & 0.011 \\
\hline 20 & 6.290 & 0.041 & -19.863 & 0.857 & -0.053 & 0.008 & -0.006 & 0.021 \\
\hline
\end{tabular}




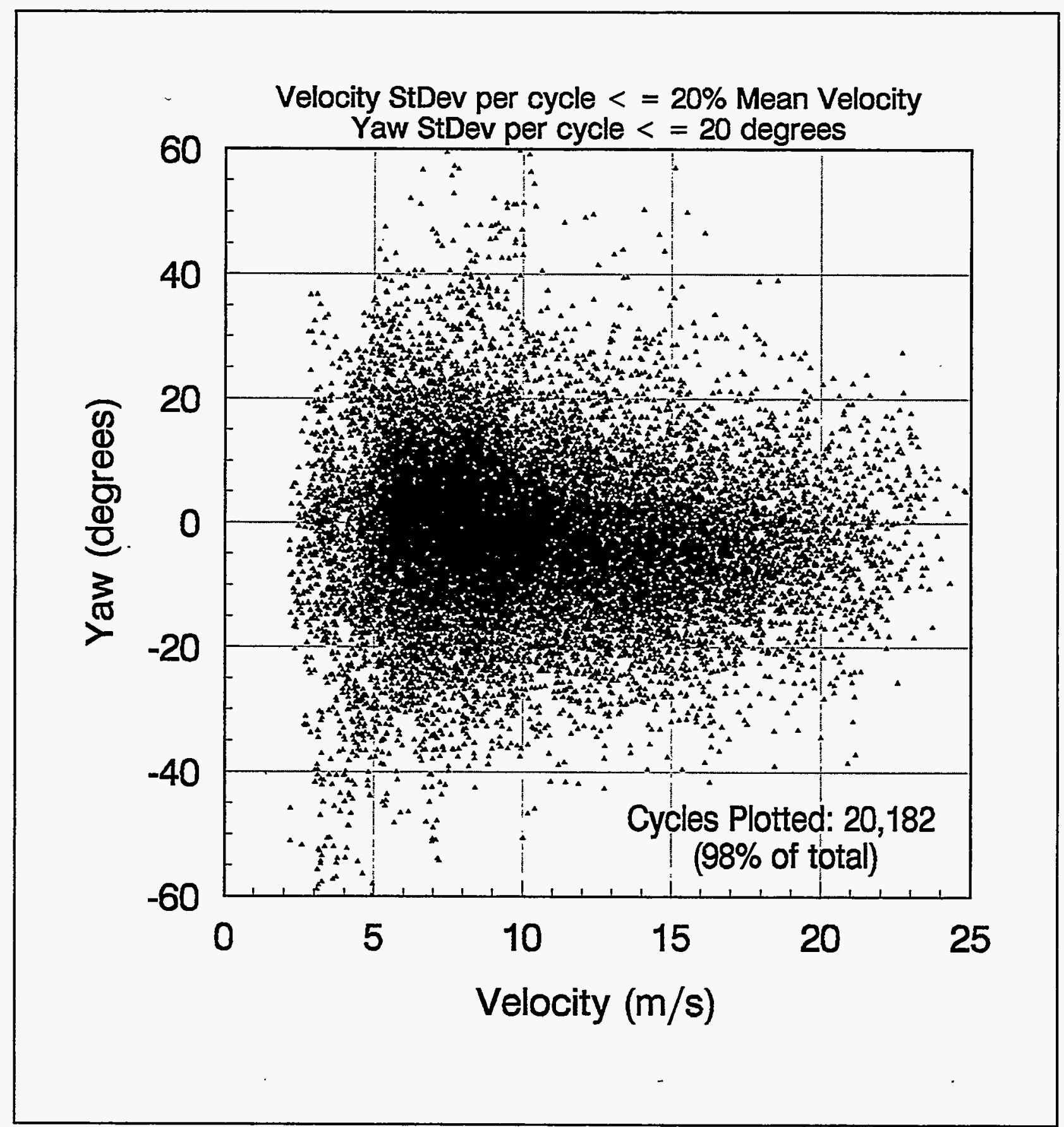

FIGURE 6. DISTRIBUTION OF AVERAGE INFLOW CONDITIONS FOR EACH INDIVIDUAL CYCLE. THE DATA FROM ALL 59 DATA EPISODES IS PLOTTED. 
TABLE 3. EXAMPLE OF AERODYNAMIC FORCE COEFFICIENTS DATABASE *CN.SUM FILE USING d075021CN.SUM. THESE FILES CONTAIN THE CYCLE NUMBER ALONG WITH THE CYCLE MEAN AND STANDARD DEVIATION VALUES FOR THE NORMAL COEFFICIENT AT $30 \%, 47 \%, 63 \%$, AND $80 \%$ SPAN WITH APPROPRIATE TITLES.

\begin{tabular}{|c|c|c|c|c|}
\hline cyc: 1 & $30 \%: 1.724+-0.357$ & $47 \%: 0.939+-0.134$ & $63 \%: 0.670+-0.090$ & $80 \%: 0.340+-0.092$ \\
\hline cyc: 2 & $30 \%: 1.490+-0.238$ & $47 \%: 0.898+-0.125$ & $63 \%: 0.617+-0.087$ & $80 \%: 0.314+-0.085$ \\
\hline cyc: 3 & $30 \%: 1.115+-0.166$ & $47 \%: 0.778+-0.110$ & $63 \%: 0.551+-0.114$ & $80 \%: 0.281+-0.091$ \\
\hline cyc: 4 & $30 \%: 1.308+-0.209$ & $47 \%: 0.846+-0.146$ & $63 \%: 0.560+-0.118$ & $80 \%: 0.281+-0.107$ \\
\hline cyc: 5 & $30 \%: 1.446+-0.194$ & $47 \%: 0.890+-0.115$ & $63 \%: 0.708+-0.101$ & $80 \%: 0.409+-0.077$ \\
\hline cyc: 6 & $30 \%: 1.508+-0.201$ & $47 \%: 0.932+-0.123$ & $63 \%: 0.770+-0.102$ & $80 \%: 0.455+-0.087$ \\
\hline cyc: 7 & $30 \%: 1.455+-0.283$ & $47 \%: 0.903+-0.161$ & $63 \%: 0.713+-0.088$ & $80 \%: 0.421+-0.087$ \\
\hline cyc: 8 & $30 \%: 1.539+-0.250$ & $47 \%: 0.895+-0.088$ & $63 \%: 0.688+-0.093$ & $80 \%: 0.374+-0.084$ \\
\hline cyc: 9 & $30 \%: 1.425+-0.184$ & $47 \%: 0.843+-0.100$ & $63 \%: 0.638+-0.079$ & $80 \%: 0.348+-0.075$ \\
\hline cyc: 10 & $30 \%: 1.330+-0.140$ & $47 \%: 0.841+-0.111$ & $63 \%: 0.659+-0.109$ & $80 \%: 0.379+-0.112$ \\
\hline cyc: 11 & $30 \%: 1.488+-0.301$ & $47 \%: 0.884+-0.114$ & $63 \%: 0.689+-0.123$ & $80 \%: 0.413+-0.106$ \\
\hline cyc: 12 & $30 \%: 1.463+-0.192$ & $47 \%: 0.882+-0.122$ & $63 \%: 0.685+-0.103$ & $80 \%: 0.377+-0.116$ \\
\hline cyc: 13 & $30 \%: 1.233+-0.112$ & $47 \%: 0.819+-0.109$ & $63 \%: 0.598+-0.091$ & $80 \%: 0.339+-0.079$ \\
\hline cyc: 14 & $30 \%: 1.173+-0.226$ & $47 \%: 0.785+-0.104$ & $63 \%: 0.532+-0.076$ & $80 \%: 0.266+-0.083$ \\
\hline cyc: 15 & $30 \%: 1.471+-0.227$ & $47 \%: 0.878+-0.180$ & $63 \%: 0.655+-0.112$ & $80 \%: 0.342+-0.093$ \\
\hline cyc: 16 & $30 \%: 1.429+-0.236$ & $47 \%: 0.891+-0.114$ & $63 \%: 0.702+-0.105$ & $80 \%: 0.399+-0.095$ \\
\hline cyc: 17 & $30 \%: 1.401+-0.255$ & $47 \%: 0.905+-0.116$ & $63 \%: 0.701+-0.112$ & $80 \%: 0.400+-0.115$ \\
\hline cyc: 18 & $30 \%: 1.474+-0.230$ & $47 \%: 0.919+-0.111$ & $63 \%: 0.725+-0.170$ & $80 \%: 0.412+-0.146$ \\
\hline cyc: 19 & $30 \%: 1.479+-0.228$ & $47 \%: 0.895+-0.134$ & $63 \%: 0.670+-0.161$ & $80 \%: 0.388+-0.151$ \\
\hline cyc: 20 & $30 \%: 1.621+-0.323$ & $47 \%: 0.937+-0.174$ & $63 \%: 0.761+-0.082$ & $80 \%: 0.456+-0.099$ \\
\hline
\end{tabular}

TABLE 4. EXAMPLE OF AERODYNAMIC FORCE COEFFICIENTS DATABASE *CT.SUM FILE USING d075021CT.SUM. THESE FILES CONTAIN THE CYCLE NUMBER ALONG WITH THE CYCLE MEAN AND STANDARD DEVIATION VALUES FOR THE NORMAL COEFFICIENT AT $30 \%, 47 \%, 63 \%$, AND $80 \%$ SPAN WITH APPROPRIATE TITLES.

\begin{tabular}{|c|c|c|c|c|}
\hline cyc: 1 & $30 \%: 0.096+-0.075$ & $47 \%: 0.135+-0.029$ & $63 \%: 0.052+-0.015$ & $80 \%: 0.001+-0.005$ \\
\hline cyc: 2 & $30 \%: 0.084+-0.073$ & $47 \%: 0.121+-0.024$ & $63 \%: 0.040+-0.014$ & $80 \%:-0.002+-0.006$ \\
\hline cyc: 3 & $30 \%: 0.121+-0.036$ & $47 \%: 0.099+-0.020$ & $63 \%: 0.035+-0.019$ & $80 \%:-0.002+-0.007$ \\
\hline cyc: 4 & $30 \%: 0.119+-0.056$ & $47 \%: 0.110+-0.034$ & $63 \%: 0.035+-0.017$ & $80 \%:-0.002+-0.007$ \\
\hline cyc: 5 & $30 \%: 0.070+-0.056$ & $47 \%: 0.133+-0.026$ & $63 \%: 0.064+-0.020$ & $80 \%: 0.009+-0.008$ \\
\hline cyc: 6 & $30 \%: 0.065+-0.073$ & $47 \%: 0.143+-0.028$ & $63 \%: 0.079+-0.020$ & $80 \%: 0.013+-0.010$ \\
\hline cyc: 7 & $30 \%: 0.032+-0.078$ & $47 \%: 0.138+-0.033$ & $63 \%: 0.064+-0.018$ & $80 \%: 0.009+-0.008$ \\
\hline cyc: 8 & $30 \%: 0.021+-0.077$ & $47 \%: 0.134+-0.020$ & $63 \%: 0.060+-0.016$ & $80 \%: 0.004+-0.007$ \\
\hline cyc: 9 & $30 \%: 0.050+-0.064$ & $47 \%: 0.122+-0.025$ & $63 \%: 0.050+-0.015$ & $80 \%: 0.003+-0.007$ \\
\hline cyc: 10 & $30 \%: 0.102+-0.049$ & $47 \%: 0.122+-0.025$ & $63 \%: 0.053+-0.019$ & $80 \%: 0.005+-0.009$ \\
\hline cyc: 11 & $30 \%: 0.079+-0.066$ & $47 \%: 0.132+-0.030$ & $63 \%: 0.057+-0.026$ & $80 \%: 0.010+-0.011$ \\
\hline cyc: 12 & $30 \%: 0.054+-0.072$ & $47 \%: 0.126+-0.027$ & $63 \%: 0.058+-0.020$ & $80 \%: 0.007+-0.011$ \\
\hline cyc: 13 & $30 \%: 0.100+-0.047$ & $47 \%: 0.106+-0.023$ & $63 \%: 0.046+-0.019$ & $80 \%: 0.003+-0.006$ \\
\hline cyc: 14 & $30 \%: 0.096+-0.054$ & $47 \%: 0.097+-0.029$ & $63 \%: 0.031+-0.013$ & $80 \%:-0.003+-0.006$ \\
\hline cyc: 15 & $30 \%: 0.053+-0.075$ & $47 \%: 0.121+-0.042$ & $63 \%: 0.052+-0.022$ & $80 \%: 0.003+-0.008$ \\
\hline cyc: 16 & $30 \%: 0.032+-0.075$ & $47 \%: 0.133+-0.027$ & $63 \%: 0.063+-0.021$ & $80 \%: 0.008+-0.009$ \\
\hline cyc: 17 & $30 \%: 0.046+-0.066$ & $47 \%: 0.134+-0.028$ & $63 \%: 0.064+-0.024$ & $80 \%: 0.009+-0.012$ \\
\hline cyc: 18 & $30 \%: 0.046+-0.069$ & $47 \%: 0.133+-0.031$ & $63 \%: 0.072+-0.034$ & $80 \%: 0.013+-0.015$ \\
\hline cyc: 19 & $30 \%: 0.021+-0.082$ & $47 \%: 0.109+-0.042$ & $63 \%: 0.061+-0.030$ & $80 \%: 0.011+-0.015$ \\
\hline cyc: 20 & $30 \%: 0.008+-0.090$ & $47 \%: 0.102+-0.068$ & $63 \%: 0.080+-0.022$ & $80 \%: 0.017+-0.015$ \\
\hline
\end{tabular}


TABLE 5. EXAMPLE OF AERODYNAMIC FORCE COEFFICIENT DATABASE *MAX.SUM FILE USING d075021MAX.SUM. THESE FILES CONTAIN THE CYCLE NUMBER ALONG WITH THE CYCLE'S MAXIMUM NORMAL AND TANGENT COEFFICIENT AT 30\%, 47\%, 63\%, AND $80 \%$ SPAN WITH APPROPRIATE TITLES.

\begin{tabular}{|c|c|c|c|c|c|c|c|c|}
\hline cyc: 1 & $\operatorname{cn} 30 \%: 2.279$ & $\operatorname{cn} 47 \%: 1.136$ & cn63\%: 0.865 & $\operatorname{cn} 80 \%: 0.500$ & ct30\%: 0.258 & ct $47 \%: 0.183$ & ct63\%: 0.074 & ct80\%: 0.014 \\
\hline cyc: 2 & $\operatorname{cn} 30 \%: 2.048$ & cn47\%: 1.153 & cn63\%: 0.873 & cn80\%: 0.535 & ct30\%: 0.251 & ct $47 \%: 0.160$ & ct63\%: 0.081 & ct80\%: 0.016 \\
\hline cyc: 3 & cn30\%: 1.381 & cn47\%: 1.030 & n63\%: 0.942 & cn80\%: 0.543 & ct30\%: 0.205 & ct47\%: 0.144 & ct63\%: 0.102 & ct80\%: 0.025 \\
\hline cyc: 4 & cn30\%: 1.705 & cn47\%: 1.164 & cn63\%: 0.857 & cn80\%: 0.542 & ct30\%: 0.244 & ct $47 \%: 0.196$ & ct63\%: 0.076 & ct80\%: 0.022 \\
\hline cyc: 5 & $\operatorname{cn} 30 \%: 1.820$ & cn47\%: 1.096 & cn63\%: 0.926 & cn80\%: 0.565 & ct30\%: 0.220 & ct $47 \%: 0.187$ & ct63\%: 0.108 & ct80\%: 0.031 \\
\hline cyc: 6 & cn30\%: 1.911 & cn47\%: 1.127 & cn63\%: 1.005 & cn80\%: 0.578 & ct30\%: 0.244 & ct $47 \%: 0.195$ & ct63\%: 0.122 & ct80\%: 0.036 \\
\hline cyc: 7 & cn30\%: 1.773 & $\operatorname{cn} 47 \%: 1.119$ & cn63\%: 0.930 & cn80\%: 0.613 & ct30\%: 0.277 & ct $47 \%: 0.192$ & ct63\%: 0.111 & ct $80 \%: 0.035$ \\
\hline cyc: 8 & cn30\%: 1.977 & cn $47 \%: 1.131$ & cn63\%: 0.917 & cn80\%: 0.563 & ct30\%: 0.242 & ct $47 \%: 0.193$ & ct63\%: 0.101 & ct $80 \%: 0.022$ \\
\hline cyc: 9 & $\operatorname{cn} 30 \%: 1.841$ & cn $47 \%: 0.998$ & cn63\%: 0.829 & $\operatorname{cn} 80 \%: 0.555$ & ct30\%: 0.209 & ct $47 \%: 0.175$ & ct63\%: 0.087 & ct $80 \%: 0.023$ \\
\hline cyc: 10 & $\operatorname{cn} 30 \%: 1.786$ & cn47\%: 1.077 & cn63\%: 0.883 & cn80\%: 0.589 & ct30\%: 0.203 & ct $47 \%: 0.178$ & ct63\%: 0.099 & ct $80 \%: 0.030$ \\
\hline cyc: 11 & $\operatorname{cn} 30 \%: 2.201$ & cn47\%: 1.213 & cn63\%: 0.959 & cn80\%: 0.611 & ct30\%: 0.242 & ct $47 \%: 0.227$ & ct63\%: 0.117 & ct80\%: 0.037 \\
\hline cyc: 12 & $\operatorname{cn} 30 \%: 2.246$ & cn47\%: 1.153 & cn63\%: 1.017 & cn80\%: 0.616 & ct30\%: 0.211 & ct $47 \%: 0.191$ & ct63\%: 0.118 & ct80\%: 0.041 \\
\hline cyc: 13 & cn30\%: 1.568 & cn $47 \%: 1.079$ & cn63\%: 0.815 & cn $80 \%: 0.480$ & ct30\%: 0.192 & ct $47 \%: 0.164$ & ct63\%: 0.095 & ct80\%: 0.021 \\
\hline cyc: 14 & cn30\%: 1.654 & cn47\%: 1.083 & cn63\%: 0.706 & cn $80 \%: 0.390$ & ct30\%: 0.210 & ct $47 \%: 0.170$ & ct63\%: 0.072 & ct80\%: 0.012 \\
\hline cyc: 15 & $\operatorname{cn} 30 \%: 1.800$ & cn47\%: 1.268 & cn63\%: 0.894 & cn80\%: 0.503 & ct30\%: 0.262 & ct $47 \%: 0.196$ & ct63\%: 0.094 & ct80\%: 0.021 \\
\hline
\end{tabular}

TABLE 6. EXAMPLE OF AERODYNAMIC FORCE COEFFICIENT DATABASE *.ERR FILE USING d075021.ERR. THESE FILES CONTAIN THE ANOMALOUS AND THE CORRECTED POINTS ALONG WITH THE CYCLE NUMBER AND THE SPAN LOCATION OF THE ERROR.

Anomalous points listing from $\mathrm{d} 075021$

change cn 1.293289 to 0.680574

change ct -0.059162 to 0.068494

in cyc 4 , at $63 \%$ span

change $\mathrm{cn}-1.541332$ to 0.769346

change ct 0.556902 to 0.074663

change cn 12.129753 to 0.771591

change ct -0.175177 to 0.076658

in cyc 11 , at $63 \%$ span 
TABLE 7. EXAMPLE OF CNCTMAX.F OUTPUT FILE BASED ON THE TANGENT FORCE COEFFICIENT AT 47\% SPAN. THIS FILE CONTAINS RANK, TAPE NAME, CYCLE NUMBER, THE CYCLE'S MEAN ANGLE OF ATTACK, MAXIMUM TANGENT FORCE FOR THE CYCLE, AS WELL AS THE MEAN AND STANDARD DEVIATION VALUES FOR TANGENT FORCE, VELOCITY, AND YAW.

\begin{tabular}{|c|c|c|c|c|c|c|c|c|c|c|}
\hline Rank & Tape & Cyc & Mean Ct & Ct sd & Max Ct & AOA & Mean Vel & Vsd & Mean Yaw & Ysd \\
\hline 1 & $\mathrm{~d} 071032$ & 41 & 0.898 & 0.704 & 1.48 & 30.518 & 17.590 & 0.453 & -15.778 & 3.698 \\
\hline 2 & $\mathrm{~d} 073032$ & 107 & 0.407 & 0.074 & 0.89 & 2.194 & 5.319 & 0.078 & -4.860 & 1.970 \\
\hline 3 & $\mathrm{~d} 073022$ & 143 & 0.164 & 0.033 & 0.22 & 8.648 & 7.829 & 0.069 & 6.595 & 5.317 \\
\hline 4 & $\mathrm{~d} 072042$ & 155 & 0.160 & 0.055 & 0.27 & 9.406 & 8.129 & 0.095 & -7.337 & 1.650 \\
\hline 5 & $\mathrm{~d} 073021$ & 312 & 0.160 & 0.026 & 0.21 & 12.166 & 9.063 & 0.068 & -2.150 & 0.331 \\
\hline 6 & $\mathrm{~d} 072032$ & 267 & 0.159 & 0.078 & 0.32 & 8.706 & 8.233 & 0.282 & 22.100 & 9.522 \\
\hline 7 & $\mathrm{~d} 072022$ & 273 & 0.155 & 0.122 & 0.34 & 19.954 & 14.208 & 0.077 & -39.543 & 5.686 \\
\hline 8 & $\mathrm{~d} 073012$ & 66 & 0.155 & 0.045 & 0.27 & 9.962 & 8.273 & 0.314 & 3.241 & 2.502 \\
\hline 9 & $\mathrm{~d} 073022$ & 248 & 0.154 & 0.024 & 0.20 & 10.807 & 8.615 & 0.101 & 7.973 & 1.055 \\
\hline 10 & $\mathrm{~d} 072042$ & 210 & 0.154 & 0.040 & 0.22 & 12.196 & 9.085 & 0.109 & -3.716 & 2.394 \\
\hline 11 & $\mathrm{~d} 072042$ & 258 & 0.154 & 0.037 & 0.25 & 14.025 & 9.774 & 0.061 & -5.761 & 1.122 \\
\hline 12 & $\mathrm{~d} 073022$ & 247 & 0.151 & 0.035 & 0.20 & 11.654 & 8.908 & 0.037 & 7.215 & 1.339 \\
\hline 13 & $\mathrm{~d} 071011$ & 335 & 0.151 & 0.044 & 0.24 & 10.535 & 8.492 & 0.099 & 5.558 & 1.247 \\
\hline 14 & $\mathrm{~d} 073022$ & 211 & 0.150 & 0.033 & 0.21 & 11.293 & 8.816 & 0.044 & 9.813 & 1.760 \\
\hline 15 & $\mathrm{~d} 072021$ & 330 & 0.150 & 0.024 & 0.19 & 10.301 & 8.411 & 0.025 & 5.813 & 0.657 \\
\hline
\end{tabular}

TABLE 8. EXAMPLE OF MINIMUM LEADING-EDGE PRESSURE COEFFICIENTS DATABASE USING MAXCP65011R.DAT. THESE FILES CONTAIN THE TAPE AND CYCLE NUMBER ALONG WITH THE MINIMUM LEADING-EDGE PRESSURE COEFFICIENT FOR EACH SPAN LOCATION AND THE INSTANTANEOUS VELOCITY, YAW, AND AZIMUTH VALUES AT WHICH THE PRESSURE COEFFICIENT OCCURRED. THE HEADER FOR THE FILE IS ALSO INCLUDED.

The maximum leading edge suction in each cycle is listed below

The Cp's were re-normalized using a yaw dependent dynamic pressure

The entire cycle was evaluated for max leading edge suction

All wind velocities were evaluated for max leading edge suction

All yaws were evaluated for max leading edge suction

\begin{tabular}{|c|c|c|c|c|c|c|}
\hline Tape & Cycle & Span & Velocity & Yaw & Max Cp & Azimuth \\
\hline $\mathrm{d} 065011$ & 2 & 30 & 5.832 & 5.453 & -5.5472 & 278.4697 \\
\hline $\mathrm{d} 065011$ & 2 & 47 & 5.843 & 5.133 & -3.4009 & 344.9312 \\
\hline $\mathrm{d} 065011$ & .2 & 63 & 5.832 & 5.368 & -0.9282 & 294.8799 \\
\hline $\mathrm{d} 065011$ & 2 & 80 & 5.832 & 5.453 & 0.3685 & 282.5722 \\
\hline $\mathrm{d} 065011$ & 3 & 30 & 5.834 & 4.860 & -6.0285 & 296.9729 \\
\hline $\mathrm{d} 065011$ & 3 & 47 & 5.843 & 4.963 & -3.1553 & 0.5209 \\
\hline $\mathrm{d} 065011$ & 3 & 63 & 5.843 & 4.963 & -0.7646 & 4.6235 \\
\hline $\mathrm{d} 065011$ & 3 & 80 & 5.840 & 5.559 & 0.4137 & 24.3158 \\
\hline $\mathrm{d} 065011$ & 4 & 30 & 5.823 & -3.720 & -6.4344 & 233.3655 \\
\hline $\mathrm{d} 065011$ & 4 & 47 & 5.787 & -2.232 & -3.5243 & 171.3513 \\
\hline $\mathrm{d} 065011$ & 4 & 63 & 5.831 & -2.314 & -0.5690 & 330.9015 \\
\hline $\mathrm{d} 065011$ & 4 & 80 & 5.810 & -3.369 & 0.3410 & 182.1178 \\
\hline $\mathrm{d} 065011$ & 5 & 30 & 5.817 & 0.395 & -5.7351 & 287.4024 \\
\hline $\mathrm{d} 065011$ & 5 & 47 & 5.778 & 0.927 & -3.1869 & 82.4892 \\
\hline $\mathrm{d} 065011$ & 5 & 63 & 5.778 & 0.927 & -0.6048 & 82.4892 \\
\hline $\mathrm{d} 065011$ & 5 & 80 & 5.814 & 0.203 & 0.5778 & 345.2203 \\
\hline
\end{tabular}



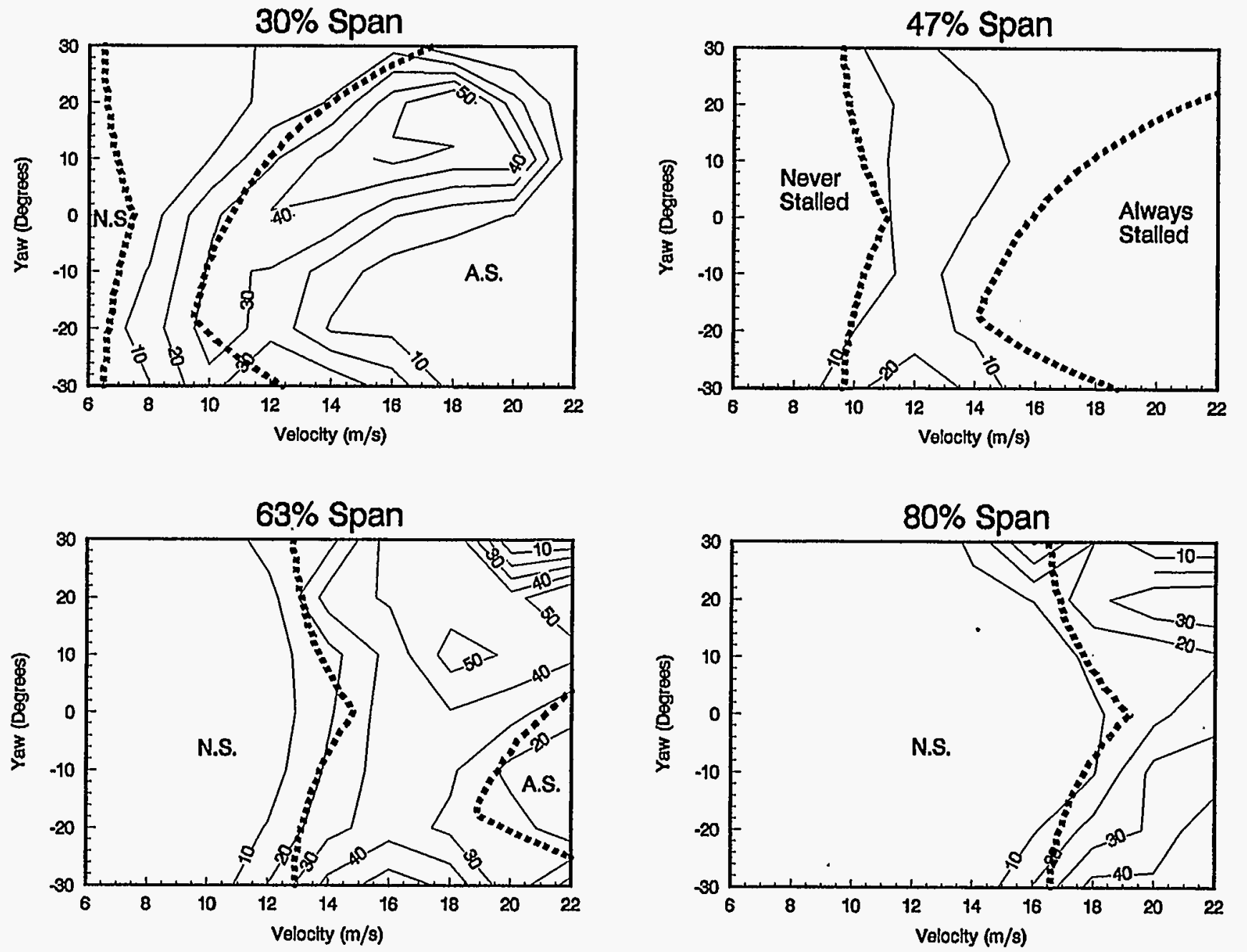

FIGURE 7. FREQUENCY OF DYNAMIC STALL OCCURRENCE AT FOUR SPAN LOCATIONS UNDER VARIOUS INFLOW CONDITIONS OF YAW AND WIND VELOCITY. CONTOURS REPRESENT PERCENTAGE OF CYCLES IN EACH BIN HAVING A PEAK Cp <-10. 


\section{Appendix A}

\section{Single Parameter Tables for Entire Data Episodes}

I. Disc Averaged Wind Speed (m/s), Channel 734

II. Yaw (deg), Channel 307 - Channel 210

III. Measured Dynamic Pressure at $86 \%$ Span (psi), Channel 458

IV. Measured Dynamic Pressure at 67.3\% Span (psi), Channel 934

V. Measured Dynamic Pressure at 50.6\% Span (psi), Channel 862

VI. Measured Dynamic Pressure at 34\% Span (psi), Channel 832

VII. Instrumented Blade Pitch Angle (deg), Channel 461

VIII. Root Flap Bending Moment ( ${ }^{*} \mathrm{~m}$ ), Blade 1 (Instrumented), Channel 402

IX. Root Flap Bending Moment $\left(\mathrm{N}^{*} \mathrm{~m}\right)$, Blade 2, Channel 403

$\mathrm{X}$. Root Flap Bending Moment $\left(\mathrm{N}^{*} \mathrm{~m}\right)$, Blade 3, Channel 404

XI. Root Edge Bending Moment ( $\left.N^{*} \mathrm{~m}\right)$, Channel 414

XII. Low Speed Shaft Torque (N*m), Channel 423

XIII. Generator Power (kW), Channel 308

XIV. Normal Force Coefficient, Cn, at $80 \%$ Span, Channel 714

XV. Normal Force Coefficient, $\mathrm{Cn}$, at $63 \%$ Span, Channel 715

XVI. Normal Force Coefficient, Cn, at $47 \%$ Span, Channel 716

XVII. Normal Force Coefficient, $\mathrm{Cn}$, at 30\% Span, Channel 717

XVIII. Tangent Force Coefficient, $\mathrm{Ct}$, at $80 \%$ Span, Channel 718

XIX. Tangent Force Coefficient, $\mathrm{Ct}$, at $63 \%$ Span, Channel 719

XX. Tangent Force Coefficient, $\mathrm{Ct}$, at $47 \%$ Span, Channel 720

XXI. Tangent Force Coefficient, $\mathrm{Ct}$, at 30\% Span, Channel 721

XXII. Angle of Attack at 82\% Span (deg), Channel 935

XXIII. Angle of Attack at 63.3\% Span (deg), Channel 936

XXIV. Angle of Attack at 46.6\% Span (deg), Channel 937

XXV. Angle of Attack at 30\% Span (deg), Channel 938 
734: Disc Averaged Wind Speed $(\mathrm{m} / \mathrm{s})$

\begin{tabular}{|c|c|c|c|c|c|c|c|c|}
\hline Mean & Std & Min & $\operatorname{Max}$ & Tape & Event & Time Length & Start Time & End Time \\
\hline 4.246 & 1.382 & 2.169 & 8.622 & 67 & 22 & 298.959 & 13516430.791 & 13516929.750 \\
\hline 4.429 & 1.319 & 2.371 & 6.924 & 65 & 22 & 299.370 & 11594740.501 & 11595239.869 \\
\hline 4.960 & 1.704 & 2.199 & 10.630 & 73 & 42 & 299.532 & 153121250.225 & 153121749.755 \\
\hline 5.279 & 1.054 & 2.717 & 7.752 & 73 & 32 & 273.569 & 15312226.020 & 15312659.586 \\
\hline 5.785 & 1.264 & 2.672 & 8.471 & 73 & 41 & 298.510 & 15312750.870 & 153121249.378 \\
\hline 5.865 & 1.104 & 3.752 & 8.930 & 75 & 22 & 289.576 & 20610335.072 & 206103754.646 \\
\hline 6.087 & 1.104 & 3.920 & 8.078 & 70 & 31 & 299.305 & 135214520.167 & 135215019.470 \\
\hline 6.231 & 0.780 & 4.621 & 8.684 & 70 & 41 & 299.230 & 1352157.617 & 13522159.845 \\
\hline 6.300 & 1.540 & 3.989 & 10.270 & 70 & 32 & 299.151 & 135215020.307 & 135215519.458 \\
\hline 6.786 & 1.379 & 3.818 & 10.690 & 75 & 12 & 299.929 & 206101410.124 & 206101910.051 \\
\hline 6.925 & 1.450 & 4.109 & 11.250 & 73 & $\overrightarrow{31}$ & 298.996 & 153115720.314 & 15312219.306 \\
\hline 7.114 & 2.118 & 3.146 & 10.500 & 69 & 32 & 293.222 & 135203546.647 & 135204039.869 \\
\hline 7.242 & 0.951 & 4.866 & 10.140 & 70 & 42 & 293.820 & 1352225.718 & 13522659.537 \\
\hline 7.351 & 1.296 & 4.568 & 9.772 & 65 & 21 & 299.355 & 11594240.316 & 11594739.669 \\
\hline 7.362 & 1.565 & 4.824 & 11.780 & 65 & 11 & 299.439 & 11593115.541 & 11593614.980 \\
\hline 7.377 & 1.116 & 5.124 & 9.983 & 75 & 21 & 299.812 & $2061028 \quad .205$ & $2061033 \quad .013$ \\
\hline 7.423 & 1.223 & 5.191 & 9.900 & 67 & 21 & 299.545 & 135155930.414 & 13516429.957 \\
\hline 7.561 & 1.350 & 5.131 & 10.930 & 70 & 22 & 254.371 & 135213945.583 & 135214359.952 \\
\hline 7.568 & 0.768 & 5.573 & 9.771 & 70 & 21 & 298.798 & 135213440.919 & 135213939.715 \\
\hline 7.583 & 1.055 & 5.188 & 10.370 & 69 & 31 & 299.679 & 135203040.268 & 135203539.945 \\
\hline 7.663 & 1.442 & 4.781 & 10.610 & 69 & 21 & 254.521 & 135201945.149 & 135202359.668 \\
\hline 7.665 & 1.663 & 3.800 & 11.720 & 65 & 12 & 299.224 & 11593615.822 & 11594115.046 \\
\hline 7.680 & 0.969 & 5.483 & 10.070 & 70 & 11 & 299.601 & 135212335.137 & 135212834.737 \\
\hline 7.822 & 1.539 & 5.253 & 12.040 & 73 & 22 & 292.729 & 153115146.543 & 153115639.272 \\
\hline 8.177 & 1.856 & 4.193 & 11.770 & 69 & 22 & 224.076 & 135202445.731 & 135202829.803 \\
\hline 8.204 & 1.126 & 5.687 & 10.810 & 75 & 11 & 299.439 & 20610910.688 & 206101410.124 \\
\hline 8.397 & 1.610 & 4.820 & 12.720 & 72 & 42 & 289.056 & 138171430.447 & 138171919.501 \\
\hline 8.417 & 1.096 & 5.725 & 11.060 & 66 & 21 & 299.234 & 13514240.759 & 13514739.991 \\
\hline 8.436 & 1.107 & 5.341 & 11.030 & 70 & 12 & 299.255 & 135212835.569 & 135213334.824 \\
\hline 8.657 & 1.251 & 5.765 & 11.850 & 66 & 22 & 299.046 & 13514740.831 & 135141239.875 \\
\hline 8.962 & 1.440 & 6.255 & 12.670 & 66 & 32 & 298.883 & 135141910.642 & 13514249.523 \\
\hline 9.009 & 1.132 & 6.505 & 12.440 & 67 & 12 & 298.846 & 135155240.559 & 135155739.403 \\
\hline 9.428 & 1.246 & 6.756 & 13.110 & 67 & 11 & 299.499 & 135154740.226 & 135155239.723 \\
\hline 10.280 & 2.989 & 4.858 & 18.430 & 72 & 32 & 261.823 & 1381746.242 & 13817828.064 \\
\hline 10.320 & 1.431 & 6.213 & 15.070 & 67 & 31 & 299.288 & $1351611 \quad .492$ & 135161559.778 \\
\hline 10.320 & 1.867 & 5.962 & 14.940 & 66 & 31 & 299.449 & 135141410.599 & 135141910.046 \\
\hline 10.620 & 1.202 & 6.866 & 13.910 & 73 & 21 & 299.151 & 153114640.756 & 153115139.904 \\
\hline 10.950 & 1.814 & 7.030 & 14.910 & 73 & 12 & 300.008 & 153114050.094 & 153114550.101 \\
\hline 11.170 & 1.481 & 7.551 & 15.120 & 66 & 12 & 297.491 & 135135345.999 & 135135843.489 \\
\hline 11.320 & 3.595 & 3.187 & 18.790 & 72 & 11 & 299.750 & 138163330.441 & 138163830.191 \\
\hline 11.360 & 1.489 & 8.023 & 15.320 & 67 & 32 & 299.149 & $1351616 \quad .526$ & 135162059.672 \\
\hline 11.600 & 3.738 & 6.329 & 21.840 & 72 & 41 & 298.986 & 13817930.796 & 138171429.779 \\
\hline 13.060 & 2.237 & 7.341 & 18.260 & 73 & 11 & 298.742 & 153113550.765 & 153114049.504 \\
\hline 13.270 & 2.329 & 8.381 & 18.910 & 71 & 11 & 299.566 & 13815910.495 & -138151410.059 \\
\hline 13.480 & 1.820 & 9.897 & 18.610 & 66 & 11 & 299.144 & 135134840.309 & 135135339.449 \\
\hline 13.530 & 2.667 & 7.223 & 19.360 & 72 & 31 & 299.134 & 1381659.620 & 13817359.754 \\
\hline 13.580 & 3.077 & 6.973 & 24.310 & 71 & 12 & 299.574 & 138151410.641 & 138151910.211 \\
\hline 13.860 & 2.118 & 8.705 & 19.340 & 68 & 11 & 299.142 & 13517728.499 & 135171227.639 \\
\hline 14.120 & 2.167 & 8.988 & 19.800 & 68 & 21 & 359.543 & 135171916.509 & 135172516.050 \\
\hline 14.180 & 3.663 & 6.942 & 24.510 & 72 & 21 & 298.848 & 138164622.829 & 138165121.675 \\
\hline 14.340 & 3.537 & 8.039 & 24.090 & 72 & 12 & 299.134 & 138163830.898 & 138164330.029 \\
\hline 14.460 & 2.441 & 8.383 & 19.670 & 68 & 12 & 295.419 & 135171233.107 & 135171728.524 \\
\hline 14.680 & 2.075 & 9.396 & 20.020 & 68 & 22 & 358.295 & 135172522.344 & 135173120.639 \\
\hline 15.590 & 2.803 & 9.178 & 23.460 & 71 & 31 & 298.721 & 138153110.531 & 13815369.250 \\
\hline 15.920 & 2.348 & 10.320 & 23.120 & 71 & 41 & 299.130 & 138154240.824 & 138154739.951 \\
\hline 16.870 & 3.070 & 10.260 & 24.050 & 71 & 32 & 299.142 & 138153610.629 & 13815419.770 \\
\hline 17.280 & 3.476 & 8.016 & 25.170 & 72 & 22 & 299.146 & 138165122.508 & 138165621.652 \\
\hline 17.350 & 3.528 & 8.318 & 24.920 & 71 & 21 & 299.176 & 138152015.474 & 138152514.650 \\
\hline 18.740 & 2.429 & 11.660 & 25.960 & 71 & 42 & 287.025 & 138154746.530 & 138155233.554 \\
\hline
\end{tabular}




\begin{tabular}{|c|c|c|c|c|c|c|c|c|}
\hline Mean & Std & Min & $\operatorname{Max}$ & Tape & Event & Time Length & Start Time & End Time \\
\hline-18.707 & 16.069 & -49.147 & 11.988 & 69 & 32 & 293.222 & 135203546.647 & 135204039.869 \\
\hline-12.173 & 13.060 & -44.320 & 13.943 & 69 & 31 & 299.679 & 135203040.268 & 135203539.945 \\
\hline-9.380 & 21.110 & -42.529 & 84.554 & 72 & 32 & 261.823 & 138170406.242 & 138170828.064 \\
\hline-8.764 & 17.191 & -59.887 & 29.948 & 73 & 32 & 273.569 & 153120226.020 & 153120659.586 \\
\hline-8.217 & 20.059 & -61.922 & 36.723 & 67 & 22 & 298.959 & 135160430.791 & 135160929.750 \\
\hline-6.011 & 9.681 & -33.208 & 29.643 & 73 & 12 & 300.008 & 153114050.094 & 153114550.101 \\
\hline-5.836 & 13.320 & -44.902 & 39.618 & 72 & 11 & 299.750 & 138163330.441 & 138163830.191 \\
\hline-5.391 & 14.606 & -38.581 & 32.006 & 72 & 41 & 298.986 & 138170930.796 & 138171429.779 \\
\hline-4.946 & 12.819 & -60.038 & 39.482 & 68 & 12 & 295.419 & 135171233.107 & 135171728.524 \\
\hline-4.823 & 14.525 & -44.750 & 34.976 & 65 & 12 & 299.224 & 115093615.822 & 115094115.046 \\
\hline-4.701 & 10.666 & -36.548 & 19.310 & 66 & 22 & 299.046 & 135140740.831 & 135141239.875 \\
\hline-4.651 & 7.759 & -30.148 & 24.278 & 66 & 11 & 299.144 & 135134840.309 & 135135339.449 \\
\hline-4.643 & 12.266 & -41.561 & 18.184 & 70 & 31 & 299.305 & 135214520.167 & 135215019.470 \\
\hline-4.605 & 12.035 & -41.894 & 49.570 & 71 & 31 & 298.721 & 138153110.531 & 138153609.250 \\
\hline-4.505 & 14.273 & -54.215 & 25.399 & 73 & 31 & 298.996 & 153115720.314 & $153 \quad 120219.306$ \\
\hline-4.476 & 15.345 & -45.495 & 39.416 & 72 & 42 & 289.056 & 138171430.447 & 138171919.501 \\
\hline-4.310 & 12.474 & -39.142 & 32.795 & 69 & 22 & 224.076 & 135202445.731 & 135202829.803 \\
\hline-4.294 & 6.179 & -21.155 & 16.362 & 68 & 11 & 299.142 & 135170728.499 & 135171227.639 \\
\hline-4.009 & 9.937 & -32.995 & 19.905 & 72 & 12 & 299.134 & 138163830.898 & 138164330.029 \\
\hline-3.936 & 7.511 & -23.169 & 26.774 & 73 & 11 & 298.742 & 153113550.765 & 153114049.504 \\
\hline-3.908 & 11.488 & -42.591 & 16.975 & 75 & 22 & 289.576 & 206103305.072 & 206103754.646 \\
\hline-3.762 & 14.484 & -39.543 & 39.034 & 72 & 22 & 299.146 & 138165122.508 & 138165621.652 \\
\hline-3.733 & 11.372 & -39.556 & 31.808 & 71 & 21 & 299.176 & 138152015.474 & 138152514.650 \\
\hline-3.564 & 10.271 & -45.881 & 29.253 & 71 & 11 & 299.566 & 138150910.495 & 138151410.059 \\
\hline-3.408 & 9.636 & -24.546 & 26.585 & 68 & 22 & 358.295 & 135172522.344 & 135173120.639 \\
\hline-3.343 & 10.161 & -29.387 & 33.554 & 71 & 42 & 287.025 & 138154746.530 & 138155233.554 \\
\hline-3.226 & 7.591 & -25.176 & 20.263 & 67 & 11 & 299.499 & 135154740.226 & 135155239.723 \\
\hline-2.582 & 11.633 & -34.405 & 24.499 & 70 & 21 & 298.798 & 135213440.919 & 135213939.715 \\
\hline-2.534 & 13.779 & -37.591 & 43.729 & 66 & 21 & 299.234 & 135140240.759 & 135140739.991 \\
\hline-2.523 & 10.808 & -37.826 & 28.707 & 71 & 32 & 299.142 & 138153610.629 & 138154109.770 \\
\hline-2.521 & 8.241 & -28.071 & 28.271 & 66 & 31 & 299.449 & $135 \quad 14 \quad 14 \quad 10.599$ & 135141910.046 \\
\hline-2.505 & 8.253 & -23.971 & 31.304 & 73 & 21 & 299.151 & 153114640.756 & 153115139.904 \\
\hline-2.160 & 9.656 & -27.078 & 27.449 & 66 & 12 & 297.491 & 135135345.999 & 135135843.489 \\
\hline-2.040 & 12.161 & -29.877 & 50.383 & 68 & 21 & 359.543 & 135171916.509 & $1351725 \quad 16.050$ \\
\hline-2.004 & 9.790 & -29.317 & 22.145 & 66 & 32 & 298.883 & 135141910.642 & 135142409.523 \\
\hline-1.928 & 9.425 & -29.117 & 26.427 & 75 & 12 & 299.929 & 206101410.124 & 206101910.051 \\
\hline-1.866 & 10.681 & -29.271 & 29.085 & 71 & 12 & 299.574 & 138151410.641 & 138151910.211 \\
\hline-1.842 & 11.090 & -27.800 & 31.569 & 71 & 41 & 299.130 & 138154240.824 & 138154739.951 \\
\hline-1.547 & 10.153 & -30.393 & 24.790 & 67 & 32 & 299.149 & 135161600.526 & 135162059.672 \\
\hline-1.342 & 11.246 & -37.513 & 28.784 & 67 & 12 & 298.846 & 135155240.559 & 135155739.403 \\
\hline-1.288 & 9.404 & -34.605 & 17.311 & 73 & 22 & 292.729 & 153115146.543 & 153115639.272 \\
\hline-0.999 & 9.157 & -33.522 & 18.586 & 75 & 21 & 299.812 & 206102800.205 & 206103300.013 \\
\hline-0.831 & 7.695 & -19.384 & 21.201 & 65 & 22 & 299.370 & 115094740.501 & 115095239.869 \\
\hline-0.309 & 12.009 & -34.133 & 38.153 & 70 & 12 & 299.255 & 135212835.569 & 135213334.824 \\
\hline 0.154 & 8.769 & -24.567 & 32.897 & 75 & 11 & 299.439 & 206100910.688 & $2061014 \quad 10.124$ \\
\hline 0.334 & 10.821 & -39.895 & 24.731 & 73 & 42 & 299.532 & 153121250.225 & 153121749.755 \\
\hline 0.346 & 15.173 & -36.284 & 52.843 & 67 & 31 & 299.288 & 135161100.492 & 135161559.778 \\
\hline 0.955 & 11.421 & -26.618 & 47.750 & 70 & 22 & 254.371 & 135213945.583 & 135214359.952 \\
\hline 0.991 & 17.477 & -41.634 & 38.036 & 72 & 31 & 299.134 & 138165900.620 & 138170359.754 \\
\hline 1.301 & 12.758 & -48.193 & 43.275 & 70 & 42 & 293.820 & 135220205.718 & 135220659.537 \\
\hline 1.310 & 10.500 & -26.725 & 26.606 & 67 & 21 & 299.545 & 135155930.414 & 135160429.957 \\
\hline 1.424 & 11.874 & -40.536 & 47.525 & 65 & 11 & 299.439 & 115093115.541 & 115093614.980 \\
\hline 2.277 & 10.789 & -23.440 & 39.917 & 70 & 41 & 299.230 & 135215700.617 & 135220159.845 \\
\hline 3.090 & 14.333 & -33.696 & 33.616 & 70 & 32 & 299.151 & 135215020.307 & 135215519.458 \\
\hline 3.791 & 15.042 & -27.614 & 37.010 & 73 & 41 & 298.510 & 153120750.870 & 153121249.378 \\
\hline 4.119 & 9.754 & -21.976 & 56.875 & 65 & 21 & 299.355 & 115094240.316 & 115094739.669 \\
\hline 4.309 & 8.214 & -21.973 & 29.424 & 70 & 11 & 299.601 & 135212335.137 & 135212834.737 \\
\hline 5.564 & 13.342 & -33.205 & 57.035 & 72 & 21 & 298.848 & 138164622.829 & 138165121.675 \\
\hline 13.549 & 20.688 & -30.570 & 66.412 & 69 & 21 & 254.521 & 135201945.149 & 135202359.668 \\
\hline
\end{tabular}


458: Total Pressure Probe at $86 \%$ Span (psi)

\begin{tabular}{|c|c|c|c|c|c|c|c|c|}
\hline Mean & Std & Min & $\operatorname{Max}$ & Tape & Event & Time Length & Start Time & End Time \\
\hline 0.073 & 0.008 & -0.890 & 1.212 & 73 & 12 & 300.008 & 153114050.094 & 153114550.101 \\
\hline 0.074 & 0.005 & -0.402 & 0.100 & 73 & 22 & 292.729 & 153115146.543 & 153115639.272 \\
\hline 0.074 & 0.005 & -0.405 & 0.102 & 73 & 11 & 298.742 & 153113550.765 & 153114049.504 \\
\hline 0.074 & 0.005 & -0.403 & 0.831 & 73 & 21 & 299.151 & 153114640.756 & 153115139.904 \\
\hline 0.075 & 0.008 & -0.403 & 0.834 & 73 & 32 & 273.569 & 15312226.020 & 15312659.586 \\
\hline 0.075 & 0.005 & -0.404 & 0.099 & 73 & 31 & 298.996 & 153115720.314 & 15312219.306 \\
\hline 0.078 & 0.005 & -0.396 & 0.111 & 73 & 42 & 299.532 & 153121250.225 & 153121749.755 \\
\hline 0.079 & 0.011 & -0.465 & 1.256 & 75 & 22 & 289.576 & 20610335.072 & 206103754.646 \\
\hline 0.079 & 0.007 & -0.386 & 0.113 & 70 & 11 & 299.601 & 135212335.137 & 135212834.737 \\
\hline 0.079 & 0.005 & -0.399 & 0.103 & 73 & 41 & 298.510 & 15312750.870 & 153121249.378 \\
\hline 0.080 & 0.010 & -0.489 & 0.967 & 65 & 22 & 299.370 & 11594740.501 & 11595239.869 \\
\hline 0.080 & 0.007 & -0.372 & 0.759 & 70 & 12 & 299.255 & 135212835.569 & 135213334.824 \\
\hline 0.080 & 0.024 & -0.626 & 1.180 & 69 & 22 & 224.076 & 135202445.731 & 135202829.803 \\
\hline 0.080 & 0.006 & -0.381 & 0.111 & 70 & 41 & 299.230 & $\begin{array}{lll}1352157 & .617\end{array}$ & 13522159.845 \\
\hline 0.080 & 0.009 & -0.977 & 0.718 & 75 & 12 & 299.929 & 206101410.124 & 206101910.051 \\
\hline 0.081 & 0.025 & -0.951 & 0.981 & 69 & 21 & 254.521 & 135201945.149 & 135202359.668 \\
\hline 0.081 & 0.006 & 0.058 & 0.113 & 67 & 22 & 298.959 & 13516430.791 & 13516929.750 \\
\hline 0.081 & 0.015 & -0.464 & 0.765 & 70 & 22 & 254.371 & 135213945.583 & 135214359.952 \\
\hline 0.081 & 0.007 & -0.372 & 0.113 & 70 & 32 & 299.151 & 135215020.307 & 135215519.458 \\
\hline 0.081 & 0.010 & -0.995 & 1.263 & 75 & 21 & 299.812 & 2061028.205 & $2061033 \quad .013$ \\
\hline 0.081 & 0.007 & -0.387 & 0.770 & 70 & 21 & 298.798 & 135213440.919 & 135213939.715 \\
\hline 0.081 & 0.006 & -0.403 & 0.871 & 70 & 31 & 299.305 & 135214520.167 & 135215019.470 \\
\hline 0.081 & 0.017 & -0.507 & 0.933 & 72 & 42 & 289.056 & 138171430.447 & 138171919.501 \\
\hline 0.081 & 0.007 & -0.227 & 0.114 & 70 & 42 & 293.820 & 1352225.718 & 13522659.537 \\
\hline 0.082 & 0.023 & -0.494 & 0.979 & 65 & 12 & 299.224 & 11593615.822 & 11594115.046 \\
\hline 0.082 & 0.008 & -0.438 & 0.766 & 65 & 21 & 299.355 & 11594240.316 & 11594739.669 \\
\hline 0.082 & 0.020 & -0.510 & 0.925 & 72 & 32 & 261.823 & 1381746.242 & 13817828.064 \\
\hline 0.082 & 0.011 & -0.434 & 0.993 & 67 & 31 & 299.288 & $1351611 \quad .492$ & 135161559.778 \\
\hline 0.082 & 0.016 & -0.486 & 1.289 & 66 & 32 & 298.883 & 135141910.642 & 13514249.523 \\
\hline 0.082 & 0.009 & -0.190 & 0.781 & 67 & 32 & 299.149 & 1351616.526 & 135162059.672 \\
\hline 0.082 & 0.022 & -0.483 & 0.986 & 69 & 32 & 293.222 & 135203546.647 & 135204039.869 \\
\hline 0.082 & 0.008 & -0.461 & 0.130 & 67 & 12 & 298.846 & 135155240.559 & 135155739.403 \\
\hline 0.083 & 0.014 & -1.007 & 1.265 & 75 & 11 & 299.439 & 20610910.688 & 206101410.124 \\
\hline 0.083 & 0.007 & -0.225 & 0.120 & 65 & 11 & 299.439 & 11593115.541 & 11593614.980 \\
\hline 0.083 & 0.007 & -0.224 & 0.995 & 67 & 21 & 299.545 & 135155930.414 & 13516429.957 \\
\hline 0.083 & 0.017 & -0.484 & 1.344 & 69 & 31 & 299.679 & 135203040.268 & 135203539.945 \\
\hline 0.084 & 0.015 & -0.938 & 0.983 & 66 & 31 & 299.449 & 135141410.599 & 135141910.046 \\
\hline 0.084 & 0.007 & -0.225 & 0.782 & 67 & 11 & 299.499 & 135154740.226 & 135155239.723 \\
\hline 0.084 & 0.013 & -0.432 & 1.258 & 66 & 22 & 299.046 & $135 \cdot 14740.831$ & 135141239.875 \\
\hline 0.085 & 0.014 & -0.452 & 1.308 & 72 & 11 & 299.750 & 138163330.441 & 138163830.191 \\
\hline 0.086 & 0.021 & -0.502 & 0.995 & 71 & 11 & 299.566 & 13815910.495 & 138151410.059 \\
\hline 0.086 & 0.014 & -0.907 & 0.988 & 66 & 21 & 299.234 & 13514240.759 & 13514739.991 \\
\hline 0.086 & 0.020 & -0.505 & 1.038 & 72 & 31 & 299.134 & 1381659.620 & 13817359.754 \\
\hline 0.086 & 0.018 & -0.489 & 0.980 & 72 & 21 & 298.848 & 138164622.829 & 138165121.675 \\
\hline 0.086 & 0.018 & -0.500 & 0.901 & 71 & 12 & 299.574 & 138151410.641 & 138151910.211 \\
\hline 0.086 & 0.022 & -0.652 & 0.996 & 72 & 41 & 298.986 & 13817930.796 & 138171429.779 \\
\hline 0.088 & 0.018 & -0.510 & 0.751 & 72 & 12 & 299.134 & 138163830.898 & 138164330.029 \\
\hline 0.088 & 0.022 & -0.966 & 0.995 & 68 & 22 & 358.295 & 135172522.344 & 135173120.639 \\
\hline 0.088 & 0.015 & -0.913 & 1.010 & 66 & 12 & 297.491 & 135135345.999 & 135135843.489 \\
\hline 0.089 & 0.010 & -0.937 & 0.140 & 68 & 11 & 299.142 & 13517728.499 & 135171227.639 \\
\hline 0.089 & 0.013 & -0.429 & 0.172 & 68 & 21 & 359.543 & 135171916.509 & 135172516.050 \\
\hline 0.090 & 0.015 & -1.013 & 0.776 & 71 & 41 & 299.130 & 138154240.824 & 138154739.951 \\
\hline 0.090 & 0.013 & -0.282 & 0.155 & 68 & 12 & 295.419 & 135171233.107 & 135171728.524 \\
\hline 0.092 & 0.020 & -0.511 & 0.780 & 72 & 22 & 299.146 & 138165122.508 & 138165621.652 \\
\hline 0.093 & 0.018 & -0.489 & 0.890 & 71 & 32 & 299.142 & 138153610.629 & 13815419.770 \\
\hline 0.093 & 0.019 & -0.959 & 0.905 & 71 & 31 & 298.721 & 138153110.531 & 13815369.250 \\
\hline 0.093 & 0.018 & -0.496 & 0.896 & 71 & 42 & 287.025 & 138154746.530 & 138155233.554 \\
\hline 0.094 & 0.020 & -0.930 & 0.900 & 71 & 21 & 299.176 & 138152015.474 & 138152514.650 \\
\hline 0.096 & 0.013 & -0.554 & 1.003 & 66 & 11 & 299.144 & 135134840.309 & 135135339.449 \\
\hline
\end{tabular}


934: Total Pressure Probe at 67.3\% Span (psi)

\begin{tabular}{|c|c|c|c|c|c|c|c|c|}
\hline Mean & Std & Min & $\operatorname{Max}$ & Tape & Event & Time Length & Start Time & End Time \\
\hline 0.049 & 0.006 & -0.516 & 0.625 & 75 & 12 & 299.929 & $2061014 \quad 10.124$ & 206101910.051 \\
\hline 0.049 & 0.005 & -0.536 & 0.074 & 70 & 41 & 299.230 & $1352157 \quad .617$ & 13522159.845 \\
\hline 0.049 & 0.005 & -1.077 & 0.074 & 65 & 22 & 299.370 & 11594740.501 & 11595239.869 \\
\hline 0.050 & 0.004 & 0.032 & 0.072 & 67 & 22 & 298.959 & 13516430.791 & 13516929.750 \\
\hline 0.050 & 0.005 & 0.029 & 0.076 & $\overline{73}$ & 32 & 273.569 & 15312226.020 & 15312659.586 \\
\hline 0.050 & 0.006 & -0.529 & 0.626 & 75 & 22 & 289.576 & 20610335.072 & 206103754.646 \\
\hline 0.050 & 0.007 & 0.022 & 0.643 & 73 & 42 & 299.532 & $153 \quad 121250.225$ & 153121749.755 \\
\hline 0.050 & 0.006 & -0.774 & 0.258 & 70 & 42 & 293.820 & 1352225.718 & 13522659.537 \\
\hline 0.050 & 0.005 & 0.030 & 0.241 & 70 & 32 & 299.151 & 135215020.307 & 135215519.458 \\
\hline 0.050 & 0.005 & -0.887 & 0.541 & 70 & 31 & 299.305 & 135214520.167 & 135215019.470 \\
\hline 0.050 & 0.006 & -0.581 & 0.088 & 65 & 21 & 299.355 & 11594240.316 & 11594739.669 \\
\hline 0.050 & 0.005 & 0.022 & 0.076 & 70 & 11 & 299.601 & 135212335.137 & 135212834.737 \\
\hline 0.051 & 0.005 & 0.022 & 0.082 & 70 & 22 & 254.371 & 135213945.583 & 135214359.952 \\
\hline 0.051 & 0.007 & 0.024 & 0.083 & 73 & 41 & 298.510 & 15312750.870 & 153121249.378 \\
\hline 0.051 & 0.007 & -0.569 & 1.127 & 72 & 42 & 289.056 & $13817 \quad 1430.447$ & 138171919.501 \\
\hline 0.051 & 0.005 & 0.018 & 0.259 & 70 & 21 & 298.798 & 135213440.919 & 135213939.715 \\
\hline 0.051 & 0.008 & -1.097 & 1.189 & 75 & 11 & 299.439 & 20610910.688 & 206101410.124 \\
\hline 0.051 & 0.006 & -0.529 & 0.705 & 75 & 21 & 299.812 & $2061028 \quad .205$ & $2061033 \quad .013$ \\
\hline 0.051 & 0.007 & 0.027 & 0.087 & 73 & 31 & 298.996 & 153115720.314 & 15312219.306 \\
\hline 0.051 & 0.006 & 0.025 & 0.086 & 67 & 12 & 298.846 & 135155240.559 & 135155739.403 \\
\hline 0.051 & 0.006 & -1.025 & 0.537 & 70 & 12 & 299.255 & 135212835.569 & 135213334.824 \\
\hline 0.051 & 0.006 & -0.589 & 0.562 & 67 & 21 & 299.545 & 135155930.414 & 13516429.957 \\
\hline 0.051 & 0.006 & -1.082 & 0.113 & 65 & 12 & 299.224 & 11593615.822 & 11594115.046 \\
\hline 0.051 & 0.006 & 0.023 & 0.567 & 69 & 22 & 224.076 & 135202445.731 & 135202829.803 \\
\hline 0.052 & 0.010 & 0.018 & 0.099 & 69 & 21 & 254.521 & 135201945.149 & 135202359.668 \\
\hline 0.052 & 0.005 & 0.027 & 0.081 & 66 & 32 & 298.883 & 135141910.642 & 13514249.523 \\
\hline 0.052 & 0.006 & -0.611 & 0.084 & 65 & II & 299.439 & 11593115.541 & 11593614.980 \\
\hline 0.052 & 0.006 & 0.028 & 0.080 & 66 & 22 & 299.046 & 13514740.831 & 135141239.875 \\
\hline 0.052 & 0.006 & -0.882 & 0.554 & 67 & 11 & 299.499 & 135154740.226 & 135155239.723 \\
\hline 0.053 & 0.008 & -0.129 & 0.083 & 69 & 32 & 293.222 & 135203546.647 & 135204039.869 \\
\hline 0.053 & 0.007 & -0.845 & 0.091 & 66 & 21 & 299.234 & 13514240.759 & 13514739.991 \\
\hline 0.053 & 0.007 & -1.080 & 0.083 & 66 & 31 & 299.449 & 135141410.599 & 135141910.046 \\
\hline 0.053 & 0.008 & -0.002 & 0.091 & 67 & 31 & 299.288 & $1351611 \quad .492$ & 135161559.778 \\
\hline 0.054 & 0.007 & 0.026 & 1.187 & 73 & 22 & 292.729 & 153115146.543 & 153115639.272 \\
\hline 0.054 & 0.007 & -0.593 & 0.088 & 67 & 32 & 299.149 & $1351616 \quad .526$ & 135162059.672 \\
\hline 0.054 & 0.006 & -0.843 & 0.573 & 69 & 31 & 299.679 & 135203040.268 & 135203539.945 \\
\hline 0.054 & 0.012 & 0.009 & 0.113 & 72 & 32 & 261.823 & 1381746.242 & 13817828.064 \\
\hline 0.055 & 0.013 & -0.612 & 0.116 & 71 & 21 & 299.176 & 138152015.474 & 138152514.650 \\
\hline 0.055 & 0.013 & -0.553 & 0.578 & 71 & 41 & 299.130 & 138154240.824 & 138154739.951 \\
\hline 0.055 & 0.012 & -0.003 & 0.157 & 72 & 41 & 298.986 & 13817930.796 & 138171429.779 \\
\hline 0.056 & 0.007 & -0.597 & 0.091 & 66 & 12 & 297.491 & 135135345.999 & 135135843.489 \\
\hline 0.056 & 0.010 & 0.010 & 0.555 & 72 & 11 & 299.750 & 138163330.441 & 138163830.191 \\
\hline 0.057 & 0.008 & 0.020 & 0.100 & 73 & 12 & 300.008 & 153114050.094 & 153114550.101 \\
\hline 0.057 & 0.007 & 0.018 & 1.170 & 73 & 21 & 299.151 & 153114640.756 & 153115139.904 \\
\hline 0.057 & 0.013 & -0.879 & 0.126 & 72 & 21 & 298.848 & 138164622.829 & 138165121.675 \\
\hline 0.058 & 0.013 & 0.012 & 0.115 & 72 & 31 & 299.134 & $1381659 \quad .620$ & 13817359.754 \\
\hline 0.058 & 0.010 & -1.051 & 0.541 & 68 & 11 & 299.142 & 13517728.499 & 135171227.639 \\
\hline 0.059 & 0.009 & -0.623 & 0.104 & 68 & 22 & 358.295 & 135172522.344 & 135173120.639 \\
\hline 0.059 & 0.010 & 0.004 & 0.104 & 68 & 12 & 295.419 & $135 \quad 17 \quad 1233.107$ & 135171728.524 \\
\hline 0.059 & 0.009 & -0.833 & 0.112 & 71 & 11 & 299.566 & 13815910.495 & 138151410.059 \\
\hline 0.059 & 0.010 & 0.006 & 0.548 & 68 & 21 & 359.543 & 135171916.509 & 135172516.050 \\
\hline 0.059 & 0.012 & -0.554 & 0.590 & 71 & 32 & 299.142 & 138153610.629 & 13815419.770 \\
\hline 0.059 & 0.012 & -0.580 & 0.574 & 71 & 31 & 298.721 & 138153110.531 & 13815369.250 \\
\hline 0.059 & 0.010 & 0.017 & 0.584 & 71 & 12 & 299.574 & $1381514 \quad 10.641$ & 138151910.211 \\
\hline 0.060 & 0.011 & 0.019 & 0.204 & 72 & 12 & 299.134 & 138163830.898 & 138164330.029 \\
\hline 0.060 & 0.008 & 0.025 & 0.128 & 73 & 11 & 298.742 & 153113550.765 & 153114049.504 \\
\hline 0.061 & 0.007 & -0.052 & 0.093 & 66 & 11 & 299.144 & 135134840.309 & 135135339.449 \\
\hline 0.063 & 0.016 & 0.003 & 0.947 & 72 & 22 & 299.146 & 138165122.508 & 138165621.652 \\
\hline 0.064 & 0.013 & -0.559 & 0.618 & 71 & 42 & 287.025 & 138154746.530 & 138155233.554 \\
\hline
\end{tabular}


862: Total Pressure Probe at 50.6\% Span (psi)

\begin{tabular}{|c|c|c|c|c|c|c|c|c|}
\hline Mean & Std & Min & Max & Tape & Event & Time Length & Start Time & End Time \\
\hline 0.028 & 0.005 & 0.009 & 0.051 & 73 & 42 & 299.532 & 153121250.225 & 153121749.755 \\
\hline 0.028 & 0.003 & -0.185 & 0.242 & 67 & 22 & 298.959 & 13516430.791 & 13516929.750 \\
\hline 0.028 & 0.003 & -0.181 & 0.242 & 65 & 22 & 299.370 & 11594740.501 & 11595239.869 \\
\hline 0.028 & 0.005 & 0.012 & 0.053 & 73 & 41 & 298.510 & 15312750.870 & 153121249.378 \\
\hline 0.029 & 0.005 & -0.392 & 0.247 & 75 & 22 & 289.576 & 20610335.072 & 206103754.646 \\
\hline 0.029 & 0.003 & -0.183 & 0.046 & 70 & 31 & 299.305 & 135214520.167 & 135215019.470 \\
\hline 0.029 & 0.004 & -0.188 & 0.054 & 70 & 32 & 299.151 & 135215020.307 & 135215519.458 \\
\hline 0.029 & 0.004 & -0.185 & 0.244 & 73 & 32 & 273.569 & 15312226.020 & 15312659.586 \\
\hline 0.029 & 0.005 & -0.393 & 0.243 & 65 & 21 & 299.355 & 11594240.316 & 11594739.669 \\
\hline 0.030 & 0.004 & -0.184 & 0.053 & 70 & 41 & 299.230 & $1352157 \quad .617$ & $13522 \quad 159.845$ \\
\hline 0.030 & 0.005 & -0.185 & 0.345 & 75 & 12 & 299.929 & 206101410.124 & 206101910.051 \\
\hline 0.030 & 0.004 & -0.003 & 0.238 & 67 & 21 & 299.545 & 135155930.414 & 13516429.957 \\
\hline 0.030 & 0.005 & -0.185 & 0.246 & 75 & 21 & 299.812 & 2061028.205 & $2061033 \quad .013$ \\
\hline 0.030 & 0.004 & -0.185 & 0.054 & 70 & 21 & 298.798 & 135213440.919 & 135213939.715 \\
\hline 0.030 & 0.004 & -0.393 & 0.242 & 65 & 11 & 299.439 & 11593115.541 & 11593614.980 \\
\hline 0.030 & 0.004 & -0.003 & 0.055 & 70 & 11 & 299.601 & 135212335.137 & 135212834.737 \\
\hline 0.030 & 0.005 & -0.197 & 0.248 & 73 & 31 & 298.996 & 153115720.314 & 15312219.306 \\
\hline 0.030 & 0.005 & -0.187 & 0.242 & 70 & 22 & 254.371 & 135213945.583 & 135214359.952 \\
\hline 0.031 & 0.004 & 0.009 & 0.054 & 70 & 42 & 293.820 & 1352225.718 & 13522659.537 \\
\hline 0.031 & 0.004 & 0.007 & 0.059 & 73 & 22 & 292.729 & 153115146.543 & 153115639.272 \\
\hline 0.031 & 0.008 & -0.189 & 0.245 & 69 & 21 & 254.521 & 135201945.149 & 135202359.668 \\
\hline 0.031 & 0.005 & -0.186 & 0.242 & 65 & 12 & 299.224 & 11593615.822 & 11594115.046 \\
\hline 0.031 & 0.006 & -0.185 & 0.247 & 72 & 42 & 289.056 & 138171430.447 & 138171919.501 \\
\hline 0.031 & 0.006 & -0.180 & 0.242 & 69 & 32 & 293.222 & 135203546.647 & 135204039.869 \\
\hline 0.031 & 0.005 & -0.288 & 0.248 & 75 & 11 & 299.439 & 20610910.688 & 206101410.124 \\
\hline 0.031 & 0.005 & -0.183 & 0.242 & 69 & 22 & 224.076 & 135202445.731 & 135202829.803 \\
\hline 0.031 & 0.005 & -0.180 & 0.243 & 69 & 31 & 299.679 & 135203040.268 & 135203539.945 \\
\hline 0.031 & 0.004 & 0.011 & 0.243 & 70 & 12 & 299.255 & 135212835.569 & 135213334.824 \\
\hline 0.031 & 0.006 & -0.383 & 0.242 & 66 & 21 & 299.234 & 13514240.759 & 13514739.991 \\
\hline 0.032 & 0.004 & 0.010 & 0.058 & 66 & 32 & 298.883 & 135141910.642 & 13514249.523 \\
\hline 0.032 & 0.005 & -0.178 & 0.242 & 66 & 22 & 299.046 & 13514740.831 & 135141239.875 \\
\hline 0.032 & 0.005 & 0.006 & 0.242 & 67 & 12 & 298.846 & 135155240.559 & 135155739.403 \\
\hline 0.032 & 0.004 & -0.183 & 0.058 & 67 & 11 & 299.499 & 135154740.226 & 135155239.723 \\
\hline 0.033 & 0.006 & -0.240 & 0.242 & 66 & 31 & 299.449 & 135141410.599 & 135141910.046 \\
\hline 0.033 & 0.007 & -0.013 & 0.244 & 67 & 31 & 299.288 & 1351611.492 & 135161559.778 \\
\hline 0.033 & 0.005 & -0.188 & 0.251 & 73 & 21 & 299.151 & 153114640.756 & 153115139.904 \\
\hline 0.034 & 0.009 & 0.001 & 0.079 & 72 & 32 & 261.823 & 1381746.242 & 13817828.064 \\
\hline 0.034 & 0.006 & -0.179 & 0.242 & 66 & 12 & 297.491 & 135135345.999 & 135135843.489 \\
\hline 0.034 & 0.006 & -0.190 & 0.242 & 67 & 32 & 299.149 & $1351616 \quad .526$ & 135162059.672 \\
\hline 0.035 & 0.006 & -0.184 & 0.246 & 73 & 12 & 300.008 & 153114050.094 & 153114550.101 \\
\hline 0.035 & 0.009 & -0.185 & 0.249 & 72 & 11 & 299.750 & 138163330.441 & 138163830.191 \\
\hline 0.035 & 0.011 & -0.193 & 0.244 & 72 & 41 & 298.986 & 13817930.796 & 138171429.779 \\
\hline 0.036 & 0.008 & -0.188 & 0.246 & 71 & 11 & 299.566 & $13815 \quad 910.495$ & 138151410.059 \\
\hline .0 .037 & 0.009 & -0.193 & 0.261 & 71 & 12 & 299.574 & 138151410.641 & 138151910.211 \\
\hline 0.037 & 0.006 & -0.175 & 0.246 & 66 & 11 & 299.144 & 135134840.309 & 135135339.449 \\
\hline 0.037 & 0.006 & -0.389 & 0.250 & 73 & 11 & 298.742 & 153113550.765 & 153114049.504 \\
\hline 0.037 & 0.011 & -0.375 & 0.253 & 72 & 31 & 299.134 & $1381659 \quad .620$ & $13817 \quad 359.754$ \\
\hline 0.038 & 0.007 & -0.280 & 0.246 & 68 & 11 & 299.142 & 13517728.499 & 135171227.639 \\
\hline 0.038 & 0.010 & -0.184 & 0.245 & 72 & 21 & 298.848 & 138164622.829 & 138165121.675 \\
\hline 0.038 & 0.009 & -0.007 & 0.095 & 72 & 12 & 299.134 & 138163830.898 & 138164330.029 \\
\hline 0.039 & 0.009 & -0.209 & 0.253 & 68 & 21 & 359.543 & 135171916.509 & 135172516.050 \\
\hline 0.039 & 0.009 & -0.389 & 0.250 & 68 & 12 & 295.419 & 135171233.107 & 135171728.524 \\
\hline 0.039 & 0.008 & -0.207 & 0.447 & 68 & 22 & 358.295 & 135172522.344 & 135173120.639 \\
\hline 0.040 & 0.010 & -0.378 & 0.251 & 71 & 41 & 299.130 & 138154240.824 & 138154739.951 \\
\hline 0.042 & 0.010 & -0.391 & 0.252 & 71 & 31 & 298.721 & 138153110.531 & 13815369.250 \\
\hline 0.043 & 0.010 & -0.178 & 0.254 & 71 & 32 & 299.142 & 138153610.629 & 13815419.770 \\
\hline 0.044 & 0.013 & -0.186 & 0.247 & 72 & 22 & 299.146 & 138165122.508 & 138165621.652 \\
\hline 0.045 & 0.013 & -0.364 & 0.444 & 71 & 21 & 299.176 & 138152015.474 & 138152514.650 \\
\hline 0.045 & 0.011 & -0.189 & 0.253 & 71 & 42 & 287.025 & 138154746.530 & 138155233.554 \\
\hline
\end{tabular}


832: Total Pressure Probe at 34\% Span (psi)

\begin{tabular}{|c|c|c|c|c|c|c|c|c|}
\hline Mean & Std & Min & $\operatorname{Max}$ & Tape & Event & Time Length & Start Time & End Time \\
\hline 0.013 & 0.002 & -0.181 & 0.025 & 67 & 22 & 298.959 & 13516430.791 & 13516929.750 \\
\hline 0.013 & 0.003 & -0.416 & 0.236 & 65 & 22 & 299.370 & 11594740.501 & 11595239.869 \\
\hline 0.013 & 0.003 & -0.003 & 0.029 & 73 & 42 & 299.532 & 153121250.225 & 153121749.755 \\
\hline 0.014 & 0.004 & -0.417 & 0.242 & 65 & 21 & 299.355 & 11594240.316 & 11594739.669 \\
\hline 0.014 & 0.003 & -0.417 & 0.240 & 65 & 11 & 299.439 & 11593115.541 & 11593614.980 \\
\hline 0,014 & 0.003 & 0.002 & 0.241 & 73 & 41 & 298.510 & 15312750.870 & 153121249.378 \\
\hline 0.014 & 0.003 & -0.204 & 0.237 & 73 & 32 & 273.569 & 15312226.020 & 15312659.586 \\
\hline 0.014 & 0.003 & -0.206 & 0.028 & 70 & 31 & 299.305 & 135214520.167 & 135215019.470 \\
\hline 0.014 & 0.004 & -0.204 & 0.454 & 65 & 12 & 299.224 & 11593615.822 & 11594115.046 \\
\hline 0.015 & 0.003 & -0.019 & 0.031 & 67 & 21 & 299.545 & 135155930.414 & 13516429.957 \\
\hline 0.015 & 0.003 & -0.004 & 0.230 & 70 & 32 & 299.151 & 135215020.307 & 135215519.458 \\
\hline 0.015 & 0.004 & -0.200 & 0.403 & $\overline{75}$ & 22 & 289.576 & 20610335.072 & 206103754.646 \\
\hline 0.015 & 0.003 & -0.207 & 0.233 & 70 & 41 & 299.230 & $\begin{array}{ll}1352157 & .617\end{array}$ & 13522159.845 \\
\hline 0.015 & 0.006 & -0.207 & 0.356 & 69 & 21 & 254.521 & 135201945.149 & 135202359.668 \\
\hline 0.015 & 0.003 & -0.022 & 0.031 & 70 & 11 & 299.601 & 135212335.137 & 135212834.737 \\
\hline 0.015 & 0.004 & -0.210 & 0.031 & 70 & 21 & 298.798 & 135213440.919 & 135213939.715 \\
\hline 0.015 & 0.004 & -0.203 & 0.353 & 75 & 12 & 299.929 & 206101410.124 & 206101910.051 \\
\hline 0.015 & 0.004 & -0.201 & 0.239 & 73 & 31 & 298.996 & 153115720.314 & 15312219.306 \\
\hline 0.016 & 0.003 & 0.001 & 0.031 & 70 & 42 & 293.820 & 1352225.718 & 13522659.537 \\
\hline 0.016 & 0.004 & -0.209 & 0.236 & 70 & 22 & 254.371 & 135213945.583 & 135214359.952 \\
\hline 0.016 & 0.004 & -0.202 & 0.244 & 75 & 21 & 299.812 & $2061028 \quad .205$ & $2061033 \quad .013$ \\
\hline 0.016 & 0.003 & 0.000 & 0.035 & 73 & 22 & 292.729 & 153115146.543 & 153115639.272 \\
\hline 0.016 & 0.005 & -0.201 & 0.233 & 69 & 32 & 293.222 & 135203546.647 & 135204039.869 \\
\hline 0.016 & 0.005 & -0.209 & 0.234 & 66 & 21 & 299.234 & 13514240.759 & 13514739.991 \\
\hline 0.016 & 0.004 & -0.202 & 0.231 & 69 & 22 & 224.076 & 135202445.731 & 135202829.803 \\
\hline 0.016 & 0.005 & -0.384 & 0.240 & 72 & 42 & 289.056 & 138171430.447 & $\begin{array}{lllll}38 & 17 & 19 & 19.501\end{array}$ \\
\hline 0,016 & 0.004 & -0.200 & 0.234 & 69 & 31 & 299.679 & 135203040.268 & 135203539.945 \\
\hline 0.016 & 0.004 & -0.199 & 0.315 & 75 & 11 & 299.439 & 20610910.688 & 206101410.124 \\
\hline 0.017 & 0.003 & -0.006 & 0.236 & 70 & 12 & 299.255 & 135212835.569 & 135213334.824 \\
\hline 0.017 & 0.004 & -0.203 & 0.235 & 66 & 22 & 299.046 & 13514740.831 & 135141239.875 \\
\hline 0.017 & 0.003 & -0.001 & 0.039 & 67 & 12 & 298.846 & 135155240.559 & 135155739.403 \\
\hline 0.017 & 0.004 & -0.207 & 0.238 & 67 & 11 & 299.499 & 135154740.226 & 135155239.723 \\
\hline 0.017 & 0.003 & -0.001 & 0.231 & 66 & 32 & 298.883 & 135141910.642 & 13514249.523 \\
\hline 0.018 & 0.005 & -0.027 & 0.045 & 67 & 31 & 299.288 & $\begin{array}{ll}1351611 & .492\end{array}$ & 135161559.778 \\
\hline 0.018 & 0.005 & -0.207 & 0.447 & 66 & 31 & 299.449 & 135141410.599 & 135141910.046 \\
\hline 0.018 & 0.004 & -0.308 & 0.242 & 73 & 21 & 299.151 & 153114640.756 & 153115139.904 \\
\hline 0.018 & 0.008 & -0.013 & 0.055 & 72 & 32 & 261.823 & 1381746.242 & 13817828.064 \\
\hline 0.019 & 0.005 & -0.203 & 0.236 & 66 & 12 & 297.491 & 135135345.999 & 135135843.489 \\
\hline 0.019 & 0.004 & -0.009 & 0.234 & 67 & 32 & 299.149 & 1351616.526 & 135162059.672 \\
\hline 0.020 & 0.005 & -0.203 & 0.244 & 73 & 12 & 300.008 & 153114050.094 & 153114550.101 \\
\hline 0.020 & 0.008 & -0.203 & 0.239 & 72 & 11 & 299.750 & 138163330.441 & 138163830.191 \\
\hline 0.020 & 0.009 & -0.207 & 0.243 & 72 & 41 & 298.986 & 13817930.796 & 138171429.779 \\
\hline 0.021 & 0.005 & -0.206 & 0.237 & 66 & 11 & 299.144 & 135134840.309 & 135135339.449 \\
\hline 0.021 & 0.008 & -0.235 & 0.241 & 71 & 11 & 299.566 & 13815910.495 & 138151410.059 \\
\hline 0.021 & 0.005 & -0.204 & 0.242 & $7 \overline{3}$ & 11 & 298.742 & 153113550.765 & 153114049.504 \\
\hline 0.022 & 0.008 & -0.205 & 0.251 & 71 & 12 & 299.574 & $1381514 \quad 10.641$ & $1381519 \quad 10.211$ \\
\hline 0.022 & 0.009 & -0.204 & 0.242 & 72 & 31 & 299.134 & $1381659 \quad .620$ & 13817359.754 \\
\hline 0,022 & 0.006 & -0.417 & 0.443 & 68 & 11 & 299.142 & 13517728.499 & 135171227.639 \\
\hline 0.023 & 0.009 & -0.203 & 0.239 & 72 & 21 & 298.848 & 138164622.829 & 138165121.675 \\
\hline 0.023 & 0.008 & -0.096 & 0.069 & 72 & 12 & 299.134 & 138163830.898 & 138164330.029 \\
\hline 0.024 & 0.007 & -0.425 & 0.238 & 68 & 21 & 359.543 & 135171916.509 & 135172516.050 \\
\hline 0.024 & 0.007 & -0.252 & 0.055 & 68 & 12 & 295.419 & 135171233.107 & 135171728.524 \\
\hline 0.025 & 0.007 & -0.317 & 0.242 & 68 & 22 & 358.295 & 135172522.344 & 135173120.639 \\
\hline 0.025 & 0.009 & -0.202 & 0.290 & 71 & 41 & 299.130 & 138154240.824 & 138154739.951 \\
\hline 0.026 & 0.009 & -0.206 & 0.248 & 71 & 31 & 298.721 & 138153110.531 & 13815369.250 \\
\hline 0.028 & 0.011 & -0.211 & 0.241 & 72 & 22 & 299.146 & 138165122.508 & 138165621.652 \\
\hline 0.028 & 0.010 & -0.209 & 0.448 & 71 & 32 & 299.142 & 138153610.629 & 13815419.770 \\
\hline 0.028 & 0.011 & -0.387 & 0.453 & 71 & 21 & 299.176 & 138152015.474 & 138152514.650 \\
\hline 0.030 & 0.010 & -0.287 & 0.245 & 71 & 42 & 287.025 & 138154746.530 & 138155233.554 \\
\hline
\end{tabular}


461: Instrumented Blade Pitch Angle (deg)

\begin{tabular}{|c|c|c|c|c|c|c|c|c|}
\hline Mean & Std & Min & Max & Tape & Event & Time Length & Start Time & End Time \\
\hline 8.611 & 0.558 & 7.012 & 15.090 & 65 & 11 & 299.439 & 11593115.541 & 11593614.980 \\
\hline 8.812 & 0.493 & -75.220 & 40.290 & 65 & 22 & 299.370 & 11594740.501 & 11595239.869 \\
\hline 8.874 & 0.427 & -75.300 & 15.180 & 65 & 21 & 299.355 & 11594240.316 & 11594739.669 \\
\hline 8.880 & 1.479 & -75.260 & 40.370 & 65 & 12 & 299.224 & 11593615.822 & 11594115.046 \\
\hline 11.190 & 0.850 & -76.300 & 41.460 & 72 & 42 & 289.056 & 138171430.447 & 138171919.501 \\
\hline 11.240 & 1.076 & -76.300 & 41.460 & 72 & 32 & 261.823 & 1381746.242 & 13817828.064 \\
\hline 11.320 & 1.162 & -95.020 & 41.460 & 72 & 41 & 298.986 & 13817930.796 & 138171429.779 \\
\hline 11.320 & 0.405 & -76.300 & 13.050 & 72 & 11 & 299.750 & 138163330.441 & 138163830.191 \\
\hline 11.420 & 0.722 & -76.340 & 41.810 & 72 & 31 & 299.134 & $1381659 \quad .620$ & 13817359.754 \\
\hline 11.460 & 0.756 & -76.130 & 41.680 & 72 & 12 & 299.134 & 138163830.898 & 138164330.029 \\
\hline 11.480 & 0.651 & -76.690 & 13.050 & 72 & 21 & 298.848 & 138164622.829 & 138165121.675 \\
\hline 11.620 & 0.921 & -103.600 & 41.810 & 66 & 32 & 298.883 & 135141910.642 & 13514249.523 \\
\hline 11.650 & 0.805 & -76.780 & 13.050 & 66 & 22 & 299.046 & 13514740.831 & 135141239.875 \\
\hline 11.660 & 0.711 & -96.020 & 41.720 & 66 & 21 & 299.234 & 13514240.759 & 13514739.991 \\
\hline 11.660 & 0.691 & -76.560 & 41.760 & 72 & 22 & 299.146 & 138165122.508 & 138165621.652 \\
\hline 11.700 & 0.884 & -76.740 & 41.550 & 66 & 31 & 299.449 & 135141410.599 & 135141910.046 \\
\hline 11.800 & 0.134 & 11.180 & 12.400 & 67 & 22 & 298.959 & 13516430.791 & 13516929.750 \\
\hline 11.800 & 0.648 & -76.740 & 41.460 & 66 & 12 & 297.491 & 135135345.999 & 135135843.489 \\
\hline 11.820 & 0.484 & -76.690 & 41.630 & 75 & 12 & 299.929 & 206101410.124 & 206101910.051 \\
\hline 11.830 & 0.846 & -76.740 & 41.630 & 66 & 11 & 299.144 & 135134840.309 & 135135339.449 \\
\hline 11.850 & 0.523 & -76.690 & 14.270 & 75 & 11 & 299.439 & 20610910.688 & 206101410.124 \\
\hline 11.880 & 0.183 & 11.230 & 13.050 & 67 & 21 & 299.545 & 135155930.414 & 13516429.957 \\
\hline 11.920 & 0.359 & -76.610 & 12.700 & 73 & 42 & 299.532 & 153121250.225 & 153121749.755 \\
\hline 11.930 & 0.168 & 11.440 & 13.090 & 73 & 41 & 298.510 & 15312750.870 & 153121249.378 \\
\hline 11.930 & 0.160 & 11.400 & 12.570 & 73 & 32 & 273.569 & 15312226.020 & 15312659.586 \\
\hline 11.940 & 0.319 & -76.610 & 12.620 & 67 & 12 & 298.846 & 135155240.559 & 135155739.403 \\
\hline 11.940 & 0.342 & -76.740 & 12.750 & 67 & 31 & 299.288 & 1351611.492 & 135161559.778 \\
\hline 11.940 & 0.242 & 11.230 & 13.050 & 67 & 11 & 299.499 & 135154740.226 & 135155239.723 \\
\hline 11.970 & 1.312 & -76.910 & 48.580 & 71 & 11 & 299.566 & 13815910.495 & 138151410.059 \\
\hline 11.990 & 0.187 & 11.440 & 13.090 & 73 & 31 & 298.996 & 153115720.314 & 15312219.306 \\
\hline 12.000 & 0.350 & -76.740 & 12.750 & 67 & 32 & 299.149 & 1351616.526 & 135162059.672 \\
\hline 12.010 & 0.161 & 11.400 & 12.620 & 70 & 41 & 299.230 & $1352157 \quad .617$ & 13522159.845 \\
\hline 12.020 & 0.308 & -76.650 & 12.830 & 73 & 22 & 292.729 & 153115146.543 & 153115639.272 \\
\hline 12.020 & 0.278 & -76.780 & 12.620 & 70 & 31 & 299.305 & 135214520.167 & 135215019.470 \\
\hline 12.030 & 0.179 & 11.400 & 12.750 & 70 & 32 & 299.151 & 135215020.307 & 135215519.458 \\
\hline 12.030 & 1.198 & -76.910 & 42.070 & 70 & 22 & 254.371 & 135213945.583 & 135214359.952 \\
\hline 12.030 & 0.197 & 11.440 & 12.700 & 70 & 21 & 298.798 & 135213440.919 & 135213939.715 \\
\hline 12.030 & 0.180 & 11.400 & 12.660 & 70 & 42 & 293.820 & 1352225.718 & 13522659.537 \\
\hline 12.050 & 0.377 & -76.740 & 12.700 & 70 & 11 & 299.601 & 135212335.137 & 135212834.737 \\
\hline 12.050 & 0.407 & -76.650 & 13.010 & 68 & 21 & 359.543 & 135171916.509 & 135172516.050 \\
\hline 12.060 & 1.420 & -76.780 & 42.020 & 68 & 22 & 358.295 & 135172522.344 & 135173120.639 \\
\hline 12.060 & 0.282 & 11.050 & 12.880 & 68 & 11 & 299.142 & $13517 \quad 728.499$ & 135171227.639 \\
\hline 12.060 & 0.224 & 11.400 & 12.750 & 70 & 12 & 299.255 & 135212835.569 & 135213334.824 \\
\hline 12.070 & 0.294 & 11.100 & 12.830 & 68 & 12 & 295.419 & 135171233.107 & 135171728.524 \\
\hline 12.080 & 1.111 & -76.820 & 41.940 & 71 & 12 & 299.574 & 138151410.641 & 138151910.211 \\
\hline 12.140 & 0.254 & 11.490 & 12.880 & 73 & 21 & 299.151 & 153114640.756 & 153115139.904 \\
\hline 12.170 & 0.431 & -47.460 & 13.960 & 73 & 12 & 300.008 & 153114050.094 & 153114550.101 \\
\hline 12.180 & 1.509 & -77.040 & 57.010 & 69 & 32 & 293.222 & 135203546.647 & 135204039.869 \\
\hline 12.190 & 1.585 & -77.000 & 65.960 & 69 & 21 & 254.521 & 135201945.149 & 135202359.668 \\
\hline 12.220 & 1.092 & -76.950 & 42.020 & 69 & 31 & 299.679 & 135203040.268 & 135203539.945 \\
\hline 12.230 & 1.764 & -94.460 & 61.270 & 69 & 22 & 224.076 & 135202445.731 & 135202829.803 \\
\hline 12.250 & 0.979 & -77.130 & 42.240 & 71 & 31 & 298.721 & 138153110.531 & 13815369.250 \\
\hline 12.270 & 0.271 & 11.400 & 13.310 & 73 & 11 & 298.742 & 153113550.765 & 153114049.504 \\
\hline 12.290 & 0.506 & -76.690 & 13.400 & 71 & 41 & 299.130 & 138154240.824 & 138154739.951 \\
\hline 12.340 & 0.915 & -77.080 & 42.070 & 71 & 21 & 299.176 & 138152015.474 & 138152514.650 \\
\hline 12.350 & 0.744 & -77.130 & 42.240 & 71 & 32 & 299.142 & 138153610.629 & 13815419.770 \\
\hline 12.480 & 0.789 & -77.040 & 42.020 & 71 & 42 & 287.025 & 138154746.530 & 138155233.554 \\
\hline 12.920 & 0.835 & -49.720 & 14.870 & 75 & 21 & 299.812 & 2061028.205 & $2061033 \quad .013$ \\
\hline 13.400 & 0.559 & -74.820 & 14.440 & 75 & 22 & 289.576 & 20610335.072 & 206103754.646 \\
\hline
\end{tabular}


402: Root Flap Bending Moment, Blade 1 (Instrumented) (N*m)

\begin{tabular}{|c|c|c|c|c|c|c|c|c|}
\hline Mean & Std & Min & $\operatorname{Max}$ & Tape & Event & Time Length & Start Time & End Time \\
\hline-1255.0 & 376.20 & -2115.0 & 427.6 & 67 & 22 & 298.959 & 13516430.791 & 13516929.750 \\
\hline-1134.0 & 358.50 & -2222.0 & 435.5 & 75 & 22 & 289.576 & 20610335.072 & 206103754.646 \\
\hline-1043.0 & 471.10 & -2642.0 & 759.1 & 73 & 42 & 299.532 & 153121250.225 & 153121749.755 \\
\hline-1036.0 & 359.70 & -2264.0 & 2730.0 & 73 & 32 & 273.569 & 15312226.020 & 15312659.586 \\
\hline-881.8 & 406.10 & -2184.0 & 2318.0 & 73 & 41 & 298.510 & 15312750.870 & 153121249.378 \\
\hline-871.9 & 336.10 & -1907.0 & 3037.0 & 65 & 22 & 299.370 & 11594740.501 & 11595239.869 \\
\hline-852.0 & 365.60 & -2090.0 & 3051.0 & 70 & 31 & 299.305 & 135214520.167 & 135215019.470 \\
\hline-809.1 & 321.60 & -2156.0 & 412.5 & 70 & 41 & 299.230 & $1352157 \quad .617$ & 13522159.845 \\
\hline-792.4 & 633.40 & -3229.0 & 3151.0 & 69 & 32 & 293.222 & 135203546.647 & 135204039.869 \\
\hline-786.0 & 458.50 & -1892.0 & 801.1 & 70 & 32 & 299.151 & 135215020.307 & 135215519.458 \\
\hline-727.4 & 407.20 & -3422.0 & 3151.0 & 75 & 21 & 299.812 & $2061028 \quad .205$ & $2061033 \quad .013$ \\
\hline-712.9 & 405.30 & -2556.0 & 1490.0 & 75 & 12 & 299.929 & 206101410.124 & 206101910.051 \\
\hline-656.6 & 453.10 & -2102.0 & 2936.0 & 73 & 31 & 298.996 & 153115720.314 & 15312219.306 \\
\hline-640.0 & 490.40 & -2600.0 & 3153.0 & 69 & 21 & 254.521 & 135201945.149 & 135202359.668 \\
\hline-606.3 & 426.10 & -2555.0 & 3168.0 & 69 & 31 & 299.679 & 135203040.268 & 135203539.945 \\
\hline-579.9 & 372.70 & -2040.0 & 709.2 & 70 & 42 & 293.820 & 1352225.718 & 13522659.537 \\
\hline-538.1 & 382.30 & -1677.0 & 751.7 & 67 & 21 & 299.545 & 135155930.414 & 13516429.957 \\
\hline-511.7 & 335.40 & -1693.0 & 596.3 & 70 & 21 & 298.798 & 135213440.919 & 135213939.715 \\
\hline-507.6 & 449.90 & -3408.0 & 2964.0 & 70 & 22 & 254.371 & 135213945.583 & 135214359.952 \\
\hline-463.4 & 359.10 & -1597.0 & 643.1 & $\overline{70}$ & 11 & 299.601 & 135212335.137 & 135212834.737 \\
\hline-424.8 & 568.50 & -2488.0 & 3127.0 & 69 & 22 & 224.076 & 135202445.731 & 135202829.803 \\
\hline-413.2 & 456.40 & -1890.0 & 2720.0 & 73 & 22 & 292.729 & 153115146.543 & 153115639.272 \\
\hline-401.4 & 366.20 & -3315.0 & 1953.0 & 75 & 11 & 299.439 & 20610910.688 & 206101410.124 \\
\hline-309.6 & 434.10 & -1808.0 & 1046.0 & 70 & 12 & 299.255 & 135212835.569 & 135213334.824 \\
\hline-302.1 & 453.40 & -3407.0 & 3180.0 & 66 & 21 & 299.234 & 13514240.759 & 13514739.991 \\
\hline-252.3 & 375.70 & -1429.0 & 1407.0 & 65 & 21 & 299.355 & 11594240.316 & 11594739.669 \\
\hline-246.0 & 501.10 & -2252.0 & 3224.0 & 72 & 42 & 289.056 & 138171430.447 & 138171919.501 \\
\hline-235.5 & 456.80 & -1684.0 & 1531.0 & 65 & 11 & 299.439 & 11593115.541 & 11593614.980 \\
\hline-220.9 & 448.10 & -2024.0 & 3169.0 & 66 & 22 & 299.046 & 13514740.831 & $13514 \quad 1239.875$ \\
\hline-161.8 & 436.30 & -1715.0 & 1495.0 & 67 & 12 & 298.846 & 135155240.559 & 135155739.403 \\
\hline-152.0 & 471.30 & -2599.0 & 3145.0 & 65 & 12 & 299.224 & 11593615.822 & 11594115.046 \\
\hline-141.1 & 448.80 & -3407.0 & 3175.0 & 66 & 32 & 298.883 & 135141910.642 & 13514249.523 \\
\hline-63.9 & 423.00 & -1512.0 & 1679.0 & 67 & 11 & 299.499 & 135154740.226 & 135155239.723 \\
\hline 15.4 & 757.40 & -3358.0 & 3177.0 & 72 & 32 & 261.823 & 1381746.242 & 13817828.064 \\
\hline 70.1 & 529.30 & -1933.0 & 2438.0 & 67 & 31 & 299.288 & 1351611.492 & 135161559.778 \\
\hline 125.9 & 502.00 & -2429.0 & 3169.0 & 66 & 31 & 299.449 & 135141410.599 & 135141910.046 \\
\hline 193.0 & 411.90 & -1250.0 & 1652.0 & 73 & 21 & 299.151 & 153114640.756 & 153115139.904 \\
\hline 255.5 & 515.40 & -2460.0 & 2402.0 & 73 & 12 & 300.008 & 153114050.094 & 153114550.101 \\
\hline 290.7 & 468.90 & -1422.0 & 2053.0 & 67 & 32 & 299.149 & $1351616 \quad .526$ & 135162059.672 \\
\hline 296.4 & 473.80 & -2856.0 & 3193.0 & 66 & 12 & 297.491 & 135135345.999 & 135135843.489 \\
\hline 322.7 & 770.60 & -1999.0 & 3237.0 & 72 & 41 & 298.986 & 13817930.796 & 138171429.779 \\
\hline 331.0 & 795.70 & -2130.0 & 3237.0 & 72 & 11 & 299.750 & 138163330.441 & 138163830.191 \\
\hline 610.3 & 542.80 & -1045.0 & 2926.0 & 73 & 11 & 298.742 & 153113550.765 & 153114049.504 \\
\hline 639.7 & 613.30 & -2254.0 & 3237.0 & 72 & 31 & 299.134 & $1381659 \quad .620$ & 13817359.754 \\
\hline $672 . \overline{7}$ & 592.80 & -2291.0 & 3278.0 & $\overline{71}$ & 11 & 299.566 & 13815910.495 & 138151410.059 \\
\hline 685.3 & 532.80 & -993.8 & 2992.0 & 68 & 11 & 299.142 & 13517728.499 & 135171227.639 \\
\hline 697.2 & 654.30 & -2315.0 & 3278.0 & 71 & 12 & 299.574 & 138151410.641 & 138151910.211 \\
\hline 705.7 & 503.50 & -2674.0 & 3040.0 & 66 & 11 & 299.144 & 135134840.309 & 135135339.449 \\
\hline 712.6 & 628.00 & -1629.0 & 3126.0 & 68 & 21 & 359.543 & $13517 \quad 1916.509$ & 135172516.050 \\
\hline 722.3 & 683.60 & -2177.0 & 3126.0 & 68 & 12 & 295.419 & $135 \quad 17 \quad 1233.107$ & $135 \quad 17 \quad 1728.524$ \\
\hline 745.8 & 730.00 & -2423.0 & 3237.0 & 72 & 21 & 298.848 & 138164622.829 & 138165121.675 \\
\hline 799.6 & 585.10 & -3393.0 & 3126.0 & 68 & 22 & 358.295 & 135172522.344 & 13517.3120 .639 \\
\hline 809.3 & 683.70 & -1806.0 & 3237.0 & 72 & 12 & 299.134 & 138163830.898 & 138164330.029 \\
\hline 1014.0 & 699.10 & -2727.0 & 3278.0 & 71 & 31 & 298.721 & 138153110.531 & 13815369.250 \\
\hline 1071.0 & 628.10 & -2098.0 & 3278.0 & 71 & 41 & 299.130 & 138154240.824 & 138154739.951 \\
\hline 1177.0 & 688.60 & -2465.0 & 3278.0 & $\overline{71}$ & 32 & 299.142 & 138153610.629 & 13815419.770 \\
\hline 1205.0 & 770.90 & -2544.0 & 3237.0 & 72 & 22 & 299.146 & 138165122.508 & 138165621.652 \\
\hline 1240.0 & 794.40 & -2725.0 & 3278.0 & 71 & 21 & 299.176 & $138 \quad 152015.474$ & 138152514.650 \\
\hline 1457.0 & 700.40 & -2178.0 & 3278.0 & 71 & 42 & 287.025 & 138154746.530 & 138155233.554 \\
\hline
\end{tabular}


403: Root Flap Bending Moment, Blade $2\left(N^{*} m\right)$

\begin{tabular}{|c|c|c|c|c|c|c|c|c|}
\hline Mean & Std & Min & Max & Tape & Event & Time Length & Start Time & End Time \\
\hline-1365.0 & 375.00 & -2221.0 & 226.1 & 67 & 22 & 298.959 & 13516430.791 & 13516929.750 \\
\hline-1191.0 & 469.20 & -2550.0 & 1657.0 & 73 & 42 & 299.532 & 153121250.225 & 153121749.755 \\
\hline-1182.0 & 357.30 & -2352.0 & 1817.0 & 73 & 32 & 273.569 & 15312226.020 & 15312659.586 \\
\hline-1104.0 & 346.80 & -2159.0 & 2145.0 & 75 & 22 & 289.576 & 20610335.072 & 206103754.646 \\
\hline-1030.0 & 404.30 & -2318.0 & 2088.0 & 73 & 41 & 298.510 & 15312750.870 & 153121249.378 \\
\hline-939.8 & 362.80 & -2095.0 & 188.4 & 70 & 31 & 299.305 & 135214520.167 & 135215019.470 \\
\hline-904.7 & 333.20 & -1686.0 & 2122.0 & 65 & 22 & 299.370 & 11594740.501 & 11595239.869 \\
\hline-895.9 & 313.90 & -2175.0 & 573.7 & 70 & 41 & 299.230 & $\begin{array}{lll}1352157 & .617\end{array}$ & 13522159.845 \\
\hline-875.0 & 451.80 & -2047.0 & 890.1 & 70 & 32 & 299.151 & 135215020.307 & 135215519.458 \\
\hline-837.0 & 402.40 & -2050.0 & 2007.0 & 75 & 12 & 299.929 & 206101410.124 & 206101910.051 \\
\hline-795.0 & 624.60 & -2435.0 & 2335.0 & 69 & 32 & 293.222 & 135203546.647 & 135204039.869 \\
\hline-794.8 & 449.50 & -2386.0 & 1857.0 & $\overline{73}$ & 31 & 298.996 & 153115720.314 & 15312219.306 \\
\hline-696.5 & 376.80 & -2305.0 & 2034.0 & 75 & 21 & 299.812 & $2061028 \quad .205$ & $2061033 \quad .013$ \\
\hline-668.2 & 362.30 & -2183.0 & 566.6 & 70 & 42 & 293.820 & 1352225.718 & 13522659.537 \\
\hline-636.0 & 367.60 & -1894.0 & 627.0 & 67 & 21 & 299.545 & 135155930.414 & $13516 \quad 429.957$ \\
\hline-635.7 & 476.70 & -2435.0 & 2428.0 & 69 & 21 & 254.521 & 135201945.149 & 135202359.668 \\
\hline-598.8 & 415.20 & -2435.0 & 2415.0 & 69 & 31 & 299.679 & 135203040.268 & 135203539.945 \\
\hline-597.3 & 323.70 & -1902.0 & 873.5 & 70 & 21 & 298.798 & 135213440.919 & 135213939.715 \\
\hline-594.0 & 438.40 & -2498.0 & 2194.0 & 70 & 22 & 254.371 & 135213945.583 & 135214359.952 \\
\hline-552.8 & 346.30 & -1770.0 & 529.7 & 70 & 11 & 299.601 & 135212335.137 & 135212834.737 \\
\hline-550.9 & 445.00 & -1973.0 & 1058.0 & 73 & 22 & 292.729 & 153115146.543 & 153115639.272 \\
\hline-527.8 & 354.40 & -1880.0 & 2164.0 & 75 & 11 & 299.439 & 20610910.688 & 206101410.124 \\
\hline-424.7 & 557.10 & -2073.0 & 2348.0 & 69 & 22 & 224.076 & 135202445.731 & 135202829.803 \\
\hline-401.8 & 424.80 & -1944.0 & 1066.0 & 70 & 12 & 299.255 & 135212835.569 & 135213334.824 \\
\hline-350.0 & 489.50 & -1807.0 & 2316.0 & 72 & 42 & 289.056 & 138171430.447 & 138171919.501 \\
\hline-340.4 & 442.80 & -2467.0 & 2059.0 & 66 & 21 & 299.234 & 13514240.759 & 13514739.991 \\
\hline-289.9 & 349.10 & -1485.0 & 1281.0 & 65 & 21 & 299.355 & 11594240.316 & 11594739.669 \\
\hline-289.9 & 435.10 & -1758.0 & 2009.0 & 65 & 11 & 299.439 & 11593115.541 & 11593614.980 \\
\hline-262.1 & 416.60 & -1782.0 & 1957.0 & 67 & 12 & 298.846 & 135155240.559 & 135155739.403 \\
\hline-262.0 & 431.50 & -1693.0 & 2379.0 & 66 & 22 & 299.046 & 13514740.831 & 135141239.875 \\
\hline-198.6 & 445.30 & -1767.0 & 2160.0 & 65 & 12 & 299.224 & 11593615.822 & 11594115.046 \\
\hline-187.9 & 425.00 & -2394.0 & 2149.0 & 66 & 32 & 298.883 & 135141910.642 & 13514249.523 \\
\hline-170.0 & 398.70 & -1583.0 & 1970.0 & 67 & 11 & 299.499 & 135154740.226 & 135155239.723 \\
\hline-100.1 & 731.70 & -2470.0 & 2401.0 & 72 & 32 & 261.823 & 1381746.242 & 13817828.064 \\
\hline-39.7 & 514.50 & -2104.0 & 2012.0 & 67 & 31 & 299.288 & $1351611 \quad .492$ & 135161559.778 \\
\hline 43.0 & 385.20 & -1604.0 & 1541.0 & 73 & 21 & 299.151 & 153114640.756 & 153115139.904 \\
\hline 71.2 & 475.10 & -1762.0 & 2360.0 & 66 & 31 & 299.449 & 135141410.599 & 135141910.046 \\
\hline 99.6 & 487.70 & -1789.0 & 2001.0 & 73 & 12 & 300.008 & 153114050.094 & 153114550.101 \\
\hline 173.0 & 442.20 & -1575.0 & 1839.0 & 67 & 32 & 299.149 & 1351616.526 & 135162059.672 \\
\hline 184.8 & 762.00 & -2406.0 & 2401.0 & 72 & 11 & 299.750 & 138163330.441 & 138163830.191 \\
\hline 185.9 & 710.90 & -1832.0 & 2401.0 & 72 & 41 & 298.986 & 13817930.796 & 138171429.779 \\
\hline 246.3 & 450.10 & -1975.0 & 2370.0 & 66 & 12 & 297.491 & 135135345.999 & 135135843.489 \\
\hline 422.0 & 505.70 & -1193.0 & 2321.0 & 73 & 11 & 298.742 & 153113550.765 & 153114049.504 \\
\hline 496.7 & 589.50 & -1612.0 & 2401.0 & 72 & 31 & 299.134 & $1381659 \quad .620$ & 13817359.754 \\
\hline 514.1 & 551.80 & -2215.0 & 2423.0 & 71 & 11 & 299.566 & 13815910.495 & 138151410.059 \\
\hline 531.9 & 616.60 & -2368.0 & 2423.0 & 71 & 12 & 299.574 & 138151410.641 & 138151910.211 \\
\hline 585.8 & 685.40 & -2021.0 & 2401.0 & 72 & 21 & 298.848 & 138164622.829 & 138165121.675 \\
\hline 639.1 & 476.50 & -1557.0 & 2404.0 & 66 & 11 & 299.144 & 135134840.309 & 135135339.449 \\
\hline 647.1 & 630.90 & -1637.0 & 2401.0 & 72 & 12 & 299.134 & 138163830.898 & 138164330.029 \\
\hline 664.2 & 494.40 & -913.5 & 2457.0 & 68 & 11 & 299.142 & 13517728.499 & 135171227.639 \\
\hline 697.4 & 600.80 & -1440.0 & 2457.0 & 68 & 21 & 359.543 & 135171916.509 & 135172516.050 \\
\hline 700.6 & 644.50 & -2364.0 & 2457.0 & 68 & 12 & 295.419 & 135171233.107 & 135171728.524 \\
\hline 773.1 & 548.00 & -1894.0 & 2457.0 & 68 & 22 & 358.295 & 135172522.344 & 135173120.639 \\
\hline 803.6 & 658.00 & -2279.0 & 2423.0 & 71 & 31 & 298.721 & 138153110.531 & 13815369.250 \\
\hline 851.4 & 598.40 & -1653.0 & 2423.0 & 71 & 41 & 299.130 & 138154240.824 & 138154739.951 \\
\hline 946.3 & 650.40 & -2448.0 & 2423.0 & 71 & 32 & 299.142 & 138153610.629 & 13815419.770 \\
\hline 999.9 & 722.10 & -2025.0 & 2401.0 & 72 & 22 & 299.146 & 138165122.508 & 138165621.652 \\
\hline 1002.0 & 741.50 & -2159.0 & 2423.0 & 71 & 21 & 299.176 & 138152015.474 & 138152514.650 \\
\hline 1186.0 & 657.70 & -1964.0 & 2423.0 & 71 & 42 & 287.025 & 138154746.530 & 138155233.554 \\
\hline
\end{tabular}


404: Root Flap Bending Moment, Blade $3\left(\mathrm{~N}^{*} \mathrm{~m}\right)$

\begin{tabular}{|c|c|c|c|c|c|c|c|c|}
\hline Mean & Std & Min & $\operatorname{Max}$ & Tape & Event & Time Length & Start Time & End Time \\
\hline-1217.0 & 387.60 & -2086.0 & 236.7 & 67 & 22 & 298.959 & $13516 \quad 430.791$ & 13516929.750 \\
\hline-1072.0 & 478.80 & -2388.0 & 801.4 & 73 & $\overline{42}$ & 299.532 & 153121250.225 & 153121749.755 \\
\hline-1058.0 & 367.60 & -2275.0 & 1514.0 & 73 & 32 & 273.569 & 15312226.020 & 15312659.586 \\
\hline-1028.0 & 365.60 & -2057.0 & 1809.0 & 75 & 22 & 289.576 & 20610335.072 & 206103754.646 \\
\hline-903.8 & 414.60 & -2388.0 & 1636.0 & 73 & 41 & 298.510 & 15312750.870 & 153121249.378 \\
\hline-862.0 & 631.80 & -2460.0 & 2017.0 & 69 & 32 & 293.222 & 135203546.647 & 135204039.869 \\
\hline-831.9 & 370.60 & -1946.0 & 212.6 & 70 & 31 & 299.305 & 135214520.167 & 135215019.470 \\
\hline-793.7 & 341.20 & -1572.0 & 2071.0 & 65 & 22 & 299.370 & 11594740.501 & 11595239.869 \\
\hline-789.2 & 326.50 & -2130.0 & 664.9 & 70 & 41 & 299.230 & $1352157 \quad .617$ & 13522159.845 \\
\hline-767.0 & 460.80 & -1973.0 & 908.7 & 70 & 32 & 299.151 & 135215020.307 & 135215519.458 \\
\hline-707.7 & 479.80 & -2460.0 & 2015.0 & 69 & 21 & 254.521 & 135201945.149 & 135202359.668 \\
\hline-673.2 & 453.40 & -2041.0 & 1730.0 & 73 & 31 & 298.996 & 153115720.314 & 15312219.306 \\
\hline-668.0 & 426.20 & -2460.0 & 1958.0 & 69 & 31 & 299.679 & 135203040.268 & 135203539.945 \\
\hline-621.3 & 405.20 & -2211.0 & 2107.0 & 75 & 12 & 299.929 & 206101410.124 & 206101910.051 \\
\hline-618.6 & 402.10 & -2067.0 & 1926.0 & 75 & 21 & 299.812 & 2061028.205 & $2061033 \quad .013$ \\
\hline-563.1 & 367.80 & -1984.0 & 1572.0 & 70 & $\overline{42}$ & 293.820 & 1352225.718 & 13522659.537 \\
\hline-495.3 & 331.80 & -1626.0 & 716.6 & 70 & 21 & 298.798 & 135213440.919 & 135213939.715 \\
\hline-491.8 & 562.90 & -2235.0 & 2023.0 & 69 & 22 & 224.076 & 135202445.731 & 135202829.803 \\
\hline-488.7 & 440.90 & -1790.0 & 2120.0 & 70 & 22 & 254.371 & 135213945.583 & 135214359.952 \\
\hline-485.0 & 378.70 & -1664.0 & 753.9 & 67 & 21 & 299.545 & 135155930.414 & 13516429.957 \\
\hline-454.2 & 352.60 & -1634.0 & 739.6 & 70 & 11 & 299.601 & 135212335.137 & 135212834.737 \\
\hline-430.1 & 449.00 & -1746.0 & 1904.0 & 73 & 22 & 292.729 & 153115146.543 & 153115639.272 \\
\hline-319.5 & 356.40 & -2256.0 & 2146.0 & 75 & 11 & 299.439 & 20610910.688 & 206101410.124 \\
\hline-317.1 & 490.30 & -1926.0 & 2093.0 & 72 & 42 & 289.056 & 138171430.447 & 138171919.501 \\
\hline-307.9 & 444.00 & -2300.0 & 2080.0 & 66 & 21 & 299.234 & 13514240.759 & 13514739.991 \\
\hline-301.9 & 429.90 & -1840.0 & 1729.0 & 70 & 12 & 299.255 & 135212835.569 & 135213334.824 \\
\hline-226.8 & 434.50 & -1936.0 & 2088.0 & 66 & 22 & 299.046 & 13514740.831 & 135141239.875 \\
\hline-186.4 & 351.90 & -1320.0 & 1691.0 & 65 & 21 & 299.355 & 11594240.316 & 11594739.669 \\
\hline-182.3 & 435.40 & -1706.0 & 1291.0 & 65 & 11 & 299.439 & 11593115.541 & 11593614.980 \\
\hline-158.9 & 428.80 & -1644.0 & 2097.0 & 66 & 32 & 298.883 & 135141910.642 & 13514249.523 \\
\hline-117.0 & 422.70 & -1712.0 & 1512.0 & 67 & 12 & 298.846 & 135155240.559 & 135155739.403 \\
\hline-95.7 & 445.90 & -1724.0 & 2093.0 & 65 & 12 & 299.224 & 11593615.822 & 11594115.046 \\
\hline-71.6 & 729.00 & -2401.0 & 2095.0 & 72 & 32 & 261.823 & 1381746.242 & 13817828.064 \\
\hline-27.2 & 403.90 & -1286.0 & 1531.0 & 67 & 11 & 299.499 & 135154740.226 & 135155239.723 \\
\hline 97.9 & 515.10 & -2244.0 & 2158.0 & 67 & 31 & 299.288 & $\begin{array}{lll}1351611 & .492 \\
\end{array}$ & 135161559.778 \\
\hline 100.2 & 478.20 & -1958.0 & 2061.0 & 66 & 31 & 299.449 & 135141410.599 & 135141910.046 \\
\hline 159.1 & 396.20 & -1305.0 & 1743.0 & 73 & 21 & 299.151 & 153114640.756 & 153115139.904 \\
\hline 210.3 & 709.90 & -2169.0 & 2095.0 & 72 & 41 & 298.986 & 13817930.796 & 138171429.779 \\
\hline 213.6 & 489.00 & -2242.0 & 2108.0 & 73 & 12 & 300.008 & 153114050.094 & 153114550.101 \\
\hline 221.9 & 756.60 & -2353.0 & 2095.0 & 72 & 11 & 299.750 & 138163330.441 & 138163830.191 \\
\hline 266.1 & 461.80 & -1638.0 & 2009.0 & 66 & 12 & 297.491 & 135135345.999 & 135135843.489 \\
\hline 303.6 & 450.30 & -1208.0 & 2158.0 & 67 & 32 & 299.149 & $1351616 \quad .526$ & 135162059.672 \\
\hline 512.8 & 578.50 & -1605.0 & 2095.0 & 72 & 31 & 299.134 & 1381659.620 & 13817359.754 \\
\hline 535.9 & 508.80 & -1318.0 & 2108.0 & 73 & 11 & 298.742 & 153113550.765 & 153114049.504 \\
\hline 539.7 & 549.80 & -1922.0 & 2120.0 & 71 & 11 & 299.566 & 13815910.495 & 138151410.059 \\
\hline 562.9 & 615.90 & -2068.0 & 2120.0 & 71 & 12 & 299.574 & 138151410.641 & $1381519 \quad 10.211$ \\
\hline 605.2 & 667.10 & -2011.0 & 2095.0 & 72 & 21 & 298.848 & 138164622.829 & 138165121.675 \\
\hline 635.0 & 470.80 & -1564.0 & 2099.0 & 66 & 11 & 299.144 & 135134840.309 & 135135339.449 \\
\hline 663.6 & 620.00 & -1702.0 & 2095.0 & 72 & 12 & 299.134 & 138163830.898 & 138164330.029 \\
\hline 731.8 & 492.20 & -935.2 & 2188.0 & 68 & 11 & 299.142 & 13517728.499 & 135171227.639 \\
\hline 757.8 & 598.50 & -1663.0 & 2188.0 & 68 & 21 & 359.543 & 135171916.509 & 135172516.050 \\
\hline 768.2 & 646.60 & -2079.0 & 2188.0 & 68 & 12 & 295.419 & 135171233.107 & 135171728.524 \\
\hline 833.8 & 640.90 & -2377.0 & 2120.0 & 71 & 31 & 298.721 & $1381531 \quad 10.531$ & 13815369.250 \\
\hline 835.9 & 549.60 & -2042.0 & 2188.0 & 68 & 22 & 358.295 & 135172522.344 & 135173120.639 \\
\hline 881.6 & 583.90 & -2242.0 & 2120.0 & 71 & 41 & 299.130 & 138154240.824 & 138154739.951 \\
\hline 989.7 & 628.10 & -1823.0 & 2120.0 & 71 & 32 & 299.142 & 138153610.629 & 13815419.770 \\
\hline 1017.0 & 697.30 & -1986.0 & 2095.0 & 72 & 22 & 299.146 & 138165122.508 & 138165621.652 \\
\hline 1040.0 & 714.70 & -2377.0 & 2120.0 & 71 & 21 & 299.176 & 138152015.474 & 138152514.650 \\
\hline 1226.0 & 610.00 & -1733.0 & 2120.0 & 71 & 42 & 287.025 & 138154746.530 & 138155233.554 \\
\hline
\end{tabular}


414: Root Edge Bending Moment $\left(\mathrm{N}^{*} \mathrm{~m}\right)$

\begin{tabular}{|c|c|c|c|c|c|c|c|c|}
\hline Mean & Std̃ & Min & Max & Tape & Event & Time Length & Start Time & End Time \\
\hline 158.8 & 697.0 & -1341 & 3027 & 65 & 22 & 299.370 & 11594740.501 & 11595239.869 \\
\hline 189.8 & 698.9 & -2111 & 2933 & 75 & 12 & 299.929 & 206101410.124 & 206101910.051 \\
\hline 197.7 & 690.7 & -2218 & 2698 & 75 & 22 & 289.576 & 20610335.072 & 206103754.646 \\
\hline 198.7 & 691.6 & -1685 & 3154 & 73 & 32 & 273.569 & 15312226.020 & 15312659.586 \\
\hline 204.8 & 694.5 & -1130 & 1608 & 73 & 41 & 298.510 & 15312750.870 & 153121249.378 \\
\hline 207.6 & 692.3 & -1061 & 1868 & 73 & 42 & 299.532 & 153121250.225 & 153121749.755 \\
\hline 209.5 & 709.4 & -2335 & 3053 & 66 & 21 & 299.234 & 13514240.759 & 13514739.991 \\
\hline 210.5 & 688.1 & -973 & 1492 & 67 & 22 & 298.959 & 13516430.791 & 13516929.750 \\
\hline 212.0 & 699.2 & -2221 & 2182 & 75 & 21 & 299.812 & 2061028.205 & $2061033 \quad .013$ \\
\hline 219.3 & 690.6 & -1004 & 1640 & 70 & 41 & 299.230 & $1352157 \quad .617$ & 13522159.845 \\
\hline 221.4 & 691.3 & -1001 & 1550 & 70 & 31 & 299.305 & 135214520.167 & 135215019.470 \\
\hline 222.3 & 700.9 & -1881 & 2650 & 73 & 31 & 298.996 & 153115720.314 & 15312219.306 \\
\hline 222.6 & 708.3 & -3159 & 3016 & 75 & 11 & 299.439 & 20610910.688 & 206101410.124 \\
\hline 222.8 & 714.1 & -1764 & 2924 & 66 & 22 & 299.046 & 13514740.831 & 135141239.875 \\
\hline 226.8 & 710.2 & -1162 & 2750 & 65 & 11 & 299.439 & 11593115.541 & 11593614.980 \\
\hline 227.4 & 709.2 & -1206 & 1836 & 65 & 21 & 299.355 & 11594240.316 & 11594739.669 \\
\hline 229.3 & 694.4 & -1097 & 1840 & 70 & 32 & 299.151 & 135215020.307 & 135215519.458 \\
\hline 235.2 & 714.2 & -2187 & 3144 & 66 & 32 & 298.883 & 135141910.642 & 13514249.523 \\
\hline 239.9 & 701.5 & -1074 & 1786 & 67 & 21 & 299.545 & 135155930.414 & 13516429.957 \\
\hline 240.8 & 698.2 & -1105 & 1780 & 70 & 42 & 293.820 & 1352225.718 & 13522659.537 \\
\hline 243.9 & 712.9 & -2190 & 3145 & 72 & 42 & 289.056 & 138171430.447 & 138171919.501 \\
\hline 245.9 & 698.3 & -1109 & 1796 & 70 & 21 & 298.798 & 135213440.919 & 135213939.715 \\
\hline 247.7 & 713.9 & -2307 & 3203 & 65 & 12 & 299.224 & 11593615.822 & 11594115.046 \\
\hline 249.2 & 706.5 & -1234 & 1970 & 73 & 22 & 292.729 & 153115146.543 & 153115639.272 \\
\hline 252.5 & 703.7 & -2355 & 2756 & 70 & 22 & 254.371 & 135213945.583 & 135214359.952 \\
\hline 253.7 & 701.9 & -1097 & 1788 & 70 & 11 & 299.601 & 135212335.137 & 135212834.737 \\
\hline 254.5 & 700.9 & -2405 & 2813 & 69 & 21 & 254.521 & 135201945.149 & 135202359.668 \\
\hline 254.9 & 702.3 & -3208 & 3166 & 69 & 31 & 299.679 & 135203040.268 & 135203539.945 \\
\hline 259.8 & 703.2 & -2249 & 2810 & 69 & 32 & 293.222 & 135203546.647 & 135204039.869 \\
\hline 275.4 & 707.6 & -1114 & 1843 & 70 & 12 & 299.255 & 135212835.569 & 135213334.824 \\
\hline 279.2 & 710.8 & -2772 & 3092 & 69 & 22 & 224.076 & 135202445.731 & 135202829.803 \\
\hline 280.1 & 720.3 & -2052 & 3041 & 66 & 31 & 299.449 & 135141410.599 & 135141910.046 \\
\hline 291.3 & 716.6 & -1473 & 2035 & 67 & 12 & 298.846 & 135155240.559 & 135155739.403 \\
\hline 294.1 & 734.7 & -2058 & 3164 & 72 & 32 & 261.823 & 1381746.242 & 13817828.064 \\
\hline 306.2 & 719.4 & -1204 & 2085 & 67 & 11 & 299.499 & 135154740.226 & 135155239.723 \\
\hline 312.2 & 754.0 & -2597 & 3061 & 71 & 12 & 299.574 & 138151410.641 & 138151910.211 \\
\hline 312.5 & 725.3 & -2175 & 3085 & 66 & 12 & 297.491 & 135135345.999 & 135135843.489 \\
\hline 313.5 & 753.5 & -2534 & 3092 & 71 & 11 & 299.566 & 13815910.495 & 138151410.059 \\
\hline 331.3 & 728.5 & -1393 & 2052 & 67 & 31 & 299.288 & 1351611.492 & 135161559.778 \\
\hline 331.8 & 743.2 & -2365 & 3107 & 72 & 41 & 298.986 & 13817930.796 & 138171429.779 \\
\hline 340.9 & 724.2 & -2236 & 2175 & 73 & 21 & 299.151 & 153114640.756 & 153115139.904 \\
\hline 345.4 & 751.1 & -2128 & 3176 & 72 & 11 & 299.750 & 138163330.441 & 138163830.191 \\
\hline 354.8 & 726.3 & -2340 & 2424 & 73 & 12 & 300.008 & 153114050.094 & 153114550.101 \\
\hline 359.5 & 767.2 & -2142 & 2913 & 71 & 31 & 298.721 & 138153110.531 & 13815369.250 \\
\hline 364.5 & 789.6 & -2887 & 3092 & 71 & 21 & 299.176 & 138152015.474 & 138152514.650 \\
\hline 364.6 & 732.9 & -1315 & 2502 & 68 & 11 & 299.142 & 13517728.499 & 135171227.639 \\
\hline 364.9 & 772.3 & -2062 & 3078 & 71 & 32 & 299.142 & 138153610.629 & 13815419.770 \\
\hline 365.2 & 752.2 & -1680 & 2666 & 68 & 12 & 295.419 & 135171233.107 & 135171728.524 \\
\hline 367.3 & 753.0 & -1768 & 2597 & 68 & 21 & 359.543 & 135171916.509 & 135172516.050 \\
\hline 369.5 & 729.9 & -1321 & 2137 & 67 & 32 & 299.149 & $1351616 \quad .526$ & 135162059.672 \\
\hline 371.1 & 772.0 & -2005 & 3045 & 71 & 41 & 299.130 & 138154240.824 & 138154739.951 \\
\hline 383.4 & 751.8 & -2861 & 3093 & 68 & 22 & 358.295 & 135172522.344 & 135173120.639 \\
\hline 383.5 & 793.9 & -2003 & 3092 & 71 & 42 & 287.025 & 138154746.530 & 138155233.554 \\
\hline 387.5 & 730.1 & -1899 & 3100 & 66 & 11 & 299.144 & 135134840.309 & 135135339.449 \\
\hline 392.7 & 739.0 & -2179 & 3118 & 72 & 31 & 299.134 & $1381659 \quad .620$ & 13817359.754 \\
\hline 399.9 & 745.9 & -2593 & 2662 & 72 & 21 & 298.848 & 138164622.829 & 138165121.675 \\
\hline 407.3 & $75 \overline{7.0}$ & -2193 & 2972 & 72 & 12 & 299.134 & 138163830.898 & 138164330.029 \\
\hline 416.9 & 734.2 & -1667 & 2965 & 73 & 11 & 298.742 & 153113550.765 & 153114049.504 \\
\hline 443.8 & 796.0 & -2135 & 3126 & 72 & 22 & 299.146 & 138165122.508 & 138165621.652 \\
\hline
\end{tabular}


423: Low Speed Shaft Torque $\left(\mathrm{N}^{*} \mathrm{~m}\right)$

\begin{tabular}{|c|c|c|c|c|c|c|c|c|}
\hline Mean & Std & Min & $\operatorname{Max}$ & Tape & Event & Time Length & Start Time & End Time \\
\hline-277.6 & 235.8 & -2454 & 739 & $\overline{67}$ & 22 & 298.959 & 13516430.791 & 13516929.750 \\
\hline-228.5 & 223.6 & -3202 & 5925 & 73 & 32 & 273.569 & 15312226.020 & 15312659.586 \\
\hline-209.1 & 324.5 & -1441 & 4559 & 73 & 42 & 299.532 & 153121250.225 & 153121749.755 \\
\hline-189.3 & 237.2 & -2399 & 5769 & 75 & 22 & 289.576 & 20610335.072 & 206103754.646 \\
\hline-102.1 & 263.8 & -1219 & 5709 & $\overline{73}$ & 41 & 298.510 & 15312750.870 & 153121249.378 \\
\hline-100.0 & 226.3 & -3543 & 5895 & 65 & 22 & 299.370 & 11594740.501 & 11595239.869 \\
\hline 7.3 & 258.3 & -615 & 5747 & 70 & 31 & 299.305 & 135214520.167 & 135215019.470 \\
\hline 31.7 & 192.6 & -361 & 829 & 70 & $\overline{41}$ & 299.230 & $1352157 \quad .617$ & 13522159.845 \\
\hline 78.0 & 357.5 & -483 & 5321 & 70 & 32 & 299.151 & 135215020.307 & 135215519.458 \\
\hline 117.2 & 357.5 & -3759 & 2957 & 73 & 31 & 298.996 & 153115720.314 & 15312219.306 \\
\hline 132.0 & 535.9 & -3775 & 5906 & 69 & 32 & 293.222 & 135203546.647 & 135204039.869 \\
\hline 141.5 & 320.2 & -3645 & 5236 & 75 & 12 & 299.929 & 206101410.124 & 206101910.051 \\
\hline 193.9 & 307.2 & -4358 & 4038 & 75 & 21 & 299.812 & $2061028 \quad .205$ & 2061033.013 \\
\hline 233.0 & 405.8 & -4071 & 5996 & 69 & 21 & 254.521 & 135201945.149 & 135202359.668 \\
\hline 257.3 & 343.2 & -6258 & 5948 & 69 & 31 & 299.679 & 135203040.268 & 135203539.945 \\
\hline 261.6 & 256.1 & -474 & 2752 & 70 & 42 & 293.820 & 1352225.718 & 13522659.537 \\
\hline 321.4 & 294.2 & -336 & 2458 & 67 & 21 & 299.545 & 135155930.414 & 13516429.957 \\
\hline 328.1 & 205.7 & -247 & 2895 & 70 & 21 & 298.798 & 135213440.919 & 135213939.715 \\
\hline 352.7 & 370.9 & -3934 & 3554 & 70 & 22 & 254.371 & 135213945.583 & 135214359.952 \\
\hline 372.5 & 405.9 & -395 & 2756 & 73 & 22 & 292.729 & 153115146.543 & 153115639.272 \\
\hline 383.1 & 258.8 & -253 & 2847 & 70 & 11 & 299.601 & 135212335.137 & 135212834.737 \\
\hline 438.1 & 373.1 & -290 & 2726 & 65 & 11 & 299.439 & 11593115.541 & 11593614.980 \\
\hline 438.8 & 290.7 & -188 & 2840 & 65 & 21 & 299.355 & 11594240.316 & 11594739.669 \\
\hline 459.2 & 283.9 & -4867 & 5895 & 75 & 11 & 299.439 & 20610910.688 & 206101410.124 \\
\hline 471.7 & 507.4 & -5551 & 5888 & 69 & 22 & 224.076 & 135202445.731 & 135202829.803 \\
\hline 488.9 & 328.4 & -5784 & 4519 & 66 & 21 & 299.234 & 13514240.759 & 13514739.991 \\
\hline 546.7 & 401.6 & -4067 & 3655 & 65 & 12 & 299.224 & 11593615.822 & 11594115.046 \\
\hline 550.4 & 434.2 & -4032 & 5963 & $\overline{72}$ & 42 & 289.056 & 138171430.447 & 138171919.501 \\
\hline 561.6 & 325.6 & -370 & 2943 & 70 & 12 & 299.255 & 135212835.569 & 135213334.824 \\
\hline 586.3 & 361.9 & -3763 & 2857 & 66 & 22 & 299.046 & 13514740.831 & 135141239.875 \\
\hline 672.5 & 398.1 & -5710 & 3516 & 66 & 32 & 298.883 & 135141910.642 & 13514249.523 \\
\hline 747.2 & 325.3 & -52 & 2578 & 67 & 12 & 298.846 & 135155240.559 & 135155739.403 \\
\hline 859.1 & 336.7 & -31 & 2797 & 67 & 11 & 299.499 & 135154740.226 & 135155239.723 \\
\hline 869.1 & 694.8 & -4098 & 3507 & 72 & 32 & 261.823 & $138174 \cdot 6.242$ & 13817828.064 \\
\hline 996.4 & 461.6 & -4020 & 3765 & 66 & 31 & 299.449 & 135141410.599 & 135141910.046 \\
\hline 1030.0 & 417.6 & -330 & 2734 & 67 & 31 & 299.288 & $1351611 \quad .492$ & 135161559.778 \\
\hline 1086.0 & 318.9 & 75 & 2007 & 73 & 21 & 299.151 & 153114640.756 & 153115139.904 \\
\hline 1167.0 & 459.0 & -3843 & 2366 & 73 & 12 & 300.008 & 153114050.094 & 153114550.101 \\
\hline 1174.0 & 715.4 & -5530 & 4103 & 72 & 41 & 298.986 & 13817930.796 & 138171429.779 \\
\hline 1224.0 & 724.8 & -501 & 5981 & 72 & 11 & 299.750 & 138163330.441 & 138163830.191 \\
\hline 1232.0 & 388.9 & -4140 & 3417 & 66 & 12 & 297.491 & 135135345.999 & 135135843.489 \\
\hline 1302.0 & 366.9 & 275 & 2749 & 67 & 32 & 299.149 & $1351616 \quad .526$ & 135162059.672 \\
\hline 1570.0 & 502.5 & -3742 & 3899 & 72 & 31 & 299.134 & 1381659.620 & 13817359.754 \\
\hline 1583.0 & 450.5 & 108 & 2624 & 73 & 11 & 298.742 & 153113550.765 & 153114049.504 \\
\hline 1595.0 & 452.0 & -4417 & 3943 & 71 & 11 & 299.566 & 13815910.495 & 138151410.059 \\
\hline 1620.0 & 505.0 & -4423 & 3898 & 71 & 12 & 299.574 & 138151410.641 & 138151910.211 \\
\hline 1642.0 & 596.8 & -4392 & 2914 & 72 & 21 & 298.848 & 138164622.829 & 138165121.675 \\
\hline 1708.0 & 510.9 & -4062 & 4082 & 72 & 12 & 299.134 & 138163830.898 & 138164330.029 \\
\hline 1723.0 & 381.8 & -4338 & 5074 & 66 & 11 & 299.144 & 135134840.309 & 135135339.449 \\
\hline 1782.0 & 391.7 & 501 & 2757 & 68 & 11 & 299.142 & $13517 \quad 728.499$ & 135171227.639 \\
\hline 1813.0 & 498.7 & -236 & 2865 & 68 & 12 & 295.419 & 135171233.107 & 135171728.524 \\
\hline 1817.0 & 390.7 & -3462 & 2912 & 68 & 21 & 359.543 & 135171916.509 & 135172516.050 \\
\hline 1910.0 & 369.9 & -4432 & 4182 & 68 & 22 & 358.295 & 135172522.344 & 135173120.639 \\
\hline 1963.0 & 470.1 & -4474 & 4754 & 71 & 31 & 298.721 & 138153110.531 & 13815369.250 \\
\hline 2034.0 & 345.0 & -4022 & 3254 & 71 & 41 & 299.130 & 138154240.824 & 138154739.951 \\
\hline 2073.0 & 438.3 & -4508 & 4229 & 72 & 22 & 299.146 & 138165122.508 & 138165621.652 \\
\hline 2096.0 & 417.9 & -4435 & 4308 & 71 & 32 & 299.142 & 138153610.629 & 13815419.770 \\
\hline 2132.0 & 519.0 & -4606 & 4248 & 71 & 21 & 299.176 & 138152015.474 & 138152514.650 \\
\hline 2336.0 & 301.3 & -4525 & 4317 & 71 & 42 & 287.025 & 138154746.530 & 138155233.554 \\
\hline
\end{tabular}


308: Generator Power (kW)

\begin{tabular}{|c|c|c|c|c|c|c|c|c|}
\hline Mean & Std & $\operatorname{Min}$ & Max & Tape & Event & Time Length & Start Time & End Time \\
\hline-3.278 & 1.724 & -5.728 & 3.813 & 67 & 22 & 298.959 & 13516430.791 & 13516929.750 \\
\hline-2.667 & 1.716 & -5.635 & 3.340 & 75 & 22 & 289.576 & 20610335.072 & 206103754.646 \\
\hline-2.585 & 1.569 & -5.713 & 1.759 & 73 & 32 & 273.569 & 15312226.020 & 15312659.586 \\
\hline-2.312 & 2.354 & -5.830 & 6.754 & 73 & 42 & 299.532 & 153121250.225 & 153121749.755 \\
\hline-1.602 & 1.588 & -4.184 & 2.215 & 65 & 22 & 299.370 & 11594740.501 & 11595239.869 \\
\hline-1.561 & 1.904 & -20.790 & 3.574 & 73 & 41 & 298.510 & 15312750.870 & 153121249.378 \\
\hline-1.138 & 1.872 & -5.409 & 3.078 & 70 & 31 & 299.305 & 135214520.167 & 135215019.470 \\
\hline-0.907 & 1.382 & -3.575 & 4.482 & 70 & 41 & 299.230 & $1352157 \quad .617$ & 13522159.845 \\
\hline-0.617 & 2.571 & -4.551 & 7.077 & 70 & 32 & 299.151 & 135215020.307 & 135215519.458 \\
\hline-0.436 & 2.293 & -4.971 & 18.690 & 75 & 12 & 299.929 & 206101410.124 & 206101910.051 \\
\hline-0.324 & 3.788 & -6.276 & 7.673 & 69 & 32 & 293.222 & 135203546.647 & 135204039.869 \\
\hline-0.115 & 2.553 & -4.698 & 8.841 & 73 & 31 & 298.996 & 153115720.314 & 15312219.306 \\
\hline 0.047 & 2.182 & -22.060 & 6.383 & 75 & 21 & 299.812 & 2061028.205 & 2061033.013 \\
\hline 0.326 & 2.771 & -5.945 & 7.322 & 69 & 21 & 254.521 & 135201945.149 & 135202359.668 \\
\hline 0.610 & 2.367 & -4.638 & 7.478 & 69 & 31 & 299.679 & 135203040.268 & 135203539.945 \\
\hline 0.761 & 1.822 & -4.531 & 6.824 & 70 & 42 & 293.820 & 1352225.718 & 13522659.537 \\
\hline 1.071 & 2.083 & -3.523 & 5.842 & 67 & 21 & 299.545 & 135155930.414 & 13516429.957 \\
\hline 1.146 & 1.448 & -2.814 & 20.130 & 70 & 21 & 298.798 & 135213440.919 & 135213939.715 \\
\hline 1.324 & 2.569 & -3.731 & 9.262 & 70 & 22 & 254.371 & 135213945.583 & 135214359.952 \\
\hline 1.413 & 1.809 & -2.970 & 6.355 & 70 & 11 & 299.601 & 135212335.137 & 135212834.737 \\
\hline 1.626 & 2.872 & -3.781 & 20.780 & 73 & 22 & 292.729 & 153115146.543 & 153115639.272 \\
\hline 1.691 & 1.950 & -3.567 & 19.570 & 75 & 11 & 299.439 & 20610910.688 & 206101410.124 \\
\hline 1.749 & 2.613 & -3.287 & 9.297 & 65 & 11 & 299.439 & 11593115.541 & 11593614.980 \\
\hline 2.069 & 3.514 & -4.579 & 9.136 & 69 & 22 & 224.076 & 135202445.731 & 135202829.803 \\
\hline 2.154 & 2.002 & -2.155 & 7.931 & 65 & 21 & 299.355 & 11594240.316 & 11594739.669 \\
\hline 2.569 & 2.266 & -3.024 & 9.072 & 66 & 21 & 299.234 & 13514240.759 & 13514739.991 \\
\hline 2.688 & 2.701 & -3.911 & 9.668 & 65 & 12 & 299.224 & 11593615.822 & 11594115.046 \\
\hline 2.738 & 2.301 & -3.868 & 8.443 & 70 & 12 & 299.255 & 135212835.569 & 135213334.824 \\
\hline 2.982 & 3.020 & -23.690 & 11.230 & 72 & 42 & 289.056 & 138171430.447 & 138171919.501 \\
\hline 3.315 & 2.504 & -3.590 & 11.300 & 66 & 22 & 299.046 & 13514740.831 & 135141239.875 \\
\hline 4.024 & 2.711 & -1.600 & 11.530 & 66 & 32 & 298.883 & 135141910.642 & 13514249.523 \\
\hline 4.027 & 2.253 & -1.377 & 11.790 & 67 & 12 & 298.846 & 135155240.559 & 135155739.403 \\
\hline 4.765 & 2.334 & -1.162 & 18.430 & 67 & 11 & 299.499 & 135154740.226 & 135155239.723 \\
\hline 5.136 & 4.778 & -5.648 & 16.550 & 72 & 32 & 261.823 & 1381746.242 & 13817828.064 \\
\hline 6.109 & 2.874 & -3.367 & 13.780 & 67 & 31 & 299.288 & $1351611 \quad .492$ & 135161559.778 \\
\hline 6.214 & 3.169 & -1.385 & 14.070 & 66 & 31 & 299.449 & 135141410.599 & 135141910.046 \\
\hline 6.546 & 2.212 & -0.367 & 12.430 & 73 & 21 & 299.151 & 153114640.756 & 153115139.904 \\
\hline 6.946 & 3.189 & -1.440 & 14.050 & 73 & 12 & 300.008 & 153114050.094 & 153114550.101 \\
\hline 7.246 & 4.822 & -0.868 & 20.790 & 72 & 41 & 298.986 & 13817930.796 & 138171429.779 \\
\hline 7.385 & 5.030 & -4.790 & 17.470 & 72 & 11 & 299.750 & 138163330.441 & 138163830.191 \\
\hline 7.554 & 2.645 & -0.780 & 13.810 & 66 & 12 & 297.491 & 135135345.999 & 135135843.489 \\
\hline 7.979 & 2.479 & 1.003 & 14.500 & 67 & 32 & 299.149 & $1351616 \quad .526$ & 135162059.672 \\
\hline 9.578 & 3.063 & -0.543 & 16.270 & 73 & 11 & 298.742 & 153113550.765 & 153114049.504 \\
\hline 9.580 & 3.019 & -10.150 & 16.440 & 71 & 11 & 299.566 & 13815910.495 & 138151410.059 \\
\hline 9.839 & 3.394 & -20.920 & 18.630 & 71 & 12 & 299.574 & 138151410.641 & $13815 \quad 19 \quad 10.211$ \\
\hline 9.946 & 3.350 & 0.185 & 17.960 & 72 & 31 & 299.134 & $1381659 \quad .620$ & 13817359.754 \\
\hline 10.370 & 4.005 & -0.283 & 18.170 & 72 & 21 & 298.848 & 138164622.829 & 138165121.675 \\
\hline 10.740 & 2.550 & 2.868 & 17.050 & 66 & 11 & 299.144 & 135134840.309 & 135135339.449 \\
\hline 10.760 & 3.379 & 1.005 & 19.190 & 72 & 12 & 299.134 & 138163830.898 & 138164330.029 \\
\hline 11.230 & 2.587 & 2.583 & 16.900 & 68 & 11 & 299.142 & 13517728.499 & 135171227.639 \\
\hline 11.450 & 3.325 & -5.436 & 17.900 & 68 & 12 & 295.419 & 135171233.107 & 135171728.524 \\
\hline 11.510 & 2.553 & 1.608 & 16.980 & 68 & 21 & 359.543 & 135171916.509 & 135172516.050 \\
\hline 12.150 & 2.329 & 3.617 & 18.780 & 68 & 22 & 358.295 & 135172522.344 & 135173120.639 \\
\hline 12.420 & 3.074 & -26.520 & 20.350 & 71 & 31 & 298.721 & $1381531 \quad 10.531$ & 13815369.250 \\
\hline 12.970 & 2.201 & 3.802 & 19.900 & 71 & 41 & 299.130 & 138154240.824 & 138154739.951 \\
\hline 13.250 & 2.834 & 1.005 & 18.990 & 72 & 22 & 299.146 & 138165122.508 & 138165621.652 \\
\hline 13.330 & 2.679 & 2.105 & 20.850 & 71 & 32 & 299.142 & 138153610.629 & 13815419.770 \\
\hline 13.380 & 3.420 & 0.017 & 21.010 & 71 & 21 & 299.176 & 138152015.474 & 138152514.650 \\
\hline 14.950 & 1.825 & 5.734 & 20.500 & 71 & 42 & 287.025 & 138154746.530 & 138155233.554 \\
\hline
\end{tabular}


714: Normal Force Coefficient, $\mathrm{Cn}$, at $80 \%$ Span

\begin{tabular}{|c|c|c|c|c|c|c|c|c|}
\hline Mean & Std & Min & Max & Tape & Event & Time Length & Start Time & End Time \\
\hline-0.271 & 0.218 & -0.873 & 0.521 & 67 & 22 & 298.959 & 13516430.791 & 13516929.750 \\
\hline-0.183 & 0.186 & -5.730 & 13.800 & 75 & 22 & 289.576 & 20610335.072 & 206103754.646 \\
\hline-0.020 & 0.181 & -4.496 & 3.949 & 70 & 31 & 299.305 & 135214520.167 & 135215019.470 \\
\hline-0.007 & 0.067 & -7.506 & 12.540 & 73 & 32 & 273.569 & 15312226.020 & 15312659.586 \\
\hline-0.006 & 0.035 & -5.611 & 1.676 & 73 & 31 & 298.996 & 153115720.314 & 15312219.306 \\
\hline-0.001 & 0.035 & -3.394 & 3.843 & 73 & 22 & 292.729 & 153115146.543 & 153115639.272 \\
\hline-0.001 & 0.033 & -3.407 & 6.484 & 73 & 42 & 299.532 & 153121250.225 & 153121749.755 \\
\hline 0.001 & 0.032 & -3.731 & 4.135 & 73 & 21 & 299.151 & 153114640.756 & 153115139.904 \\
\hline 0.002 & 0.034 & -4.890 & 3.858 & 73 & 41 & 298.510 & 15312750.870 & 153121249.378 \\
\hline 0.003 & 0.037 & -9.800 & 2.623 & 73 & 12 & 300.008 & 153114050.094 & 153114550.101 \\
\hline 0.005 & 0.132 & -3.768 & 0.990 & 70 & 41 & 299.230 & $1352157 \quad .617$ & 13522159.845 \\
\hline 0.006 & 0.032 & -6.057 & 2.274 & 73 & 11 & 298.742 & 153113550.765 & 153114049.504 \\
\hline 0.020 & 0.227 & -5.227 & 0.880 & 70 & 32 & 299.151 & 135215020.307 & 135215519.458 \\
\hline 0.028 & 0.401 & -8.290 & 11.720 & 69 & 32 & 293.222 & 135203546.647 & 135204039.869 \\
\hline 0.031 & 0.203 & -8.457 & 5.109 & 65 & 22 & 299.370 & 11594740.501 & 11595239.869 \\
\hline 0.087 & 0.223 & -8.892 & 5.578 & 75 & 21 & 299.812 & $2061028 \quad .205$ & 2061033.013 \\
\hline 0.105 & 0.224 & -10.530 & 5.391 & 75 & 12 & 299.929 & 206101410.124 & 206101910.051 \\
\hline 0.127 & 0.301 & -9.096 & 10.870 & 69 & 21 & 254.521 & 135201945.149 & 135202359.668 \\
\hline 0.140 & 0.168 & -3.703 & 3.732 & 70 & 42 & 293.820 & 1352225.718 & 13522659.537 \\
\hline 0.146 & 0.247 & -8.958 & 9.929 & 69 & 31 & 299.679 & 135203040.268 & 135203539.945 \\
\hline 0.178 & 0.137 & -0.367 & 4.132 & 70 & 21 & 298.798 & 135213440.919 & 135213939.715 \\
\hline 0.180 & 0.245 & -10.280 & 11.930 & 70 & 22 & 254.371 & 135213945.583 & 135214359.952 \\
\hline 0.196 & 0.195 & -3.629 & 3.153 & 67 & 21 & 299.545 & 135155930.414 & 13516429.957 \\
\hline 0.199 & 0.162 & -3.746 & 6.149 & 70 & 11 & 299.601 & 135212335.137 & 135212834.737 \\
\hline 0.255 & 0.339 & -11.700 & 9.125 & 69 & 22 & 224.076 & 135202445.731 & 135202829.803 \\
\hline 0.290 & 0.194 & -3.630 & 4.238 & 70 & 12 & 299.255 & 135212835.569 & 135213334.824 \\
\hline 0.308 & 0.206 & -23.270 & 14.220 & 75 & 11 & 299.439 & 20610910.688 & 206101410.124 \\
\hline 0.324 & 0.217 & -9.943 & 12.120 & 66 & 21 & 299.234 & 13514240.759 & 13514739.991 \\
\hline 0.343 & 0.295 & -11.110 & 13.050 & 72 & 42 & 289.056 & 138171430.447 & 138171919.501 \\
\hline 0.370 & 0.226 & -7.351 & 7.474 & 66 & 22 & 299.046 & 13514740.831 & 135141239.875 \\
\hline 0.412 & 0.236 & -3.636 & 4.294 & 65 & 11 & 299.439 & 11593115.541 & 11593614.980 \\
\hline 0.420 & 0.240 & -13.130 & 7.611 & 66 & 32 & 298.883 & 135141910.642 & 13514249.523 \\
\hline 0.423 & 0.193 & -3.704 & 4.881 & 65 & 21 & 299.355 & 11594240.316 & 11594739.669 \\
\hline 0.435 & 0.191 & -4.390 & 4.103 & 67 & 12 & 298.846 & 135155240.559 & 135155739.403 \\
\hline 0.463 & 0.302 & -9.957 & 11.410 & 65 & 12 & 299.224 & 11593615.822 & 11594115.046 \\
\hline 0.478 & 0.387 & -8.492 & 7.271 & 72 & 32 & 261.823 & 1381746.242 & 13817828.064 \\
\hline 0.494 & 0.195 & -3.422 & 10.950 & 67 & 11 & 299.499 & $135 \cdot 154740.226$ & 135155239.723 \\
\hline 0.539 & 0.183 & -8.926 & 10.910 & 66 & 12 & 297.491 & 135135345.999 & 135135843.489 \\
\hline 0.562 & 0.242 & -9.131 & 9.879 & 66 & 31 & 299.449 & 135141410.599 & 135141910.046 \\
\hline 0.571 & 0.232 & -4.434 & 3.714 & 67 & 31 & 299.288 & 1351611.492 & 135161559.778 \\
\hline 0.615 & 0.350 & -10.510 & 10.690 & 72 & 41 & 298.986 & 13817930.796 & 138171429.779 \\
\hline 0.631 & 0.168 & -6.226 & 6.268 & 66 & 11 & 299.144 & 135134840.309 & 135135339.449 \\
\hline 0.653 & 0.388 & -5.730 & 4.334 & 72 & 11 & 299.750 & 138163330.441 & 138163830.191 \\
\hline 0.691 & 0.182 & -3.299 & 4.310 & 67 & 32 & 299.149 & $1351616 \quad .526$ & 135162059.672 \\
\hline 0.779 & 0.247 & -10.270 & 11.370 & 71 & 11 & 299.566 & 13815910.495 & 138151410.059 \\
\hline 0.782 & 0.245 & -10.850 & 8.860 & 71 & 12 & 299.574 & 138151410.641 & 138151910.211 \\
\hline 0.797 & 0.254 & -7.624 & 8.327 & 72 & 31 & 299.134 & 1381659.620 & 13817359.754 \\
\hline 0.809 & 0.280 & -7.443 & 7.380 & 72 & 21 & 298.848 & 138164622.829 & 138165121.675 \\
\hline 0.831 & 0.226 & -3.235 & 1.557 & 68 & 12 & 295.419 & 135171233.107 & 135171728.524 \\
\hline 0.839 & 0.172 & -3.502 & 3.439 & 68 & 11 & 299.142 & 13517728.499 & 135171227.639 \\
\hline 0.852 & 0.188 & -1.641 & 3.107 & 68 & 21 & 359.543 & 135171916.509 & 135172516.050 \\
\hline 0.852 & 0.244 & -9.865 & 9.573 & 72 & 12 & 299.134 & 138163830.898 & 138164330.029 \\
\hline 0.871 & 0.221 & -10.470 & 11.170 & 68 & 22 & 358.295 & 135172522.344 & 135173120.639 \\
\hline 0.881 & 0.235 & -7.405 & 8.669 & 71 & 31 & 298.721 & 138153110.531 & 13815369.250 \\
\hline 0.894 & 0.188 & -7.917 & 5.899 & 71 & 41 & 299.130 & 138154240.824 & 138154739.951 \\
\hline 0.914 & 0.207 & -7.342 & 6.957 & 71 & 32 & 299.142 & 138153610.629 & 13815419.770 \\
\hline 0.921 & 0.256 & -9.768 & 10.010 & 71 & 21 & 299.176 & 138152015.474 & 138152514.650 \\
\hline 0.952 & 0.249 & -5.917 & 6.340 & 72 & 22 & 299.146 & 138165122.508 & 138165621.652 \\
\hline 0.972 & 0.206 & -10.710 & 4.258 & 71 & 42 & 287.025 & 138154746.530 & 138155233.554 \\
\hline
\end{tabular}


715: Normal Force Coefficient, $\mathrm{Cn}$, at $63.3 \%$ Span

\begin{tabular}{|c|c|c|c|c|c|c|c|c|}
\hline Mean & Std & Min & $\operatorname{Max}$ & Tape & Event & Time Length & Start Time & End Time \\
\hline-0.125 & 0.275 & -0.776 & 0.825 & 67 & 22 & 298.959 & 13516430.791 & 13516929.750 \\
\hline 0.035 & 0.234 & -7.246 & 8.634 & 75 & 22 & 289.576 & 20610335.072 & 206103754.646 \\
\hline 0.039 & 0.336 & -0.914 & 1.082 & 73 & 42 & 299.532 & 153121250.225 & 153121749.755 \\
\hline 0.046 & 0.245 & -0.904 & 4.449 & 73 & 32 & 273.569 & 15312226.020 & 15312659.586 \\
\hline 0.173 & 0.268 & -0.688 & 1.033 & 73 & 41 & 298.510 & 15312750.870 & 153121249.378 \\
\hline 0.180 & 0.250 & -0.392 & 5.154 & 65 & 22 & 299.370 & 11594740.501 & 11595239.869 \\
\hline 0.187 & 0.234 & -2.491 & 13.490 & 70 & 31 & 299.305 & 135214520.167 & 135215019.470 \\
\hline 0.219 & 0.169 & -0.471 & 0.939 & 70 & 41 & 299.230 & $1352157 \quad .617$ & 13522159.845 \\
\hline 0.226 & 0.274 & -0.531 & 1.059 & 70 & 32 & 299.151 & 135215020.307 & 135215519.458 \\
\hline 0.228 & 0.447 & -2.056 & 5.301 & 69 & 32 & 293.222 & 135203546.647 & 135204039.869 \\
\hline 0.351 & 0.277 & -0.474 & 1.300 & 73 & 31 & 298.996 & 153115720.314 & 15312219.306 \\
\hline 0.362 & 0.300 & -0.861 & 1.111 & 69 & 21 & 254.521 & 135201945.149 & 135202359.668 \\
\hline 0.366 & 0.266 & -1.661 & 12.130 & 75 & 21 & 299.812 & 2061028.205 & 2061033.013 \\
\hline 0.371 & 0.267 & -6.256 & 6.795 & 75 & 12 & 299.929 & 206101410.124 & 206101910.051 \\
\hline 0.387 & 0.263 & -5.753 & 2.577 & 69 & 31 & 299.679 & 135203040.268 & 135203539.945 \\
\hline 0.387 & 0.201 & -9.448 & 1.052 & 70 & 42 & 293.820 & 1352225.718 & 13522659.537 \\
\hline 0.410 & 0.161 & -0.259 & 4.271 & 66 & 12 & 297.491 & 135135345.999 & 135135843.489 \\
\hline 0.423 & 0.241 & -0.362 & 1.230 & 70 & 22 & 254.371 & 135213945.583 & 135214359.952 \\
\hline 0.431 & 0.160 & -2.998 & 1.008 & 70 & 21 & 298.798 & 135213440.919 & 135213939.715 \\
\hline 0.454 & 0.235 & -0.389 & 1.780 & 67 & 21 & 299.545 & 135155930.414 & 13516429.957 \\
\hline 0.463 & 0.182 & -0.220 & 0.976 & 70 & 11 & 299.601 & 135212335.137 & 135212834.737 \\
\hline 0.507 & 0.334 & -2.966 & 1.270 & 69 & 22 & 224.076 & 135202445.731 & 135202829.803 \\
\hline 0.536 & 0.268 & -2.924 & 1.326 & 73 & 22 & 292.729 & 153115146.543 & 153115639.272 \\
\hline 0.558 & 0.217 & -0.436 & 3.295 & 70 & 12 & 299.255 & 135212835.569 & 135213334.824 \\
\hline 0.584 & 0.215 & -0.729 & 3.512 & 66 & 21 & 299.234 & 13514240.759 & 13514739.991 \\
\hline 0.597 & 0.211 & -0.258 & 1.435 & 66 & 11 & 299.144 & 135134840.309 & 135135339.449 \\
\hline 0.598 & 0.291 & -11.840 & 1.306 & 72 & 42 & 289.056 & 138171430.447 & 138171919.501 \\
\hline 0.605 & 0.202 & -6.318 & 7.910 & 75 & 11 & 299.439 & 20610910.688 & 206101410.124 \\
\hline 0.619 & 0.210 & -0.294 & 1.349 & 66 & 22 & 299.046 & 13514740.831 & 135141239.875 \\
\hline 0.633 & 0.254 & -3.763 & 1.500 & 65 & 11 & 299.439 & 11593115.541 & 11593614.980 \\
\hline 0.642 & 0.201 & -2.252 & 1.300 & 65 & 21 & 299.355 & 11594240.316 & 11594739.669 \\
\hline 0.663 & 0.191 & -0.052 & 1.271 & 66 & 32 & 298.883 & 135141910.642 & 13514249.523 \\
\hline 0.694 & 0.252 & -8.857 & 3.573 & 65 & 12 & 299.224 & 11593615.822 & 11594115.046 \\
\hline 0.696 & 0.388 & -1.183 & 2.446 & 72 & 32 & 261.823 & 1381746.242 & 13817828.064 \\
\hline 0.702 & 0.194 & -3.070 & 1.402 & 67 & 12 & 298.846 & 135155240.559 & 135155739.403 \\
\hline 0.758 & 0.191 & -7.833 & 1.845 & 67 & 11 & 299.499 & 135154740.226 & 135155239.723 \\
\hline 0.771 & 0.191 & -0.745 & 2.051 & 66 & 31 & 299.449 & 135141410.599 & 135141910.046 \\
\hline 0.810 & 0.236 & -0.624 & 1.662 & 67 & 31 & 299.288 & 1351611.492 & 135161559.778 \\
\hline 0.841 & 0.399 & -13.380 & 2.290 & 72 & 11 & 299.750 & 138163330.441 & 138163830.191 \\
\hline 0.850 & 0.310 & -2.834 & 2.209 & 72 & 41 & 298.986 & 13817930.796 & 138171429.779 \\
\hline 0.887 & 0.215 & -0.014 & 3.066 & 73 & 12 & 300.008 & 153114050.094 & 153114550.101 \\
\hline 0.889 & 0.167 & -5.407 & 2.309 & 73 & 21 & 299.151 & 153114640.756 & 153115139.904 \\
\hline 0.904 & 0.175 & 0.111 & 2.689 & 67 & 32 & 299.149 & 1351616.526 & 135162059.672 \\
\hline 0.978 & 0.217 & -4.855 & 6.848 & 71 & 11 & 299.566 & 13815910.495 & 138151410.059 \\
\hline 0.982 & 0.228 & -0.955 & 2.210 & 71 & 12 & 299.574 & 138151410.641 & 138151910.211 \\
\hline 0.991 & 0.261 & -0.029 & 2.110 & 72 & 31 & 299.134 & $1381659 \quad .620$ & 13817359.754 \\
\hline 1.003 & 0.197 & -2.586 & 2.296 & 73 & 11 & 298.742 & 153113550.765 & 153114049.504 \\
\hline 1.015 & 0.252 & -0.324 & 2.040 & 68 & 12 & 295.419 & 135171233.107 & 135171728.524 \\
\hline 1.019 & 0.280 & -6.479 & 2.309 & 72 & 21 & 298.848 & 138164622.829 & 138165121.675 \\
\hline 1.020 & 0.200 & -7.818 & 6.760 & 68 & 11 & 299.142 & 13517728.499 & 135171227.639 \\
\hline 1.030 & 0.218 & -3.810 & 2.289 & 68 & 21 & 359.543 & 135171916.509 & 135172516.050 \\
\hline 1.030 & 0.238 & -2.785 & 2.621 & 72 & 12 & 299.134 & 138163830.898 & 138164330.029 \\
\hline 1.048 & 0.209 & -6.577 & 1.950 & 68 & 22 & 358.295 & 135172522.344 & 135173120.639 \\
\hline 1.069 & 0.245 & -5.900 & 3.685 & 71 & 31 & 298.721 & $1381531 \quad 10.531$ & 13815369.250 \\
\hline 1.090 & 0.223 & -4.164 & 8.273 & 71 & 41 & 299.130 & 138154240.824 & 138154739.951 \\
\hline 1.095 & 0.232 & -3.391 & 5.846 & 71 & 32 & 299.142 & 138153610.629 & 13815419.770 \\
\hline 1.096 & 0.263 & -2.584 & 7.424 & 71 & 21 & 299.176 & 138152015.474 & 138152514.650 \\
\hline 1.141 & 0.270 & -0.121 & 2.434 & 72 & 22 & 299.146 & 138165122.508 & 138165621.652 \\
\hline 1.158 & 0.232 & -1.732 & 5.435 & 71 & 42 & 287.025 & 138154746.530 & 138155233.554 \\
\hline
\end{tabular}


716: Normal Force Coefficient, $\mathrm{Cn}$, at $\mathbf{4 6 . 6 \%}$ Span

\begin{tabular}{|c|c|c|c|c|c|c|c|c|}
\hline Mean & Std & Min & $\operatorname{Max}$ & Tape & Event & Time Length & Start Time & End Time \\
\hline 0.082 & 0.352 & -5.255 & 1.303 & 67 & 22 & 298.959 & $13516 \quad 430.791$ & 13516929.750 \\
\hline 0.248 & 0.187 & -4.030 & 4.637 & 66 & 12 & 297.491 & 135135345.999 & 135135843.489 \\
\hline 0.272 & 0.398 & -0.935 & 2.843 & 73 & 42 & 299.532 & 153121250.225 & 153121749.755 \\
\hline 0.311 & 0.304 & -1.654 & 7.114 & 73 & 32 & 273.569 & 15312226.020 & 15312659.586 \\
\hline 0.327 & 0.285 & -8.915 & 10.830 & 75 & 22 & 289.576 & 20610335.072 & 206103754.646 \\
\hline 0.339 & 0.183 & -3.560 & 4.077 & 66 & 11 & 299.144 & 135134840.309 & 135135339.449 \\
\hline 0.410 & 0.324 & -6.519 & 5.699 & 65 & 22 & 299.370 & 11594740.501 & 11595239.869 \\
\hline 0.440 & 0.312 & -4.136 & 1.455 & $\overline{73}$ & 41 & 298.510 & 15312750.870 & 153121249.378 \\
\hline 0.468 & 0.480 & -1.725 & 4.902 & 69 & 32 & 293.222 & 135203546.647 & 135204039.869 \\
\hline 0.500 & 0.293 & -0.714 & 4.268 & 70 & 31 & 299.305 & 135214520.167 & 135215019.470 \\
\hline 0.530 & 0.308 & -3.396 & 1.431 & 70 & 32 & 299.151 & 135215020.307 & 135215519.458 \\
\hline 0.550 & 0.209 & -2.758 & 1.306 & 70 & 41 & 299.230 & $1352157 \quad .617$ & 13522159.845 \\
\hline 0.632 & 0.312 & -8.605 & 7.854 & 69 & 21 & 254.521 & 135201945.149 & 135202359.668 \\
\hline 0.636 & 0.283 & -11.270 & 7.392 & 73 & 31 & 298.996 & 153115720.314 & 15312219.306 \\
\hline 0.652 & 0.270 & -10.900 & 8.417 & 75 & 12 & 299.929 & 206101410.124 & 206101910.051 \\
\hline 0.668 & 0.262 & -7.538 & 9.289 & 75 & 21 & 299.812 & 2061028.205 & $2061033 \quad .013$ \\
\hline 0.697 & 0.280 & -5.433 & 8.812 & 69 & 31 & 299.679 & 135203040.268 & 135203539.945 \\
\hline 0.714 & 0.218 & -0.593 & 1.490 & 70 & 42 & 293.820 & 1352225.718 & 13522659.537 \\
\hline 0.737 & 0.242 & -3.498 & 9.847 & 70 & 22 & 254.371 & 135213945.583 & 135214359.952 \\
\hline 0.742 & 0.227 & -0.673 & 1.442 & 67 & 21 & 299.545 & 135155930.414 & 13516429.957 \\
\hline 0.752 & 0.324 & -7.579 & 6.002 & 69 & 22 & 224.076 & 135202445.731 & 135202829.803 \\
\hline 0.760 & 0.175 & -5.552 & 1.735 & 70 & 21 & 298.798 & 135213440.919 & 135213939.715 \\
\hline 0.781 & 0.177 & -0.112 & 1.334 & 70 & 11 & 299.601 & 135212335.137 & 135212834.737 \\
\hline 0.785 & 0.239 & -0.116 & 1.665 & 73 & 22 & 292.729 & 153115146.543 & 153115639.272 \\
\hline 0.826 & 0.285 & -8.504 & 8.243 & 72 & 42 & 289.056 & 138171430.447 & 138171919.501 \\
\hline 0.831 & .0 .228 & -6.762 & 10.640 & 66 & 21 & 299.234 & 13514240.759 & 13514739.991 \\
\hline 0.838 & 0.237 & -5.574 & 7.617 & 65 & 11 & 299.439 & 11593115.541 & 11593614.980 \\
\hline 0.845 & 0.219 & -0.387 & 2.011 & 70 & 12 & 299.255 & 135212835.569 & 135213334.824 \\
\hline 0.851 & 0.188 & -7.403 & 6.608 & 75 & 11 & 299.439 & 20610910.688 & 206101410.124 \\
\hline 0.870 & 0.195 & -4.139 & 8.993 & 65 & 21 & 299.355 & 11594240.316 & 11594739.669 \\
\hline 0.877 & 0.217 & -7.468 & 5.002 & 66 & 22 & 299.046 & 13514740.831 & 135141239.875 \\
\hline 0.879 & 0.355 & -1.250 & 2.482 & 72 & 32 & 261.823 & 1381746.242 & 13817828.064 \\
\hline 0.884 & 0.239 & -7.646 & 8.582 & 65 & 12 & 299.224 & 11593615.822 & 11594115.046 \\
\hline 0.896 & 0.183 & -0.842 & 1.778 & 66 & 32 & 298.883 & 135141910.642 & 13514249.523 \\
\hline 0.927 & 0.197 & -0.146 & 7.435 & 67 & 12 & 298.846 & 135155240.559 & 135155739.403 \\
\hline 0.944 & 0.199 & -6.073 & 7.698 & 66 & 31 & 299.449 & 135141410.599 & 135141910.046 \\
\hline 0.949 & 0.346 & -6.524 & 8.133 & 72 & 11 & 299.750 & 138163330.441 & 138163830.191 \\
\hline $0 . \overline{962}$ & 0.202 & -2.726 & 2.019 & 67 & 11 & 299.499 & 135154740.226 & 135155239.723 \\
\hline 0.962 & 0.256 & -1.835 & 2.109 & 67 & 31 & 299.288 & 1351611.492 & 135161559.778 \\
\hline 0.976 & 0.245 & -6.870 & 9.923 & 72 & 41 & 298.986 & 13817930.796 & 138171429.779 \\
\hline 0.984 & 0.229 & -5.819 & 8.892 & 73 & 12 & 300.008 & 153114050.094 & 153114550.101 \\
\hline 0.986 & 0.217 & -6.967 & 7.700 & 73 & 21 & 299.151 & 153114640.756 & 153115139.904 \\
\hline 0.998 & 0.219 & -1.009 & 5.214 & 67 & 32 & 299.149 & 1351616.526 & 135162059.672 \\
\hline 1.007 & 0.201 & -6.097 & 6.096 & 73 & 11 & 298.742 & 153113550.765 & 153114049.504 \\
\hline 1.027 & 0.235 & -7.025 & 6.884 & 71 & 11 & 299.566 & 13815910.495 & 138151410.059 \\
\hline 1.028 & 0.191 & -5.981 & 5.734 & 68 & 11 & 299.142 & 13517728.499 & 135171227.639 \\
\hline 1.037 & 0.238 & -6.504 & 9.532 & 71 & 12 & 299.574 & 138151410.641 & 138151910.211 \\
\hline 1.053 & 0.237 & -0.722 & 2.560 & 68 & 12 & 295.419 & $13517 \quad 1233.107$ & 135171728.524 \\
\hline 1.057 & 0.258 & -5.587 & 3.830 & 72 & 31 & 299.134 & $1381659 \quad .620$ & 13817359.754 \\
\hline 1.060 & 0.246 & -7.820 & 7.253 & 72 & 21 & 298.848 & 138164622.829 & 138165121.675 \\
\hline 1.061 & 0.222 & 0.146 & 2.434 & 72 & 12 & 299.134 & 138163830.898 & 138164330.029 \\
\hline 1.069 & 0.211 & -6.404 & 7.719 & 68 & 22 & 358.295 & 135172522.344 & 135173120.639 \\
\hline 1.075 & 0.225 & -2.281 & 3.701 & 68 & 21 & 359.543 & 135171916.509 & 135172516.050 \\
\hline 1.080 & 0.226 & -5.274 & 7.053 & 71 & 31 & 298.721 & 138153110.531 & 13815369.250 \\
\hline 1.080 & 0.221 & -7.243 & 7.521 & 71 & 41 & 299.130 & 138154240.824 & 138154739.951 \\
\hline 1.101 & 0.224 & -6.084 & 7.069 & 71 & 32 & 299.142 & 138153610.629 & 13815419.770 \\
\hline 1.115 & 0.237 & -4.302 & 6.724 & 71 & 21 & 299.176 & 138152015.474 & 138152514.650 \\
\hline 1.149 & 0.250 & -4.109 & 7.727 & 72 & 22 & 299.146 & 138165122.508 & 138165621.652 \\
\hline 1.153 & 0.208 & -5.289 & 7.021 & 71 & 42 & 287.025 & 138154746.530 & 138155233.554 \\
\hline
\end{tabular}


717: Normal Force Coefficient, Cn, at 30\% Span

\begin{tabular}{|c|c|c|c|c|c|c|c|c|}
\hline Mean & Std & Min & Max & Tape & Event & Time Length & Start Time & End Time \\
\hline 0.359 & 0.474 & -1.983 & 1.978 & 67 & 22 & 298.959 & 13516430.791 & 13516929.750 \\
\hline 0.456 & 0.446 & -10.080 & 13.440 & 66 & 12 & 297.491 & 135135345.999 & 135135843.489 \\
\hline 0.638 & 0.543 & -1.032 & 3.998 & 73 & 42 & 299.532 & 153121250.225 & 153121749.755 \\
\hline 0.658 & 0.415 & -8.106 & 5.531 & 66 & 11 & 299.144 & 135134840.309 & 135135339.449 \\
\hline 0.664 & 0.413 & -3.884 & 31.370 & 73 & 32 & 273.569 & 15312226.020 & 15312659.586 \\
\hline 0.677 & 0.449 & -11.370 & 18.930 & 65 & 22 & 299.370 & 11594740.501 & 11595239.869 \\
\hline 0.721 & 0.387 & -19.900 & 14.160 & 75 & 22 & 289.576 & 20610335.072 & 206103754.646 \\
\hline 0.849 & 0.677 & -10.930 & 6.875 & 69 & 32 & 293.222 & 135203546.647 & 135204039.869 \\
\hline 0.851 & 0.425 & -3.816 & 2.616 & 73 & 41 & 298.510 & 15312750.870 & 153121249.378 \\
\hline 0.892 & 0.388 & -11.620 & 1.959 & 70 & 31 & 299.305 & 135214520.167 & 135215019.470 \\
\hline 0.947 & 0.424 & -6.557 & 2.531 & 70 & 32 & 299.151 & 135215020.307 & 135215519.458 \\
\hline 0.988 & 0.284 & -11.700 & 5.331 & 70 & 41 & 299.230 & $\begin{array}{lll}1352157 & .617\end{array}$ & 13522159.845 \\
\hline 1.080 & 0.441 & -11.550 & 8.224 & 69 & 21 & 254.521 & 135201945.149 & 135202359.668 \\
\hline 1.106 & 0.430 & -25.220 & 18.400 & $\overline{73}$ & 31 & 298.996 & 153115720.314 & 15312219.306 \\
\hline 1.112 & 0.420 & -13.140 & 21.400 & 75 & 12 & 299.929 & 206101410.124 & 206101910.051 \\
\hline 1.122 & 0.410 & -1.392 & 14.220 & 69 & 31 & 299.679 & 135203040.268 & 135203539.945 \\
\hline 1.125 & 0.375 & -14.020 & 17.990 & 75 & 21 & 299.812 & $2061028 \quad .205$ & 2061033.013 \\
\hline 1.194 & 0.333 & -0.447 & 2.603 & 70 & 42 & 293.820 & 1352225.718 & 13522659.537 \\
\hline 1.242 & 0.381 & -12.020 & 22.070 & 70 & 22 & 254.371 & 135213945.583 & 135214359.952 \\
\hline 1.243 & 0.295 & -0.937 & 6.137 & 70 & 21 & 298.798 & 135213440.919 & 135213939.715 \\
\hline 1.256 & 0.349 & -0.141 & 2.673 & 67 & 21 & 299.545 & 135155930.414 & 13516429.957 \\
\hline 1.259 & 0.491 & -16.530 & 8.314 & 69 & 22 & 224.076 & 135202445.731 & 135202829.803 \\
\hline 1.272 & 0.301 & 0.010 & 12.270 & 70 & 11 & 299.601 & 135212335.137 & 135212834.737 \\
\hline 1.300 & 0.399 & -0.076 & 2.883 & 73 & 22 & 292.729 & 153115146.543 & 153115639.272 \\
\hline 1.341 & 0.391 & -12.440 & 15.040 & 66 & 21 & 299.234 & 13514240.759 & 13514739.991 \\
\hline 1.371 & 0.466 & -10.040 & 13.690 & 65 & 11 & 299.439 & 11593115.541 & 1159.3614 .980 \\
\hline 1.380 & 0.495 & -19.180 & 11.280 & 72 & 42 & 289.056 & 138171430.447 & 138171919.501 \\
\hline 1.384 & 0.360 & -0.664 & 2.739 & 70 & 12 & 299.255 & 135212835.569 & 135213334.824 \\
\hline 1.389 & 0.390 & -8.703 & 14.190 & 65 & 21 & 299.355 & 11594240.316 & 11594739.669 \\
\hline 1.398 & 0.333 & -12.710 & 10.740 & 75 & 11 & 299.439 & 20610910.688 & 206101410.124 \\
\hline 1.456 & 0.384 & -16.880 & 13.290 & 66 & 22 & 299.046 & 13514740.831 & 135141239.875 \\
\hline 1.491 & 0.661 & -4.345 & 6.512 & 72 & 32 & 261.823 & 1381746.242 & 13817828.064 \\
\hline 1.514 & 0.366 & 0.233 & 6.853 & 66 & 32 & 298.883 & 135141910.642 & 13514249.523 \\
\hline 1.523 & 0.550 & -18.910 & 14.940 & 65 & 12 & 299.224 & 11593615.822 & 11594115.046 \\
\hline 1.571 & 0.368 & 0.110 & 3.499 & 67 & 12 & 298.846 & 135155240.559 & 135155739.403 \\
\hline 1.666 & 0.364 & -4.576 & 3.751 & 67 & 11 & 299.499 & 135154740.226 & 135155239.723 \\
\hline 1.719 & 0.433 & -7.246 & 13.160 & 66 & 31 & 299.449 & 135141410.599 & 135141910.046 \\
\hline 1.719 & 0.477 & -0.516 & 3.683 & 67 & 31 & 299.288 & 1351611.492 & 135161559.778 \\
\hline 1.727 & 0.576 & -9.730 & 11.370 & 72 & 41 & 298.986 & 13817930.796 & 138171429.779 \\
\hline 1.809 & 0.419 & -15.000 & 18.680 & 73 & 21 & 299.151 & 153114640.756 & 153115139.904 \\
\hline 1.829 & 0.696 & -13.410 & 28.350 & 72 & 11 & 299.750 & 138163330.441 & 138163830.191 \\
\hline 1.869 & 0.487 & -6.571 & 19.130 & 73 & 12 & 300.008 & 153114050.094 & 153114550.101 \\
\hline 1.875 & 0.550 & -7.534 & 15.520 & 72 & 31 & 299.134 & 1381659.620 & 13817359.754 \\
\hline 1.927 & 0.436 & 0.353 & 3.983 & 67 & 32 & 299.149 & $1351616 \quad .526$ & 135162059.672 \\
\hline 1.986 & 0.562 & -6.926 & 10.380 & 72 & 21 & 298.848 & 138164622.829 & 138165121.675 \\
\hline 2.005 & 0.519 & -8.948 & 16.020 & 71 & 12 & 299.574 & 138151410.641 & 138151910.211 \\
\hline 2.010 & 0.574 & -2.951 & 8.673 & 72 & 22 & 299.146 & 138165122.508 & 138165621.652 \\
\hline 2.031 & 0.560 & -14.400 & 22.030 & 71 & 21 & 299.176 & 138152015.474 & 138152514.650 \\
\hline 2.039 & 0.511 & -5.426 & 4.347 & 72 & 12 & 299.134 & $138163 \overline{3830.898}$ & 138164330.029 \\
\hline 2.039 & 0.537 & -7.816 & 13.000 & 71 & 42 & 287.025 & 138154746.530 & 138155233.554 \\
\hline 2.045 & 0.507 & -13.480 & 17.090 & 71 & 11 & 299.566 & 13815910.495 & 138151410.059 \\
\hline 2.046 & 0.514 & -4.068 & 10.530 & 71 & 32 & 299.142 & 138153610.629 & 13815419.770 \\
\hline 2.079 & 0.552 & -8.741 & 9.170 & 71 & 31 & 298.721 & 138153110.531 & 13815369.250 \\
\hline 2.082 & 0.458 & -17.110 & 11.670 & 73 & 11 & 298.742 & 153113550.765 & 153114049.504 \\
\hline 2.090 & 0.558 & -4.829 & 4.318 & 68 & 12 & 295.419 & $13517 \quad 1233.107$ & 135171728.524 \\
\hline 2.107 & 0.519 & -11.290 & 10.940 & 71 & 41 & 299.130 & $138154240: 824$ & 138154739.951 \\
\hline 2.136 & 0.503 & 0.308 & 5.807 & 68 & 21 & 359.543 & 135171916.509 & 135172516.050 \\
\hline 2.175 & 0.449 & -3.022 & 4.355 & 68 & 11 & 299.142 & 13517728.499 & 135171227.639 \\
\hline 2.181 & 0.485 & -10.840 & 13.470 & 68 & 22 & 358.295 & 135172522.344 & 135173120.639 \\
\hline
\end{tabular}


718: Tangent Force Coefficient, $\mathrm{Ct}$, at $80 \%$ Span

\begin{tabular}{|c|c|c|c|c|c|c|c|c|}
\hline Mean & Std & Min & $\operatorname{Max}$ & Tape & Event & Time Length & Start Time & End Time \\
\hline-0.006 & 0.005 & -0.205 & 0.538 & 70 & 41 & 299.230 & $1352157 \quad .617$ & 13522159.845 \\
\hline-0.005 & 0.013 & -1.528 & 1.637 & 65 & 22 & 299.370 & 11594740.501 & 11595239.869 \\
\hline-0.004 & 0.007 & -0.249 & 0.371 & 70 & 31 & 299.305 & 135214520.167 & 135215019.470 \\
\hline-0.004 & 0.010 & -0.667 & 0.500 & 75 & 21 & 299.812 & 2061028.205 & $2061033 \quad .013$ \\
\hline-0.003 & 0.008 & -0.094 & 0.670 & 70 & 42 & 293.820 & 1352225.718 & 13522659.537 \\
\hline-0.003 & 0.008 & -0.652 & 0.983 & 70 & 21 & 298.798 & 135213440.919 & 135213939.715 \\
\hline-0.002 & 0.022 & -1.728 & 1.535 & 69 & 31 & 299.679 & 135203040.268 & 135203539.945 \\
\hline-0.002 & 0.010 & -0.425 & 0.187 & 70 & 11 & 299.601 & 135212335.137 & 135212834.737 \\
\hline-0.002 & 0.011 & -0.971 & 0.501 & 70 & 32 & 299.151 & 135215020.307 & 135215519.458 \\
\hline-0.002 & 0.006 & -0.218 & 1.497 & 73 & 21 & 299.151 & 153114640.756 & 153115139.904 \\
\hline-0.002 & 0.015 & -1.011 & 3.286 & 75 & 22 & 289.576 & 20610335.072 & 206103754.646 \\
\hline-0.002 & 0.004 & -0.360 & 0.511 & 73 & 22 & 292.729 & 153115146.543 & 153115639.272 \\
\hline-0.001 & 0.011 & -0.394 & 0.438 & 67 & 21 & 299.545 & 135155930.414 & 13516429.957 \\
\hline 0.000 & 0.013 & -0.856 & 1.474 & 75 & 12 & 299.929 & 206101410.124 & 206101910.051 \\
\hline 0.000 & 0.005 & -0.286 & 0.566 & 73 & 31 & 298.996 & 153115720.314 & 15312219.306 \\
\hline 0.000 & 0.009 & -1.190 & 1.001 & 73 & 32 & 273.569 & 15312226.020 & 15312659.586 \\
\hline 0.000 & 0.004 & -0.402 & 0.535 & 73 & 11 & 298.742 & 153113550.765 & 153114049.504 \\
\hline 0.001 & 0.004 & -0.800 & 0.070 & 73 & 12 & 300.008 & 153114050.094 & 153114550.101 \\
\hline 0.002 & 0.023 & -1.591 & 1.215 & 70 & 22 & 254.371 & 135213945.583 & 135214359.952 \\
\hline 0.002 & 0.007 & -0.963 & 1.488 & 73 & 41 & 298.510 & 15312750.870 & 153121249.378 \\
\hline 0.002 & 0.029 & -1.839 & 1.274 & 69 & 21 & 254.521 & 135201945.149 & 135202359.668 \\
\hline 0.003 & 0.009 & -1.911 & 2.193 & 73 & 42 & 299.532 & 153121250.225 & 153121749.755 \\
\hline 0.007 & 0.017 & -1.258 & 0.474 & 70 & 12 & 299.255 & 135212835.569 & 135213334.824 \\
\hline 0.008 & 0.019 & -0.991 & 1.650 & 75 & 11 & 299.439 & 20610910.688 & 206101410.124 \\
\hline 0.008 & 0.030 & -1.812 & 1.740 & 69 & 32 & 293.222 & 135203546.647 & 135204039.869 \\
\hline 0.008 & 0.014 & -0.026 & 0.073 & 67 & 22 & 298.959 & 13516430.791 & 13516929.750 \\
\hline 0.009 & 0.023 & -1.915 & 1.550 & 66 & 21 & 299.234 & 13514240.759 & 13514739.991 \\
\hline 0.012 & 0.038 & -2.635 & 1.982 & 69 & 22 & 224.076 & 135202445.731 & 135202829.803 \\
\hline 0.015 & 0.028 & -1.482 & 1.292 & 66 & 22 & 299.046 & 13514740.831 & 135141239.875 \\
\hline 0.016 & 0.035 & -1.526 & 1.866 & 72 & 42 & 289.056 & 138171430.447 & 138171919.501 \\
\hline 0.018 & 0.026 & -0.873 & 0.902 & 65 & 21 & 299.355 & 11594240.316 & 11594739.669 \\
\hline 0.018 & 0.028 & -1.093 & 0.523 & 67 & 12 & 298.846 & 135155240.559 & 135155739.403 \\
\hline 0.021 & 0.033 & -0.363 & 0.542 & 65 & 11 & 299.439 & 11593115.541 & 11593614.980 \\
\hline 0.021 & 0.036 & -1.886 & 1.245 & 66 & 32 & 298.883 & 135141910.642 & 13514249.523 \\
\hline 0.026 & 0.030 & -0.614 & 1.024 & 67 & 11 & 299.499 & 135154740.226 & 135155239.723 \\
\hline 0.029 & 0.048 & -2.100 & 1.767 & 65 & 12 & 299.224 & 11593615.822 & 11594115.046 \\
\hline 0.030 & 0.047 & -1.368 & 0.877 & 66 & 12 & 297.491 & 135135345.999 & 135135843.489 \\
\hline 0.046 & 0.060 & -1.650 & 1.417 & 72 & 32 & 261.823 & 1381746.242 & 13817828.064 \\
\hline 0.046 & 0.039 & -0.858 & 0.208 & 67 & 31 & 299.288 & 1351611.492 & 135161559.778 \\
\hline 0.047 & 0.045 & -1.305 & 1.540 & 66 & 31 & 299.449 & 135141410.599 & 135141910.046 \\
\hline 0.056 & 0.069 & -2.082 & 1.488 & 72 & 41 & 298.986 & 13817930.796 & 138171429.779 \\
\hline 0.069 & 0.041 & -0.544 & 0.385 & 67 & 32 & 299.149 & $1351616 \quad .526$ & 135162059.672 \\
\hline 0.073 & 0.092 & -1.621 & 1.025 & $\overline{71}$ & $\overline{42}$ & 287.025 & 138154746.530 & 138155233.554 \\
\hline 0.077 & 0.064 & -1.184 & 0.667 & 72 & 11 & 299.750 & 138163330.441 & 138163830.191 \\
\hline 0.078 & 0.052 & -1.272 & 1.020 & 66 & 11 & 299.144 & 135134840.309 & 135135339.449 \\
\hline 0.085 & 0.087 & -2.131 & 1.911 & 71 & 21 & 299.176 & 138152015.474 & 138152514.650 \\
\hline 0.086 & 0.078 & -0.979 & 1.130 & 71 & 32 & 299.142 & 138153610.629 & 13815419.770 \\
\hline 0.087 & 0.090 & -0.973 & 1.631 & 72 & 22 & 299.146 & 138165122.508 & 138165621.652 \\
\hline 0.087 & 0.073 & -1.947 & 0.970 & 72 & 21 & 298.848 & 138164622.829 & 138165121.675 \\
\hline 0.087 & 0.061 & -1.476 & 1.341 & 71 & 12 & 299.574 & 138151410.641 & 138151910.211 \\
\hline 0.091 & 0.070 & -1.558 & 1.578 & 72 & 12 & 299.134 & 138163830.898 & 138164330.029 \\
\hline 0.093 & 0.059 & -1.646 & 1.998 & 71 & 11 & 299.566 & 13815910.495 & 138151410.059 \\
\hline 0.094 & 0.063 & -1.461 & 1.397 & 72 & 31 & 299.134 & $1381659 \quad .620$ & 13817359.754 \\
\hline 0.102 & 0.060 & -0.094 & 0.363 & 68 & 12 & 295.419 & 135171233.107 & 135171728.524 \\
\hline 0.103 & 0.071 & -1.765 & 1.422 & 71 & 31 & 298.721 & 138153110.531 & 13815369.250 \\
\hline 0.106 & 0.052 & -0.823 & 0.918 & 68 & 21 & 359.543 & 135171916.509 & 135172516.050 \\
\hline 0.109 & 0.067 & -0.929 & 1.154 & 71 & 41 & 299.130 & 138154240.824 & 138154739.951 \\
\hline 0.109 & 0.048 & -0.608 & 0.841 & 68 & 11 & 299.142 & 13517728.499 & 135171227.639 \\
\hline 0.114 & 0.056 & -1.836 & 1.645 & 68 & 22 & 358.295 & 135172522.344 & 135173120.639 \\
\hline
\end{tabular}




\begin{tabular}{|c|c|c|c|c|c|c|c|c|}
\hline $6 t 0^{\circ} 6 \varepsilon \varepsilon \subseteq \subseteq I S E I$ & $60 \varepsilon^{\circ} 0+8+\varepsilon I S \varepsilon I$ & $t+1.662$ & II & 99 & $\nabla \varsigma \varepsilon 0$ & S9:०0- & $690^{\circ} 0$ & 0 otI0 \\
\hline $680^{\circ} \varepsilon+8 S \subseteq I S E I$ & $666^{\circ}$ St ES EI SEI & $160^{\circ} \angle 62$ & $2 I$ & 99 & $6 \mathrm{~L} \varepsilon 0$ & $88 \varepsilon^{\circ} 0^{-}$ & $s+0^{\circ} 0$ & SZI0 \\
\hline ZL9.6S 0Z9I SEI & 9ZS 9I 9I SEL & $6+15662$ & $\tau \varepsilon$ & $\angle 9$ & $8 \mathrm{IE:0}$ & $060^{\circ} 0^{-}$ & $\varepsilon S 0^{\circ} 0$ & $9 I^{\circ} 0$ \\
\hline t06.6E IS II ESI & 9SL:0t $9 t$ II $\varepsilon S I$ & ISI' 66 T & I2 & $\varepsilon L$ & $8 \varepsilon 9^{\circ} 0$ & $t S 0^{\circ} 0^{-}$ & $\varepsilon \neq 0^{\circ} 0$ & $9 I^{\circ} 0$ \\
\hline IOI'OS SD II ESI & t60.0S 0t II ESI & $800^{\circ} 00 \varepsilon$ & ZI & $\varepsilon L$ & $79 \varepsilon^{\circ} 0$ & $\mathrm{IZI}^{-} 0^{-}$ & $\angle S 0^{\circ} 0$ & OII0 \\
\hline toS 6 t ot II ESI & S9L0 OS SE I I ESI & $\tau+L 862$ & II & $\varepsilon L$ & $105^{\circ} 0$ & $680^{\circ} 0^{-}$ & $9 \angle 0^{\circ} 0$ & t0I0 \\
\hline 8LL'6S SI 9I SEI & Z6" II 9I SEI & $88 \tau^{\circ} 66 \tau$ & $\mathrm{I \varepsilon}$ & $\angle 9$ & $20 \varepsilon^{\circ} 0$ & $\varepsilon \angle 0^{\circ} 0^{-}$ & $\tau S 0^{\circ} 0$ & $860^{\circ} 0$ \\
\hline 6S000I tI SI $8 E I$ & S66t.0I 6 SI $8 E I$ & $99 S^{\circ} 662$ & II & $\mathrm{IL}$ & $\$ 88^{\circ} 0$ & $68 \mathrm{Z}^{\circ} \mathrm{I}^{-}$ & $\angle L 0^{\circ} 0$ & $\angle 80^{\circ} 0$ \\
\hline tSL'6S E LI 8EI & $0 Z 9^{\circ} \quad 6 \subseteq 918 E I$ & $\neq \varepsilon I .662$ & IE. & $Z L$ & $\nabla \tau \not \nabla^{\circ} 0$ & $2 \mathrm{II}^{\circ} 0^{-}$ & $680^{\circ} 0$ & $\angle 80^{\circ} 0$ \\
\hline IIZ゙0I 6I SI 8EI & It9.0I t & $t \angle S: 662$ & ZI & $I L$ & $\tau S t^{\circ} 0$ & $26 \mathrm{I}^{\circ} \mathrm{I}^{-}$ & $080^{\circ} 0$ & $980^{\circ} 0$ \\
\hline 0S0 91 I SZ LI SEI & 60S'9I 6I LI SEI & $\varepsilon t \varsigma .6 \varsigma \varepsilon$ & I2 & 89 & SSTO 0 & $6 \mathrm{ZI}^{-} 0^{-}$ & $\$ 80^{\circ} 0$ & $\$ 80^{\circ} 0$ \\
\hline $6 \varepsilon 9^{\circ} L Z Z I L I S E I$ & $66 t^{\circ} 8 Z \angle L I S E I$ & $z+1.66 \tau$ & II & 89 & $\$ 29^{\circ} I$ & 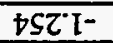 & $080^{\circ} 0$ & $780^{\circ} 0$ \\
\hline$\varepsilon Z L^{\circ} 6 \varepsilon Z S$ SI SEI & $9 z Z^{\circ} 0 t \angle t S I S \varepsilon I$ & $66+662$ & II & $\angle 9$ & $S 6 \varepsilon^{\circ} 0$ & $8 \angle 9^{\circ} 0^{-}$ & sto 0 & $\$ 80^{\circ} 0$ \\
\hline I6I $0 E$ 8E 9I $8 E I$ & I & $0 S L 662$ & II & $\tau L$ & $\angle S D^{\circ} 0$ & $528^{\circ} 0^{-}$ & $080^{\circ} 0$ & $280^{\circ} 0$ \\
\hline $900^{\circ} 0 \mathrm{I} 6 \mathrm{I} \mathrm{tI} \subseteq \varepsilon \mathrm{I}$ & $66 S^{\circ} 0 I$ t I tI SEI & $6 t+662$ & IE & 99 & $\varepsilon+\tau^{\circ} 0$ & $s+20^{-}$ & $570^{\circ} 0$ & $6 \angle 0^{\circ} 0$ \\
\hline $6 \varepsilon 9^{\circ} 0 Z$ IE $\angle I \subseteq E I$ & 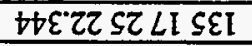 & $S 6 \tau^{\circ} 8 S \varepsilon$ & 22 & 89 & $I \varepsilon \rightarrow 0$ & $\angle+0^{\circ} I^{-}$ & $980^{\circ} 0$ & $\nabla \angle 0^{\circ} 0$ \\
\hline $9+0^{\circ} \mathrm{SI} I+6 \mathrm{SIL}$ & $228^{\circ}$ SI $9 E 6$ SII & \pm 27662 & ZI & $\$ 9$ & s920 & $586^{\circ} 0^{-}$ & $t S 0^{\circ} 0$ & $E L 0^{\circ} 0$ \\
\hline$t Z \varsigma^{\circ} 8 \tau L I L I S E I$ & LOI'EE ZI LI SEI & $6 \mathrm{~L}+56 \mathrm{~S}$ & ZI & 89 & IIt*0 & ZE!० $0^{-}$ & $\$ 80^{\circ} 0$ & $E L 0^{\circ} 0$ \\
\hline$\varepsilon 0 t^{\circ} 6 \varepsilon L S$ SI SEI & 6SS"0t ZS SI SEI & $9+8.862$ & ZI & $\angle 9$ & $692^{\circ} 0$ & $\mathrm{I} \angle b^{\circ} 0^{-}$ & $\varepsilon+0^{\circ} 0$ & $2 \angle 0^{\circ} 0$ \\
\hline $620^{\circ} 0 \varepsilon \varepsilon+918 \varepsilon I$ & $868^{\circ} 0 \varepsilon 8 \varepsilon 9 I 8 \varepsilon I$ & $t \varepsilon I^{\circ} 662$ & ZI & $Z L$ & $680^{\circ} 0$ & $62 t^{\circ} 0^{-}$ & $\angle 80^{\circ} 0$ & $\mathrm{I} \angle 0^{\circ} 0$ \\
\hline $6 \angle L^{\circ} 6 Z$ DI LI $8 E I$ & $96 L^{\circ} 0 E 6 \quad \angle I 8 E I$ & 986862 & It & $\tau L$ & $+9+0$ & $859^{\circ} 0^{-}$ & $S \angle 0^{\circ} 0$ & 6900 \\
\hline $790^{\circ} 8 Z 8 \mathrm{LI} 8 E I$ & $2+2{ }^{\prime} 9+\angle I 8 E I$ & $\varepsilon Z 8^{\circ}[9 Z$ & $Z \varepsilon$ & $Z L$ & $\varepsilon L \varepsilon 0$ & $\varepsilon L E O^{-}$ & $890^{\circ} 0$ & $890^{\circ} 0$ \\
\hline$\varepsilon Z \varsigma^{\prime} 6 \triangleright Z \downarrow I \subseteq \varepsilon I$ & Z†9.0I 6I tI SEI & $\varepsilon 88^{\circ} 86 \tau$ & $Z \varepsilon$ & 99 & $\varepsilon I Z 0$ & $910^{\circ} 0^{-}$ & It000 & $790^{\circ} 0$ \\
\hline $086^{\circ}+19 \varepsilon 6$ SII & $I t S^{\circ} S I I E G$ SII & $6 \varepsilon t ; 662$ & II & 59 & $S L L O 0$ & $69 \varepsilon^{\circ} 0^{-}$ & $\mathrm{IS0^{ \circ } 0}$ & {$\left[90^{\circ} 0\right.$} \\
\hline $699^{\circ} 6 \varepsilon \angle t 6$ SII & 9 IÉ0巾 Zt 6 SII & SSE $66 Z$ & II & $\$ 9$ & $\tau I \tau \cdot 0$ & $760^{\circ} 0^{-}$ & $\varepsilon \neq 0^{\circ} 0$ & $6 \$ 0^{\circ} 0$ \\
\hline$S \angle 9^{\circ}$ IZ IS 9I $8 \varepsilon I$ & $678^{\circ} 279+918 \varepsilon 1$ & 878.862 & IZ & $Z L$ & $\angle 9 D^{\circ} 0$ & $\angle \angle 9^{\circ} O^{-}$ & $780^{\circ} 0$ & $650^{\circ} 0$ \\
\hline I0S"6I $6 \mathrm{I} \mathrm{LI} 8 E \mathrm{I}$ & $\angle T^{\circ} O E+I \angle I \quad 8 E I$ & $950^{\circ} 682$ & $2 t$ & $\tau L$ & $8+200$ & $6920^{-}$ & $0 S 0^{\circ} 0$ & $650^{\circ} 0$ \\
\hline $0 S Z^{\circ} 69 \varepsilon$ SI $8 E I$ & IES 0I IE SI $8 E I$ & IZL:86Z & $I \varepsilon$ & IL & $896^{\circ} 0$ & SSC' I- & $680^{\circ} 0$ & $850^{\circ} 0$ \\
\hline IS6 6E Lt SI 8EI & $\downarrow Z 8^{\circ} 0 \dagger Z \triangleright$ SI $8 E I$ & $0 \varepsilon I^{\circ} 66 Z$ & It & $\mathrm{IL}$ & $t \angle 9^{\circ} 0$ & $t \hbar Z^{*} I^{-}$ & $160^{\circ} 0$ & $850^{\circ} 0$ \\
\hline $\mathrm{I} 66^{\circ} 6 \varepsilon \angle \mathrm{tI}$ SEI & $6 S L^{\circ} 0+\tau+I S E I$ & $\nabla \varepsilon Z^{\circ} 662$ & $I 2$ & 99 & $881^{\circ} 0$ & $9 Z L^{\circ} 0^{-}$ & $\varepsilon \varepsilon 0^{\circ} 0$ & $2 S 0^{\circ} 0$ \\
\hline$S \angle 8^{\circ} 6 \varepsilon Z I$ t & IE8.0† $L t I S E I$ & $9+0^{\circ} 662$ & $2 \tau$ & 99 & $0 z z^{\circ} 0$ & $2 \varepsilon 0^{\circ} 0^{-}$ & $8 \varepsilon 0^{\circ} 0$ & $250^{\circ} 0$ \\
\hline 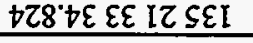 & $69 S^{\circ} S E 8 Z$ IZ SEI & SST 662 & ZI & $O L$ & $\angle 9 I^{\prime} I$ & $960^{\circ} 0^{-}$ & $\angle E O^{\circ} 0$ & $6+0^{\circ} 0$ \\
\hline$\varepsilon 08^{\circ} 6 Z 8 Z$ oZ SEI & $I E L^{\circ} S t+Z 0 Z \subseteq E I$ & $9 \angle 0^{\circ} \nabla z Z$ & $2 \tau$ & 69 & $9 Z L^{\circ} 0$ & 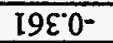 & $0 S 0^{\circ} 0$ & $8+0^{\circ} 0$ \\
\hline tZI'OI tI OI 902 & $889^{\circ} 016$ 0I 902 & $6 \varepsilon t^{\circ} 662$ & II & $S L$ & SItZZ & t020- & $\angle E 0^{\circ} 0$ & $9+0.0$ \\
\hline $2 S 9^{\circ} \mathrm{IZ} 9 S$ 9I $8 E I$ & $80 \varsigma^{\circ} Z Z$ IS 9I $8 E I$ & $9+I^{\circ} 662$ & 22 & $Z L$ & $8 \angle \forall 0^{\circ}$ & $0 \varepsilon S^{\circ} I^{-}$ & I0I.0 & I $¥ 0.0$ \\
\hline ZLZ゙6E 9S II ESI & EtS"9t IS II ESI & $62 L 262$ & $2 z$ & $\varepsilon L$ & $2 \neq 8^{\circ} 0$ & $+t 0^{\circ} 0^{-}$ & $9+0^{\circ} 0$ & $I \neq 0^{\circ} 0$ \\
\hline $0 L L^{\circ} 6$ It SI $8 E I$ & $629^{\circ} 0 I$ 9E SI 8EI & $2+1.662$ & $\tau \varepsilon$ & $\mathrm{IL}$ & $E S I^{\prime} I$ & $0 \varepsilon 8^{\circ} 0^{-}$ & I $60^{\circ} 0$ & $0+0.0$ \\
\hline$L \varepsilon L\llcorner\varepsilon 8 Z$ IZ SEI & LEI SE EZ IZ SEI & $109^{\circ} 662$ & II & $0 L$ & $t$ & $220^{\circ} 0^{-}$ & $620^{\circ} 0$ & $0 E 0^{\circ} 0$ \\
\hline $2 S 6^{6} 6 S \varepsilon \mathrm{I}$ IZ SEI & $\varepsilon 8 \varsigma^{\circ} \varsigma$ t $6 \varepsilon I Z S E I$ & ILËヤS & 22 & $O L$ & $\angle O Z O$ & $\$ 20^{\circ} 0^{-}$ & $8 \varepsilon 0^{\circ} 0$ & 6200 \\
\hline $698^{\circ} 6 \varepsilon$ ot 0Z SEI & Lt9.9t SE OZ SEI & $Z 2 \tau^{\circ} \varepsilon 6 Z$ & $\tau \varepsilon$ & 69 & $\$ 86^{\circ} 0$ & SEI* $0^{-}$ & $0+0^{\circ} 0$ & $620^{\circ} 0$ \\
\hline$\angle S 6^{\circ} 6 Z+91 \mathrm{SEI}$ & tIt $0 E$ 6S SI SEI & sts.662 & IZ & $\angle 9$ & $9 S \varepsilon^{\circ} I$ & $820^{\circ} 0^{-}$ & $\varepsilon \varepsilon 0^{\circ} 0$ & $820^{\circ} 0$ \\
\hline $899^{\circ} 6 \varsigma$ \&Z 0Z SEI & $6 \mapsto I^{\circ} S t 6 I$ D $0 Z$ SEI & IZs" $\supset S Z$ & IZ & 69 & $9 L I^{\circ} 0$ & $620^{\circ} 0^{-}$ & $8 \varepsilon 0^{\circ} 0$ & $\$ 20^{\circ} 0$ \\
\hline $0 S 9^{\circ} \not I S Z S I$ SEI & $t \angle F^{\circ} S I$ OZ SI $8 E I$ & $9 L I^{\circ} 662$ & $\mathfrak{I Z}$ & $\mathrm{IL}$ & 5060 & SEZ $I^{-}$ & $980^{\circ} 0$ & $\$ 20^{\circ} 0$ \\
\hline SIL'6E 6E IZ SEI & $616^{\circ} 0 \triangleright t \varepsilon I Z$ I SEI & $86 L^{\circ} 862$ & IZ & $0 L$ & $2 \mathrm{LI}^{\circ} 0$ & $181^{\circ} 0^{-}$ & $\varepsilon 20^{\circ} 0$ & $\varepsilon Z 0^{\circ} 0$ \\
\hline St6 $6 \varepsilon \subseteq \varepsilon$ OZ SEI & $89 Z^{\circ} 0+0 E 0 Z$ SEI & $6 \angle 9^{\circ} 662$ & $I \varepsilon$ & 69 & $\angle Z b^{\circ} 0$ & $967^{\circ} I^{-}$ & $2 \varepsilon 0^{\circ} 0$ & $\varepsilon Z 0^{\circ} 0$ \\
\hline LES $6 S 9$ ZZ SEI & $8 \mathrm{IL} S Z Z Z \mathrm{SEI}$ & $0 z 8^{\circ} \varepsilon 6 Z$ & $2 t$ & $O L$ & $0 L I^{\circ} 0$ & $8+1^{\circ} 0^{-}$ & $t 20^{\circ} 0$ & $610^{\circ} 0$ \\
\hline$\varepsilon 10^{\circ} \varepsilon \varepsilon 0 \mathrm{I} 90 \tau$ & Soz $8 z$ ol $90 z$ & 218.662 & 12 & $S L$ & $6+8.1$ & $669^{\circ} 0^{-}$ & $6200^{\circ}$ & $810^{\circ} 0$ \\
\hline $90 \varepsilon 6 \mathrm{I} Z \mathrm{ZI} \varepsilon S \mathrm{I}$ & $\neq I \varepsilon^{\prime} 0 Z$ LS II ESI & $966^{\circ} 862$ & $\mathfrak{I \varepsilon}$ & $\varepsilon L$ & $\varepsilon 9 \tau^{\circ} 0$ & $9 \varepsilon 0^{\circ} 0^{-}$ & $9 \varepsilon 0^{\circ} 0$ & $810^{\circ} 0$ \\
\hline ISO $0^{\circ}$ I 6 I 0I 902 & †ZI०OI tI OI 90Z & 626.662 & ZI & $S L$ & SEO I & IS9.0- & $2 \varepsilon 0^{\circ} 0$ & $810^{\circ} 0$ \\
\hline 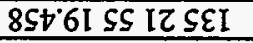 & $\angle O E^{\circ} 0 Z 0 S$ IZ SEI & ISI.662 & $\tau \varepsilon$ & $O L$ & $6 \mathrm{SI}^{\circ} 0$ & $620^{\circ} 0^{-}$ & $620^{\circ} 0$ & 6000 \\
\hline tSS.દE ZS SI 8EI & $0 E S^{\circ} 9 \downarrow \angle t S I$ SEI & $\subseteq 20^{\circ} \angle 8 Z$ & $2 t$ & $\mathrm{I} L$ & SISI & $S \angle Z^{\prime} 0^{-}$ & $\varepsilon 80^{\circ} 0$ & $\angle 00^{\circ} 0$ \\
\hline$S S L^{\circ} 6+\angle I$ ZI ESI & SZZ"0S ZI ZI \&SI & ZES.66Z & $2 t$ & $\varepsilon L$ & ISI:0 & $180^{\circ} 0^{-}$ & 2200 & $700^{\circ} 0$ \\
\hline $0 S L 626$ 9I SEI & I6LOE † 9I SEI & 656862 & $\tau \tau$ & $\angle 9$ & $\angle 80^{\circ} 0$ & $920^{\circ} 0^{-}$ & $\varepsilon I 0^{\circ} 0$ & $\varepsilon 00^{\circ} 0$ \\
\hline $698^{\circ} 6 E$ ZS 6 SII & I0S0t $\angle t 6$ SII & $0 \angle \varepsilon^{\circ} 662$ & $2 z$ & $\$ 9$ & $068^{\circ} 0$ & $920^{\circ} 0^{-}$ & $910^{\circ} 0$ & $\varepsilon 00^{\circ} 0$ \\
\hline $0 L t^{\circ} 6 I$ OS IZ SEI & $\angle 9 I^{\circ} 0 Z$ St IZ SEI & SOE 662 & $\mathfrak{I} \mathcal{E}$ & $0 L$ & $8 \angle 9^{\circ} 0$ & $+\mathrm{IS}^{\circ} 0^{-}$ & $\$ 100$ & $200^{\circ} 0$ \\
\hline $8 \angle \varepsilon^{\prime} 6+Z I$ ZI ESI & $0 \angle 8^{\circ} 0 S \angle$ ZI ESI & 015.862 & It & $\varepsilon L$ & $\angle t I^{\circ} 0$ & $\angle \angle 0^{\circ} 0^{-}$ & $6 \mathrm{~L} 00$ & $\mathrm{I} 00^{\circ} 0$ \\
\hline St8.6S I ZZSEI & $\angle I 9^{\circ} \quad \angle S I Z S E I$ & $0 \varepsilon 2.662$ & It & $O L$ & $\angle E L^{\circ} 0$ & $890^{\circ} 0^{-}$ & $\neq 10^{\circ} 0$ & $100^{\circ} 0$ \\
\hline $969^{\circ} t S \angle E$ OI $90 Z$ & $Z \angle 0^{\circ} \subseteq$ EE OI $90 \tau$ & $9 L S^{\circ} 682$ & $2 z$ & $S L$ & SIt०0 & IE6.0- & $\varepsilon 10^{\circ} 0$ & $100^{\circ} 0^{-}$ \\
\hline $985^{\circ} 6 S_{9}$ ZI ESI & $0 Z 0^{\circ} 9 \mathrm{ZZ} \mathrm{ZI} \mathrm{ESI}$ & $69 S^{\circ} \varepsilon L Z$ & $\tau \varepsilon$ & $\varepsilon L$ & $6 \angle 0^{\circ} 0$ & $165^{\circ} 0^{-}$ & $110^{\circ} 0$ & $200^{\circ} 0^{-}$ \\
\hline
\end{tabular}


720: Tangent Force Coefficient, $\mathrm{Ct}$, at $46.6 \%$ Span

\begin{tabular}{|c|c|c|c|c|c|c|c|c|}
\hline Mean & Std & Min & $\operatorname{Max}$ & Tape & Event & Time Length & Start Time & End Time \\
\hline-0.034 & 0.047 & -1.055 & 1.138 & 71 & 42 & 287.025 & 138154746.530 & 138155233.554 \\
\hline-0.025 & 0.065 & -1.079 & 1.243 & 71 & 41 & 299.130 & 138154240.824 & 138154739.951 \\
\hline-0.024 & 0.065 & -1.092 & 1.475 & 71 & 32 & 299.142 & 138153610.629 & 13815419.770 \\
\hline-0.022 & 0.064 & -0.495 & 0.944 & 68 & 11 & 299.142 & 13517728.499 & 135171227.639 \\
\hline-0.022 & 0.069 & -1.755 & 1.254 & 68 & 22 & 358.295 & 135172522.344 & 135173120.639 \\
\hline-0.020 & 0.067 & -1.208 & 1.220 & 71 & 21 & 299.176 & 138152015.474 & 138152514.650 \\
\hline-0.018 & 0.071 & -1.003 & 1.315 & 71 & 31 & 298.721 & 138153110.531 & 13815369.250 \\
\hline-0.016 & 0.073 & -0.553 & 1.031 & 72 & 22 & 299.146 & 138165122.508 & 138165621.652 \\
\hline-0.012 & 0.076 & -0.302 & 0.460 & 68 & 12 & 295.419 & 135171233.107 & 135171728.524 \\
\hline-0.011 & 0.081 & -0.503 & 0.752 & 68 & 21 & 359.543 & 135171916.509 & 135172516.050 \\
\hline-0.007 & 0.078 & -0.188 & 0.419 & 72 & 12 & 299.134 & 138163830.898 & 138164330.029 \\
\hline-0.006 & 0.077 & -0.960 & 0.869 & 73 & 11 & 298.742 & 153113550.765 & 153114049.504 \\
\hline 0.002 & 0.084 & -1.849 & 1.146 & 71 & 11 & 299.566 & 13815910.495 & 138151410.059 \\
\hline 0.005 & 0.085 & -1.451 & 1.345 & 71 & 12 & 299.574 & 138151410.641 & 138151910.211 \\
\hline 0.006 & 0.083 & -0.986 & 0.908 & 66 & 12 & 297.491 & 135135345.999 & 135135843.489 \\
\hline 0.006 & 0.089 & -1.008 & 1.443 & 72 & 21 & 298.848 & 138164622.829 & 138165121.675 \\
\hline 0.008 & 0.029 & -0.126 & 0.388 & 67 & 22 & 298.959 & 13516430.791 & 13516929.750 \\
\hline 0.013 & 0.082 & -1.418 & 1.047 & 72 & 11 & 299.750 & 138163330.441 & 138163830.191 \\
\hline 0.017 & 0.094 & -0.826 & 0.908 & 72 & 31 & 299.134 & 1381659.620 & 13817359.754 \\
\hline 0.017 & 0.037 & -1.284 & 2.009 & 75 & 22 & 289.576 & 20610335.072 & 206103754.646 \\
\hline 0.017 & 0.031 & -0.700 & 0.916 & 73 & 32 & 273.569 & 15312226.020 & 15312659.586 \\
\hline 0.024 & 0.068 & -0.393 & 0.802 & 66 & 11 & 299.144 & 135134840.309 & 135135339.449 \\
\hline 0.025 & 0.044 & 20.754 & 0.272 & 73 & $\overline{42}$ & 299.532 & 153121250.225 & 153121749.755 \\
\hline 0.029 & 0.042 & -0.662 & 0.983 & 65 & 22 & 299.370 & 11594740.501 & 11595239.869 \\
\hline 0.031 & 0.092 & -0.155 & 0.999 & 67 & 32 & 299.149 & $1351616 \quad .526$ & 135162059.672 \\
\hline 0.034 & 0.045 & -0.057 & 0.275 & 73 & 41 & 298.510 & 15312750.870 & $\begin{array}{lllll}153 & 12 & 12 & 49.378\end{array}$ \\
\hline 0.035 & 0.091 & -0.889 & 1.041 & 73 & 12 & 300.008 & 153114050.094 & 153114550.101 \\
\hline 0.041 & 0.038 & -0.746 & 1.306 & 70 & 41 & 299.230 & $\begin{array}{ll}1352157 & .617\end{array}$ & 13522159.845 \\
\hline 0.041 & 0.043 & -0.027 & 0.254 & 70 & 31 & 299.305 & 135214520.167 & 135215019.470 \\
\hline 0.041 & 0.088 & -1.071 & 1.839 & $\overline{72}$ & 41 & 298.986 & 13817930.796 & 138171429.779 \\
\hline 0.043 & 0.052 & -0.084 & 0.300 & 70 & 32 & 299.151 & 135215020.307 & 135215519.458 \\
\hline 0.050 & 0.067 & -1.364 & 0.617 & 69 & 32 & 293.222 & 135203546.647 & 135204039.869 \\
\hline 0.051 & 0.091 & -0.955 & 0.904 & 73 & 21 & 299.151 & 153114640.756 & 153115139.904 \\
\hline 0.055 & 0.086 & -1.642 & 1.851 & 66 & 31 & 299.449 & 135141410.599 & 135141910.046 \\
\hline 0.056 & 0.054 & -1.022 & 0.765 & 73 & 31 & 298.996 & 153115720.314 & 15312219.306 \\
\hline 0.057 & 0.055 & -0.878 & 1.185 & 69 & 21 & 254.521 & 135201945.149 & 135202359.668 \\
\hline 0.058 & 0.085 & -0.168 & 0.465 & 72 & 32 & 261.823 & 1381746.242 & 13817828.064 \\
\hline 0.060 & 0.055 & -1.309 & 1.449 & 75 & 12 & 299.929 & 206101410.124 & 206101910.051 \\
\hline 0.062 & 0.091 & -0.767 & 0.466 & 67 & 31 & 299.288 & $1351611 \quad .492$ & 135161559.778 \\
\hline 0.066 & 0.069 & -0.923 & 0.740 & 69 & 22 & 224.076 & 135202445.731 & 135202829.803 \\
\hline 0.069 & 0.052 & -1.206 & 1.695 & 75 & 21 & 299.812 & $2061028 \quad .205$ & $2061033 \quad .013$ \\
\hline 0.071 & 0.054 & -0.913 & 1.311 & 69 & 31 & 299.679 & 135203040.268 & 135203539.945 \\
\hline 0.074 & 0.056 & -1.235 & 0.730 & 70 & 22 & 254.371 & 135213945.583 & 135214359.952 \\
\hline 0.077 & 0.047 & -0.109 & 0.277 & 70 & 42 & 293.820 & 1352225.718 & 13522659.537 \\
\hline 0.078 & 0.060 & -0.106 & 0.323 & 73 & 22 & 292.729 & 153115146.543 & 153115639.272 \\
\hline 0.078 & 0.071 & -0.865 & 1.250 & 72 & 42 & 289.056 & 138171430.447 & 138171919.501 \\
\hline 0.078 & 0.054 & -0.100 & 0.282 & 67 & 21 & 299.545 & 135155930.414 & 13516429.957 \\
\hline 0.080 & 0.074 & -0.965 & 1.497 & 65 & 12 & 299.224 & 11593615.822 & 11594115.046 \\
\hline 0.081 & 0.066 & -0.475 & 0.655 & 65 & 11 & 299.439 & 11593115.541 & 11593614.980 \\
\hline 0.084 & 0.070 & -0.156 & 0.453 & 66 & 32 & 298.883 & 135141910.642 & 13514249.523 \\
\hline 0.086 & 0.078 & -1.813 & 0.905 & 67 & 11 & 299.499 & 135154740.226 & 135155239.723 \\
\hline 0.086 & 0.043 & -0.188 & 0.575 & 70 & 21 & 298.798 & 135213440.919 & 135213939.715 \\
\hline 0.090 & 0.062 & -1.898 & 1.389 & 66 & 21 & 299.234 & 13514240.759 & 13514739.991 \\
\hline 0.090 & 0.047 & -0.076 & 0.271 & 70 & 11 & 299.601 & 135212335.137 & 135212834.737 \\
\hline 0.091 & 0.059 & -0.551 & 1.691 & 65 & 21 & 299.355 & 11594240.316 & 11594739.669 \\
\hline 0.092 & 0.066 & -1.357 & 1.328 & 66 & 22 & 299.046 & 13514740.831 & $135 \quad 14 \quad 1239.875$ \\
\hline 0.096 & 0.054 & -1.839 & 1.384 & 75 & 11 & 299.439 & 20610910.688 & $2061014 \quad 10.124$ \\
\hline 0.097 & 0.070 & -0.130 & 0.858 & 67 & 12 & 298.846 & 135155240.559 & 135155739.403 \\
\hline 0.098 & 0.058 & -0.215 & 0.287 & 70 & 12 & 299.255 & 135212835.569 & 135213334.824 \\
\hline
\end{tabular}


721: Tangent Force Coefficient, $\mathrm{Ct}$, at $30 \%$ Span

\begin{tabular}{|c|c|c|c|c|c|c|c|c|}
\hline Mean & Std & Min & $\operatorname{Max}$ & Tape & Event & Time Length & Start Time & End Time \\
\hline-0.308 & 0.108 & -1.695 & 3.560 & 66 & 12 & 297.491 & 135135345.999 & 135135843.489 \\
\hline-0.109 & 0.114 & -1.138 & 0.634 & 66 & 11 & 299.144 & 135134840.309 & 135135339.449 \\
\hline 0.007 & 0.052 & -0.116 & 2.203 & 67 & 22 & 298.959 & 13516430.791 & 13516929.750 \\
\hline 0.023 & 0.090 & -1.737 & 2.385 & 71 & 42 & 287.025 & 138154746.530 & 138155233.554 \\
\hline 0.026 & 0.068 & -1.156 & 1.101 & 69 & 32 & 293.222 & 135203546.647 & 135204039.869 \\
\hline 0.027 & 0.063 & -0.962 & 0.300 & 73 & 42 & 299.532 & 153121250.225 & 153121749.755 \\
\hline 0.030 & 0.062 & -4.056 & 3.808 & 75 & 22 & 289.576 & 20610335.072 & 206103754.646 \\
\hline 0.032 & 0.095 & -2.823 & 2.846 & 71 & 21 & 299.176 & 138152015.474 & 138152514.650 \\
\hline 0.032 & 0.059 & -2.005 & 2.966 & 73 & 32 & 273.569 & 15312226.020 & 15312659.586 \\
\hline 0.037 & 0.071 & -3.733 & 2.583 & 65 & 22 & 299.370 & 11594740.501 & 11595239.869 \\
\hline 0.038 & 0.100 & -2.100 & 1.662 & 72 & 22 & 299.146 & 138165122.508 & 138165621.652 \\
\hline 0.041 & 0.097 & -2.100 & 2.594 & 71 & 32 & 299.142 & 138153610.629 & 13815419.770 \\
\hline 0.049 & 0.087 & -2.273 & 1.302 & 67 & 11 & 299.499 & 135154740.226 & 135155239.723 \\
\hline 0.049 & 0.065 & -0.273 & 0.314 & 73 & 41 & 298.510 & 15312750.870 & 153121249.378 \\
\hline 0.053 & 0.089 & -3.317 & 3.663 & 72 & 21 & 298.848 & 138164622.829 & 138165121.675 \\
\hline 0.054 & 0.094 & -2.102 & 3.471 & 73 & 21 & 299.151 & 153114640.756 & 153115139.904 \\
\hline 0.054 & 0.083 & -0.159 & 0.381 & 67 & 12 & 298.846 & 135155240.559 & 135155739.403 \\
\hline 0.056 & 0.096 & -2.649 & 3.099 & 71 & 41 & 299.130 & 138154240.824 & 138154739.951 \\
\hline 0.057 & 0.092 & -1.676 & 1.267 & 68 & 12 & 295.419 & $13517 \quad 1233.107$ & 135171728.524 \\
\hline 0.057 & 0.096 & -1.688 & 2.587 & 71 & 31 & 298.721 & 138153110.531 & 13815369.250 \\
\hline 0.058 & 0.096 & -2.407 & 3.592 & 71 & 12 & 299.574 & 138151410.641 & 138151910.211 \\
\hline 0.058 & 0.078 & -2.630 & 2.561 & 69 & 22 & 224.076 & 135202445.731 & 135202829.803 \\
\hline 0.060 & 0.092 & -0.793 & 0.502 & 67 & 31 & 299.288 & 1351611.492 & 135161559.778 \\
\hline 0.060 & 0.095 & -2.873 & 4.165 & 66 & 31 & 299.449 & 135141410.599 & 135141910.046 \\
\hline 0.062 & 0.070 & -4.101 & 2.551 & 69 & 21 & 254.521 & 135201945.149 & 135202359.668 \\
\hline 0.062 & 0.065 & -1.175 & 0.384 & 70 & 32 & 299.151 & 135215020.307 & 135215519.458 \\
\hline 0.062 & 0.102 & -2.987 & 3.149 & 72 & 31 & 299.134 & 1381659.620 & 13817359.754 \\
\hline 0.063 & 0.094 & -0.265 & 0.553 & 72 & 12 & 299.134 & 138163830.898 & 138164330.029 \\
\hline 0.063 & 0.088 & -0.194 & 0.798 & $\overline{67}$ & 32 & 299.149 & 1351616.526 & 135162059.672 \\
\hline 0.066 & 0.092 & -2.009 & 3.689 & 68 & 22 & 358.295 & 135172522.344 & 135173120.639 \\
\hline 0.068 & 0.096 & -3.342 & 5.434 & 72 & 11 & 299.750 & 138163330.441 & 138163830.191 \\
\hline 0.069 & 0.096 & -1.579 & 4.633 & 71 & 11 & 299.566 & 13815910.495 & 138151410.059 \\
\hline 0.069 & 0.073 & -2.330 & 6.650 & 75 & 12 & 299.929 & 206101410.124 & 206101910.051 \\
\hline 0.069 & 0.093 & -0.550 & 0.715 & 68 & 21 & 359.543 & 135171916.509 & 135172516.050 \\
\hline 0.069 & 0.088 & -2.609 & 4.517 & 72 & 42 & 289.056 & 138171430.447 & 138171919.501 \\
\hline 0.069 & 0.063 & -0.295 & 1.306 & 70 & 31 & 299.305 & 135214520.167 & 135215019.470 \\
\hline 0.071 & 0.082 & -0.483 & 2.772 & 66 & 32 & 298.883 & 135141910.642 & 13514249.523 \\
\hline 0.072 & 0.054 & -4.576 & 2.074 & 70 & 41 & 299.230 & $\begin{array}{ll}1352157 & .617\end{array}$ & 13522159.845 \\
\hline 0.072 & 0.069 & -1.816 & 5.930 & 73 & 31 & 298.996 & 153115720.314 & 15312219.306 \\
\hline 0.073 & 0.081 & -5.033 & 3.586 & 75 & 11 & 299.439 & 20610910.688 & 206101410.124 \\
\hline 0.074 & 0.097 & -4.409 & 3.529 & 73 & 12 & 300.008 & 153114050.094 & 153114550.101 \\
\hline 0.074 & 0.077 & -0.212 & 0.414 & 70 & 12 & 299.255 & 135212835.569 & 135213334.824 \\
\hline 0.076 & 0.072 & -0.179 & 0.432 & 73 & 22 & 292.729 & 153115146.543 & 153115639.272 \\
\hline 0.076 & 0.088 & -0.795 & 1.131 & 68 & 11 & 299.142 & 13517728.499 & 135171227.639 \\
\hline 0.076 & 0.091 & -1.627 & 0.893 & 72 & 32 & 261.823 & 1381746.242 & 13817828.064 \\
\hline 0.077 & 0.091 & -2.287 & 2.172 & 73 & 11 & 298.742 & 153113550.765 & 153114049.504 \\
\hline 0.077 & 0.097 & -3.862 & 3.691 & 72 & 41 & 298.986 & 13817930.796 & 138171429.779 \\
\hline 0.078 & 0.072 & -5.207 & 4.962 & 75 & 21 & 299.812 & 2061028.205 & 2061033.013 \\
\hline 0.078 & 0.070 & -2.748 & 3.200 & 70 & 22 & 254.371 & 135213945.583 & 135214359.952 \\
\hline 0.078 & 0.066 & -0.187 & 0.358 & 67 & 21 & 299.545 & 135155930.414 & 13516429.957 \\
\hline 0.079 & 0.082 & -3.018 & 3.653 & 66 & 22 & 299.046 & 13514740.831 & 135141239.875 \\
\hline 0.079 & 0.075 & -0.212 & 3.821 & 65 & 11 & 299.439 & 11593115.541 & 11593614.980 \\
\hline 0.079 & 0.078 & -2.576 & 2.868 & 65 & 21 & 299.355 & 11594240.316 & 11594739.669 \\
\hline 0.080 & 0.071 & -2.680 & 3.689 & 69 & 31 & 299.679 & 135203040.268 & 135203539.945 \\
\hline 0.084 & 0.087 & -4.487 & 1.057 & 65 & 12 & 299.224 & 11593615.822 & 11594115.046 \\
\hline 0.086 & 0.062 & -0.164 & 0.351 & 70 & 42 & 293.820 & 1352225.718 & 13522659.537 \\
\hline 0.088 & 0.066 & -0.433 & 1.499 & 70 & 11 & 299.601 & 135212335.137 & 135212834.737 \\
\hline 0.089 & 0.087 & -5.739 & 4.615 & 66 & 21 & 299.234 & 13514240.759 & 13514739.991 \\
\hline 0.094 & 0.063 & -0.182 & 2.098 & 70 & 21 & 298.798 & 135213440.919 & 135213939.715 \\
\hline
\end{tabular}


935: Angle of Attack at $82 \%$ Span (deg)

\begin{tabular}{|c|c|c|c|c|c|c|c|c|}
\hline Mean & Std & Min & $\operatorname{Max}$ & Tape & Event & Time Length & Start Time & End Time \\
\hline-2.850 & 1.916 & -10.630 & 7.934 & 67 & 22 & 298.959 & $13516 \quad 430.791$ & 13516929.750 \\
\hline-2.798 & 1.701 & -17.080 & 21.650 & 75 & 22 & 289.576 & 20610335.072 & 206103754.646 \\
\hline-2.275 & 2.350 & -11.560 & 12.270 & 73 & 42 & 299.532 & 153121250.225 & 153121749.755 \\
\hline-2.203 & 1.675 & -12.500 & 7.676 & $\overline{73}$ & 32 & 273.569 & 15312226.020 & 15312659.586 \\
\hline-1.336 & 1.764 & -10.850 & 6.973 & 73 & 41 & 298.510 & 15312750.870 & 153121249.378 \\
\hline-0.763 & 1.714 & -10.330 & 30.860 & 70 & 31 & 299.305 & 135214520.167 & 135215019.470 \\
\hline-0.573 & 3.257 & -13.480 & 16.100 & 69 & 32 & 293.222 & 135203546.647 & 135204039.869 \\
\hline-0.553 & 2.035 & -17.540 & 13.080 & 75 & 21 & 299.812 & $2061028 \quad .205$ & $2061033 \quad .013$ \\
\hline-0.544 & 1.303 & -9.837 & 11.590 & 70 & 41 & 299.230 & $\begin{array}{lll}1352157 & .617\end{array}$ & 13522159.845 \\
\hline-0.427 & 1.931 & -12.970 & 32.580 & 75 & 12 & 299.929 & 206101410.124 & 206101910.051 \\
\hline-0.409 & 2.101 & -11.090 & 10.470 & 70 & 32 & 299.151 & 135215020.307 & 135215519.458 \\
\hline-0.258 & 1.591 & -16.960 & 6.131 & 65 & 22 & 299.370 & 11594740.501 & 11595239.869 \\
\hline-0.240 & 2.090 & -12.790 & 15.430 & 73 & 31 & 298.996 & 153115720.314 & $15312 \quad 219.306$ \\
\hline 0.460 & 2.362 & -12.230 & 13.010 & 69 & 21 & 254.521 & 135201945.149 & 135202359.668 \\
\hline 0.469 & 1.946 & -12.720 & 37.210 & 69 & 31 & 299.679 & 135203040.268 & 135203539.945 \\
\hline 0.610 & 1.655 & -12.560 & 16.980 & 70 & 42 & 293.820 & 1352225.718 & 13522659.537 \\
\hline 0.939 & 1.788 & -11.190 & 38.110 & 67 & 21 & 299.545 & 135155930.414 & 13516429.957 \\
\hline 1.009 & 1.468 & -10.410 & 38.280 & 70 & 21 & 298.798 & 135213440.919 & 135213939.715 \\
\hline 1.055 & 2.060 & -10.810 & 13.300 & 70 & 22 & 254.371 & 135213945.583 & 135214359.952 \\
\hline 1.072 & 2.213 & -9.950 & 30.680 & 73 & 22 & 292.729 & 153115146.543 & 153115639.272 \\
\hline 1.256 & 1.594 & -10.100 & 10.140 & 70 & 11 & 299.601 & 135212335.137 & 135212834.737 \\
\hline 1.277 & 1.871 & -12.360 & 37.990 & 75 & 11 & 299.439 & 20610910.688 & $2061014 \quad 10.124$ \\
\hline 1.539 & 2.715 & -9.394 & 38.030 & 69 & 22 & 224.076 & 135202445.731 & 135202829.803 \\
\hline 2.061 & 1.943 & -8.617 & 11.390 & 70 & 12 & 299.255 & 135212835.569 & 135213334.824 \\
\hline 2.187 & 1.980 & -9.249 & 37.200 & 66 & 21 & 299.234 & 13514240.759 & 13514739.991 \\
\hline 2.588 & 2.641 & -8.548 & 39.040 & 72 & 42 & 289.056 & 138171430.447 & 138171919.501 \\
\hline 2.676 & 2.201 & -8.361 & 13.350 & 66 & 22 & 299.046 & 13514740.831 & 135141239.875 \\
\hline 3.049 & 2.048 & -7.448 & 17.320 & 67 & 12 & 298.846 & 135155240.559 & 135155739.403 \\
\hline 3.089 & 1.981 & -5.310 & 36.340 & 65 & 21 & 299.355 & 11594240.316 & 11594739.669 \\
\hline 3.197 & 2.310 & -7.946 & 15.500 & 66 & 32 & 298.883 & 135141910.642 & 13514249.523 \\
\hline 3.251 & 2.510 & -5.831 & 16.280 & 65 & 11 & 299.439 & 11593115.541 & 11593614.980 \\
\hline 3.577 & 2.155 & -7.623 & 36.300 & 67 & 11 & 299.499 & 135154740.226 & 135155239.723 \\
\hline 3.784 & 2.695 & -4.821 & 21.010 & 65 & 12 & 299.224 & 11593615.822 & 11594115.046 \\
\hline 4.515 & 2.225 & -12.770 & 25.020 & 73 & 21 & 299.151 & 153114640.756 & 153115139.904 \\
\hline 4.557 & 4.824 & -12.210 & 31.130 & 72 & 32 & 261.823 & 1381746.242 & 13817828.064 \\
\hline 4.704 & 2.783 & -8.412 & 23.520 & 67 & 31 & 299.288 & $1351611 \quad .492$ & 135161559.778 \\
\hline 4.894 & 2.962 & -9.322 & 31.610 & 66 & 31 & 299.449 & 135141410.599 & 135141910.046 \\
\hline 5.109 & 2.977 & -8.514 & 28.830 & 73 & 12 & 300.008 & 153114050.094 & 153114550.101 \\
\hline 6.083 & 2.664 & -5.243 & 27.570 & 66 & 12 & 297.491 & 135135345.999 & 135135843.489 \\
\hline 6.215 & 2.741 & -7.995 & 26.970 & 67 & 32 & 299.149 & $1351616 \quad .526$ & 135162059.672 \\
\hline 6.998 & 5.847 & -9.743 & 39.440 & 72 & 41 & 298.986 & 13817930.796 & 138171429.779 \\
\hline 7.114 & 5.418 & -8.514 & 32.710 & 72 & 11 & 299.750 & 138163330.441 & 138163830.191 \\
\hline 7.914 & 3.768 & -15.640 & 30.680 & 73 & 11 & 298.742 & 153113550.765 & 153114049.504 \\
\hline 9.398 & 4.524 & -4.373 & 36.080 & 71 & 11 & 299.566 & 13815910.495 & 138151410.059 \\
\hline 9.439 & 4.900 & -6.757 & 39.610 & 72 & 31 & 299.134 & 1381659.620 & 13817359.754 \\
\hline 9.485 & 3.506 & -4.666 & 27.570 & 66 & 11 & 299.144 & 135134840.309 & 135135339.449 \\
\hline 9.671 & 5.328 & -16.710 & 37.190 & 71 & 12 & 299.574 & 138151410.641 & 138151910.211 \\
\hline 10.150 & 3.826 & -18.650 & 31.700 & 68 & 11 & 299.142 & 13517728.499 & 135171227.639 \\
\hline 10.400 & 4.155 & -4.756 & 36.150 & 68 & 21 & 359.543 & $135 \quad 17 \quad 19 \quad 16.509$ & 135172516.050 \\
\hline 10.770 & 4.867 & -4.260 & 33.790 & 68 & 12 & 295.419 & 135171233.107 & 135171728.524 \\
\hline 11.020 & 6.120 & -6.712 & 38.420 & 72 & 21 & 298.848 & 138164622.829 & 138165121.675 \\
\hline 11,190 & 4.014 & -4.110 & 34.800 & 68 & 22 & 358.295 & 135172522.344 & 135173120.639 \\
\hline 11.190 & 5.923 & -8.617 & 36.310 & 72 & 12 & 299.134 & 138163830.898 & 138164330.029 \\
\hline 12.480 & 5.362 & -8.044 & 36.740 & 71 & 31 & 298.721 & 138153110.531 & 13815369.250 \\
\hline 13.060 & 4.694 & -2.775 & 35.580 & 71 & 41 & 299.130 & 138154240.824 & 138154739.951 \\
\hline 14.320 & 5.848 & -6.519 & 39.610 & 71 & 32 & 299.142 & 138153610.629 & 13815419.770 \\
\hline 15.250 & 6.520 & -5.324 & 39.650 & 71 & 21 & 299.176 & 138152015.474 & 138152514.650 \\
\hline 15.440 & 6.874 & -8.110 & 39.660 & 72 & 22 & 299.146 & 138165122.508 & 138165621.652 \\
\hline 17.480 & 5.442 & -7.995 & 39.470 & 71 & 42 & 287.025 & 138154746.530 & 138155233.554 \\
\hline
\end{tabular}


936: Angle of Attack at 63.3\% Span

\begin{tabular}{|c|c|c|c|c|c|c|c|c|}
\hline Mean & sta & IMIn & $\operatorname{Max}$ & Tape & Event & Time Length & Start Time & End Time \\
\hline-1.737 & 2.462 & -12.000 & 10.130 & 67 & 22 & 298.959 & 13516430.791 & 13516929.750 \\
\hline 0.266 & 2.133 & -20.400 & 15.390 & 75 & 22 & 289.576 & 20610335.072 & 206103754.646 \\
\hline 0.769 & 2.221 & -6.211 & 12.470 & 65 & 22 & 299.370 & 11594740.501 & 11595239.869 \\
\hline 1.118 & 2.260 & -10.170 & 16.680 & 70 & 31 & 299.305 & 135214520.167 & 135215019.470 \\
\hline 1.390 & 1.801 & -13.480 & 33.260 & 70 & 41 & 299.230 & $1352157 \quad .617$ & 13522159.845 \\
\hline 1.466 & 4.454 & -16.130 & 27.760 & 69 & 32 & 293.222 & 135203546.647 & 135204039.869 \\
\hline 1.624 & 2.923 & -9.262 & 18.220 & 70 & 32 & 299.151 & 135215020.307 & 135215519.458 \\
\hline 2.781 & 2.779 & -9.392 & 30.750 & 69 & 31 & 299.679 & 135203040.268 & 135203539.945 \\
\hline 2.843 & 3.318 & -26.980 & 20.840 & 69 & 21 & 254.521 & 135201945.149 & 135202359.668 \\
\hline 3.026 & 2.191 & -8.616 & 21.150 & 70 & 42 & 293.820 & 1352225.718 & 13522659.537 \\
\hline 3.128 & 2.740 & -71.990 & 19.190 & 75 & 12 & 299.929 & 206101410.124 & 206101910.051 \\
\hline 3.166 & 2.654 & -43.790 & 19.890 & 75 & 21 & 299.812 & 2061028.205 & 2061033.013 \\
\hline 3.436 & 2.635 & -21.230 & 31.790 & 67 & 21 & 299.545 & 135155930.414 & 13516429.957 \\
\hline 3.503 & 1.968 & -11.700 & 19.590 & 70 & 21 & 298.798 & 135213440.919 & 135213939.715 \\
\hline 3.649 & 2.931 & -10.430 & 16.530 & 70 & 22 & 254.371 & 135213945.583 & 135214359.952 \\
\hline 3.899 & 2.190 & -10.270 & 18.310 & 70 & 11 & 299.601 & 135212335.137 & 135212834.737 \\
\hline 4.306 & 3.897 & -9.825 & 22.210 & 69 & 22 & 224.076 & 135202445.731 & 135202829.803 \\
\hline 5.073 & 2.790 & -9.025 & 29.070 & 70 & 12 & 299.255 & 135212835.569 & 135213334.824 \\
\hline 5.150 & 2.982 & -8.372 & 30.170 & 66 & 21 & 299.234 & 13514240.759 & 13514739.991 \\
\hline 5.570 & 2.720 & -51.700 & 25.960 & 75 & 11 & 299.439 & 20610910.688 & 206101410.124 \\
\hline 5.583 & 3.859 & -6.463 & 39.640 & 72 & 42 & 289.056 & 138171430.447 & 138171919.501 \\
\hline 5.596 & 2.915 & -9.244 & 23.430 & 65 & 21 & 299.355 & 11594240.316 & 11594739.669 \\
\hline 5.701 & 3.627 & -27.410 & 25.370 & 65 & 11 & 299.439 & 11593115.541 & 11593614.980 \\
\hline 5.826 & 3.233 & -8.116 & 26.040 & 66 & 22 & 299.046 & 13514740.831 & 135141239.875 \\
\hline 6.473 & 3.092 & -13.270 & 31.110 & 67 & 12 & 298.846 & 135155240.559 & 135155739.403 \\
\hline 6.553 & 3.400 & -8.338 & 35.560 & 66 & 32 & 298.883 & 135141910.642 & 13514249.523 \\
\hline 6.622 & 3.973 & -4.541 & 33.140 & 65 & 12 & 299.224 & 11593615.822 & 11594115.046 \\
\hline 7.305 & 3.221 & -13.650 & 28.730 & 67 & 11 & 299.499 & 135154740.226 & 135155239.723 \\
\hline 8.522 & 7.108 & -25.720 & 39.660 & 72 & 32 & 261.823 & 1381746.242 & 13817828.064 \\
\hline 8.944 & 4.192 & -10.110 & 34.700 & 67 & 31 & 299.288 & 1351611.492 & 135161559.778 \\
\hline 9.035 & 4.318 & -5.835 & 34.450 & 66 & 31 & 299.449 & 135141410.599 & 135141910.046 \\
\hline 10.500 & 3.059 & -0.552 & 28.450 & 73 & 32 & 273.569 & 15312226.020 & 15312659.586 \\
\hline 10.610 & 4.337 & -0.751 & 30.230 & 73 & 42 & 299.532 & 153121250.225 & 153121749.755 \\
\hline 10.720 & 4.107 & -7.716 & 35.790 & 66 & 12 & 297.491 & 135135345.999 & 135135843.489 \\
\hline 11.100 & 4.218 & -15.070 & 38.950 & 67 & 32 & 299.149 & $1351616 \quad .526$ & 135162059.672 \\
\hline 11.910 & 8.388 & -5.594 & 39.660 & 72 & 41 & 298.986 & 13817930.796 & 138171429.779 \\
\hline 12.010 & 7.816 & -7.569 & 39.660 & 72 & 11 & 299.750 & 138163330.441 & 138163830.191 \\
\hline 12.150 & 3.632 & 1.432 & 31.660 & 73 & 41 & 298.510 & 15312750.870 & 153121249.378 \\
\hline 14.550 & $4 . \overline{473}$ & 0.728 & 36.310 & 73 & 31 & 298.996 & 153115720.314 & 15312219.306 \\
\hline 15.230 & 6.497 & -3.241 & 39.650 & 71 & 11 & 299.566 & 13815910.495 & 138151410.059 \\
\hline 15.490 & 7.249 & -4.964 & 39.660 & 72 & 31 & 299.134 & 1381659.620 & 13817359.754 \\
\hline 15.510 & 7.290 & -3.253 & 39.660 & 71 & 12 & 299.574 & 138151410.641 & 138151910.211 \\
\hline 15.630 & 5.168 & -6.796 & 39.400 & 66 & 11 & 299.144 & 135134840.309 & 135135339.449 \\
\hline 16.450 & 5.558 & -13.850 & 39.660 & 68 & 11 & 299.142 & 13517728.499 & 135171227.639 \\
\hline 16.880 & 6.183 & -8.235 & 39.660 & 68 & 21 & 359.543 & 135171916.509 & 135172516.050 \\
\hline 17.290 & 4.775 & 3.465 & 39.020 & 73 & 22 & 292.729 & 153115146.543 & 153115639.272 \\
\hline 17.310 & 7.011 & -6.568 & 39.660 & 68 & 12 & 295.419 & 135171233.107 & 135171728.524 \\
\hline 17.390 & 8.490 & -7.766 & 39.660 & 72 & 21 & 298.848 & 138164622.829 & 138165121.675 \\
\hline 17.580 & 7.983 & -4.398 & 39.660 & 72 & 12 & 299.134 & 138163830.898 & 138164330.029 \\
\hline 17.980 & 5.969 & -7.075 & 39.660 & 68 & 22 & 358.295 & 135172522.344 & 135173120.639 \\
\hline 19.320 & 7.427 & -15.580 & 39.660 & 71 & 31 & 298.721 & 138153110.531 & 13815369.250 \\
\hline 20.240 & 6.573 & -11.420 & 39.660 & 71 & 41 & 299.130 & 138154240.824 & 138154739.951 \\
\hline 21.700 & 7.725 & -16.070 & 39.660 & 71 & 32 & 299.142 & 138153610.629 & 13815419.770 \\
\hline 22.840 & 8.437 & -22.230 & 39.660 & 71 & 21 & 299.176 & 138152015.474 & 138152514.650 \\
\hline 23.050 & 8.822 & -12.520 & 39.660 & 72 & 22 & 299.146 & 138165122.508 & 138165621.652 \\
\hline 24.480 & 4.240 & 0.463 & 39.660 & 73 & 21 & 299.151 & 153114640.756 & 153115139.904 \\
\hline 25.360 & 5.565 & 1.432 & 39.660 & 73 & 12 & 300.008 & 153114050.094 & 153114550.101 \\
\hline 25.640 & 6.889 & -28.070 & 39.660 & 71 & 42 & 287.025 & 138154746.530 & 138155233.554 \\
\hline 29.720 & 5.602 & 2.892 & 39.660 & 73 & 11 & 298.742 & 153113550.765 & 153114049.504 \\
\hline
\end{tabular}


937: Angle of Attack at $46.6 \%$ Span (deg)

\begin{tabular}{|c|c|c|c|c|c|c|c|c|}
\hline Mean & Std & Min & $\operatorname{Max}$ & Tape & Event & Time Length & Start Time & End Time \\
\hline-0.816 & 2.183 & -8.908 & 9.758 & 67 & 22 & 298.959 & 13516430.791 & 13516929.750 \\
\hline 1.707 & 2.040 & -7.481 & 38.140 & 70 & 31 & 299.305 & 135214520.167 & 135215019.470 \\
\hline 1.948 & 1.610 & -9.248 & 15.490 & 70 & 41 & 299.230 & $\begin{array}{ll}1352157 & .617\end{array}$ & $13522 \quad 159.845$ \\
\hline 2.072 & 4.014 & -11.210 & 30.650 & 69 & 32 & 293.222 & 135203546.647 & 135204039.869 \\
\hline 2.171 & 2.655 & -6.235 & 15.460 & 70 & 32 & 299.151 & 135215020.307 & 135215519.458 \\
\hline 2.308 & 2.905 & -20.360 & 21.490 & 75 & 22 & 289.576 & 20610335.072 & 206103754.646 \\
\hline 3.239 & 2.516 & -18.930 & 22.230 & 69 & 31 & 299.679 & 135203040.268 & 135203539.945 \\
\hline 3.263 & 3.313 & -5.736 & 20.020 & 65 & 22 & 299.370 & 11594740.501 & 11595239.869 \\
\hline 3.301 & 3.005 & -14.860 & 18.410 & 69 & 21 & 254.521 & 135201945.149 & 135202359.668 \\
\hline 3.455 & 1.981 & -5.666 & 24.570 & 70 & 42 & 293.820 & 1352225.718 & 13522659.537 \\
\hline 3.879 & 2.380 & -6.935 & 25.350 & 67 & 21 & 299.545 & 135155930.414 & 13516429.957 \\
\hline 3.897 & 1.780 & -7.354 & 26.950 & 70 & 21 & 298.798 & 135213440.919 & 135213939.715 \\
\hline 4.027 & 2.663 & $-6: 249$ & 14.670 & 70 & 22 & 254.371 & 135213945.583 & 135214359.952 \\
\hline 4.264 & 1.982 & -6.337 & 16.470 & 70 & 11 & 299.601 & 135212335.137 & 135212834.737 \\
\hline 4.644 & 3.544 & -6.514 & 26.020 & 69 & 22 & 224.076 & 135202445.731 & 135202829.803 \\
\hline 5.346 & 2.523 & -8.890 & 22.090 & 70 & 12 & 299.255 & 135212835.569 & 135213334.824 \\
\hline 5.427 & 2.698 & -5.107 & 24.000 & 66 & 21 & 299.234 & 13514240.759 & 13514739.991 \\
\hline 5.897 & 3.520 & -4.582 & 32.790 & 72 & 42 & 289.056 & 138171430.447 & 138171919.501 \\
\hline 6.012 & 3.797 & -16.810 & 29.710 & 75 & 21 & 299.812 & $2061028 \quad .205$ & $2061033 \quad .013$ \\
\hline 6.047 & 2.924 & -5.391 & 22.090 & 66 & 22 & 299.046 & 13514740.831 & 135141239.875 \\
\hline 6.680 & 2.785 & -8.979 & 28.050 & 67 & 12 & 298.846 & 135155240.559 & 135155739.403 \\
\hline 6.716 & 3.070 & -4.113 & 30.970 & 66 & 32 & 298.883 & 135141910.642 & 13514249.523 \\
\hline 7.442 & 2.886 & -17.470 & 23.840 & 67 & 11 & 299.499 & 135154740.226 & 135155239.723 \\
\hline 7.455 & 3.742 & -25.020 & 39.370 & 75 & 12 & 299.929 & 206101410.124 & 206101910.051 \\
\hline 8.576 & 6.404 & -14.000 & 39.550 & 72 & 32 & 261.823 & 1381746.242 & 13817828.064 \\
\hline 8.933 & 3.744 & -6.603 & 31.050 & 67 & 31 & 299.288 & $1351611 \quad .492$ & 135161559.778 \\
\hline 8.979 & 3.870 & -23.100 & 30.710 & 66 & 31 & 299.449 & 135141410.599 & 135141910.046 \\
\hline 10.160 & 2.701 & 0.508 & 24.820 & 73 & 32 & 273.569 & 15312226.020 & 15312659.586 \\
\hline 10.260 & 3.864 & 0.661 & 27.610 & 73 & 42 & 299.532 & 153121250.225 & 153121749.755 \\
\hline 10.430 & 5.500 & -11.520 & 38.830 & 65 & 11 & 299.439 & 11593115.541 & 11593614.980 \\
\hline 10.510 & 4.719 & -9.303 & 37.200 & 65 & 21 & 299.355 & 11594240.316 & 11594739.669 \\
\hline 10.520 & 3.647 & -4.175 & 31.620 & 66 & 12 & 297.491 & 135135345.999 & 135135843.489 \\
\hline 10.890 & 3.722 & -4.075 & 35.450 & 67 & 32 & 299.149 & $1351616 \quad .526$ & 135162059.672 \\
\hline 11.120 & 3.873 & -6.220 & 38.220 & 75 & 11 & 299.439 & 20610910.688 & 206101410.124 \\
\hline 11.630 & 3.216 & 2.480 & 30.000 & 73 & 41 & 298.510 & 15312750.870 & 153121249.378 \\
\hline 11.660 & 7.604 & -5.081 & 39.550 & 72 & 41 & 298.986 & 13817930.796 & 138171429.779 \\
\hline 11.750 & 7.042 & -5.269 & 39.460 & 72 & 11 & 299.750 & 138163330.441 & 138163830.191 \\
\hline 12.040 & 6.155 & -23.360 & 39.550 & 65 & 12 & 299.224 & 11593615.822 & 11594115.046 \\
\hline 13.790 & 3.971 & 2.172 & 33.500 & 73 & 31 & 298.996 & 153115720.314 & 15312219.306 \\
\hline 14.670 & 5.756 & -10.150 & 39.550 & $\overline{71}$ & 11 & 299.566 & 13815910.495 & 138151410.059 \\
\hline 14.900 & 6.474 & -1.746 & 39.550 & $\overline{72}$ & 31 & 299.134 & 1381659.620 & 13817359.754 \\
\hline 14.930 & 4.525 & -3.815 & 37.930 & 66 & 11 & 299.144 & 135134840.309 & 135135339.449 \\
\hline 14.960 & 6.537 & -22.010 & 39.550 & 71 & 12 & 299.574 & $138 \quad 15 \quad 14 \quad 10.641$ & 138151910.211 \\
\hline 15.680 & 4.859 & -19.500 & 38.780 & 68 & 11 & 299.142 & 13517728.499 & 135171227.639 \\
\hline 16.070 & 5.447 & -12.680 & 39.550 & 68 & 21 & 359.543 & 135171916.509 & 135172516.050 \\
\hline 16.240 & 4.255 & 4.435 & 36.820 & 73 & 22 & 292.729 & 153115146.543 & 153115639.272 \\
\hline 16.450 & 6.201 & -3.803 & 39.550 & 68 & 12 & 295.419 & 135171233.107 & 135171728.524 \\
\hline 16.620 & 7.661 & -36.800 & 39.550 & 72 & 21 & 298.848 & 138164622.829 & 138165121.675 \\
\hline 16.820 & 7.225 & -2.229 & 39.550 & 72 & 12 & 299.134 & 138163830.898 & 138164330.029 \\
\hline 17.040 & 5.244 & -2.930 & 39.550 & 68 & 22 & 358.295 & 135172522.344 & 135173120.639 \\
\hline 18.390 & 6.655 & -20.090 & 39.550 & 71 & 31 & 298.721 & 138153110.531 & 13815369.250 \\
\hline 19.200 & 5.846 & -16.690 & 39.550 & 71 & 41 & 299.130 & 138154240.824 & 138154739.951 \\
\hline 20.550 & 6.945 & -10.960 & 39.550 & 71 & 32 & 299.142 & 138153610.629 & 13815419.770 \\
\hline 21.610 & 7.683 & -36.800 & 39.550 & 71 & 21 & 299.176 & $1381520 \quad 15.474$ & 138152514.650 \\
\hline 21.880 & 8.120 & -3.308 & 39.550 & 72 & 22 & 299.146 & 138165122.508 & 138165621.652 \\
\hline 22.770 & 3.801 & 3.486 & 39.550 & 73 & 21 & 299.151 & 153114640.756 & 153115139.904 \\
\hline 23.650 & 5.108 & 4.774 & 39.550 & 73 & 12 & 300.008 & 153114050.094 & 153114550.101 \\
\hline 24.170 & 6.284 & -32.500 & 39.550 & 71 & $\overline{42}$ & 287.025 & 138154746.530 & 138155233.554 \\
\hline 27.860 & 5.402 & 6.617 & 39.550 & 73 & 11 & 298.742 & 153113550.765 & 153114049.504 \\
\hline
\end{tabular}


938: Angle of Attack at 30\% Span (deg)

Mean Std Min Max Tape Event Time Length

\begin{tabular}{|c|c|c|c|c|c|c|c|c|}
\hline Mean & Sta & Min & Max & Tape & Event & Time Length & Start Time & End Time \\
\hline 3.643 & 5.966 & -20.350 & 39.120 & 67 & 22 & 298.959 & 13516430.791 & 13516929.750 \\
\hline 6.611 & 5.776 & -25.920 & 33.900 & 73 & 32 & 273.569 & 15312226.020 & 15312659.586 \\
\hline 6.643 & 7.721 & -25.920 & 39.660 & 73 & 42 & 299.532 & 153121250.225 & 153121749.755 \\
\hline 6.877 & 5.799 & -11.750 & 34.810 & 65 & 22 & 299.370 & 11594740.501 & 11595239.869 \\
\hline 7.295 & 6.166 & -25.920 & 38.070 & 75 & $\overline{22}$ & 289.576 & 20610335.072 & 206103754.646 \\
\hline 9.199 & 6.663 & -18.920 & 39.170 & 73 & 41 & 298.510 & 15312750.870 & 153121249.378 \\
\hline 10.370 & 6.201 & -23.850 & 39.280 & 70 & 31 & 299.305 & 135214520.167 & 135215019.470 \\
\hline 11.150 & 5.011 & -16.040 & 35.900 & 70 & 41 & 299.230 & 1352157.617 & 13522159.845 \\
\hline 11.600 & 7.873 & -25.920 & 39.660 & 70 & 32 & 299.151 & 135215020.307 & 135215519.458 \\
\hline 11.740 & 11.920 & -25.920 & 39.660 & 69 & 32 & 293.222 & 135203546.647 & 135204039.869 \\
\hline 12.750 & 8.147 & -25.920 & 39.660 & 75 & 12 & 299.929 & 206101410.124 & 206101910.051 \\
\hline 13.370 & 8.047 & -25.920 & 39.660 & 73 & 31 & 298.996 & 153115720.314 & 15312219.306 \\
\hline 13.790 & 7.795 & -25.920 & 39.660 & 75 & 21 & 299.812 & 2061028.205 & 2061033.013 \\
\hline 14.800 & 7.967 & -25.920 & 39.660 & 69 & 31 & 299.679 & 135203040.268 & 135203539.945 \\
\hline 14.810 & 6.761 & -25.920 & 39.660 & 70 & 42 & 293.820 & 1352225.718 & 13522659.537 \\
\hline 15.510 & 9.545 & -25.920 & 39.660 & 69 & 21 & 254.521 & 135201945.149 & 135202359.668 \\
\hline 16.030 & 6.775 & -22.830 & 39.660 & 70 & 21 & 298.798 & 135213440.919 & 135213939.715 \\
\hline 16.380 & 7.025 & -16.410 & 39.640 & 67 & 21 & 299.545 & 135155930.414 & 13516429.957 \\
\hline 16.440 & 7.733 & -21.490 & 39.660 & 70 & 22 & 254.371 & 135213945.583 & 135214359.952 \\
\hline 16.860 & 6.801 & -18.050 & 39.660 & 70 & 11 & 299.601 & 135212335.137 & 135212834.737 \\
\hline 16.910 & 8.372 & -22.350 & 39.660 & 73 & 22 & 292.729 & 153115146.543 & 153115639.272 \\
\hline 17.900 & 9.878 & -21.000 & 39.660 & 69 & 22 & 224.076 & 135202445.731 & 135202829.803 \\
\hline 18.040 & 8.807 & -12.830 & 39.660 & 65 & 11 & 299.439 & 11593115.541 & 11593614.980 \\
\hline 18.510 & 8.072 & -25.760 & 39.660 & 65 & 21 & 299.355 & 11594240.316 & 11594739.669 \\
\hline 18.520 & 8.338 & -25.920 & 39.660 & 75 & 11 & 299.439 & 20610910.688 & 206101410.124 \\
\hline 19.360 & 7.498 & -19.110 & 39.660 & 70 & 12 & 299.255 & 135212835.569 & 135213334.824 \\
\hline 19.770 & 9.742 & -25.680 & 39.660 & 72 & 42 & 289.056 & 138171430.447 & 138171919.501 \\
\hline 19.910 & 8.494 & -25.920 & 39.660 & 66 & 21 & 299.234 & 13514240.759 & 13514739.991 \\
\hline 20.240 & 9.718 & -19.010 & 39.660 & 65 & 12 & 299.224 & 11593615.822 & 11594115.046 \\
\hline 21.060 & 8.053 & -15.630 & 39.660 & 66 & 22 & 299.046 & 13514740.831 & 135141239.875 \\
\hline 22.260 & 8.050 & -25.920 & 39.660 & 66 & 32 & 298.883 & $1351419 \quad 10.642$ & 13514249.523 \\
\hline 22.680 & 7.293 & -25.920 & 39.660 & 67 & 12 & 298.846 & 135155240.559 & 135155739.403 \\
\hline 23.020 & 11.880 & -25.920 & 39.660 & 72 & 32 & 261.823 & 1381746.242 & 13817828.064 \\
\hline 24.760 & 6.742 & -10.870 & 39.660 & 67 & 11 & 299.499 & 135154740.226 & 135155239.723 \\
\hline 26.700 & 8.031 & -6.609 & 39.660 & 66 & 31 & 299.449 & 135141410.599 & 135141910.046 \\
\hline 26.910 & 8.659 & -21.530 & 39.660 & 67 & 31 & 299.288 & $1351611 \quad .492$ & 135161559.778 \\
\hline 27.200 & 7.796 & -12.330 & 39.660 & 73 & 21 & 299.151 & 153114640.756 & 153115139.904 \\
\hline 27.200 & 10.360 & -25.920 & 39.660 & 72 & 41 & 298.986 & 13817930.796 & 138171429.779 \\
\hline 27.830 & 8.932 & -25.920 & 39.660 & 73 & 12 & 300.008 & 153114050.094 & 153114550.101 \\
\hline 28.210 & 11.410 & -20.020 & 39.660 & 72 & 11 & 299.750 & 138163330.441 & 138163830.191 \\
\hline 29.560 & 7.475 & -7.480 & 39.660 & 66 & 12 & 297.491 & 135135345.999 & 135135843.489 \\
\hline 30.490 & 7.103 & -4.492 & 39.660 & 67 & 32 & 299.149 & 1351616.526 & 135162059.672 \\
\hline 32.050 & 8.772 & -25.920 & 39.660 & 72 & 31 & 299.134 & $1381659 \quad .620$ & 13817359.754 \\
\hline 32.450 & 7.365 & -6.237 & 39.660 & 73 & 11 & 298.742 & 153113550.765 & 153114049.504 \\
\hline 33.160 & 7.192 & -3.946 & 39.660 & 71 & 12 & 299.574 & 138151410.641 & 138151910.211 \\
\hline 33.300 & 8.709 & -25.920 & 39.660 & 72 & 21 & 298.848 & 138164622.829 & 138165121.675 \\
\hline 33.450 & 7.032 & -16.300 & 39.660 & 71 & 11 & 299.566 & 13815910.495 & 138151410.059 \\
\hline 33.920 & 7.026 & -9.598 & 39.660 & 72 & 12 & 299.134 & 138163830.898 & 138164330.029 \\
\hline 34.630 & 5.939 & -6.745 & 39.660 & 66 & 11 & 299.144 & 135134840.309 & 135135339.449 \\
\hline 34.680 & 6.423 & -21.920 & 39.660 & 68 & 21 & 359.543 & 135171916.509 & 135172516.050 \\
\hline 34.680 & 7.314 & -25.920 & 39.660 & 68 & 12 & 295.419 & 135171233.107 & 135171728.524 \\
\hline 34.760 & 5.880 & -25.920 & 39.660 & 68 & 11 & 299.142 & 13517728.499 & 135171227.639 \\
\hline 35.250 & 6.885 & -25.920 & 39.660 & 71 & 31 & 298.721 & 138153110.531 & 13815369.250 \\
\hline 35.800 & 5.378 & 0.961 & 39.660 & 68 & 22 & 358.295 & 135172522.344 & 135173120.639 \\
\hline 35.930 & 6.007 & -23.050 & 39.660 & 72 & 22 & 299.146 & 138165122.508 & 138165621.652 \\
\hline 36.250 & 6.090 & -23.130 & 39.660 & 71 & 21 & 299.176 & 138152015.474 & 138152514.650 \\
\hline 36.310 & 5.787 & -25.920 & 39.660 & 71 & 32 & 299.142 & 138153610.629 & 13815419.770 \\
\hline 36.370 & 5.415 & -9.730 & 39.660 & 71 & 41 & 299.130 & 138154240.824 & 138154739.951 \\
\hline 37.680 & 3.882 & -25.920 & 39.660 & 71 & 42 & 287.025 & 138154746.530 & 138155233.554 \\
\hline
\end{tabular}


Appendix B:

CYCCAT.C BEN_IO.C

READ.C

MAIN.H

MAKEFLE

LAST_BIN.DAT 
/* cyccat.c 020492 Cycle Categorization program

This program reads 400-byte, 101 -datum records containing 1 pressure profile. When the new data with 5 profiles is available, a new version will have to be written. The changes will be in the areas:

- expand 'datain $\square$ '

- check read_in_chanls to see if it will handle new file (See pcbins.c for further detail).

This version reads BRE format files - binary 4-byte reals, engineering units. For each cycle of data it computes the mean and standard deviation of specified channels (one cycle consists of one set of data with azimuthal angle from 0 to 360 degrees). The data from specified channels written to an ASCII file, hdr_file.out. This file may be used as-is to plot out data from each cycle. The data is also written to a user file, hdr_file.sum. The following channels are specified:

Wind Velocity (disc av wind speed channel) \#223

Yaw (yaw angle - vpa prop vane direct hub height) $\quad \# 60 \# 47$

Horizontal Shear (3:00-9:00)/ 10.1 (\#40-\#44)/10.1

Vertical Shear $(12: 00-6: 00) / 10.1 \quad(\# 38 \# 42) / 10.1$

WRITTEN: M. Querijero, T. Young SER/WIND */

$I^{*} \quad$ Wind Direction Modifications by Steve Huyer on 10/30/91 */

/* DECLARATION OF GLOBAL VARIABLES */

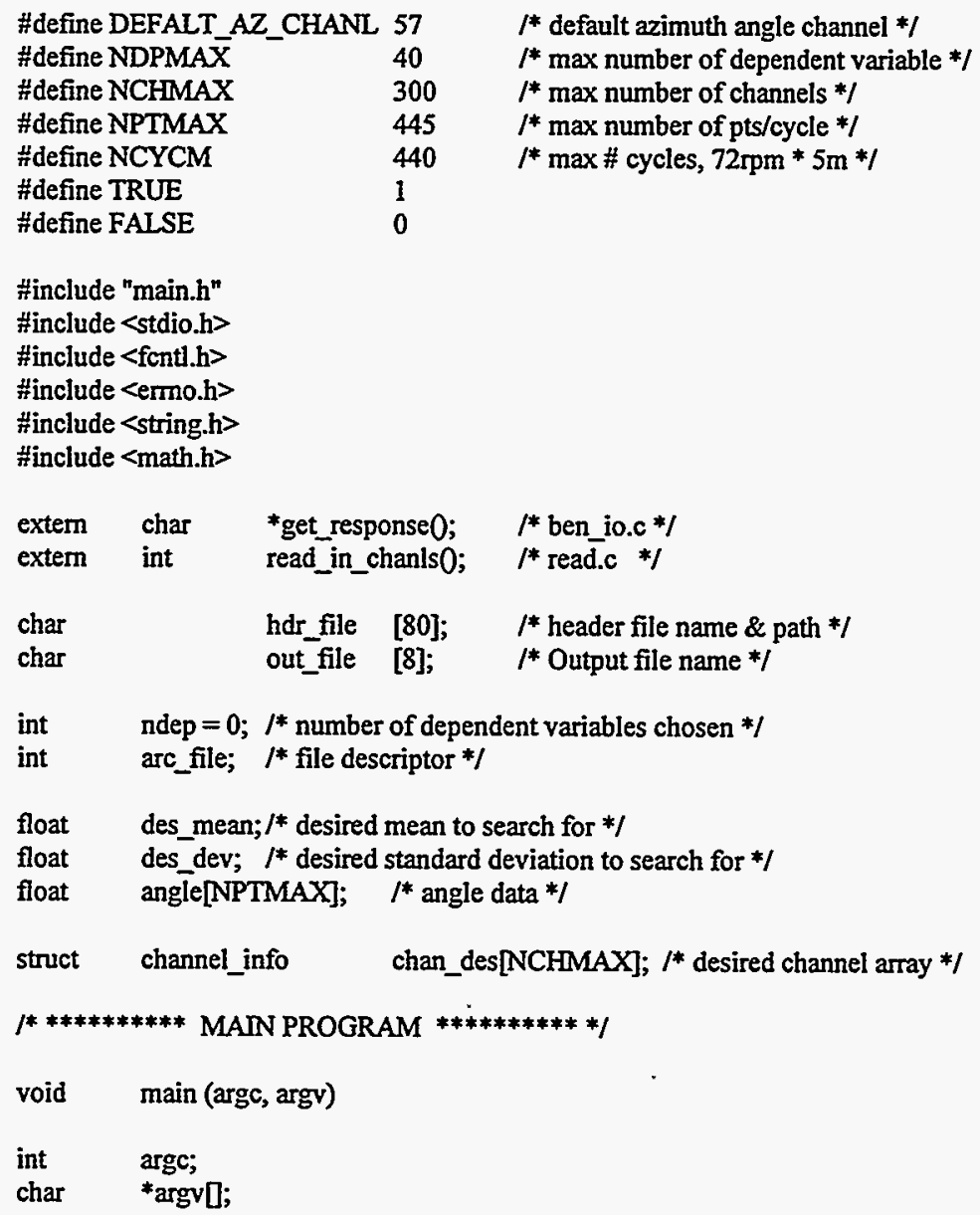

\#define NPTMAX

\#define NCYCM

\#define TRUE

\#define FALSE

I* max number of dependent variable *I

I* max number of channels *

I* max number of pts/cycle *l

1

I* max \# cycles, $72 \mathrm{rpm} * 5 \mathrm{~m} * 1$

\section{\#include "main.h" \\ \#include <stdio.h> \\ \#include $<$ fontl.h $>$ \\ \#include <ermo.h $>$ \\ \#include <string.h> \\ \#include $<$ math.h $>$}

1* MAIN can be run with or without arguments. argv and arge specify whether or not to read a file containing desired channels. */

$\{*$ begin main *

I* VARIABLE DECLARATION - MAIN *

$\begin{array}{lll}\text { int } & \text { start; } & / * \text { start cycle logical */ } \\ \text { int } & \text { npts; } & / * \text { number of points in cycle, temporary */ }\end{array}$




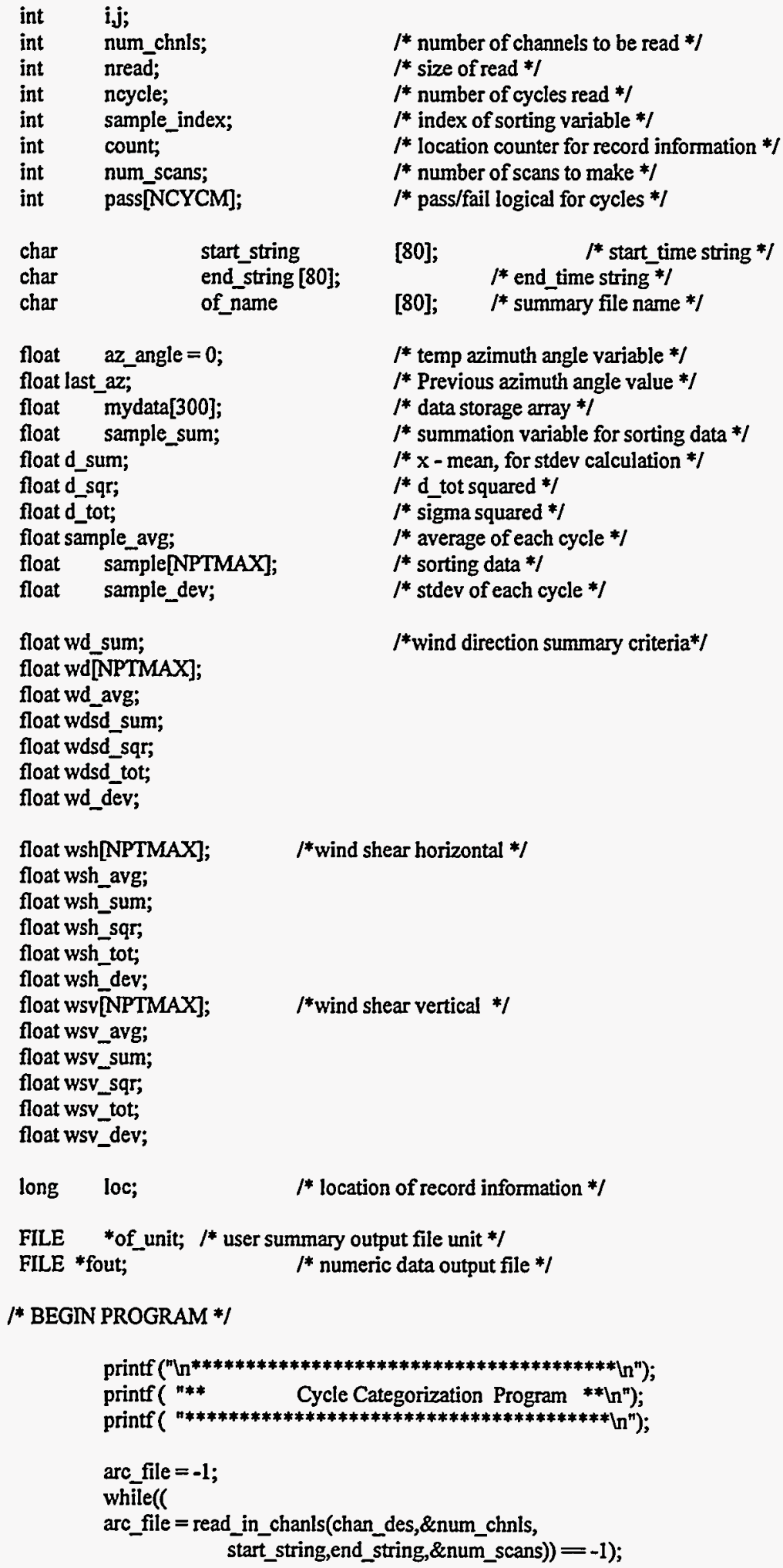

float wd sum;

float wd[NPTMAX];

$I *$ temp azimuth angle variable *f

$/ *$ Previous azimuth angle value *I

$1 *$ data storage array *I

I* summation variable for sorting data *I

$l^{*} \mathrm{x}$ - mean, for stdev calculation $*$ I

$1 *$ d_tot squared *I

$1 *$ sigma squared *I

$1 *$ average of each cycle $* I$

/* sorting data *I

/* stdev of each cycle *I

float wd avg;

float wdsd_sum;

float wdsd sqr;

float wdsd_tot;

float wd dev;

I* get index to sort cycle on; NOTE: assumes a 360 degree cycle */

sample_index =223; /* Disc Av Wind Speed for wind velocity*/

$/ *$ open up summary file * 


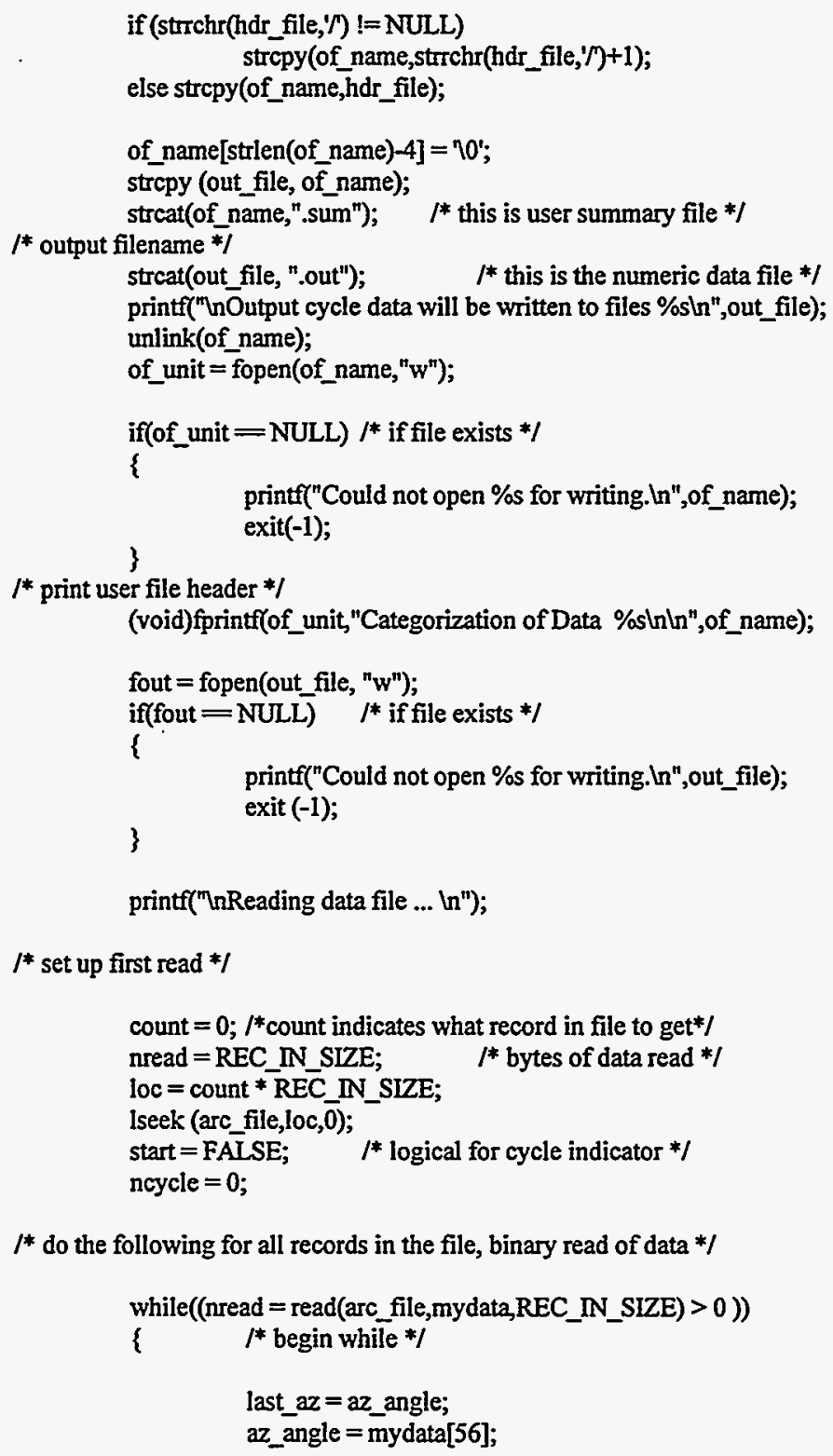

$/ *$ do the following for all records in the file, binary read of data *I

$I^{*}$ this series of if statements tests azimuthal angle to see what cycle it is in, whether to start or finish a cycle, etc. */

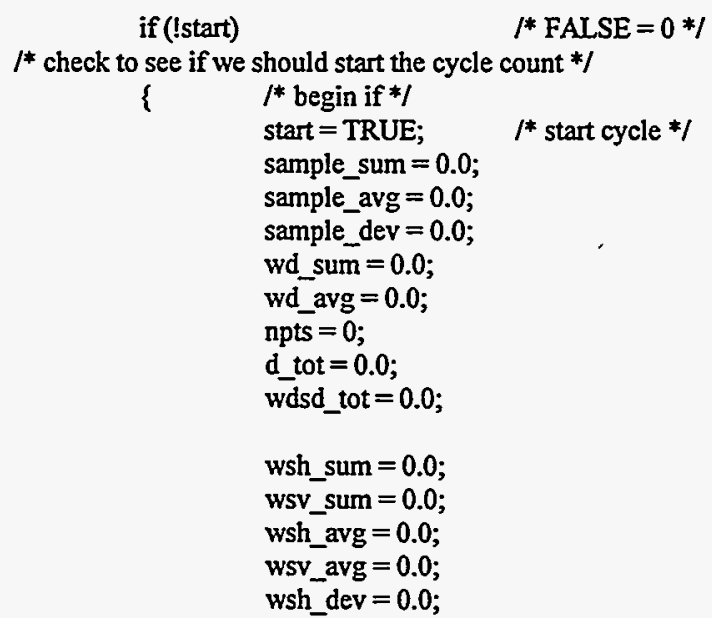




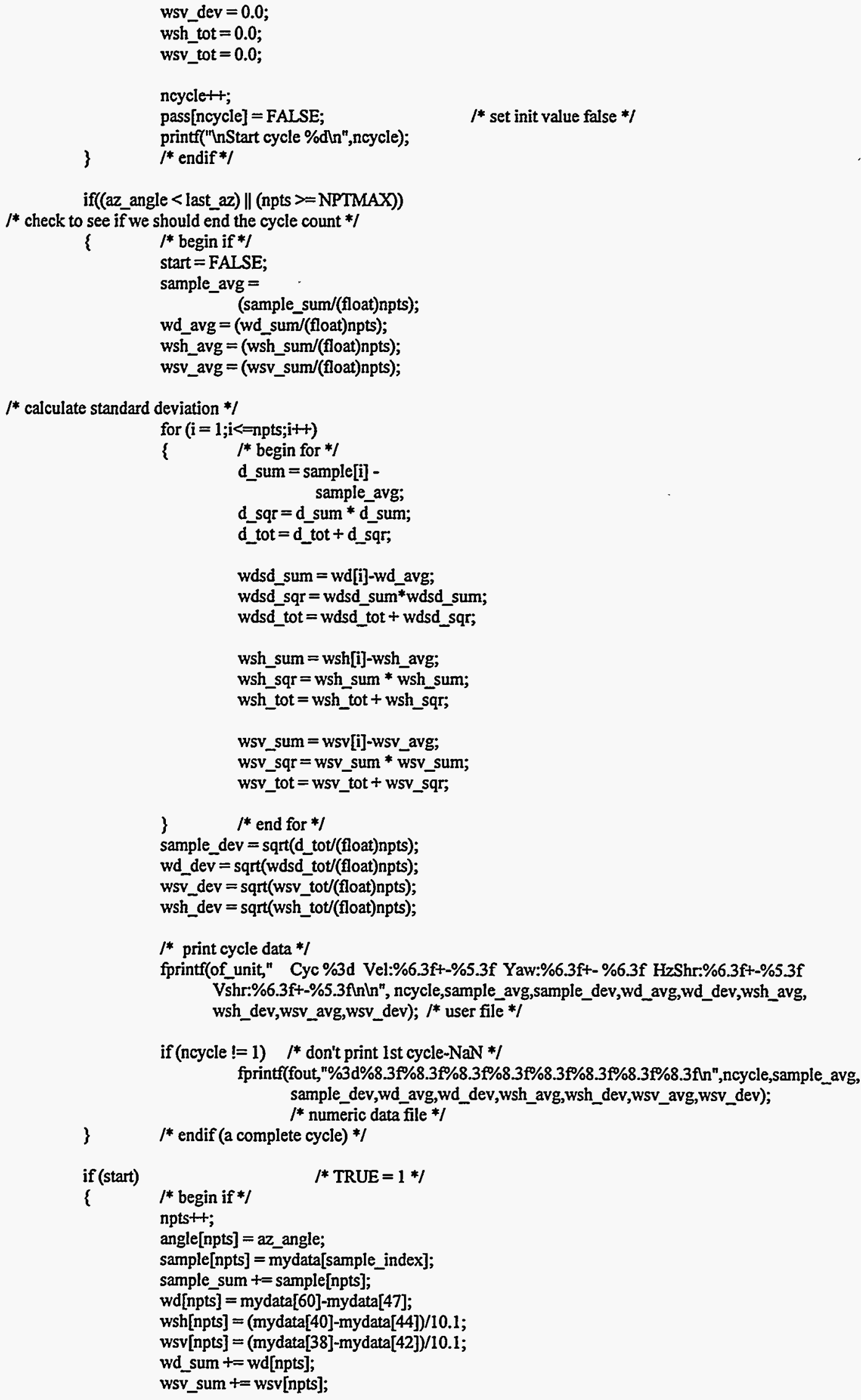




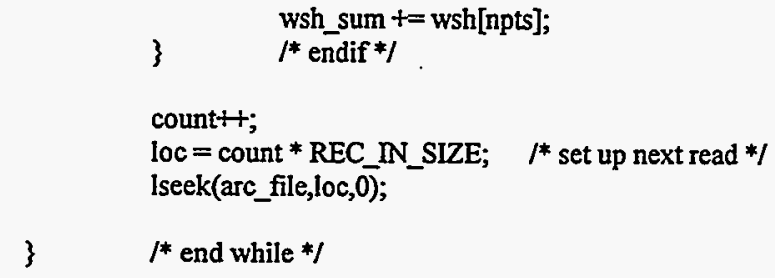




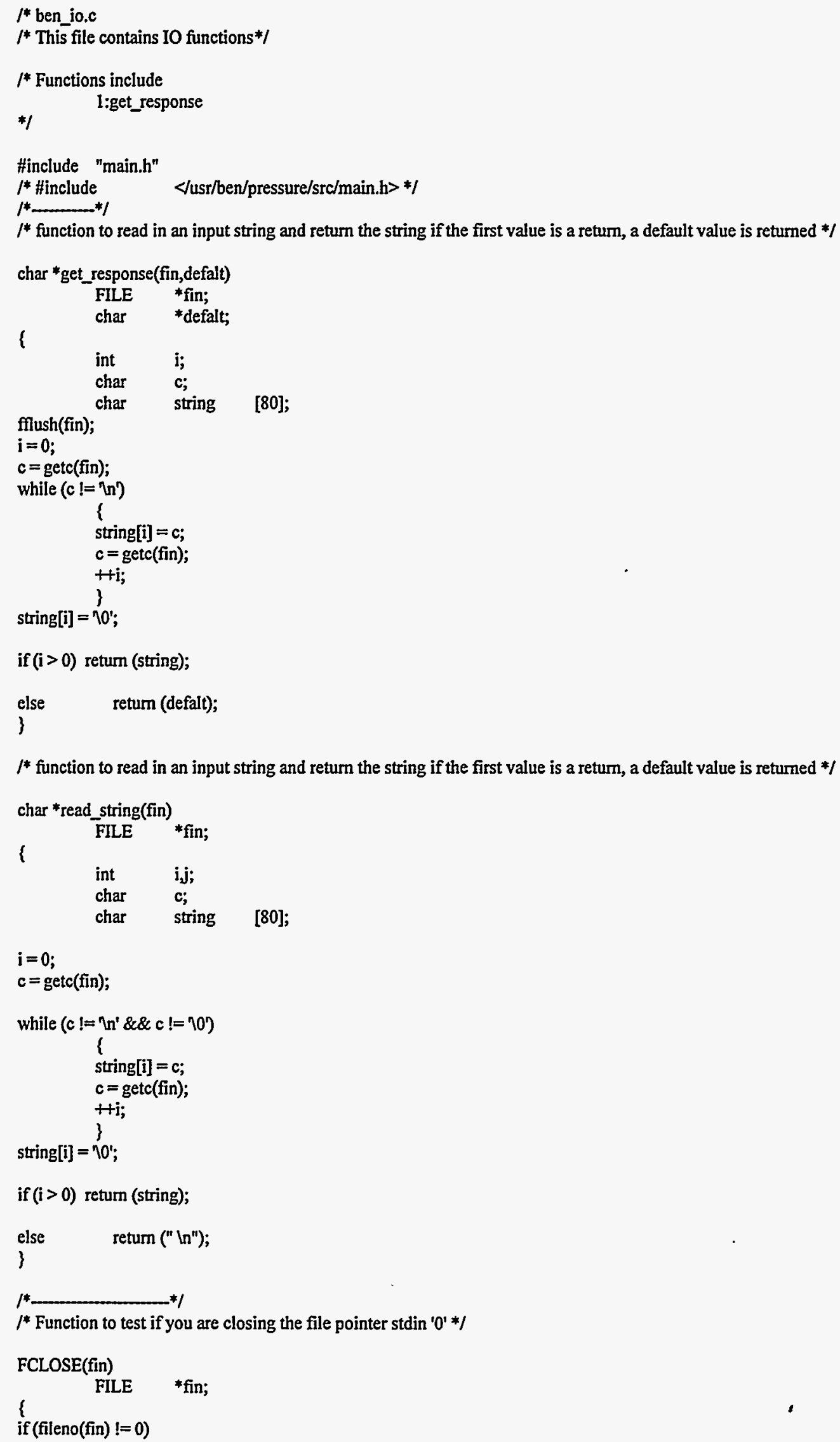


fclose(fin);

return;

\} 


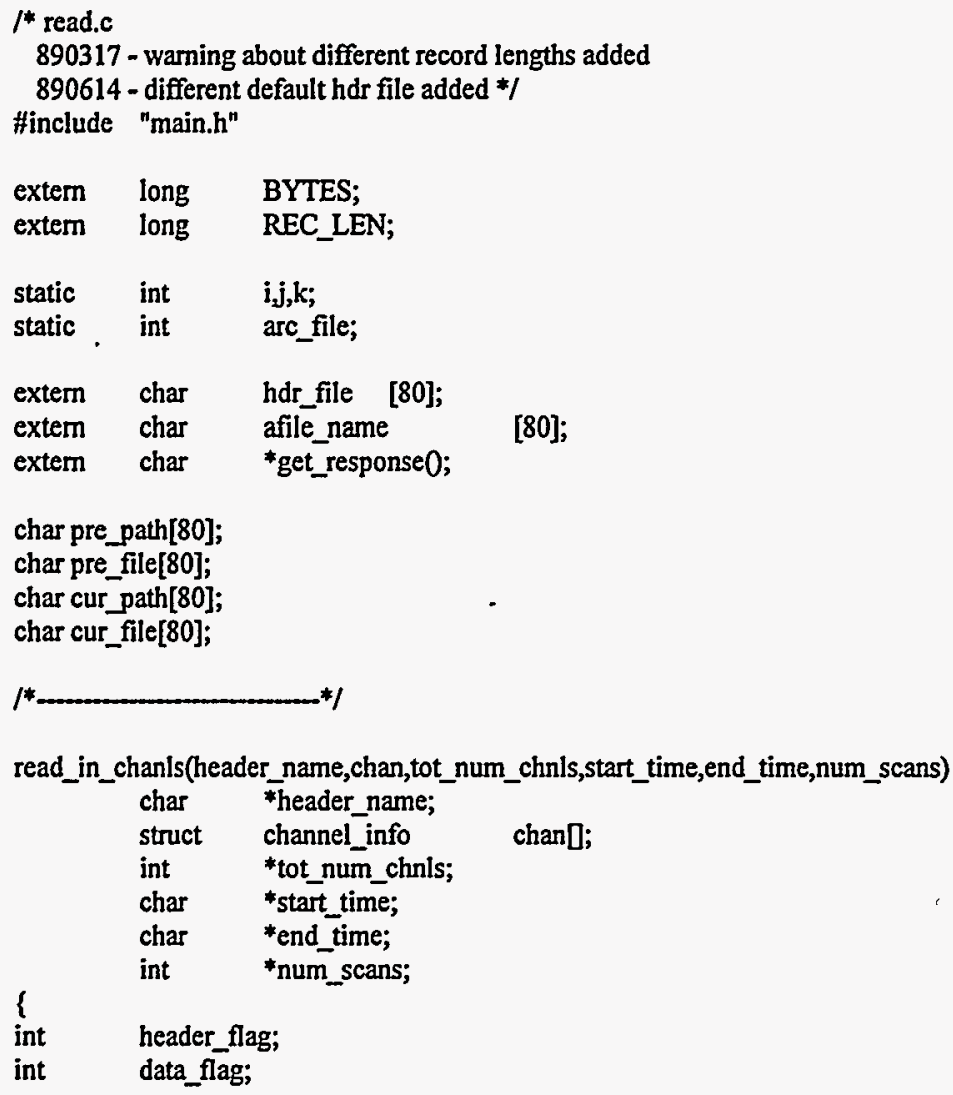

$l^{*}$ check if header file exists *I

header_flag = check_header_file 0 ;

if (header_flag $=-1$ )

return (header_flag);

$l^{*}$ if the header file exists, check if the data file exists *I

data_flag = check_data_file 0 ;

if (data_flag $=-1$ )

return (data_flag);

strcpy(header_name,hdr_file);

$/ *$ read in the header file */

read_header_file(chan,tot_num_chnls,start_time,end_time,num_scans);

retum (arc_file);

\}

l゙\#\#\#\#\#\#\#\#\#\#\#\#\#\#\#\#\#\#\#\#\#\#\#\#\#\#\#\#\#\#\#\#\#\#\#\#\#\#\#\#\#\#\#\#\#\#\#\#\#\#\#\#\#\#\#゙/ prevparO

\{

static char fnam[ = "last_bin.dat";

FILE *fp;

1* Read in parameters from last run */ fp = fopen(fnam, ${ }^{n} "$ );

if(fp != NULL) /* If file exists */

\{

$$
\text { fscanf(fp, "\%s",pre_path); } \quad \text { /* Read previous path and *f }
$$




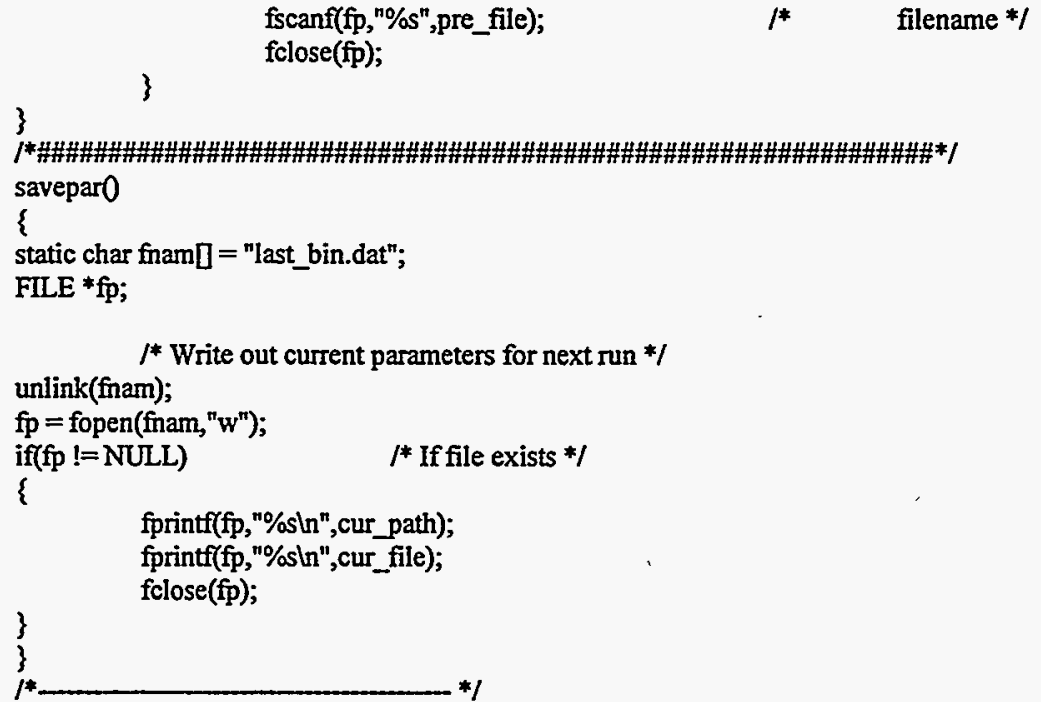

$1^{*}$ This function will test if a header file exists. If it does then it will read the first line for a data format to confirm that its a header file. If the header file doesn't exist the user will be prompted for a new header file *f

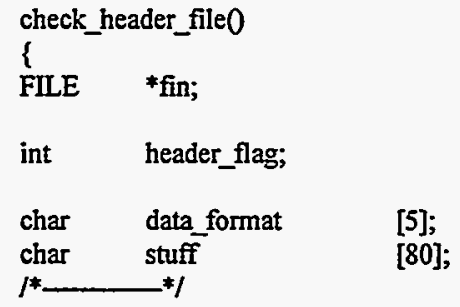

$l^{*}$ if file exists check if its a header file by checking the first line for the data format *

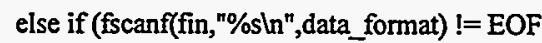

\&\& strcmp("BRR",data_format) != 0 \&\& strcmp("BRE",data_format) !=0

\&\& strcmp("BIR",data_format) != 0 \&\& strcmp("BIE",data_format) !=0)

\{

printf("In!!ERROR!! \%s not a header fileln",hdr_file);

printf("InHit <RETURN> to continue : ");

fflush(stdout);

getc(stdin);

header_flag $=-1$

$3 / *$ else if $*$

$I^{*}$ if the file exists and the first line is a data format, then set the header_flag to 1 for a good file *I 


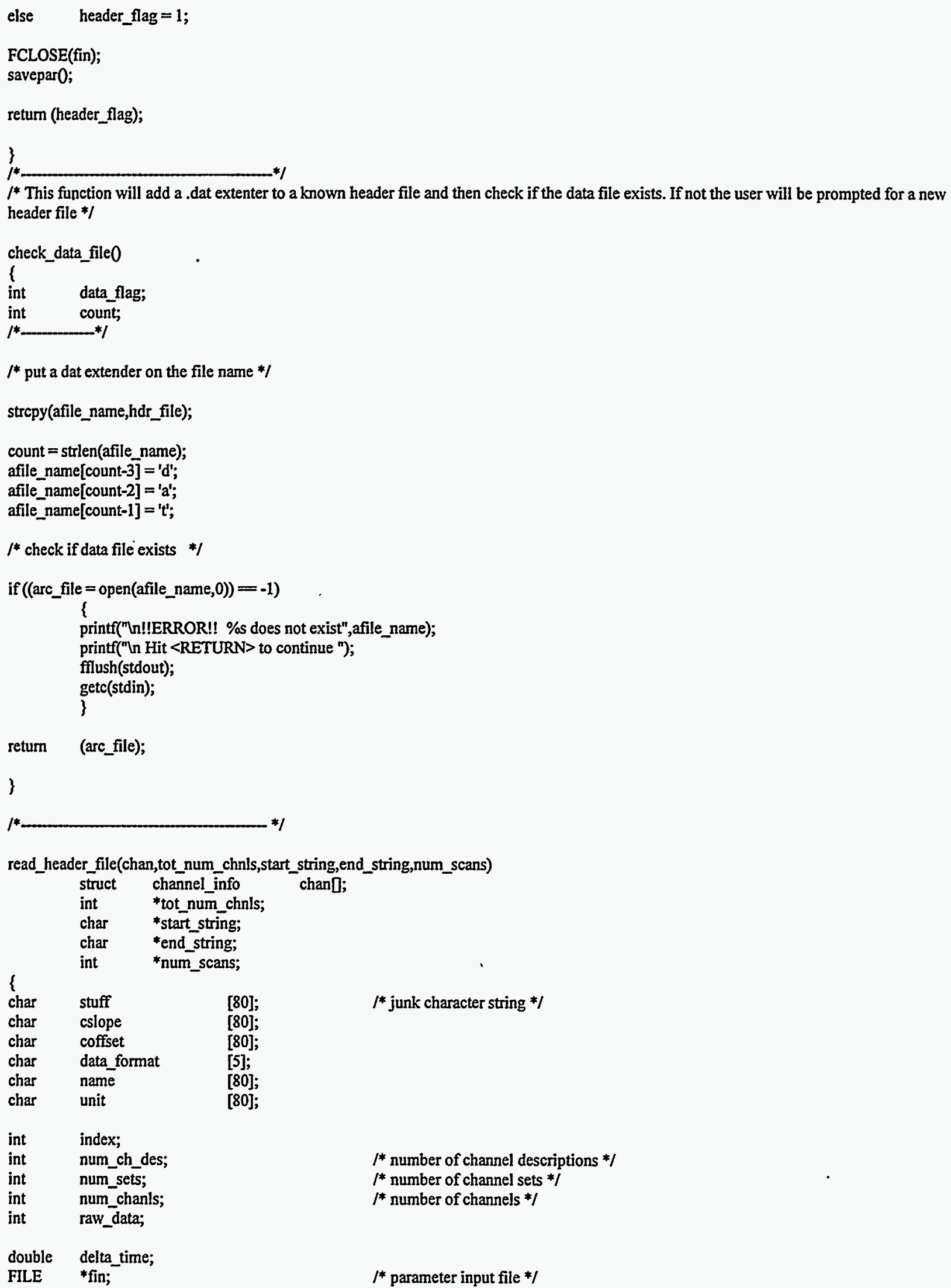




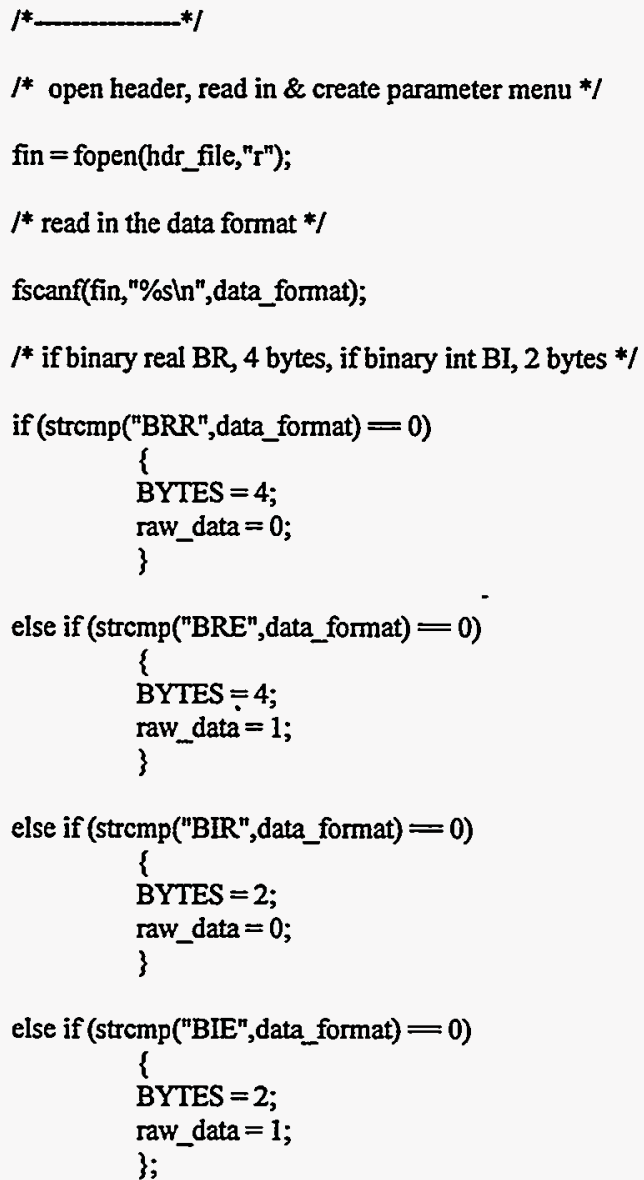

$f *$ read in daves comment string $* /$

strcpy(stuff,read_string(fin));

$I^{*}$ read in the file start and end times *I

strcpy(start_string,read_string(fin)); strcpy(end_string,read_string(fin));

$I^{*}$ read in the number of scans *I

fscanf(fin, "\%d \%lfln",num_scans,\&delta_time);

$/ *$ read in the number of channel descriptions \& the descriptions *I

fscanf(fin, "\%dln",\&num_ch_des);

for $\left(i=1 ; i<=n u m \_c h \_d e s ;+; i\right)$

strcpy(stuff,read_string(fin));

$/ *_{\text {read }}$ in the number of sets */

fscanf(fin, "\%dln",\&num_sets);

index $=0 ; \quad \quad \quad / *$ index for channel names */

for(i=0;i<num_sets;++i)

\{

/* read in the set number, description, version, \& date taken */

for $(k=1 ; k<=4 ; \div+4)$ 
strcpy(stuff,read_string(fin));

$I^{*}$ read in the number of channels */

fscanf(fin,"\%din",\&num_chanls);

for $(j=0 ; j<$ num_chanls; $++j)$

\{

$I^{*}$ calculate the random access data offset location *I

chan $[$ index $+\mathrm{j}] . l o c=$ BYTES * $($ index $+j)$;

I* skip 3 lines *I

for $(k=1 ; k<=3 ;++k)$

strcpy(stuff,read_string(fin));

I* read in the channel offset and slope as strings *I

strcpy(cslope,read_string(fin));

strcpy(coffset,read_string(fin));

$I^{*}$ read the slope and offset from the above strings *I

$l^{*}$ if error reading then set slope 1 , and offset $0 * I$

if (sscanf(cslope, "\%lf",\&chan [index+j].slope) != 1)

chan $[$ index+j].slope $=1.0$;

if (sscanf(coffset, "\%lf", \&chan[index+j].offset) != 1) chan [index+j].offset $=0.0$;

/* skip 2 lines */

for $(k=1 ; k<=2 ;++k)$

strcpy(stuff,read_string(fin));

$1 *$ read in the channel max \& min values *I

strcpy(stuff,read_string(fin));

sscanf(stuff," \%f",\&chan[index+j].max);

strcpy(stuff,read_string(fin));

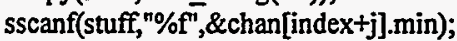

I* skip 1 line *I

strcpy(stuff,read_string(fin));

/* read in the channel name, \& units */

strcpy(chan[index+j].name,read_string(fin));

strcpy(chan[index+j].unit,read_string(fin));

I* skip to the end of the channel des *I

for $(k=13 ; k<=$ num_ch_des; $++k)$

\}

strcpy(stuff,read_string(fin));

index $=$ index $+j$

*tot_num_chnls = index;

$I^{*}$ set the record length

REC_LEN $=$ *tot num_chnls * BYTES;

if (REC_LEN $!=\bar{R} E C \_\bar{I}$ _SIZE)

\{ 
printf ("InTHIS IS NOT A \%d-BYTE RECORD FILE!!n",REC_IN_SIZE); printf ("REC_LEN = \%dln", REC_LEN);

exit (-1);

\}

I* close the header file

*1

FCLOSE(fin);

\}/* read_header_file *I 


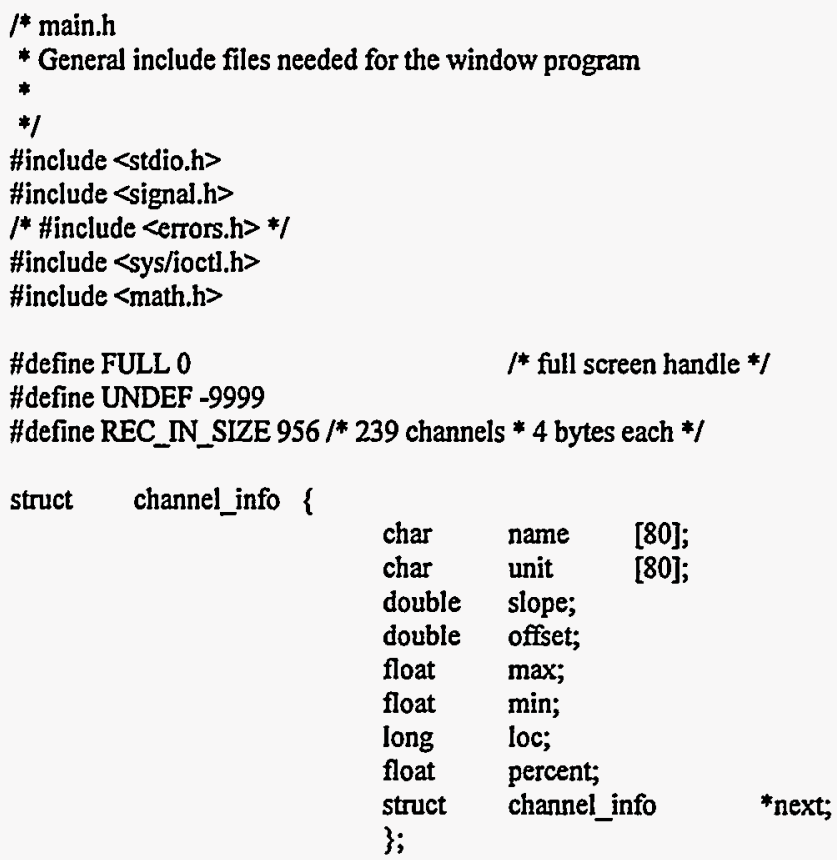

long REC_LEN;

The following two files are also necessary to properly run CYCCAT.C:

1. makefile:

\# MAKEFILE for cycle count software

CFLAGS $=-g$

FILES $=$ cyccat.o read.o ben_io.c

MODULES $=\$$ (FILES $)$

\# compile \& link files

cycle: \$(MODULES)

ce \$(CFLAGS) \$(MODULES) -0 cyccat -lm

S(MODULES) : main.h

2. last_bin.dat: 
Appendix C

\section{BESTCYC.F}


program bestcyc

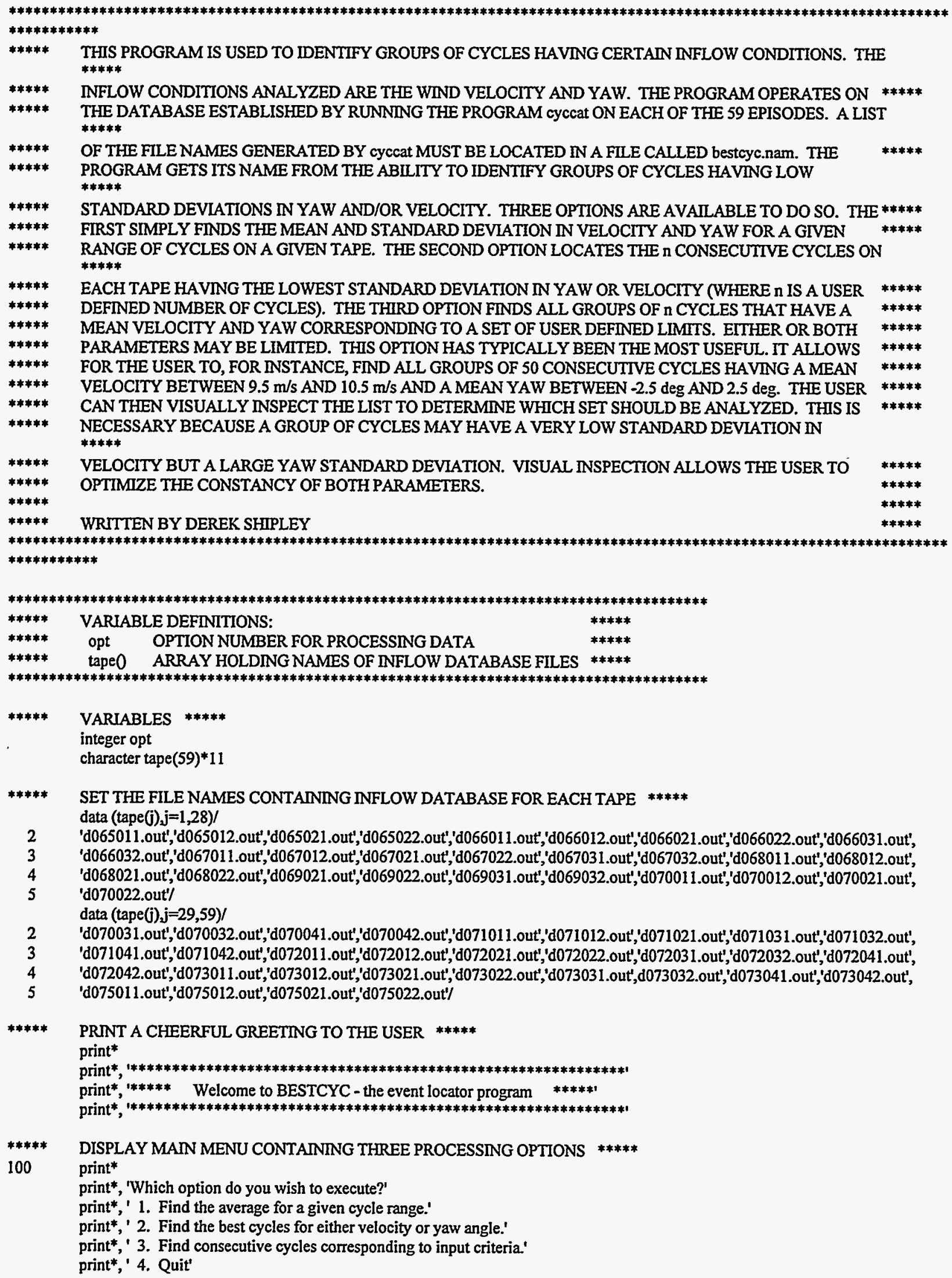

***** DISPLAY MAN MENU CONTAINING THREE PROCESSING OPTIONS *****

100 print*

print*, 'Which option do you wish to execute?'

print*,' 1 . Find the average for a given cycle range.'

print*, ' 2. Find the best cycles for either velocity or yaw angle.'

print*,' 3 . Find consecutive cycles corresponding to input criteria.'

print*', 4. Quit' 
write $(6,1)$

1 format('Enter the desired option number (1-4): '\$)

read*, opt

***** BRANCH TO PROPER OPTION *****

if (opteq.1) then

call avegroup(tape)

elseif (opt.eq.2) then

call bestset(tape)

elseif (opt.eq.3) then

call fitcrit(tape)

elseif (opt .eq. 4) then

goto 200

else

print*,'Invalid option number. Please try again.'

goto 100

endif

goto 100

continue

stop

end

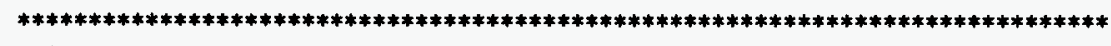

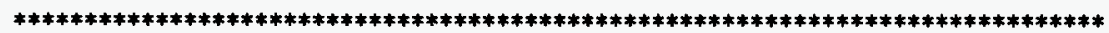

subroutine avegroup(tape)

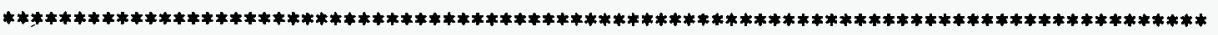

***** OPTION 1 - DETERMINE THE AVERAGE YAW AND VELOCITY AND

***** THEIR STANDARD DEVIATIONS FOR A GIVEN GROUP OF CYCLES *****

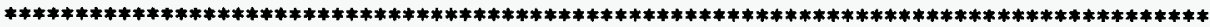

\begin{tabular}{|c|c|c|c|}
\hline$* * * * *$ & \multicolumn{2}{|c|}{ VARIABLE AND PARAMETER DEFINITIONS: } & \multirow{2}{*}{$\begin{array}{l}* * * * * \\
* * * * *\end{array}$} \\
\hline ***** & cyco & CYCLENUMBER OF EACH DATABASE ENTRY & \\
\hline ***** & cychigh & UPPER BOUNDARY OF PRESENT CYCLE GROUPING & $* * * * *$ \\
\hline 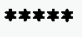 & cyclow & LOWER BOUNDARY OF PRESENT CYCLE GROUPING & ****** \\
\hline ***** & data() & DATA READ FROM INFLOW DATABASE FILES & ***** \\
\hline ***** & infil & INPUT FILE NAME & $* * * * *$ \\
\hline ***** & MAXCYC & MAXIMUM NUMBER OF CYCLES PER TAPE & ****** \\
\hline$* * * * *$ & NCOL & NUMBER OF DATA COLUMNS IN DATABASE FILES & $* * * * *$ \\
\hline ***** & NUMTAPE & NUMBER OF DATA TAPES & $* * * * *$ \\
\hline ***** & tape 0 & ARRAY HOLDNG NAMES OF NFLOW DATABASE FILES & ****** \\
\hline ****** & vel0 & INFLOW VELOCITY OF EACH DATABASE ENTRY & ***** \\
\hline ***** & velsig 0 & VELOCITY STANDARD DEVIATION OF EACH ENTRY & ***** \\
\hline ***** & velmean & MEAN VELOCITY OF PRESENT CYCLE GROUPING & $* * * * *$ \\
\hline ****** & velsd & VELOCITY STANDARD DEVIATION OF PRESENT CYCLES & $* * * * *$ \\
\hline ***** & velsdsum & SUM OF VELOCITY RESIDUALS SQUARED & $* * * * *$ \\
\hline ***** & velsum & SUM OF VELOCITIES IN PRESENT CYCLE GROUPING & ***** \\
\hline ****** & yaw0 & YAW OF EACH DATABASE ENTRY & $* * * * *$ \\
\hline ****** & yawsig0 & YAW STANDARD DEVIATION OF EACH DATABASE ENTRY & $* * * * *$ \\
\hline ***** & yawmean & MEAN YAW OF PRESENT CYCLE GROUPING & $* * * * *$ \\
\hline ***** & yawsd & YAW STANDARD DEVIATION OF PRESENT CYCLES & $* * * * *$ \\
\hline$* * * * *$ & yawsdsum & SUM OF YAW RESIDUALS SQUARED IN & ****** \\
\hline$* * * * *$ & yawsum & SUM OF YAW IN PRESENT CYCLE GROUPNG & ***** \\
\hline \multicolumn{4}{|c|}{$\neq * * * * * * * * * * * * * * * * * * * * * * * * * * * * * * * * * * * * * * * * * * * * * * * * * * * * * * * * * * * * * * * * * * * * * * * * * * * * * * * * * * * * * * * * * * * * * * * * * * * * * * * * * *$} \\
\hline$* * * * *$ & \multicolumn{3}{|c|}{$\begin{array}{l}\text { DECLARE VARIABLES AND CONSTANTS ***** } \\
\text { parameter (MAXCYC=450) } \\
\text { parameter (NCOL=9) } \\
\text { parameter (NUMTAPE=59) } \\
\text { real data(NCOL,MAXCYC),vel(MAXCYC),yaw(MAXCYC),cyc(MAXCYC) } \\
\text { real velsig(MAXCYC),yawsig(MAXCYC) } \\
\text { real yawsum,yawmean,yawsdsum,yawsd } \\
\text { real velsum,velmean,velsdsum,velsd } \\
\text { integer cyclow,cychigh } \\
\text { character infil*11,tape(NUMTAPE)*11 }\end{array}$} \\
\hline
\end{tabular}


***** PROMPT THE USER FOR THE INPUT FIL E AND OPEN IT *****

100

print*

write(6,1)

1 format('Enter the name of the input file (ext = .out): '\$) read*, infil

open (unit=11, file=infil,iostat=inerr,status='old)

if (inerr .ne. o) then

print*, 'File does not exist. Please try again.'

goto 100

endif

***** PROMPT THE USER FOR THE LOWEST CYCLE TO BE AVERAGED

print*

- write $(6,2)$

2 format('Enter the lowest cycle to average: '\$)

read $^{*}$, cyclow

***** PROMPT THE USER FOR THE HIGHEST CYCLE TO BE AVERAGED ***** write $(6,3)$

3 format('Enter the highest cycle to average: '\$) read*, cychigh

***** READ IN ALL THE DATA FROM THE GIVEN FILE *****

$\mathrm{n}=2$

$15 \operatorname{read}(11, *$,end $=16)($ data $(j, n), j=1, N C O L)$

$\operatorname{cyc}(n)=\operatorname{data}(1, n)$

$\operatorname{vel}(n)=\operatorname{data}(2, n)$

velsig $(\mathrm{n})=\operatorname{data}(3, \mathrm{n})$

yaw $(n)=\operatorname{data}(4, n)$

yawsig $(n)=\operatorname{data}(5, n)$

$n=n+1$

goto 15

16 continue

close (11)

***** CALCULATE THE MEAN YAW AND VELOCITY FOR THE SPECIFIED CYCLES

velsum $=0$

yawsum $=0$

do $18, \mathrm{i}=$ cyclow, cychigh

velsum $=$ velsum+vel(i)

yawsum $=$ yawsum+yaw $(\mathbf{i})$

18 continue

velmean $=$ velsum $/($ cychigh-cyclow +1$)$

yawmean $=$ yawsum $/($ cychigh-cyclow +1$)$

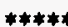

CALCULATE THE VELOCITY AND YAW STANDARD DEVIATIONS

velsdsum $=0$

yawsdsum $=0$

do $19, \mathrm{j}=$ cyclow, cychigh

velsdsum $=$ velsdsum+velsig(j)**2+(vel(j)-velmean $)^{* * 2}$

$19 \quad$ yawsdsum $=$ yawsdsum + yawsig $(j) * * 2+(\text { yaw }(j) \text {-yawmean })^{* * 2}$

continue

velsd $=$ sqrt $($ velsdsum $/($ cychigh-cyclow +1$))$

yawsd $=$ sqrt (yawsdsum/(cychigh-cyclow+1))

***** PRNT THE RESULTS TO THE SCREEN *****

print*

print*, 'The results for cycles',cyclow,' -',cychigh,' is:'

print 9000

print9100,infil,cyclow,cychigh,yawmean,yawsd,velmean,velsd

print*

print* 


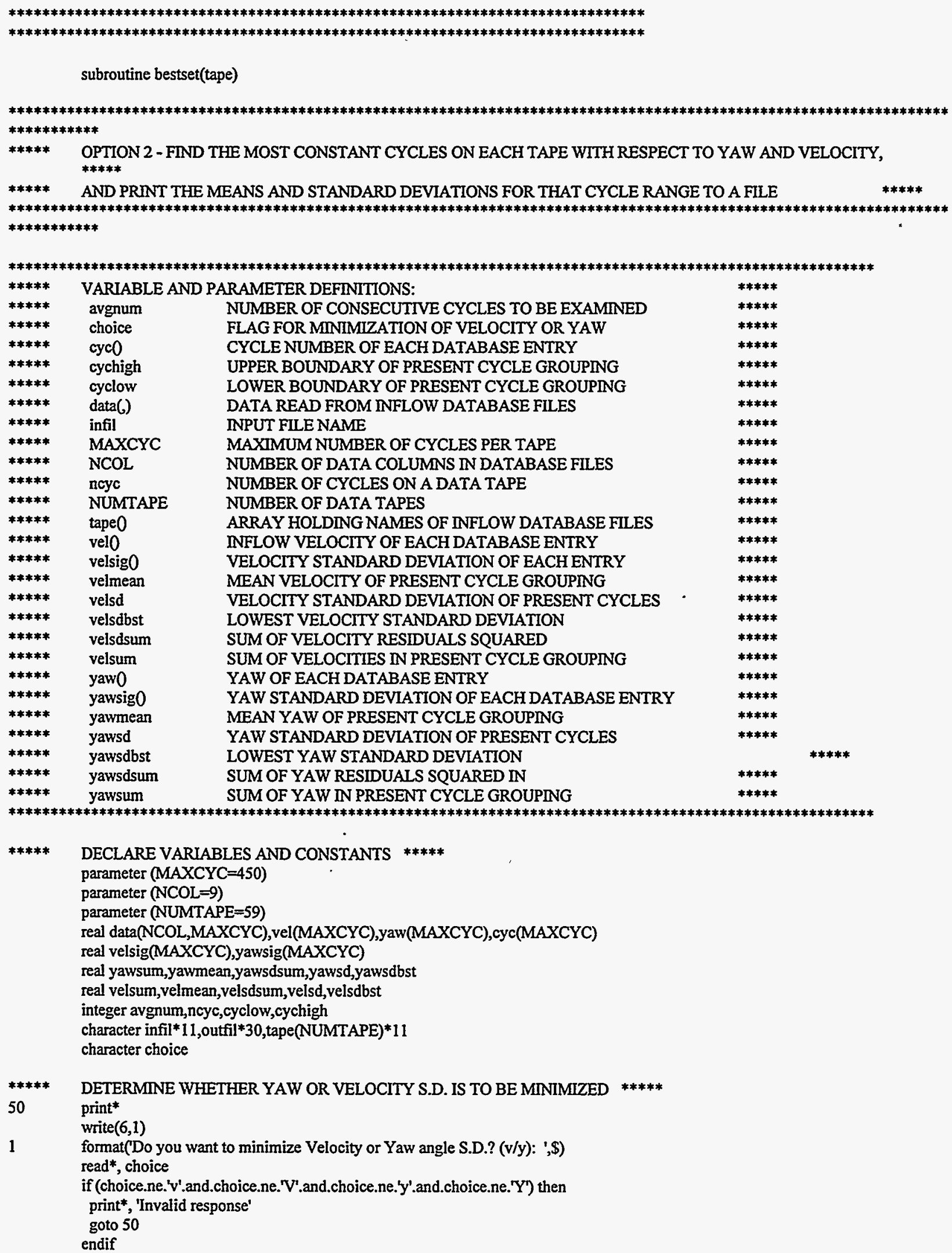


print*

write $(6,2)$

2 format('Enter the number of consecutive cycles to evaluate: '\$)

read*, avgnum

PROMPT THE USER FOR THE NAME OF THE OUTPUT FILE AND OPEN IT *****

print*

3 format('Enter the name of the output file: ',\$)

read*, outfil

open (unit=16, file=outfil, status='unknown')

WRITE A HEADER TO THE TOP OF THE OUTPUT FILE

if (choice.eq.'y'.or.choice.eq.' 'Y') then

write $\left(16,{ }^{*}\right), \quad$ The most constant',avgnum,' cycles in terms of the yaw angle for each tape:'

write $(16,9000)$

else

write $\left(16,{ }^{*}\right), \quad$ The most constant',avgnum,' cycles in terms of velocity for each tape:'

write $(16,9000)$

endif

***** OPEN EACH INFLOW DATABASE FILE *****

\section{do $700, n=1$, NUMTAPE}

infil $=$ tape $(n)$

$\mathrm{k}=2$

open (unit=11, file=infil,status='old')

***** READ IN ALL DATA FROM EACH TAPE AND PLACE IT IN ARRAYS *****

$100 \quad \operatorname{read}(11, *$,end $=200)($ data $(j, k), j=1, N C O L)$

$\operatorname{cyc}(\mathrm{k})=\operatorname{data}(1, \mathrm{k})$

$\operatorname{vel}(k)=\operatorname{data}(2, k)$

velsig $(k)=\operatorname{data}(3, k)$

yaw $(k)=\operatorname{data}(4, k)$

yawsig $(k)=\operatorname{data}(5, k)$

$\mathrm{k}=\mathrm{k}+1$

goto 100

200

continue

close (11)

ncyc $=k-1$

print*,n,ncyc

CALCULATE THE MEAN VELOCITYU AND YAW FOR EACH POSSIBLE BLOCK OF avgnum CYCLES

velsdbst $=200$

yawsdbst $=200$

do $300, \mathrm{i}=2$, ncyc-avgnum

velsum $=0$

yawsum $=0$

do $400, \mathrm{j}=1$,avgnum

velsum $=$ velsum+vel $(i+j-1)$

400

yawsum $=$ yawsum+yaw $(i+j-1)$

continue

yawmean $=$ yawsum/avgnum

velmean $=$ velsum/avgnum

$\star \star * * *$

CALCULATE THE VELOCITY AND YAW S.D. FOR EACH BLOCK *****

velsdsum $=0$

yawsdsum $=0$

do $500, \mathrm{j}=1$, avgnum

velsdsum=velsdsum+velsig( $i+j-1) * * 2+($ vel $(i+j-1)$-velmean $) * * 2$

500 yawsdsum=yawsdsum+yawsig $(i+j-1) * * 2+($ yaw $(i+j-1)$-yawmean $) * * 2$ continue

velsd=sqrt(velsdsum/(avgnum-1))

yawsd=sqrt(yawsdsum/(avgnum-1))

**** DETERMINE WHETHER THIS COMBINATION IS THE MOST CONSTANT *****

if (choice.eq.'y'.or.choice.eq.' 'Y') then 


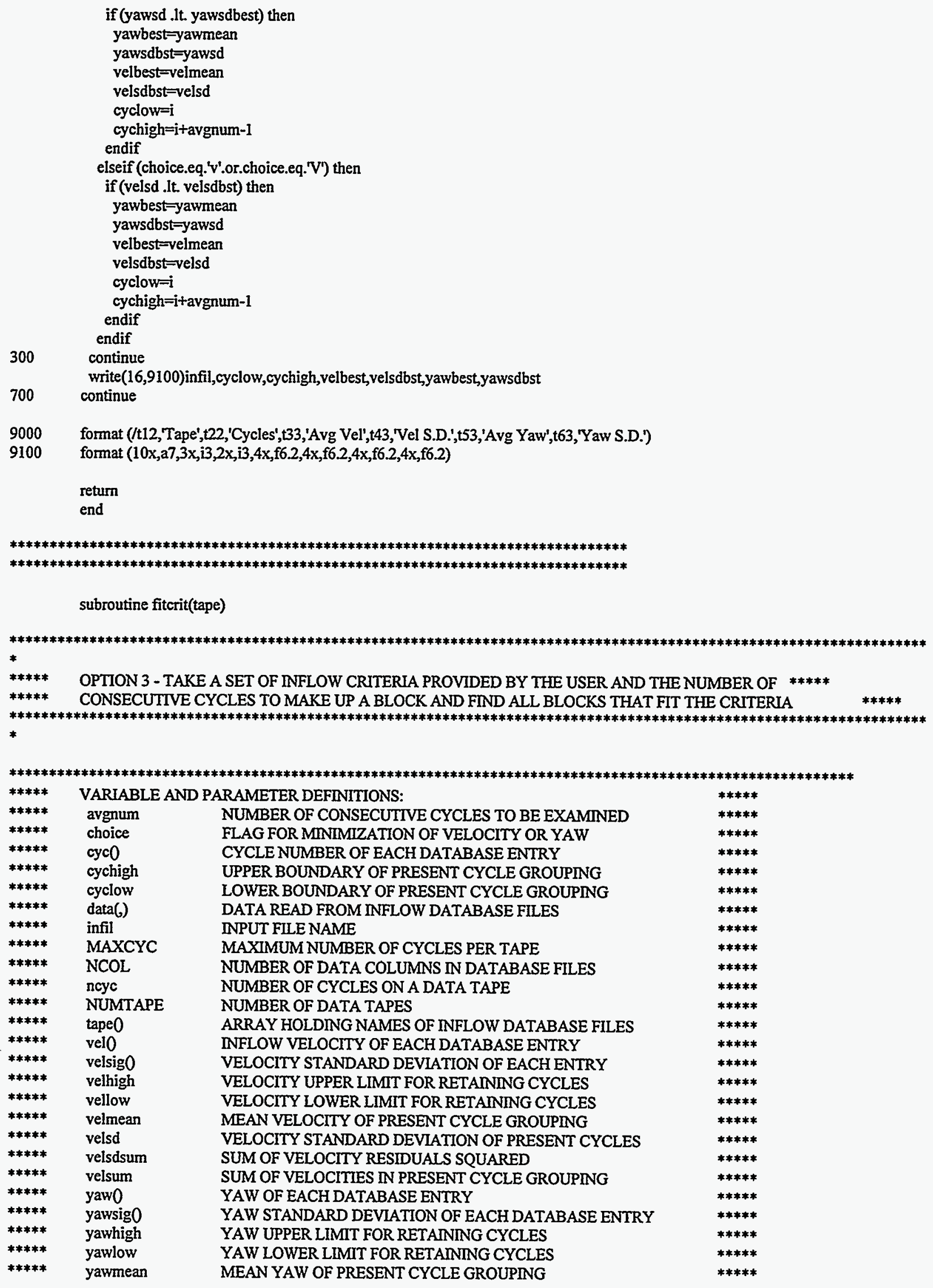




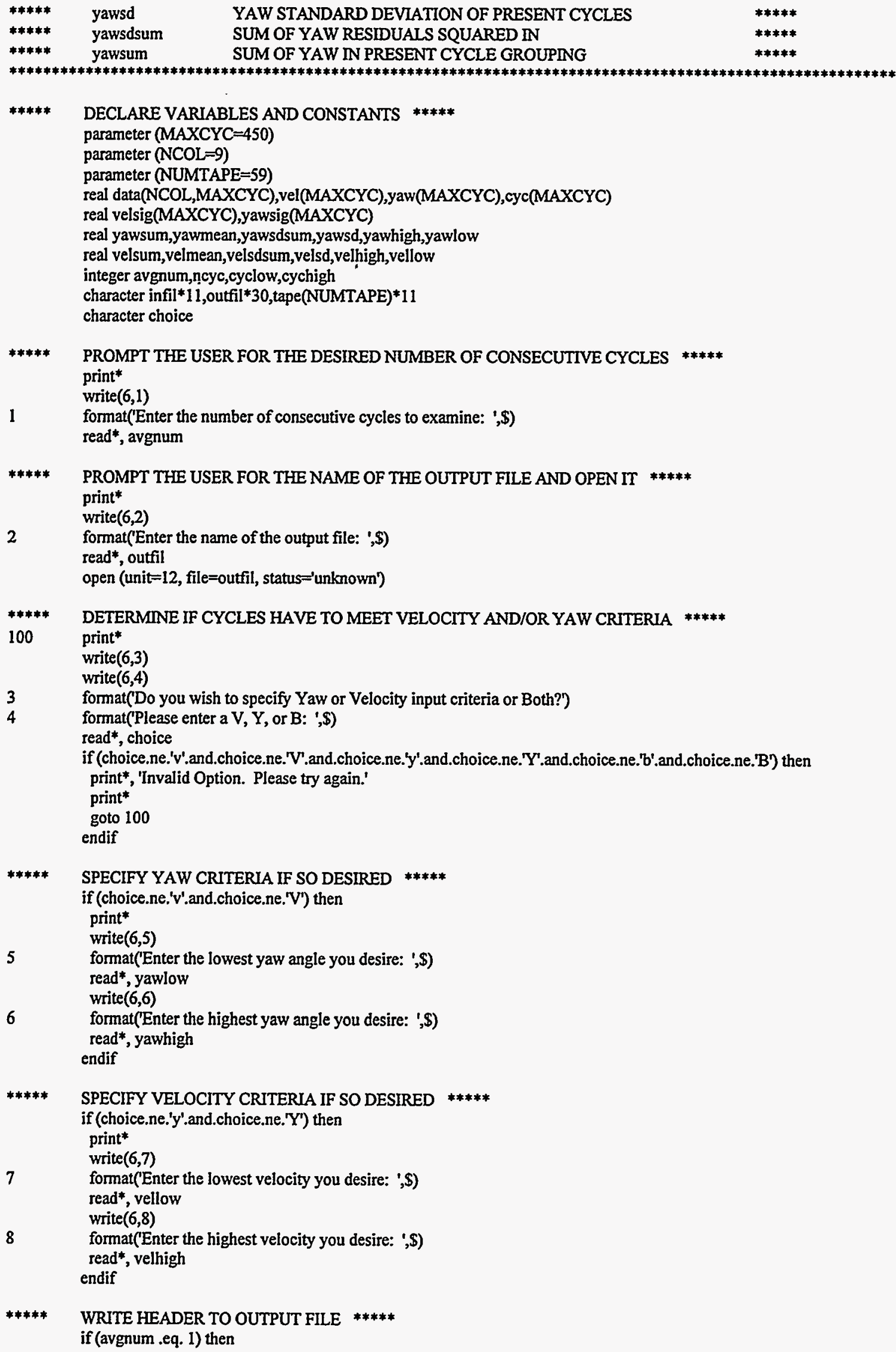

***** SPECIFY YAW CRITERIA IF SO DESIRED *****

if (choice.ne.' $v$ '.and.choice.ne.' $V$ ') then print* 
write $\left(12,{ }^{*}\right)$, 'All cycles that meet the following criteria:"

else

write $(12, *)$, 'All combinations of, avgnum,' consecutive cycles that meet the following critera:'

endif

write $(12, *)$

if (choice.ne.'y'.and.choice.ne.' 'Y') write(12,9200), vellow,velhigh

if (choice.ne.'v'.and.choice.ne.' 'V') write(12,9300),yawlow,yawhigh

if (avgnum .eq. 1) then

write(12,9050)

else

write $(12,9000)$

endif

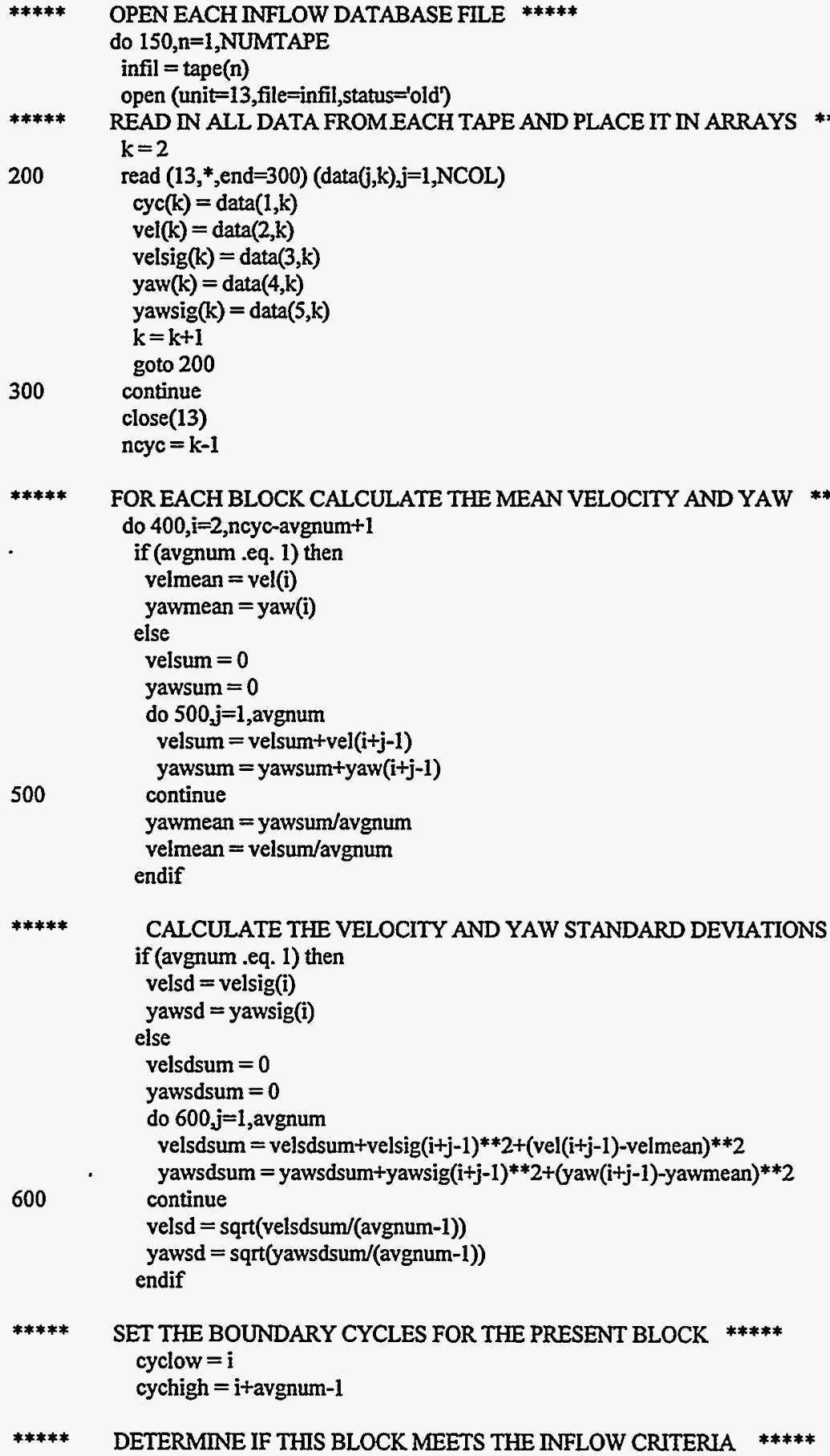


$\# * * * *$

650

400

IF IT DOES, THEN WRITE THE DATA TO THE OUTPUT FILE

if (choice .eq. ' $y$ ' .or. choice .eq. 'Y') then

if (yawmean .le. yawhigh and. yawmean .ge. yawlow) then

if (avgnum .eq. 1) then

write $(12,9150)$ infil,cyclow,velmean,velsd,yawmean,yawsd

else

write $(12,9100)$ infil,cyclow,cychigh,velmean,velsd,yawmean,yawsd

endif endif

elseif (choice.eq.'v'.or.choice.eq.' $\mathrm{V}$ ') then

if (velmean .le. velhigh .and. velmean .ge. vellow) then

if (avgnum .eq. 1) then

write $(12,9150)$ infil,cyclow,velmean,velsd,yawmean,yawsd

else

write $(12,9100)$ infil,cyclow,cychigh,velmean,velsd,yawmean,yawsd

endif

endif

else

if (yawmean .le. yawhigh .and. yawmean .ge. yawlow .and.velmean .le. velhigh .and. velmean .ge. vellow) then if (avgnum .eq. 1) then

write $(12,9150)$ infil,cyclow,velmean,velsd,yawmean,yawsd

else

write $(12,9100)$ infil,cyclow,cychigh,velmean,velsd,yawmean,yawsd

endif

endif

endif

continue

continue

format (/t12,'Tape',t22,'Cycles',t33,'Avg Vel',t43,'Vel S.D.',t53,'Avg Yaw',t63,'Yaw S.D.')

format (/t12,'Tape',t22,'Cycle',t32,'Avg Vel',t42,'Vel S.D.',t52,'Avg Yaw',t62,'Yaw S.D.')

format (10x,a7,3x,i3,2x,i3,4x,f6.2,4x,f6.2,4x,f6.2,4x,f6.2)

format $(10 \mathrm{x}, \mathrm{a} 7,5 \mathrm{x}, \mathrm{i} 3,6 \mathrm{x}, \mathrm{f} 6.2,4 \mathrm{x}, \mathrm{f6} .2,4 \mathrm{x}, \mathrm{f6} .2,4 \mathrm{x}, \mathrm{f} 6.2)$

format ('Average velocity between ',f5.2,' and ',f5.2,' $\mathrm{m} / \mathrm{s}$ ')

format ('Average yaw angle between ',f6.2,' and ',f6.2,' degrees')

return

end 
Appendix D

\section{AVCNCT.C \\ DIRECTORY.DAT \\ MAKEFILE \\ LAST_BIN.DAT}


This program reads 400-byte, 101 -datum records containing 1 pressure profile.

This version reads BRE format files - binary 4-byte reals, engineering units. For each cycle of data it computes the mean and standard deviation of specified channels, ct \& $\mathrm{cn}$ (one cycle consists of one set of data with azimuthal angle from 0 to 360 degrees). The data from specified channels written to 3 ASCII files, hdr_file.out, one for $\mathrm{cn} \&$ one for ct means \& std dev, and one for $\mathrm{cn} \& \mathrm{ct}$ maximums. This file may be used as-is to plot out data from each cycle. The data is also written to user files, hdr_file.sum. The user files contain headings.

The following channels are specified:

$\begin{array}{ll}\# 203 & \text { Normal Force Cn(80\%) } \\ \# 205 & \text { Normal Force Cn(63\%) } \\ \# 204 & \text { Normal Force Cn(47\%) } \\ \# 206 & \text { Normal Force Cn(30\%) } \\ & \\ \# 207 & \text { Normal Force Ct(80\%) } \\ \# 208 & \text { Normal Force Ct(63\%) } \\ \# 209 & \text { Normal Force Ct(47\%) } \\ \# 210 & \text { Normal Force Ct(30\%) }\end{array}$

NOTE: If using COMBINED EXPERIMENT PHASE II - REDUCED DATA. FILE SUMMARY for the channels (when modifying program), the correct mydata index number is the listed channel \# - 1 . For example, $\mathrm{Cn}(80 \%)$ is listed as channel $\# 204$, so 203 is used here.

This version calls cln.c $\rightarrow$ cln(float datarray, int numpts, int found) to remove anomalous points more than $\mathrm{X}$ standard deviations from the mean. (At this writing, cin removes points which change more than 2 (or 3$)^{*}$ standard deviations from the previous point.) If anomalous points are found, the cycle number is recorded in a hdr_file.err file.

INPUT: Raw binary data from Combined Experiment optical tapes

OUTPUT:

hdr_filecn.out \& hdr_filecn.sum cycle\# 30\%span_cnt-sd $47 \%$ span_cnt-sd $63 \%$ span_cnt-sd $80 \%$ span_cnt-sd

hdr_filect.out \& hdr_filect.sum cycle\# 30\%span_ct+-sd 47\%span_ct+-sd 63\%span_ctt-sd $80 \%$ span_ct+-sd

hdr_filemax.out \& hdr_filemax.sum cycle\# cn values for: $30 \%$ spanmax $47 \%$ spanmax $63 \%$ spanmax $80 \%$ spanmax hdr_file.err ct values for: $30 \%$ spanmax $47 \%$ spanmax $63 \%$ spanmax $80 \%$ spanmax

anomalous point found in cycle\#

WRITTEN:

M. Querijero, T. Young

SERI/WIND

\section{/* DECLARATION OF GLOBAL VARIABLES */}

\#define DEFALT AZ CHANL 57

\#define NCHMA $\bar{X}$

\#define NPTMAX

\#define NCYCMAX

\#define TRUE

\#define FALSE

\#define span

\#define cn30

\#define ct30

\#define cnmagnitude 2

\#define ctmagnitude 3

1

0
$I^{*}$ default azimuth angle channel $* I$

I* max number of channels *I

/* max number of pts/cycle */

I* max \# cycles, $72 \mathrm{rpm} * 5 \mathrm{~m} * I$

$$
440
$$

$4 \quad / *$ number of span locations of $\mathrm{Cn} \& \mathrm{Ct}$ data*/

$206 \quad / *$ mydata index number for $30 \%$ span on *I

$210 \quad 1 *$ mydata index number for $30 \%$ span ct *I

$/ *$ for cln 2* standard deviation for $\mathrm{cn} * /$

$l^{*}$ for $\operatorname{cln} 3^{*}$ stnadard deviation for ct *j

\#include "../old/main.h"

\#include <stdio.h>

\#include <fentl.h $>$

\#include <ermo.h>

\#include <string.h $>$

\#include <math.h>

\begin{tabular}{|c|c|c|c|}
\hline $\begin{array}{l}\text { extern } \\
\text { extern }\end{array}$ & $\begin{array}{l}\text { char } \\
\text { int } \\
\operatorname{cin} 0\end{array}$ & $\begin{array}{l}\text { *get_response0; } \\
\text { read_in_chanls0; }\end{array}$ & $\begin{array}{l}/ * \text { ben_io.c } * / \\
/ * \text { read.c } * /\end{array}$ \\
\hline
\end{tabular}




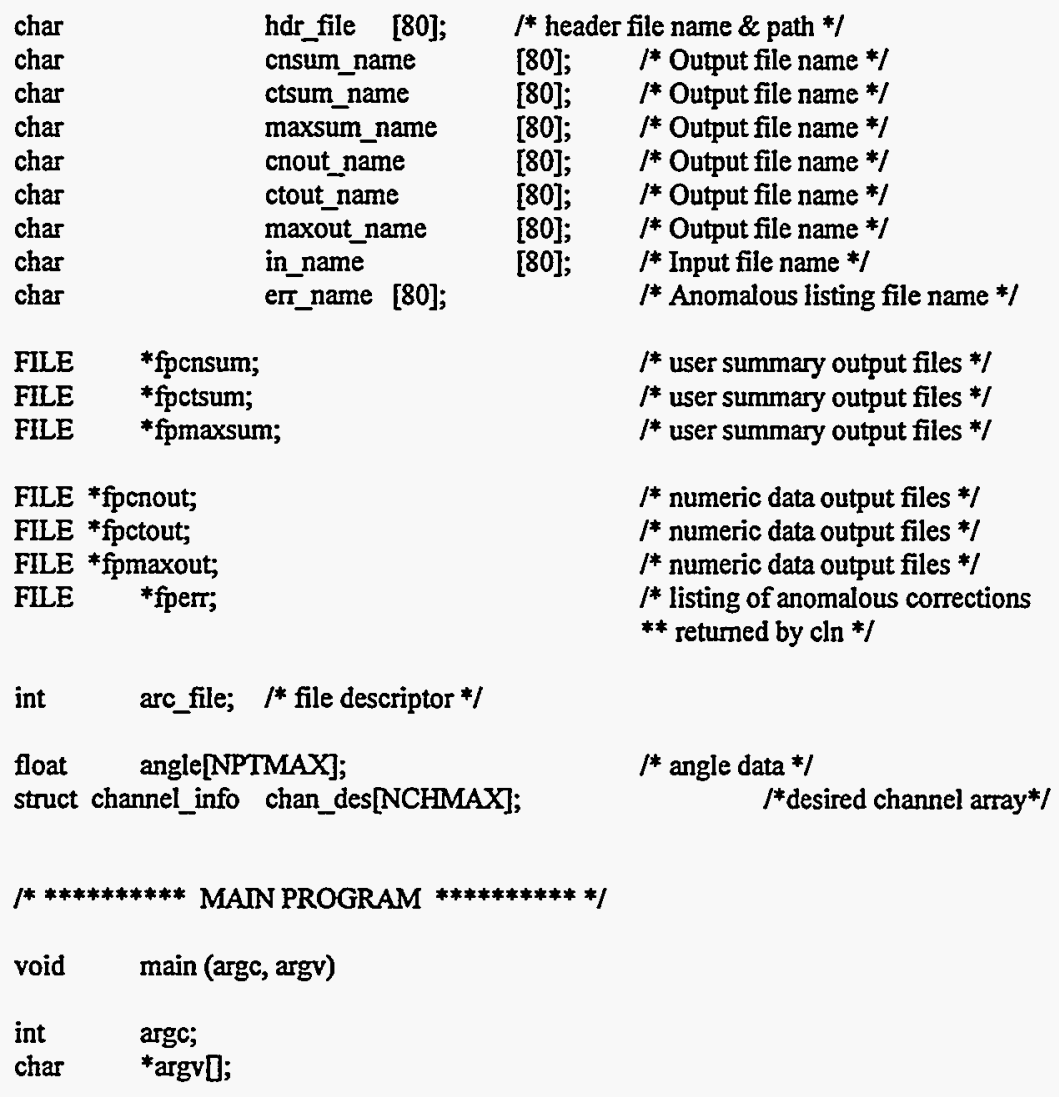

I* MAIN can be nun with or without arguments. argv and arge specify whether or not to read a file containing desired channels. ${ }^{*}$

f $\quad l^{*}$ begin main $* l$

/* VARIABLE DECLARATION - MAIN */

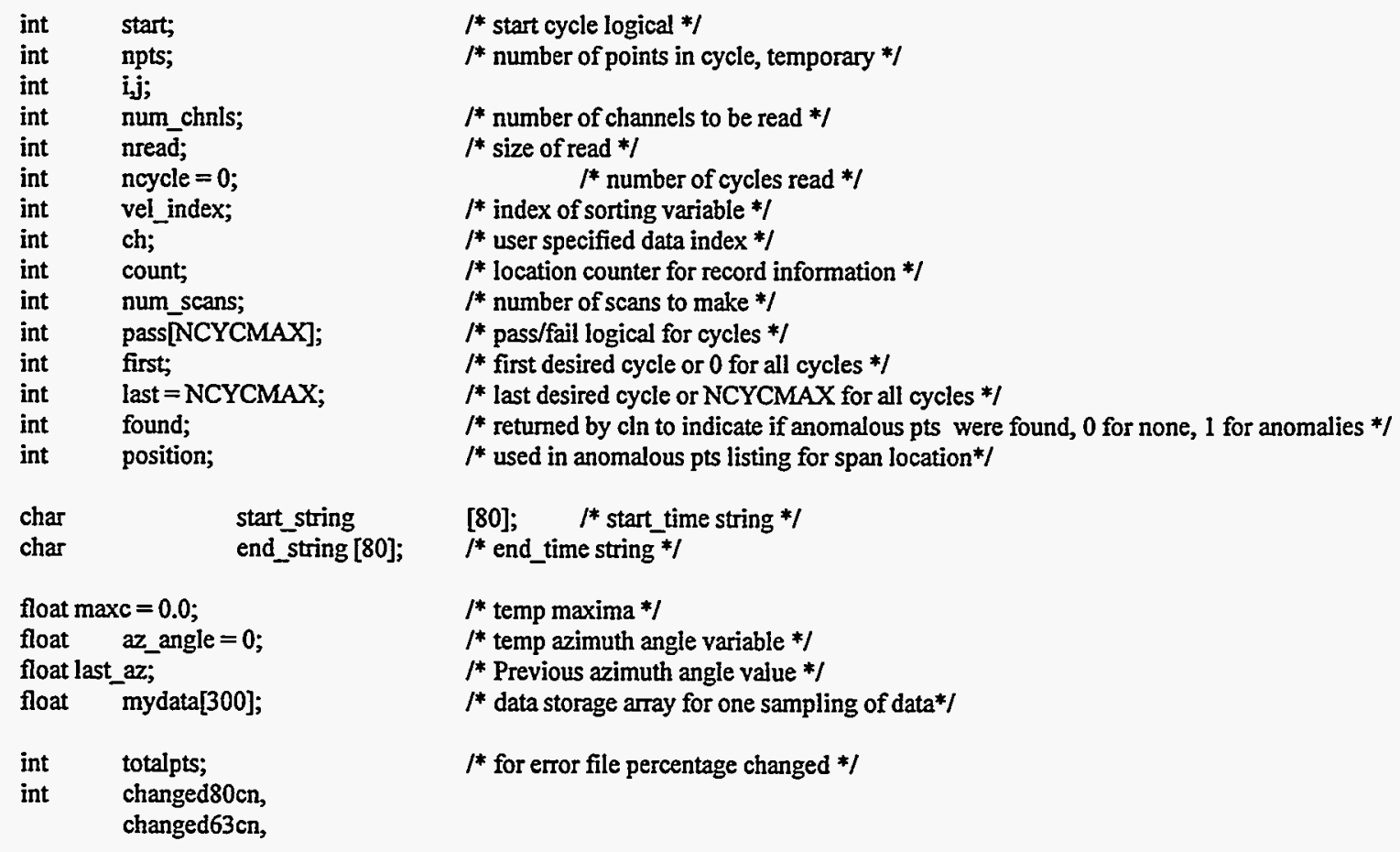




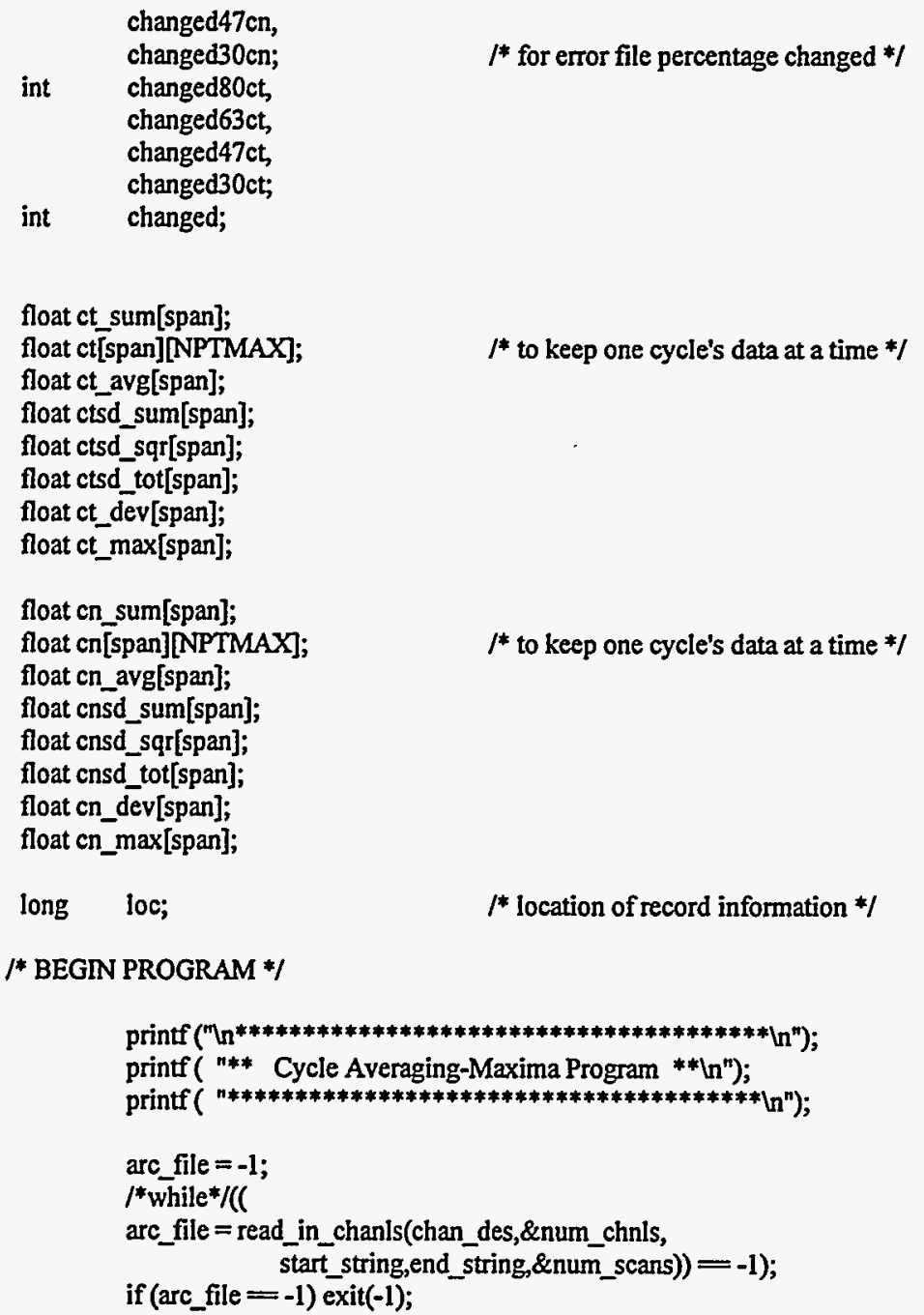

float ct_sum[span];

float ct[span][NPTMAX]; $\quad \quad I^{*}$ to keep one cycle's data at a time */

float ct_avg[span];

float ctsd sum[span];

float ctsd_sqr[span];

float ctsd tot[span]

float ct_dev[span];

float ct_max[span];

float cn_sum[span];

float cn[span][NPTMAX]; $\quad f^{*}$ to keep one cycle's data at a time */

float cn_avg[span];

float cnsd_sum[span];

float cnsd_sqrispan];

float cnsd tot[span];

float cn_dev[span];

float cn_max[span];

long loc;

/* location of record information *I

\section{j* BEGIN PROGRAM *}

$l^{*}$ get desired cycle numbers *I

(void)printf("You may select cycles or have all cycles included.ln"); (void)printf("Please enter the first desired cycle [0 for all]: "); (void)scanf("\%d",\&first);

if (first $>0$ )

\{

(void)printf("Please enter the last cycle: "); \} (void)scanf("\%d", \&last)

$f^{*}$ open up summary file *

if (strrchr(hdr_file, '/) != NULL) strcpy(in_name,strrchr(hdr_file, $/$ '/)+1);

else strcpy(in name, hdr file);

$/ *$ remove filename extension */

in_name[strlen(in_name)-4] $=10^{\prime}$;

/* output filenames *I

strcpy (cnsum name, in name);

strcpy (cnout_name, in_name);

strcpy (ctsum name, cnsum name)

$I^{*}$ these are user summary files * $/$

strcat (ctsum_name, "ct.sum");

strcpy (maxsum_name, cnsum_name); 


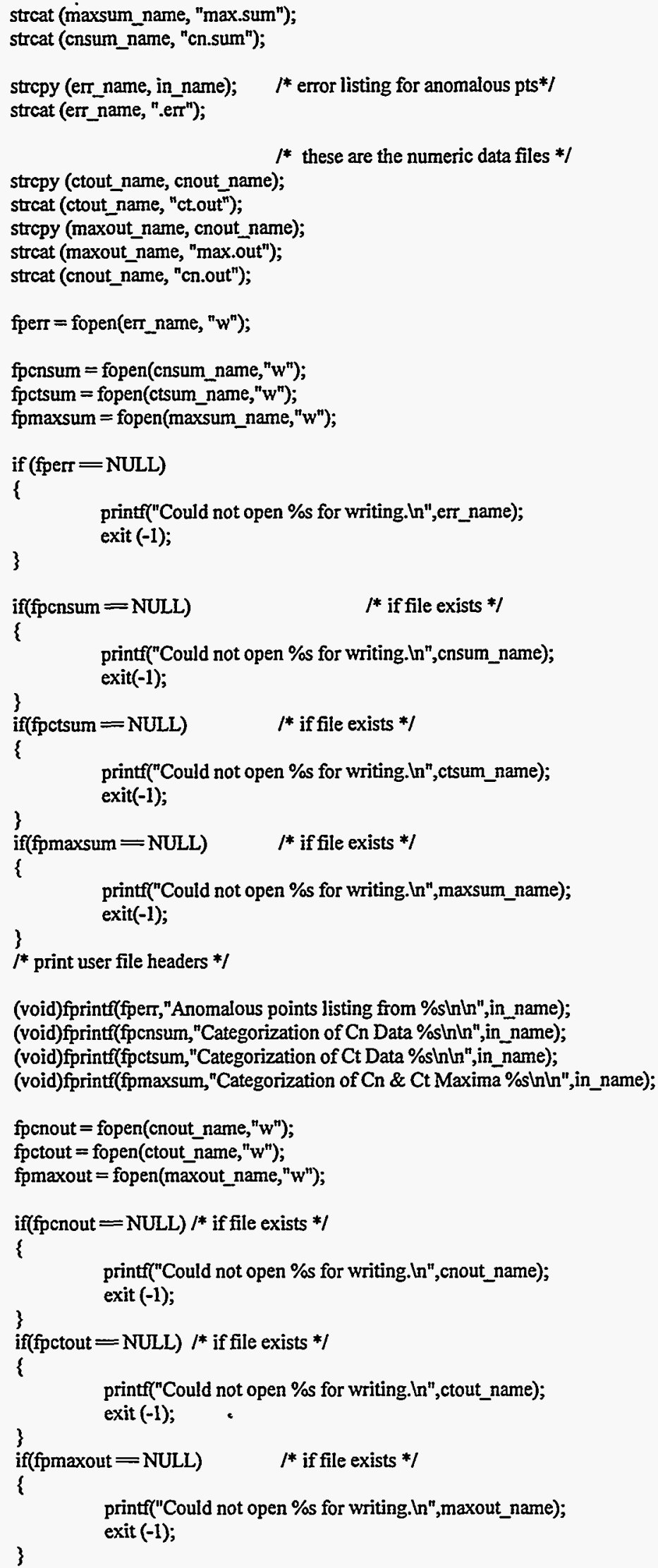


printf("InReading data file ... $\ln$ ");

$I^{*}$ set up first read */

count $=0 ; / *$ count indicates what record in file to get $* /$

nread $=$ REC_IN_SLZE; $\quad l^{*}$ bytes of data read */

loc $=$ count $*$ REC IN_SIZE;

Iseek (arc_file,loc, $\overline{0}$ );

start = FALSE; $\quad I^{*}$ logical for cycle indicator *

ncycle $=0$;

changed $=0$;

totalpts $=0$;

changed $80 \mathrm{cn}=$ changed $63 \mathrm{cn}=$ changed $47 \mathrm{cn}=$ changed $30 \mathrm{cn}=0$;

changed $80 \mathrm{ct}=$ changed $63 \mathrm{ct}=$ changed $47 \mathrm{ct}=$ changed $30 \mathrm{ct}=0$;

$I^{*}$ do the following for all records in the file, binary read of data *I

$I^{*}$ reads each channel of data from one time instance into mydata $\$$ */

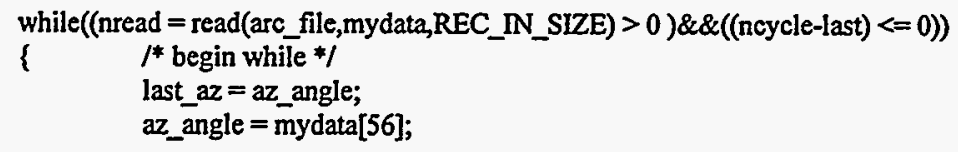

$I^{*}$ this series of if statements tests azimuthal angle to see what cycle it is in, whether to start or finish a cycle, etc. $* l$

$I^{*}$ check to see if we should start the cycle count *I

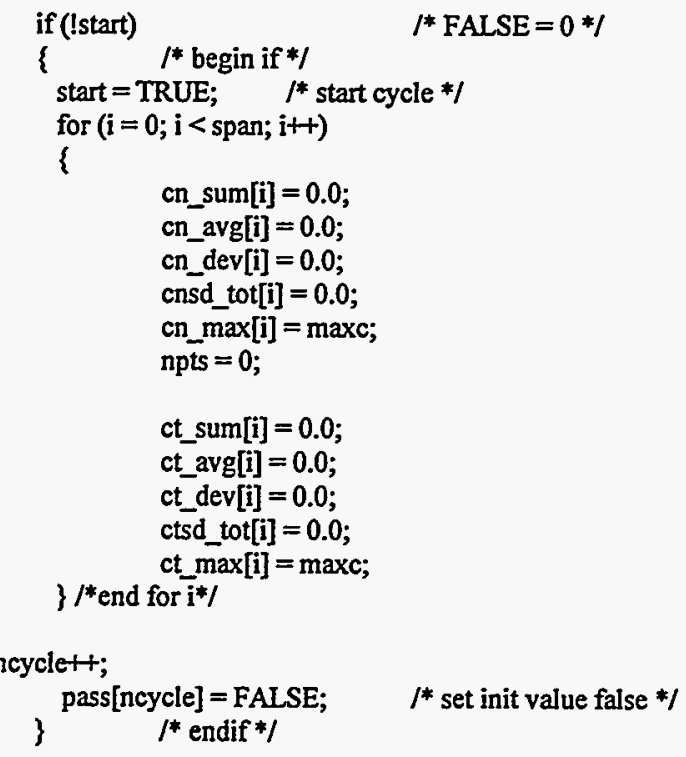

$I^{*}$ check to see if we should end the cycle count

** end the cycle if the azimuth angle has passed 360 degrees

** or if there are too many data points in the cycle, indicating

** an error in the azimuth angle

*I

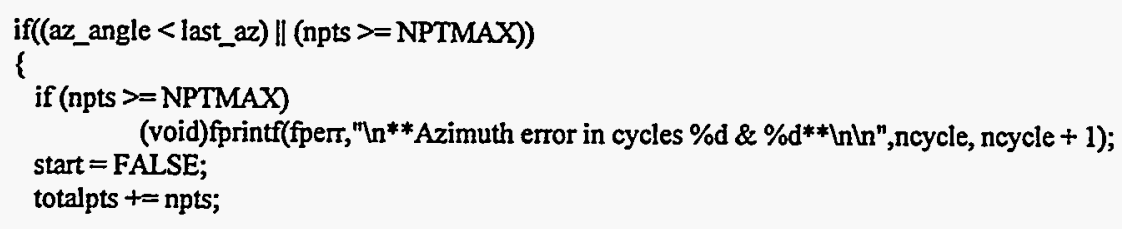

$l^{*}$ for each span location */ 


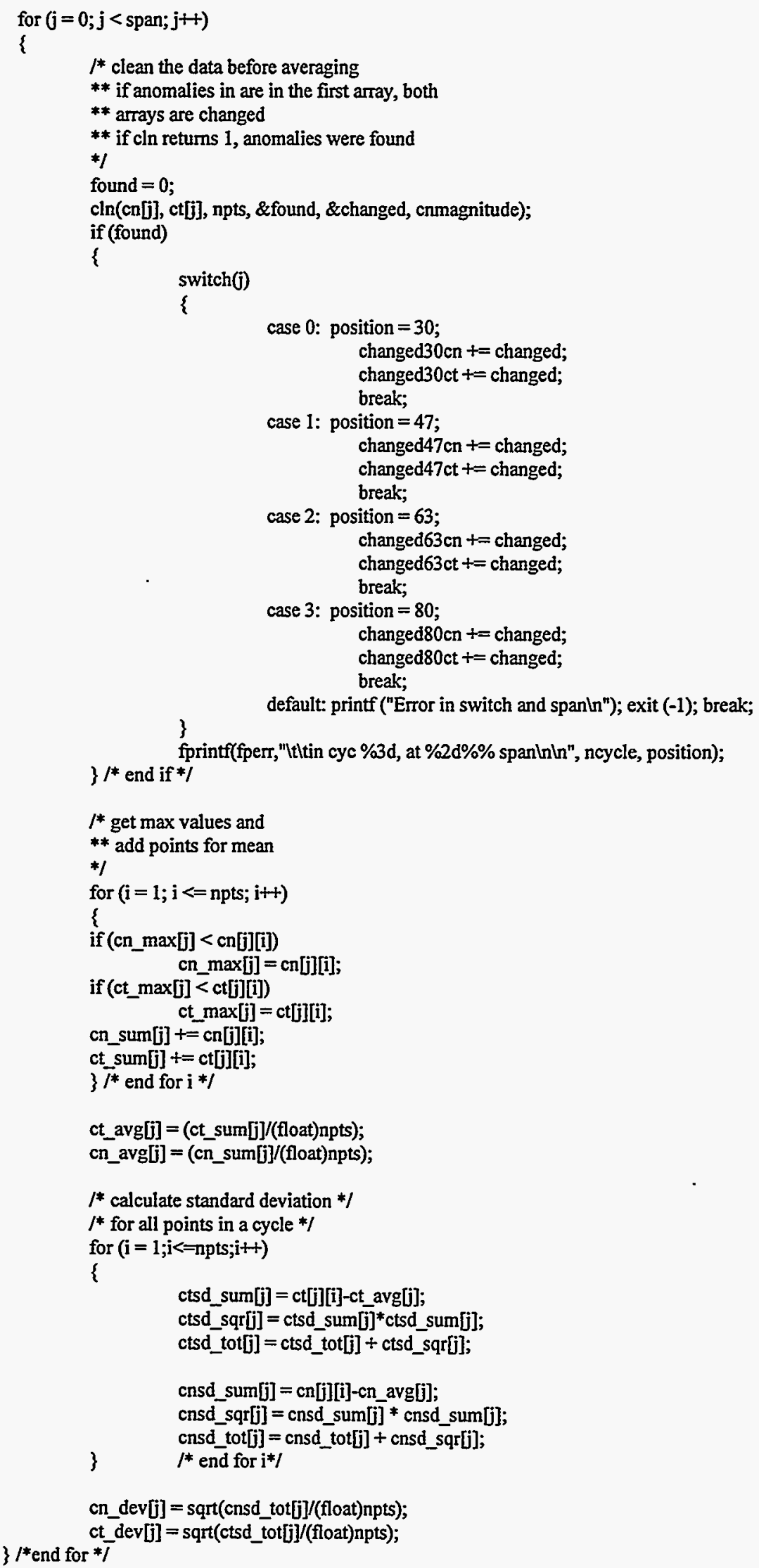


/* print cycle data *I

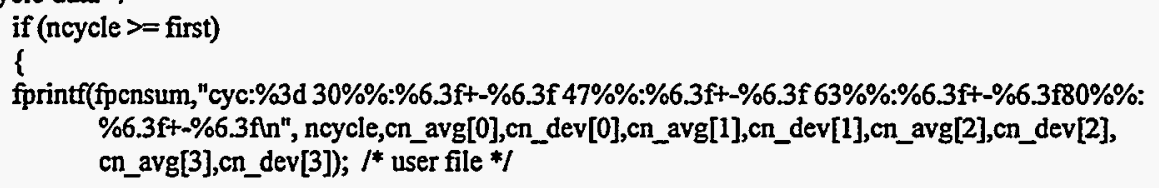
$\% 6.3 \mathrm{f}+\% 66.3 \mathrm{fn} n$, ncycle,cn_avg[0],cn_dev[0],cn_avg[1],cn_dev[1],cn_avg[2],cn_dev[2], cn_avg[3],cn_dev[3]); /* user file */

fprintf(fpctsum,"cyc:\%3d 30\%\%:\%6.3f+-\%6.3f 47\%\%:\%6.3f+-\%6.3f 63\%\%:\%6.3f+-\%6.3f 80\%\%: $\% 6.3 \mathrm{ft}-\% 6.3 \mathrm{fn} "$ ", ncycle,ct_avg[0],ct_dev[0],ct_avg[1],ct_dev[1],ct_avg[2],ct_dev[2], ct_avg[3],ct_dev[3]); /* user file */

fprintf(fpmaxsum,"cyc:\%3d cn30\%\%:\%6.3f cn $47 \% \%: \% 6.3 \mathrm{f}$ cn63\%\%:\%6.3f cn80\%\%:\%6.3f ct30\%\%: $\% 6.3 \mathrm{f}$ ct47\%\%:\%6.3f ct63\%\%:\%6.3f ct80\%\%:\%6.3f in", ncycle,cn_max[0],cn_max[1], cn_max[2],cn_max[3],ct_max[0],ct_max[1],ct_max[2],ct_max[3]); $j^{*}$ user file ${ }^{*} /$

$/ *$ don't print lst cycle since it isn't complete*/

if (ncycle ! $=1$ )

\{

fprintf(fpctout, " $\% 3 d \% 6.3 f \% 6.3 \mathrm{f} \% 6.3 \mathrm{f} \% 6.3 \mathrm{f} \% 6.3 \mathrm{f} \% 6.3 \mathrm{f} \% 6.3 \mathrm{f} \% 6.3 \mathrm{fln}$ ", ncycle,ct_avg[0], ct_dev[0],ct_avg[1],ct_dev[1],ct_avg[2],ct_dev[2],ct_avg[3],ct_dev[3]); $/ *$ data file *I

fprintf(fpcnout, "\%3d \%6.3f \%6.3f \%6.3f \%6.3f \%6.3f \%6.3f \%6.3f \%6.3fun", ncycle,cn_avg[0], cn_dev[0],cn_avg[1],cn_dev[1],cn_avg[2],cn_dev[2],cn_avg[3],cn_dev[3]); $I^{*}$ data file *I

fprintf(fpmaxout, "\% $\%$ d $\% 6.3 \mathrm{f} \% 6.3 \mathrm{f} \% 6.3 \mathrm{f} \% 6.3 \mathrm{f} \% \overline{6.3 \mathrm{f}} \% 6.3 \mathrm{f} \% 6.3 \mathrm{f} \% 6 . \overline{\mathrm{f}} \mathrm{\textrm {h }}$ ", ncycle,cn_max [0], cn_max[1],cn_max[2],cn_max[3],ct_max[0],ct_max[1],ct_max[2],ct_max[3]; $/^{*}$ data file *I $\gamma^{*}$ end if (ncycle) $* /$

\}$/ *$ end if (print cycle data) *I

\} $\quad l^{*}$ endif (a complete cycle) $* 1$

if (start)

I* a new cycle *I

$\{\quad / *$ begin if *

$I^{*}$ add data to arrays *I

nptst+;

angle[npts] $=$ az_angle;

for $(\mathrm{j}=0 ; \mathrm{j}<\operatorname{span} ; \mathrm{j}++)$

\{

$/^{*}$ cn[0][npts] for $30 \%$ span cn[1][npts] for $47 \%$ span cn[2][npts] for $63 \%$ span *I cn[3][npts] for $80 \%$ span

cn[j][npts] = mydata[cn30-j];

$3 / *$ end for $\mathrm{j} * l$

$\mathrm{ct}[\mathrm{j}][\mathrm{npts}]=$ mydata[ $[\mathrm{ct} 30-\mathrm{j}]$

$I^{*}$ endif*I

\}

count+t;

loc $=$ count $\bullet$ REC_IN_SLZE; $\quad / *$ set up next read *l

Iseek(arc_file,loc, 0 );

$l^{*}$ end while *I

$I^{*}$ print summary of changes made by $\operatorname{cin} * /$

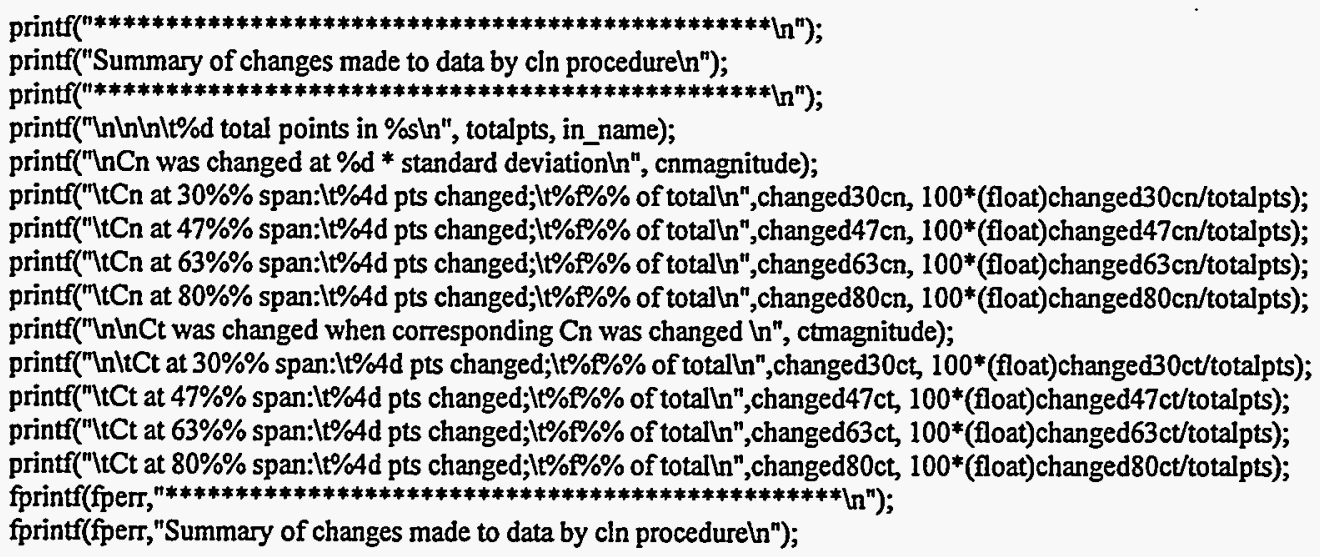




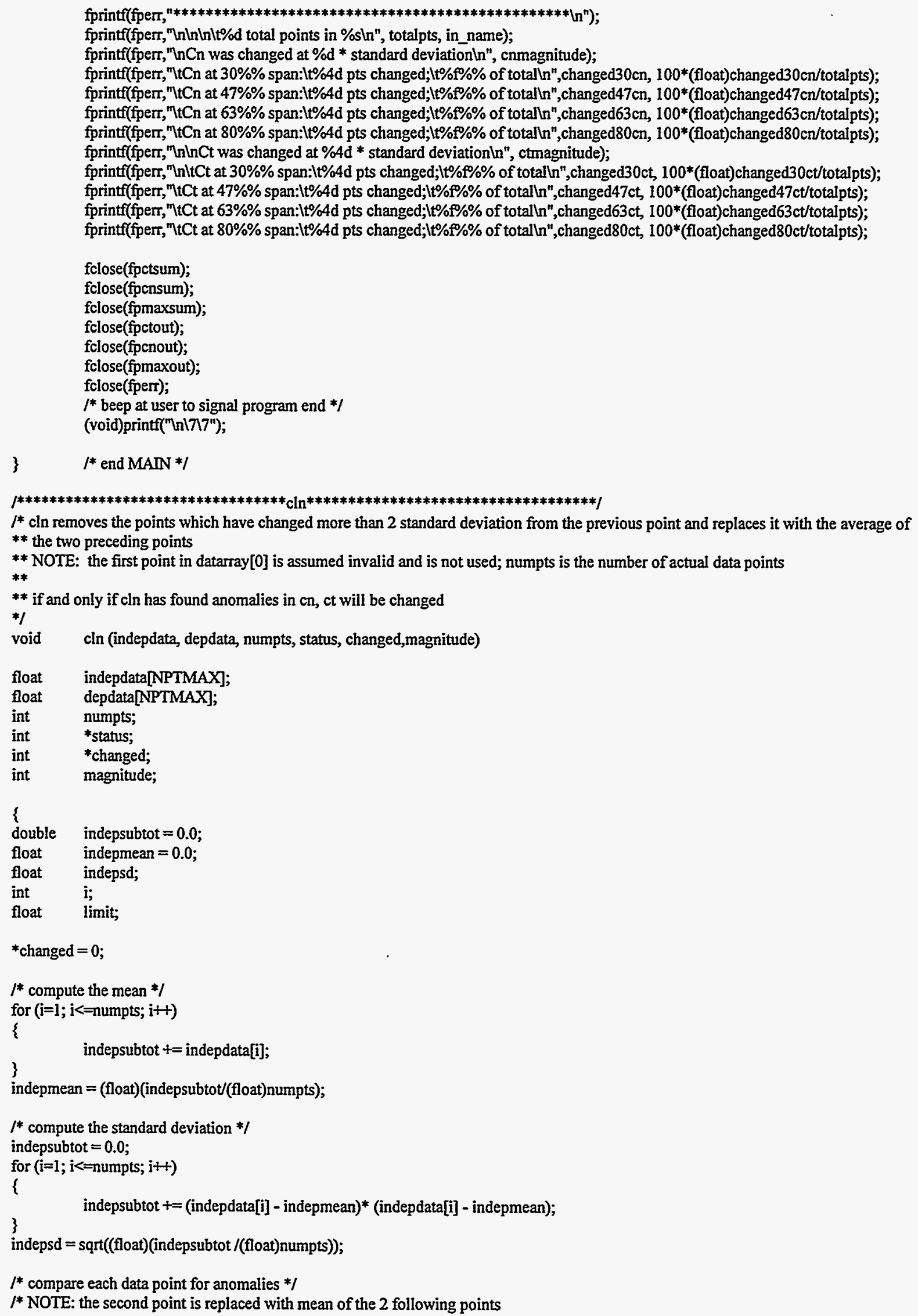


limit = magnitude * indepsd;

$\mathrm{i}=2$;

if (fabs(indepdata[i-1] - indepdata[i]) $>$ limit)

\{

fprintf(fperr,"change cn \%f ", indepdata[i]);

indepdata $[\mathrm{i}]=($ indepdata $[\mathrm{i}+1]+$ indepdata $[\mathrm{i}+2]) / 2.0$;

${ }^{*}$ status $=1$;

fprintf(fperr," to \%fln", indepdata[i]);

*changed $+=1$;

fprintf(fperr,"change ct \%f ${ }^{n}$, depdata[i]);

depdata[i] $=($ depdata $[i+1]+\operatorname{depdata}[i+2]) / 2.0$;

\} fprintf(fper," to \%fln", depdata[i]);

for $(i=3 ; i<=n u m p t s ; i+$ )

if (fabs(indepdata[i-1] - indepdata[i]) $>$ limit)

l

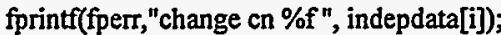

indepdata[i] = (indepdata[i-1] + indepdata[i-2])/2.0;

*status $=1$

fprintf(fperr," to \%丹n", indepdata[i]);

*changed $+=1$;

fprintf(fper, "change ct \%f ", depdata[i]);

depdata[i] $=($ depdata $[\mathrm{i}-1]+\operatorname{depdata}[\mathrm{i}-2]) / 2.0$;

\}

fprintf(fper," to \%fln", depdata[i]);

$/^{*}$ end $\operatorname{cln} * /$ 
The program AVCNCT.C also needs BEN_IO.C, READ.C, and MAIN.H to run properly. These files can be found in Appendix B.

The following three files are also necessary to properly run AVCNCT.C:

1. directory.dat:

/ust/data5

d071021

/home/windslc/tyoung/working/Avcnct

d7121

2. makefile:

\# Makefile for NREL Wind Programs 2/7/92

$\#$

HFILES $=$ main.h

PROG = avcnct

SRC = avcnct.c read.c ben_io.c

OBJ = avenct.o read.o ben_io.o

CFLAGS $=-\mathrm{g}$

$\#$

\# the default is to make PROG

\#

all: $\$\{P R O G\}$

\#

\# program depends on the .0 files (included is $-1 \mathrm{~m}$ for math.h)

$\$\{P R O G\}: \$\{O B J\}$

$c c-c \$\{O B J\}$

$\operatorname{cc} \$\{\mathrm{OBJ}\} \rightarrow \$ @-\mathrm{lm}$

\#

$\#$ the .0 files depend on the.$c$ file (implicit) and the.$h$ file

\#

$\$\{O B J\}: \$\{H F I L E S\}$

clean:

Im -f $\$\{O B J\} \$\{P R O G\}$ core

lint:

lint $\$\{S R C\}$

3. last_bin.dat

/usr/datas

d068011.hdr 
Appendix E

CNCTMAX.F 
program cnctmax

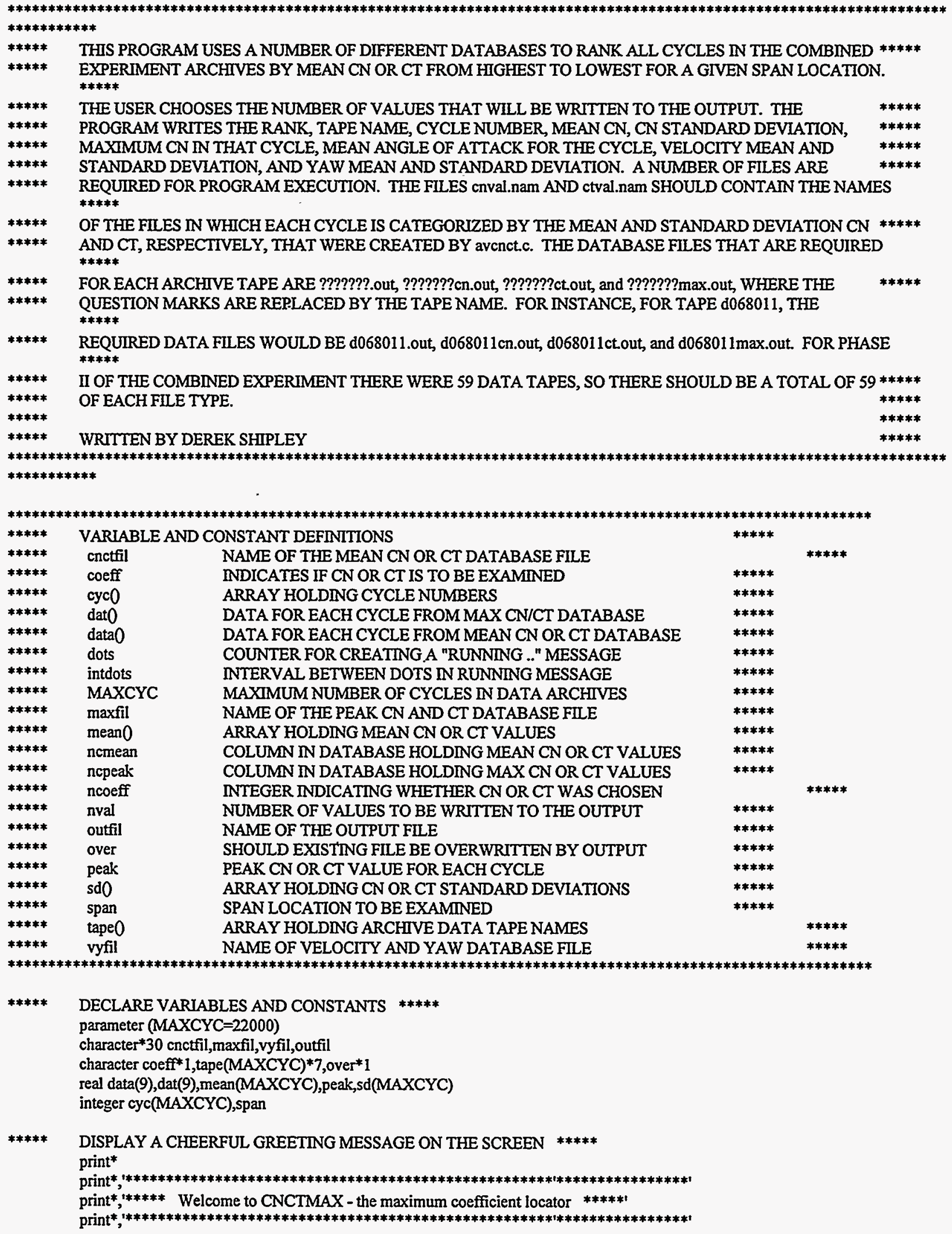

***** PROMPT THE USER FOR WHETHER CN OR CT IS TO BE EXAMINED ***** 


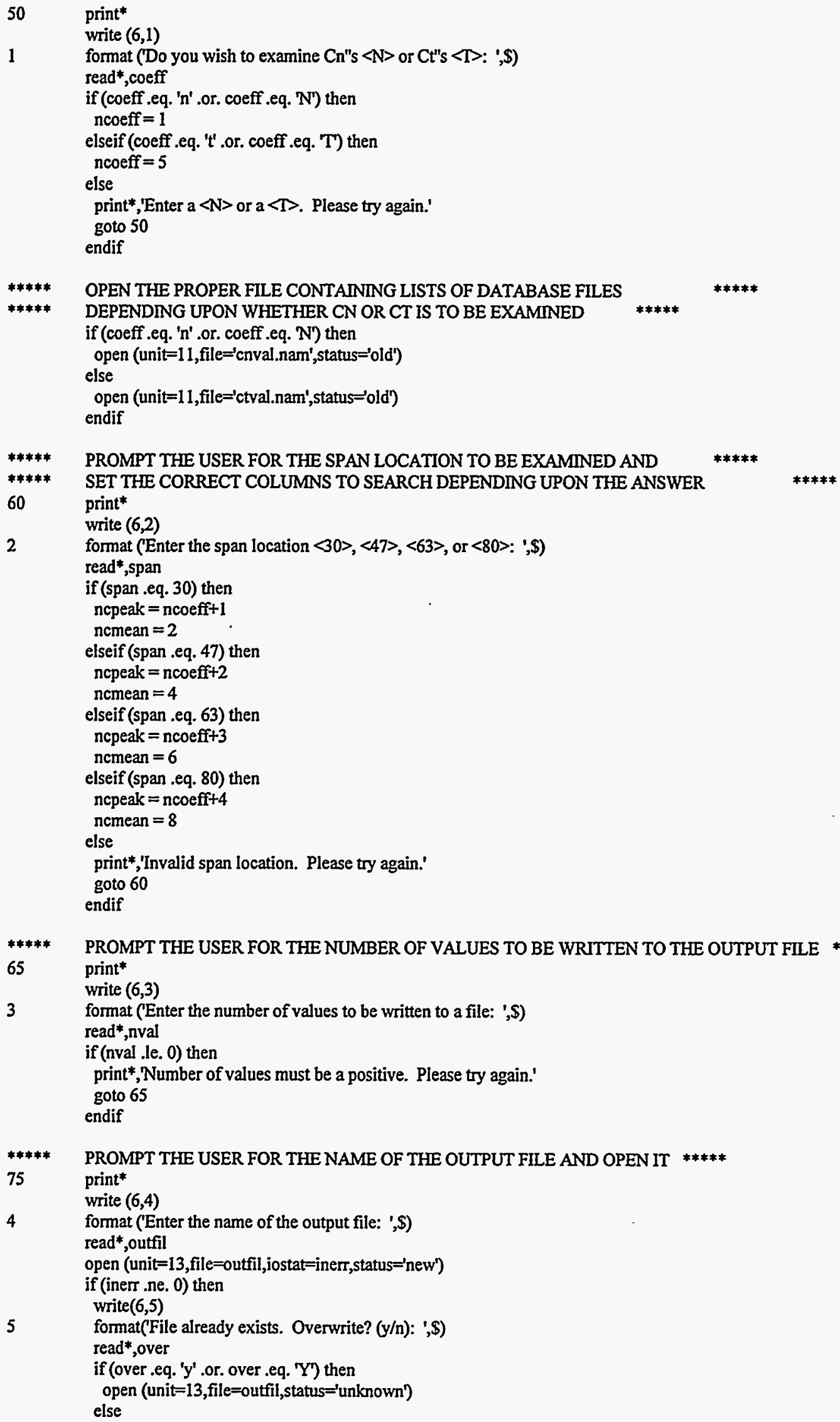

4 format ('Enter the name of the output file: '\$) read*,outfil

open (unit=13, file=outfil,iostat=inerr,status='new')

if (inerr .ne. 0 ) then

format('File already exists. Overwrite? (y/n): ',\$)

read*,over

if (over .eq. ' $y$ ' .or. over .eq. 'Y') then

open (unit=13, file=outfil,status='unknown')

else 
endif

***** WRITE HEADER INFO TO OUTPUT FILE IF CN IS BENG EXAMINED ***

if (coeff .eq. ' $n$ ' .or. coeff .eq. ' $N$ ') then

if (nval .It. 10) then

write $(13,900)$ nval,'Cn',span

elseif (nval .lt. 100) then

write $(13,901) n v a l, ' C n '$ 'span

else

write $(13,902) n v a l, ' C n '$,span

endif

write $\left(13,{ }^{*}\right)$

write $(13,905)$

***** WRITE HEADER INFO TO OUTPUT FILE IF CT IS BEING EXAMINED *****

else

if (nval .It. 10) then

write $(13,900)$ nval, 'Ct',span

elseif (nval .lt. 100) then

write $(13,901)$ nval, 'Ct',span

else

write $(13,902) \mathrm{nval}, \mathrm{Ct}$ ',span

endif

write $(13, *)$

write $(13,906)$

endif

write $(13,925)$

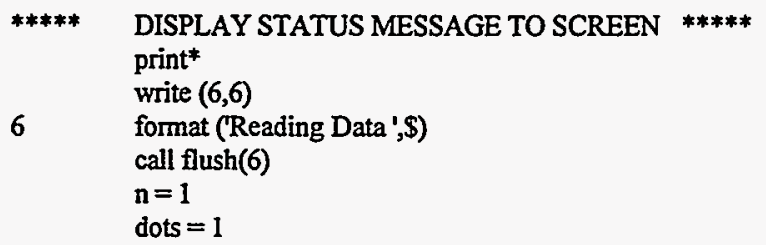

***** CALL SUBROUTINE TO SORT VALUES IN DESCENDING ORDER ***** call sort (nmax,mean,tape,cyc,sd) print*

***** BEGIN LOOP TO FIND THE PEAK CN OR CT, VELOCITY MEAN AND STANDARD DEVAIATION,

***** AND YAW MEAN AND STANDARD DEVIATION FOR EACH CYCLE TO BE WRITTEN TO THE OUTPUT ***** 
write(6,9)

***** DISPLAY STATUS MESSAGE TO THE SCREEN *****

9 format ('Getting Velocity and Yaw Values ',\$)

call flush(6)

intdots $=$ nint(nval/20.)

dots $=1$

***** BEGIN LOOP TO SEARCH FOR DESIRED CYCLES *****

do $500, n=n \max , n \max -\mathrm{nval}+1,-1$

***** OPEN MAXIMUM CN/CT DATABASE FILE *****

maxfil $=$ tape $(\mathbf{n}) / / \max$. out' $^{\prime}$

open (unit=15, file=maxfil,status $=$ 'old')

***** ADD DOTS TO THE STATUS MESSAGE TO INDICATE PROGRAM ACTIVITY *****

if (int(dots/intdots) .eq. dots/intdots) then

write $(6,7)$

call flush(6)

endif

dots $=$ dots +1

***** READ DATA UNTIL DESIRED CYCLE IS LOCATED *****

$550 \quad \operatorname{read}(15, *)(\operatorname{dat}(\mathrm{j}), \mathrm{j}=1,9)$

if (dat(1) .ne. cyc(n)) then

goto 550

else

$* * * * *$

GET PEAK CN OR CT VALUE FOR THIS CYCLE *****

peak $=$ dat(ncpeak)

close (15)

endif

***** OPEN CORRECT VELOCITY AND YAW DATABASE FILE

vyfil $=$ tape $(\mathrm{n}) / r^{\prime}$. out'

open (unit=14,file=vyfil,status $=$ 'old')

***** READ DATA UNTIL DESIRED CYCLE IS LOCATED *****

$600 \operatorname{read}\left(14,{ }^{*}\right.$, end $\left.=700\right)(\operatorname{dat}(j), j=1,9)$

if (dat(1) .eq. cyc(n)) then

***** CALL SUBOUTINE TO CALCULATE MEAN ANGLE OF ATTACK *****

call v2asub(dat(2),dat(4),span,aoa)

***** WRITE APPROPRIATE DATA TO THE OUTPUT FILE ****

write $(13,930) n m a x-n+1$, tape(n),cyc(n), mean(n),sd(n),peak,aoa,(dat(k), $k=2,5)$

close (14)

goto 700

endif

goto 600

700 continue

500 continue

***** DISPLAY STATUS MESSAGE REMINDING USER OF THE OUTPUT FILE NAME *****

print*

print*

write $(6,10)$,outfil

10 format ('Output is Contained in the File - ',230)

print*

***** FORMAT STATEMENTS FOR OUTPUT FILE ******

900 format ('The ',il,' highest mean values over a cycle for ',a2,' at ',i2, '\% span are:')

901 format ('The ',i2,' highest mean values over a cycle for ',a2,' at ',i2, $\%$ span are:')

902 format ('The ',i3,' highest mean values over a cycle for ',a2,' at ',i2, '\% span are:')

905 format ('Rank',t8,'Tape',t15,'Cyc',t20,'Mean Cn',t28,'Cn sd',t35,'Max Cn',t43,'AOA',t49,'Mean Vel',t59,'Vsd',t65,'Mean

Yaw', $176,{ }^{\prime}$ Y'sd')

906 format ('Rank',t8,'Tape',t15,'Cyc',t20,'Mean Ct',228,'Ct sd',t35,'Max Ct,'t43,'AOA',t49,'Mean Vel',t59,'Vsd',t65,'Mean Yaw',t76,'Ysd')

925

format ('$\rightarrow$

format (i3,t6,a7,t15,i3,t20,f6.3,t28,f5.3,t34,f6.2,t42,f6.3,t49,f7.3,t56,f7.3,t65,f7.3,t73,f7.3)

stop

end

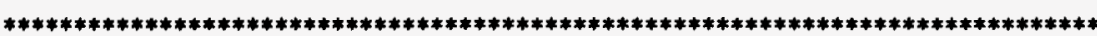

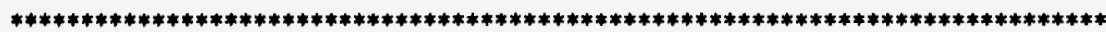


subroutine sort(n,ra,rb,rc,rd)

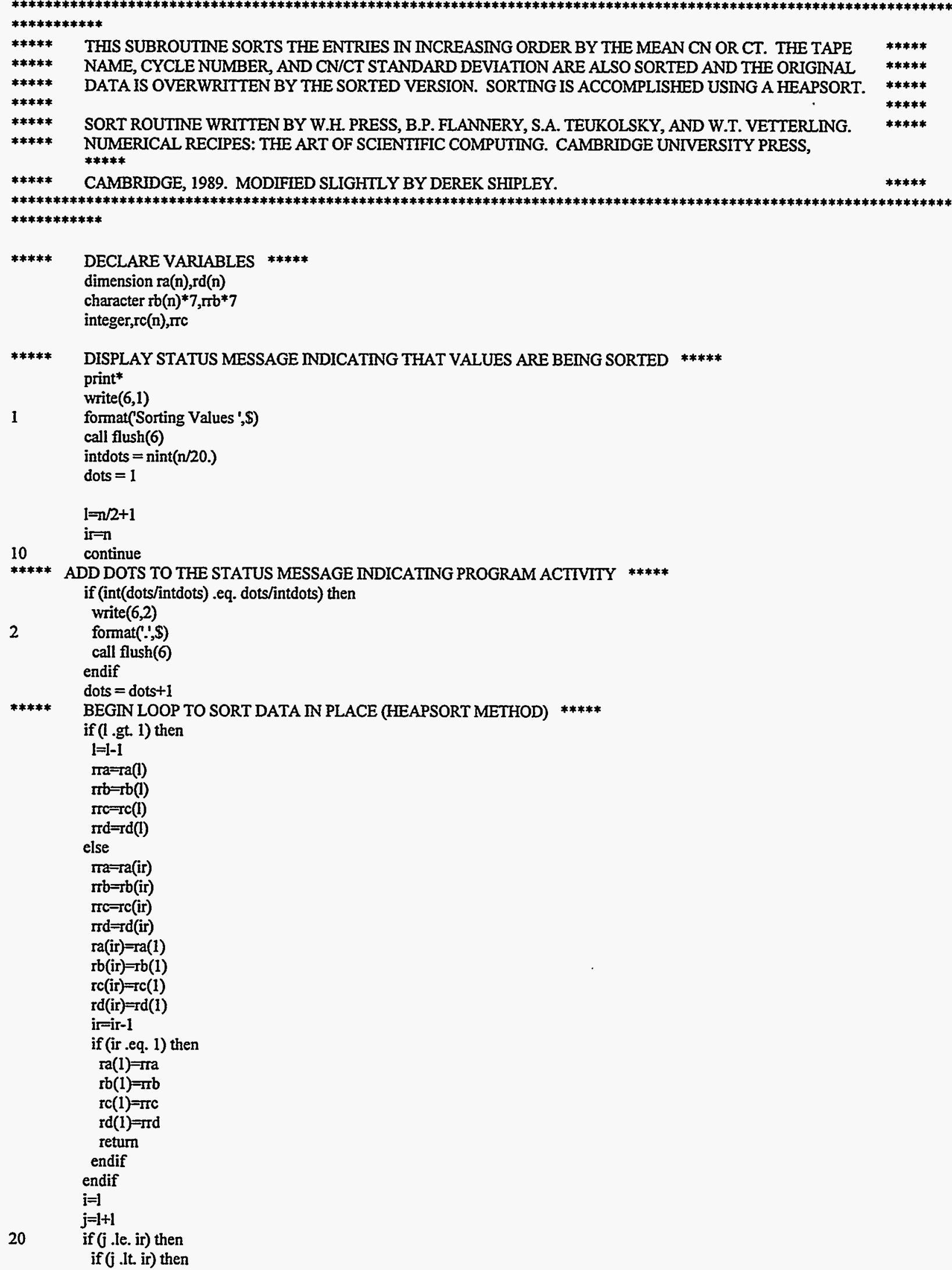

***** DISPLAY STATUS MESSAGE INDICATING THAT VALUES ARE BEING SORTED *****

print*

write $(6,1)$

1 format('Sorting Values '\$)

call flush(6)

intdots $=\operatorname{nint}(\mathrm{n} / 20$.)

dots $=1$

$1=n / 2+1$

i $=\mathbf{n}$

10 continue

***** ADD DOTS TO THE STATUS MESSAGE INDICATING PROGRAM ACTIVITY *****

if (int(dots/intdots) .eq. dots/intdots) then write $(6,2)$

2 format('.,\$)

call flush(6)

endif

dots $=$ dots +1

***** BEGIN LOOP TO SORT DATA IN PLACE (HEAPSORT METHOD) *****

if (1.gt. 1) then

$1=1-1$

пra $=\mathrm{ra}(\mathrm{l})$

$\pi b=r b(I)$

$\operatorname{mr}=\mathrm{Tc}(1)$

$\operatorname{rrd}=\mathrm{Td}(1)$

else

mara(ir)

$\mathrm{rrb}=\mathrm{rb}(\mathrm{ir})$

rrc=rc(ir)

rrd=d(ir)

$\mathrm{ra}(\mathrm{ir})=\mathrm{ra}(1)$

$\mathrm{rb}(\mathrm{ir})=\mathrm{rb}(\mathrm{l})$

$\mathrm{rc}(\mathrm{ir})=\mathrm{rc}(1)$

$\mathrm{rd}(\mathrm{ir})=\mathrm{rd}(\mathrm{l})$

ir=ir-1

if (ir .eq. 1) then

$\mathrm{ra}(1)=\mathrm{ra}$

$r b(1)=\pi b$

$\mathrm{rc}(\mathrm{I})=\pi \mathrm{rc}$

$r d(1)=$ rrd

return

endif

endif

$\mathrm{i}=1$

20 if $(j . l e$. ir $)$ then

if ( $j$.It. ir) then 

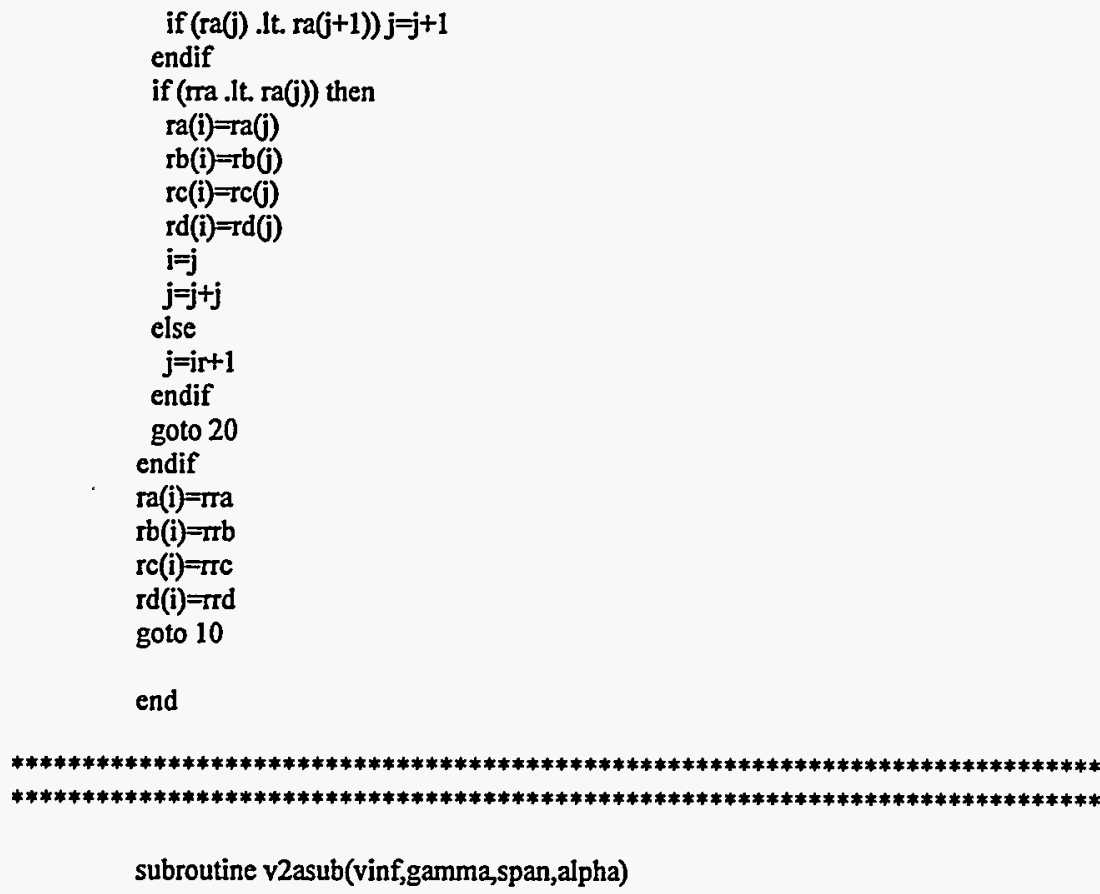

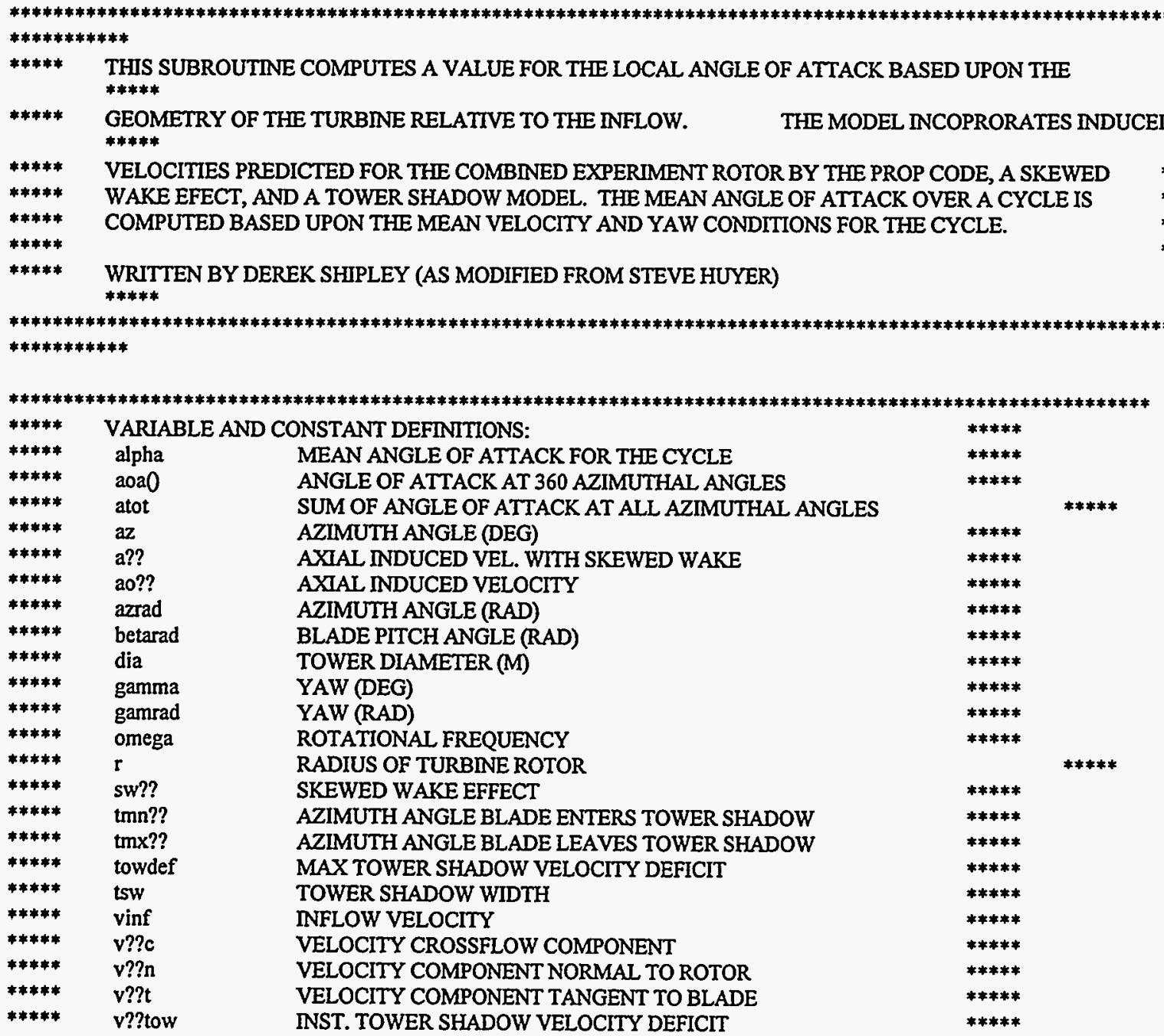




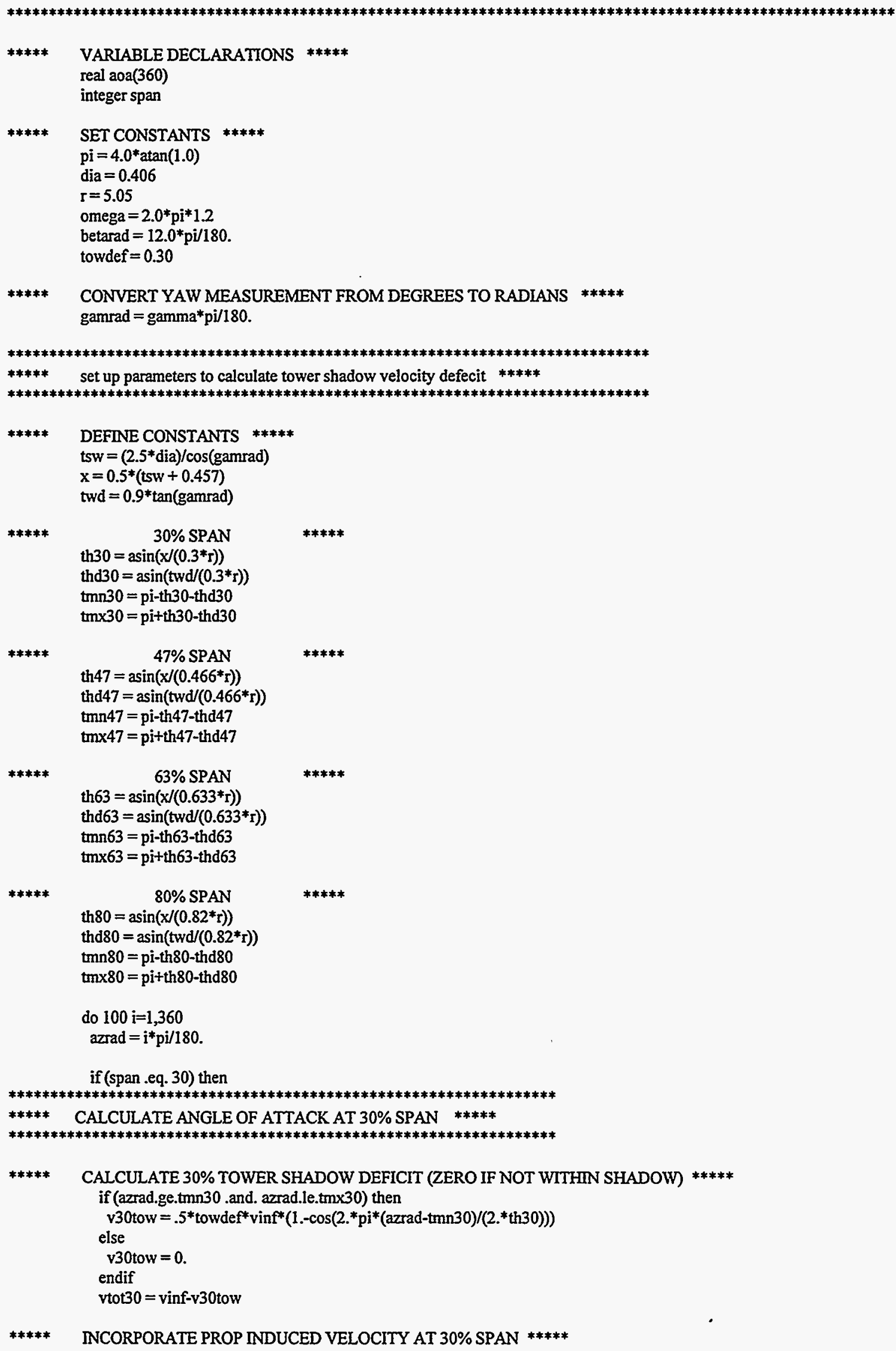


$2030=1 /\left(.000360046^{*}\right.$ vtot30**4-.0206024*vtot $30 * * 3+.415013^{*}$ vtot30**2-1.32587*vtot30+3.16774)

***** CALCULATE SKEWED WAKE EFFECT AT 30\% SPAN *****

$\mathrm{sw} 30=\left(1+15^{*} \mathrm{pi} / 32 * \mathrm{sqr}((1-\cos (\mathrm{gamrad})) /(1+\cos (\mathrm{gamrad}))) * .30 * \sin (\mathrm{azrad})\right)$

$230=2030 *$ sw 30

***** CALCULATE 30\% SPAN VELOCITY COMPONENTS AND ANGLE OF ATTACK *****

$\mathrm{v} 30 \mathrm{n}=\mathrm{vtot} 30 *(1-\mathrm{a} 30)^{*} \cos (\mathrm{gamrad})$

$\mathrm{v} 30 \mathrm{c}=-\mathrm{vtot} 30^{*} \sin (\mathrm{gamrad})$

$\mathrm{v} 30 \mathrm{t}=\left(0.30^{*} \mathrm{r}^{*}\right.$ omega $)+\mathrm{v} 30 \mathrm{c}^{*} \cos (\mathrm{azrad})$

$\mathrm{aoa}(\mathrm{i})=(\operatorname{atan}(\mathrm{v} 30 \mathrm{n} / \mathrm{v} 30 \mathrm{t})-\text { betarad })^{*} 180.0 / \mathrm{pi}$

elseif(span .eq. 47) then

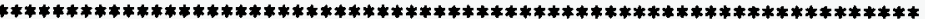

***** CALCULATE ANGLE OF ATTACK AT 47\% SPAN *****

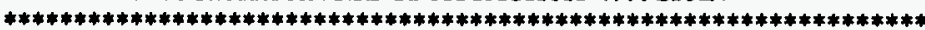

***** CALCULATE 47\% TOWER SHADOW DEFICIT (ZERO IF NOT WITHIN SHADOW) *****

if (azrad.ge.tmn47 and. azrad.le.tmx47) then

$v 47$ tow $=.5^{*}$ towdef* $\operatorname{vinf}^{*}\left(1 .-\cos \left(2 . .^{*} \mathrm{pi}^{*}(\right.\right.$ azrad-tmn 47$) /\left(2 .{ }^{*}\right.$ th 47$\left.\left.)\right)\right)$

else

v47tow $=0.0$

endif

vtot $47=$ vinf-v47tow

***** INCORPORATE PROP INDUCED VELOCITY AT 47\% SPAN *****

a $047=1 /(.000653402 *$ vtot $47 * 4-.0426646 *$ vtot $47 * * 3+1.02377 *$ vtot $47 * * 2-8.33547 *$ vtot $47+28.3554)$

***** CALCULATE SKEWED WAKE EFFECT AT 47\% SPAN *****

sw47 $=(1+15 *$ pi/32*sqrt $((1-\cos ($ gamrad $)) /(1+\cos ($ gamrad $))) * .466 * \sin ($ azrad $))$

$\mathrm{a} 47=\mathrm{a} 047^{*} \mathrm{sw} 47$

***** CALCULATE 47\% SPAN VELOCITY COMPONENTS AND ANGLE OF ATTACK *****

$\mathrm{v} 47 \mathrm{n}=\mathrm{vtot47*}(1-\mathrm{a} 47) * \cos$ (gamrad)

$v 47 \mathrm{c}=-\mathrm{vtot} 47 * \sin (\mathrm{gamrad})$

$\mathrm{v} 47 \mathrm{t}=\left(0.466^{*} \mathrm{r}^{*}\right.$ omega $)+\mathrm{v} 47 \mathrm{c}^{*} \cos (\mathrm{azrad})$

$\mathrm{aoa}(\mathrm{i})=(\operatorname{atan}(\mathrm{v} 47 \mathrm{n} / \mathrm{v} 47 \mathrm{t})-$ betarad $) * 180.0 / \mathrm{pi}$

elseif (span .eq. 63) then

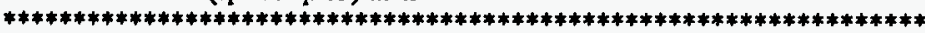

***** CALCULATE ANGLE OF ATTACK AT 63\% SPAN ******

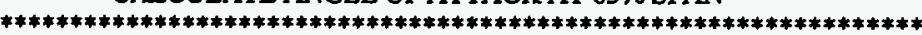

***** CALCULATE 63\% TOWER SHADOW DEFICIT (ZERO IF NOT WITHIN SHADOW) *****

if (azrad.ge.tmn63 .and. azrad.le.tmx63) then

v63tow $=.5 *$ towdef $\operatorname{vinf}^{*}\left(1 .-\cos \left(2 .{ }^{*} \mathrm{pi}^{*}(\operatorname{azrad}-\operatorname{tmn} 63) /(2 . *\right.\right.$ th63) $\left.)\right)$

else

$v 63$ tow $=0.0$

endif

vtot63 $=$ vinf-v63tow

***** INCORPORATE PROP INDUCED VELOCITY AT 63\% SPAN *****

a063 $=1 /\left(.000857403^{*}\right.$ vtot63**4-.0580386*vtot63**3+1.45897*vtot63**2-14.2769*vtot $\left.63+55.6623\right)$

***** CALCULATE SKEWED WAKE EFFECT AT 63\% SPAN *****

sw63 $=(1+15 *$ pi/32* $\operatorname{sqr}((1-\cos ($ gamrad $)) /(1+\cos ($ gamrad $))) * .633 * \sin ($ azrad $))$

$a 63=2063 *$ sw63

***** CALCULATE 63\% SPAN VELOCITY COMPONENTS AND ANGLE OF ATTACK *****

v63n $=$ vtot $63 *(1-a 63) * \cos$ (gamrad)

$v 63 \mathrm{c}=-\mathrm{vtot} 63 * \sin (\mathrm{gamrad})$

$v 63 t=\left(0.633 * r^{*}\right.$ omega $)+v 63 c^{*} \cos (a z r a d)$

$\mathrm{aoa}(\mathrm{i})=(\operatorname{atan}(\mathrm{v} 63 \mathrm{n} / \mathrm{v} 63 \mathrm{t})-$ betarad $) * 180 / \mathrm{pi}$

elseif (span .eq. 80) then

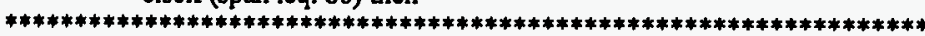

***** CALCULATE ANGLE OF ATTACK AT 80\% SPAN 


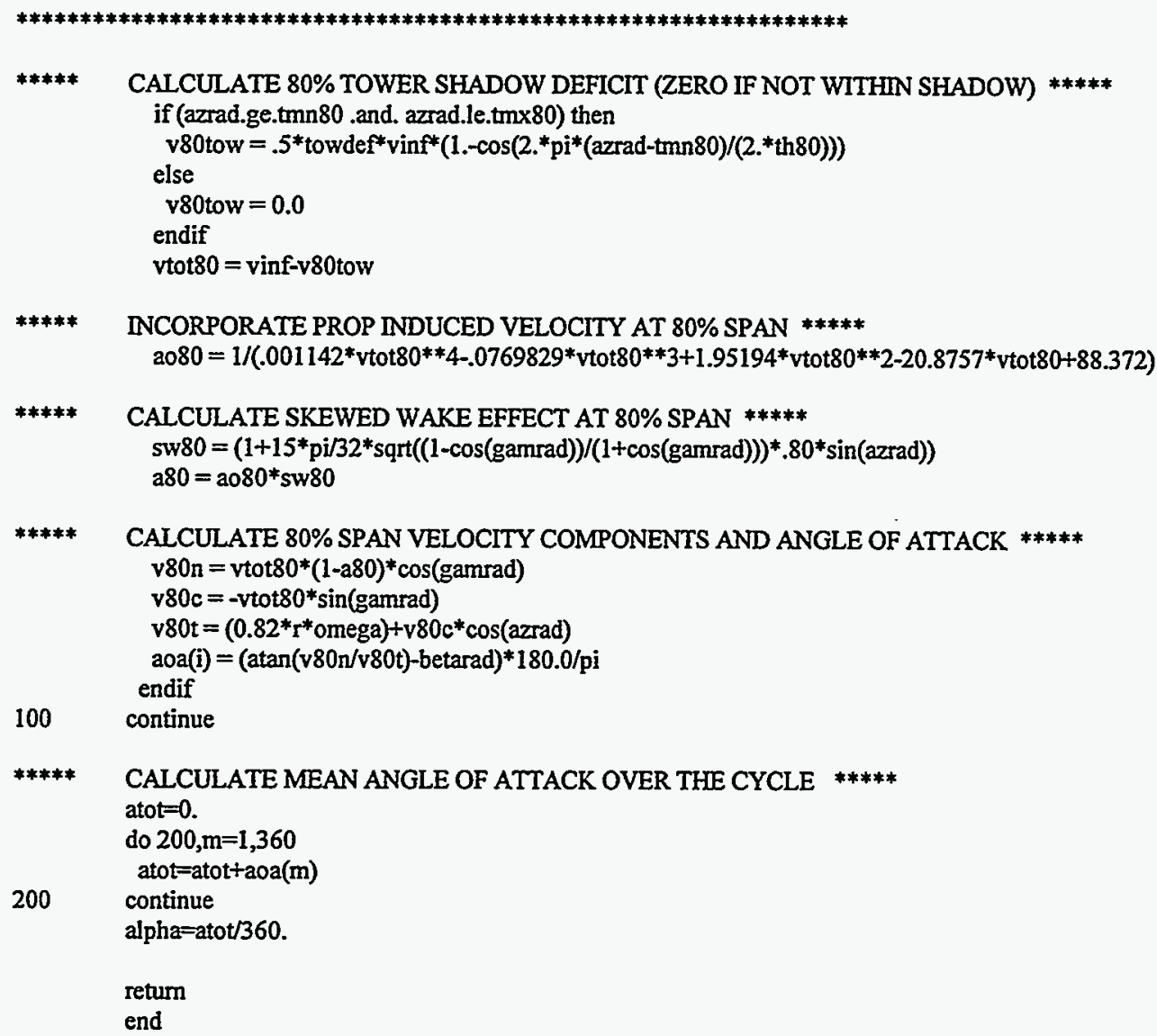

***** CALCULATE MEAN ANGLE OF ATTACK OVER THE CYCLE *****

atot $=0$.

do $200, \mathrm{~m}=1,360$

atot $=$ atot + aoa $(\mathrm{m})$

200 continue

alpha $=\mathrm{atot} / 360$.

return

end 


\section{Appendix F}

\section{DSTALLTAPE.F}


program dstalltape

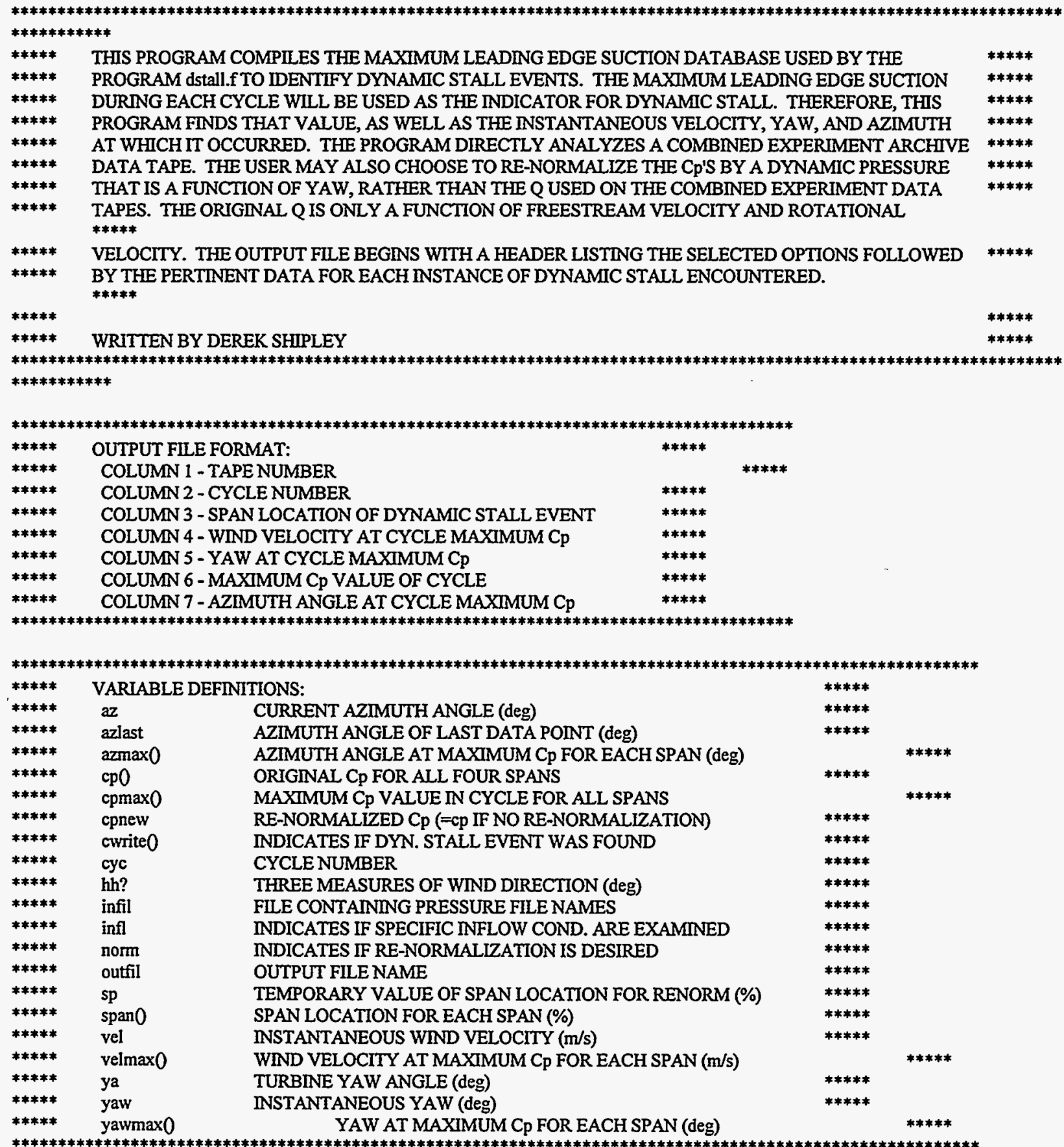

dimension bin(239)

***** VARIABLE DECLARATIONS *****

character infil $* 30$,outfil $* 30$,norm*1

real $\mathrm{cp}(4), \mathrm{cpmax}(4), \mathrm{velmax}(4), y a w \max (4), \operatorname{azmax}(4)$

integer cyc,cwrite(4),span(4),sp

***** SET CONSTANTS *****

MAXPTS $=445$

$\operatorname{span}(1)=30$

$\operatorname{span}(2)=47$ 
$\operatorname{span}(3)=63$

$\operatorname{span}(4)=80$

*****

print

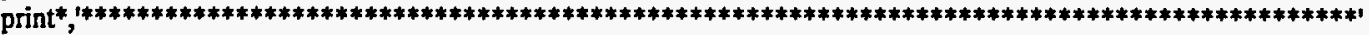

print ${ }^{*}, * * * *$ WELCOME TO DSTALLTAPE - THE MAX LEADING EDGE SUCTION LOCATOR *****!

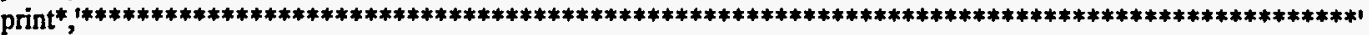

\#\#\#\#*

100

PROMPT THE USER FOR THE NAME OF THE INPUT FILE AND OPEN IT *****

print*

write $(6,1)$

1 format ('Enter the name of the input file (including path): '\$\$)

read'(a30)', infil .

open (unit=I l,recl=956,form='unformatted', file=infil,iostat=iner, access='direct',status='old')

if (inerr .ne. 0) then

print*, 'File does not exist. Please try again.'

goto 100

endif

do $125, \mathrm{i}=1,30$

if (infil(i:i+1) .eq. ' $d 0^{\prime}$ ) then

infil=infil(i:i+6)

goto 135

endif

125 continue

135 continue

***** ASK THE USER IF THEY WANT TO RE-NORMALIZE THE Cp'S *****

print*

write $(6,4)$

4 format ('Do you wish to re-normalize the Cp"s with a yaw dependent $q$ ? $(y / n):$ ',\$)

read*, norm

***** PROMPT THE USER FOR THE OUTPUT FILE NAME AND OPEN FILE

200 print*

write $(6,3)$

3 format ('Enter the name of the output file: ',\$)

read'(a30)', outfil

open (unit $=13$, file $=$ outfil,status $=$ 'unknown')

***** CREATE OUTPUT FILE HEADER *****

write $(13,9000)$

if (norm .eq. ' $y$ ' .or. norm .eq. ' 'Y') then

write $(13,9100)$

else

write $(13,9200)$

endif

write $(13,9300)$

write $(13,9400)$

write $(13,9500)$

write $(13, *)$

write $(13,9600)$

write $(13,9700)$

write $(13, *)$

print*

azlast $=0$.

numpts $=0$

cyc $=1$

nfound $=0$

numrec $=1$

print*,'Examining tape ',infil

***** READ DATA FROM INPUT FILE ONE POINT AT A TIME *****

$500 \quad \operatorname{read}(11$, rec=numrec,end $=600)($ bin $(i), i=1,239)$

numpts $=$ numpts +1

$\mathrm{az}=\operatorname{bin}(57)$ 


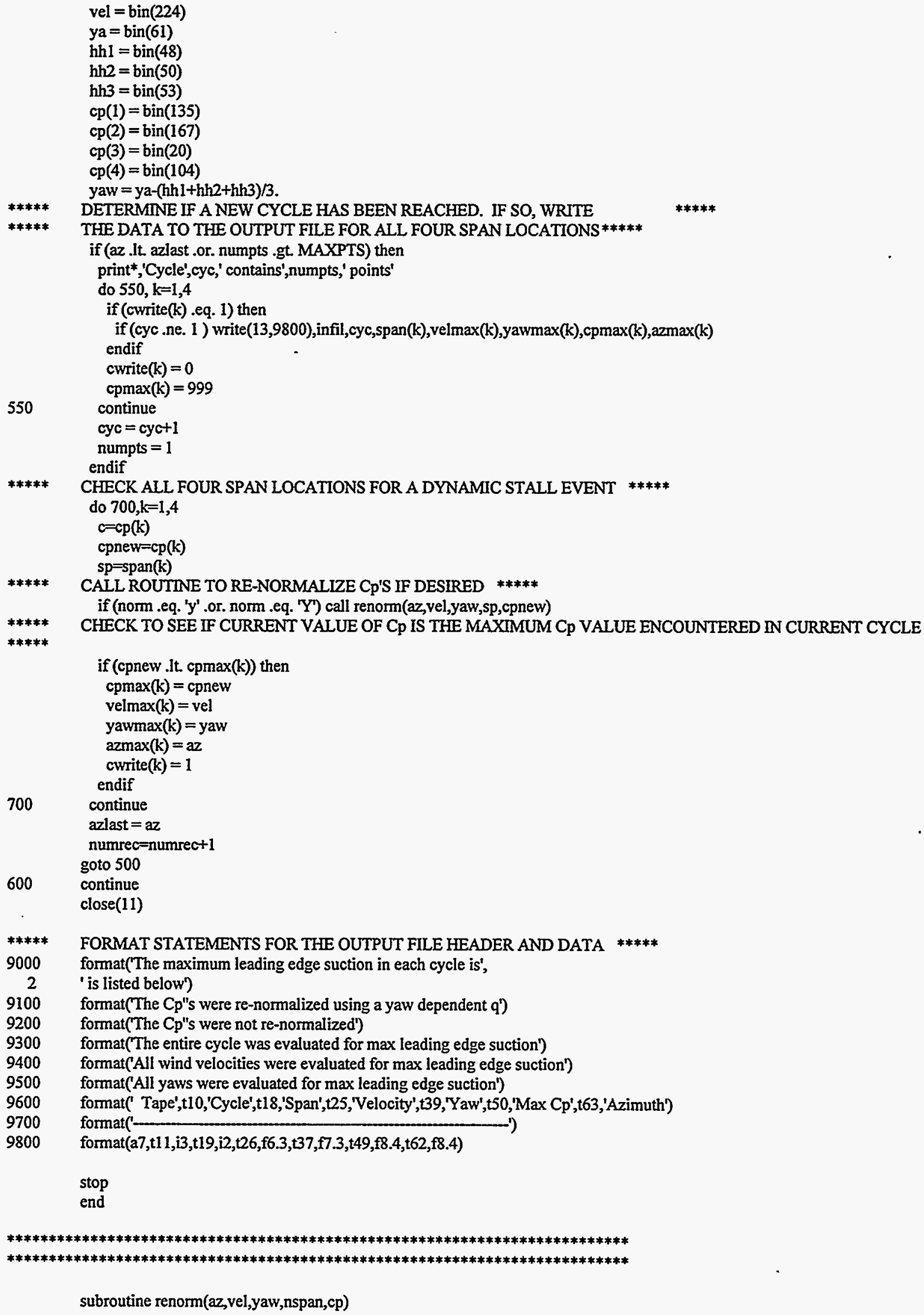

FORMAT STATEMENTS FOR THE OUTPUT FILE HEADER AND DATA *****

format('The maximum leading edge suction in each cycle is',

'is listed below')

format(The $C p$ "s were re-normalized using a yaw dependent $q$ ')

format('The Cp"s were not re-normalized')

format('The entire cycle was evaluated for max leading edge suction')

format(All wind velocities were evaluated for max leading edge suction')

format(All yaws were evaluated for max leading edge suction')

format(' Tape',t10,'Cycle',t18,'Span',t25, 'Velocity',t39,'Yaw',t50,'Max Cp',t63,'Azimuth')

format(')

format $(\mathrm{a} 7, \mathrm{t} 11, \mathrm{i} 3, \mathrm{t} 19, \mathrm{i} 2, \mathrm{t} 26, \mathrm{f6} 6.3, \mathrm{t3} 7, \mathrm{f7} .3, \mathrm{t49}, \mathrm{f8} .4, \mathrm{t} 62, \mathrm{f8} .4)$

stop

end 


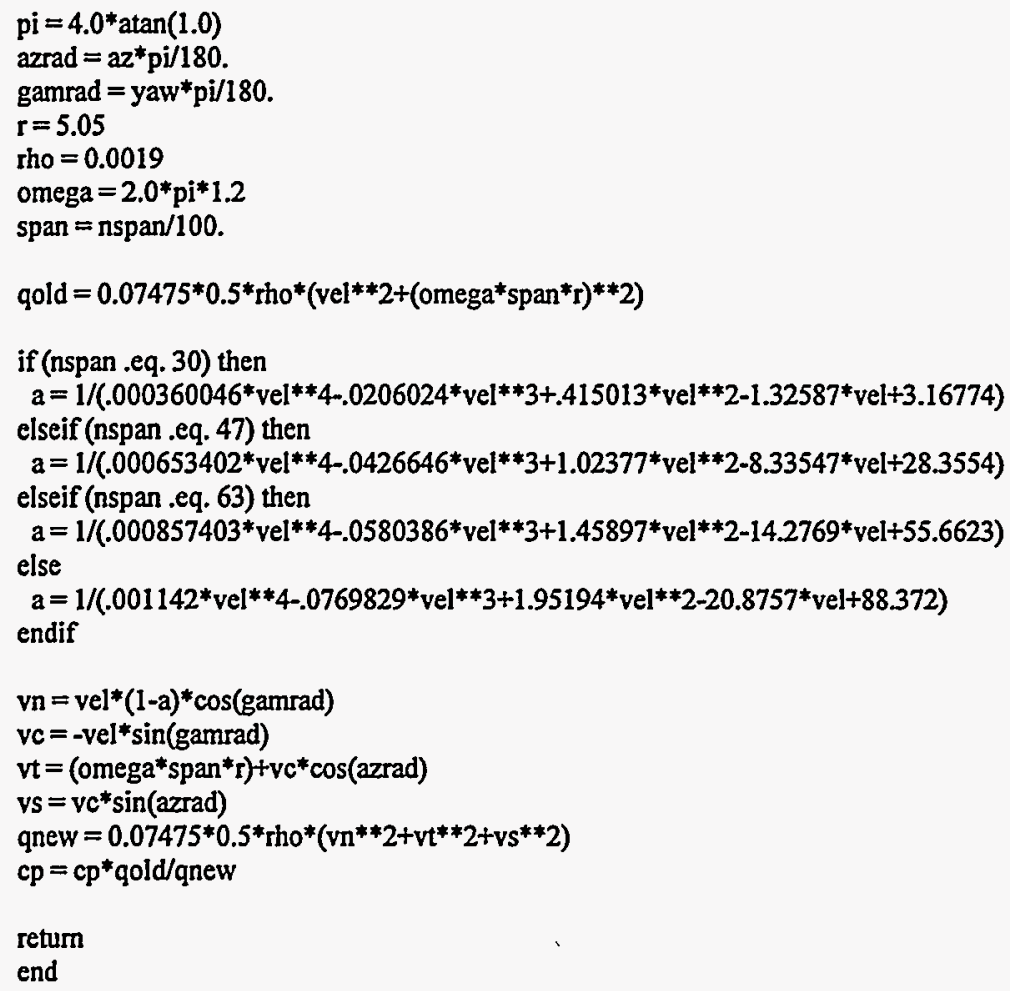




\section{Appendix G}

\section{DSTALL.F}


program dstall

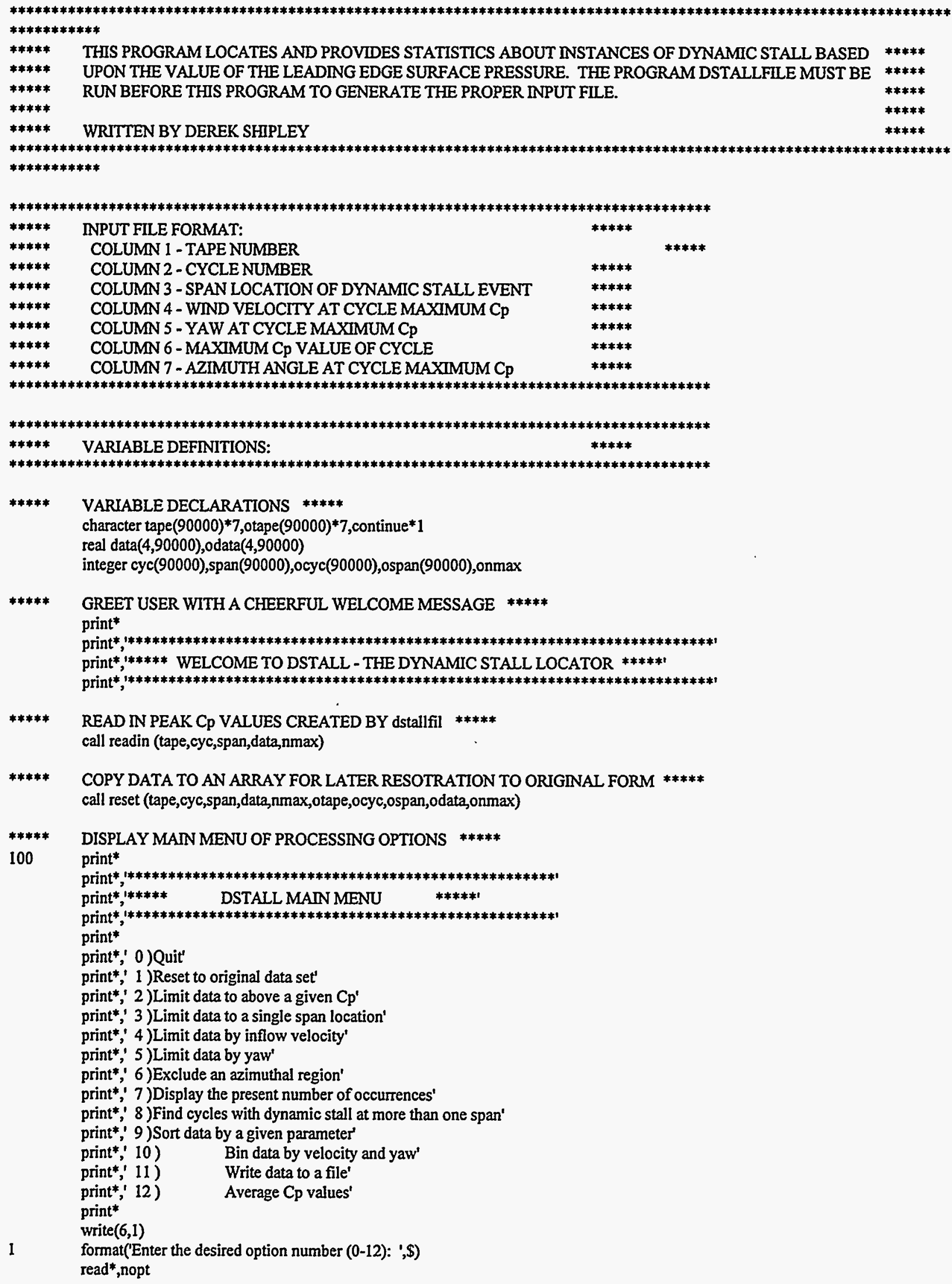


if (nopt .eq. 0 ) then goto 900

elseif (nopt .eq. 1) then

call reset (otape,ocyc,ospan,odata, onmax,tape,cyc,span,data,nmax)

print*

print 9000

write $(12,9000)$

elseif (nopt .eq. 2) then

call limcp (tape,cyc,span,data, nmax)

elseif (nopt .eq. 3) then

call limspan (tape,cyc,span,data,nmax)

elseif (nopt .eq. 4) then

call limvel (tape,cyc,span,data,nmax)

elseif (nopt .eq. 5) then

call limyaw (tape,cyc,span, data,nmax)

elseif (nopt .eq. 6) then

call limaz (tape,cyc,span,data,nmax)

elseif (nopt .eq. 7) then

print*

print 9100, nmax

write $(12,9100)$, nmax

elseif (nopt .eq. 8) then

call muitspan(tape,cyc,span,data,nmax)

elseif (nopt .eq. 9) then

call sort(tape,cyc,span,data,nmax)

elseif (nopt .eq. 10) then

call bindat(data,nmax,odata,onmax)

elseif (nopt .eq. 11) then

call writdat (tape,cyc,span,data,nmax)

elseif (nopt .eq. 12) then

call avecp (data,nmax)

else

print*, 'You entered an invalid option. Please try again.'

goto 100

endif

print*

2 format('Hit ENTER to continue: ',\$)

read'(al)',continue

goto 100

900 continue

9000 format ('Data reset to original conditions')

9100 format (The current number of occurrences is ',i5)

stop

end

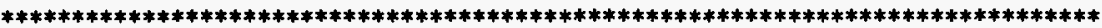

*****************************************************************************

subroutine readin(tape,cyc,span,data,nmax)

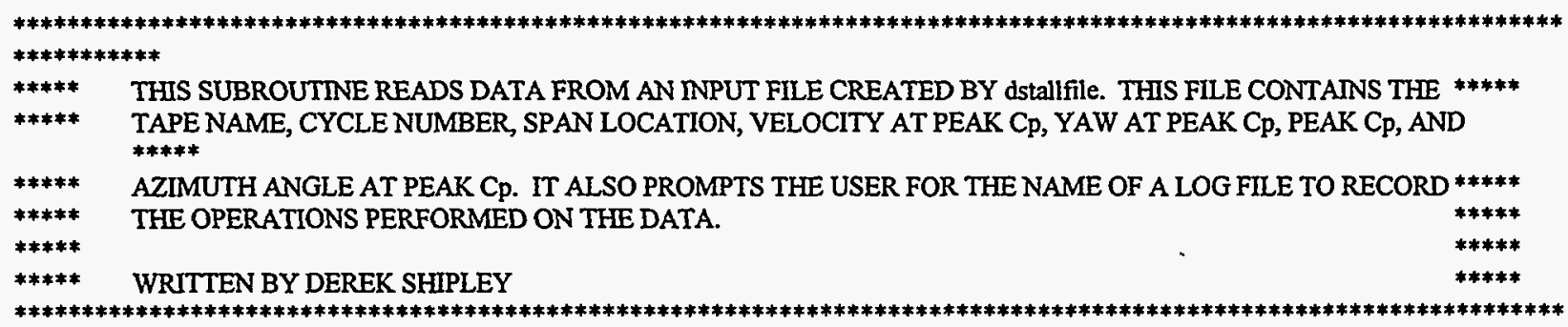




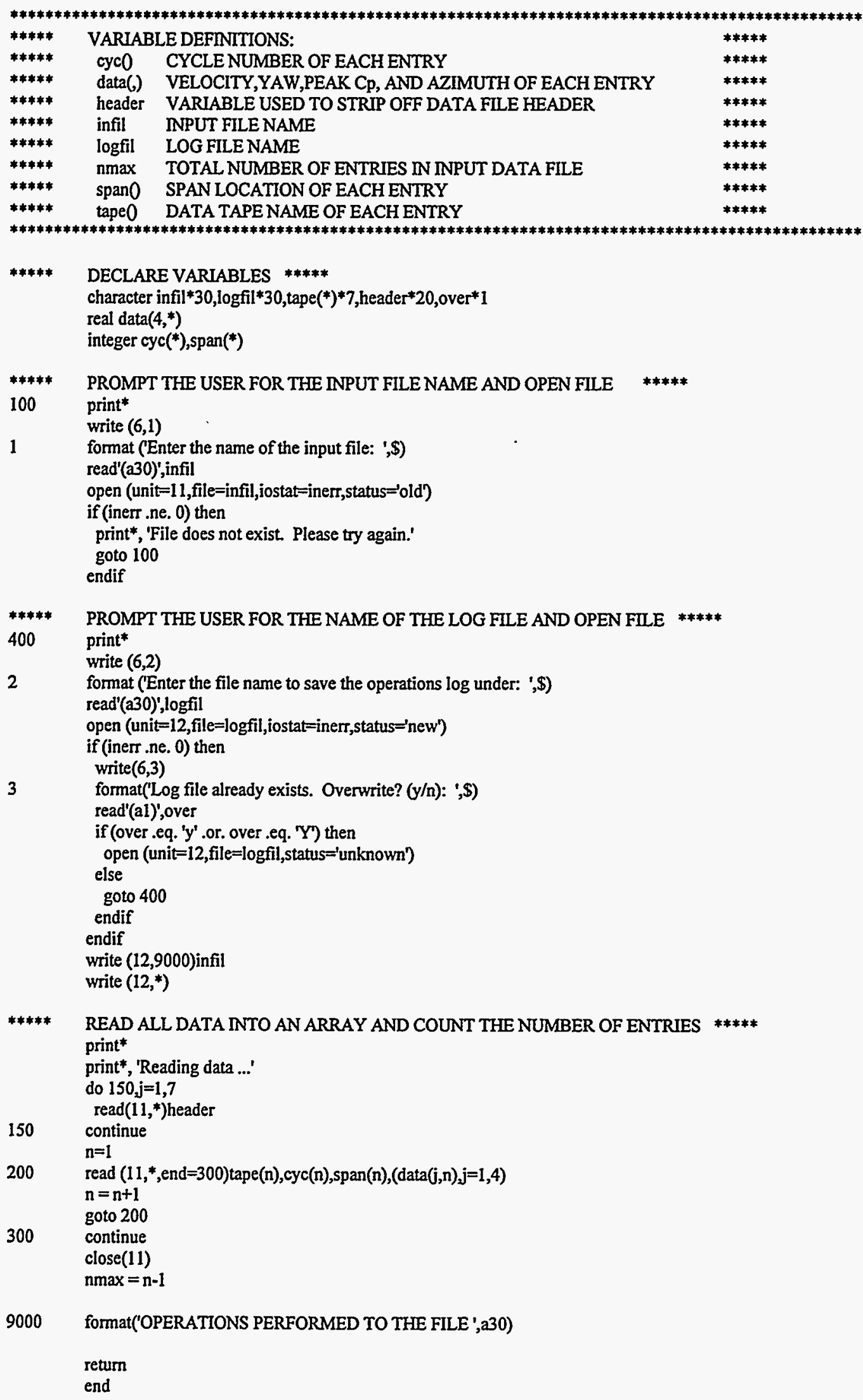

format('OPERATIONS PERFORMED TO THE FILE ',a30)

return

end 
subroutine reset(oldtape,oldcyc,oldspan,olddata,oldnmax,newtape,newcyc,newspan,newdata,newnmax)

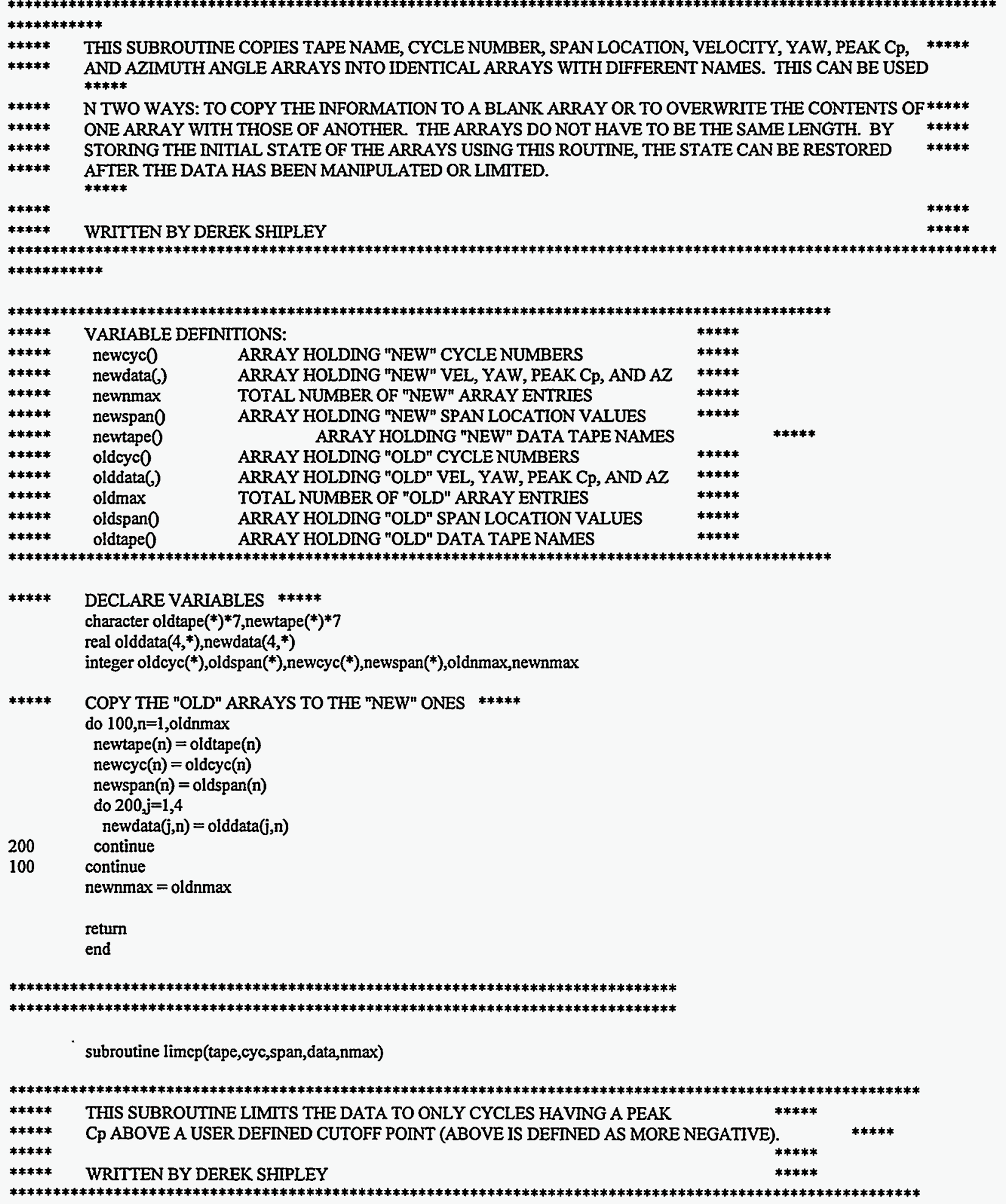




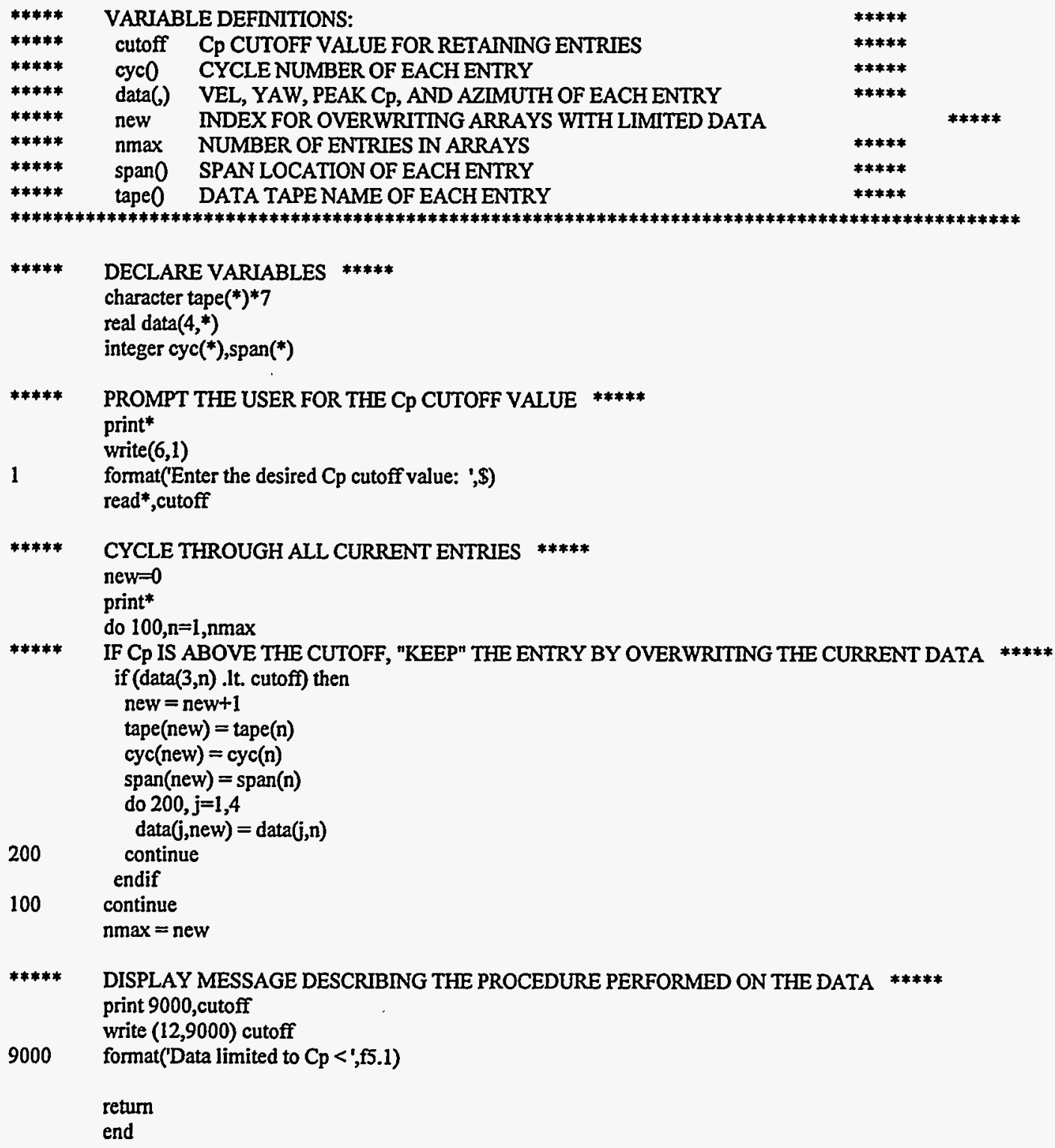




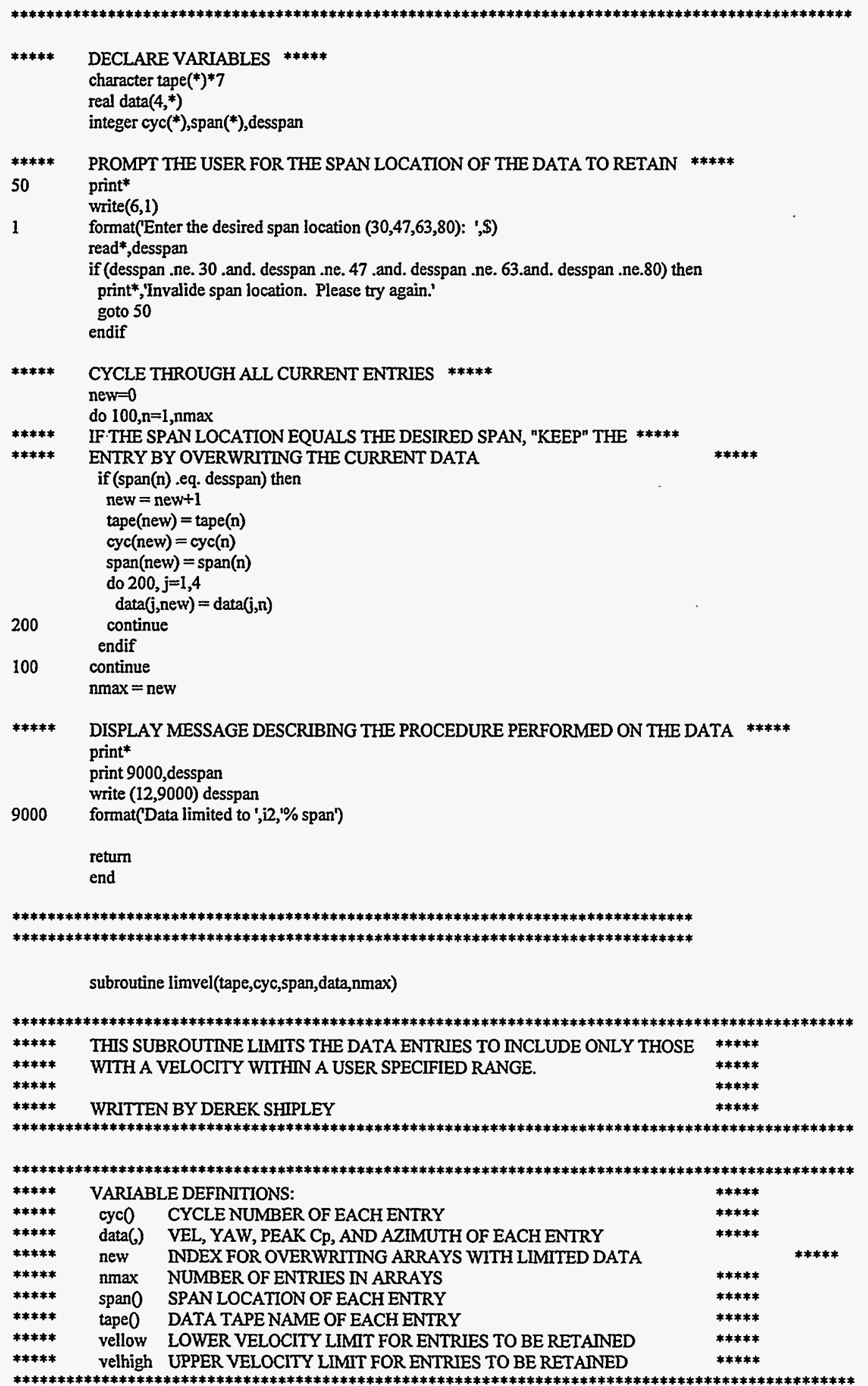




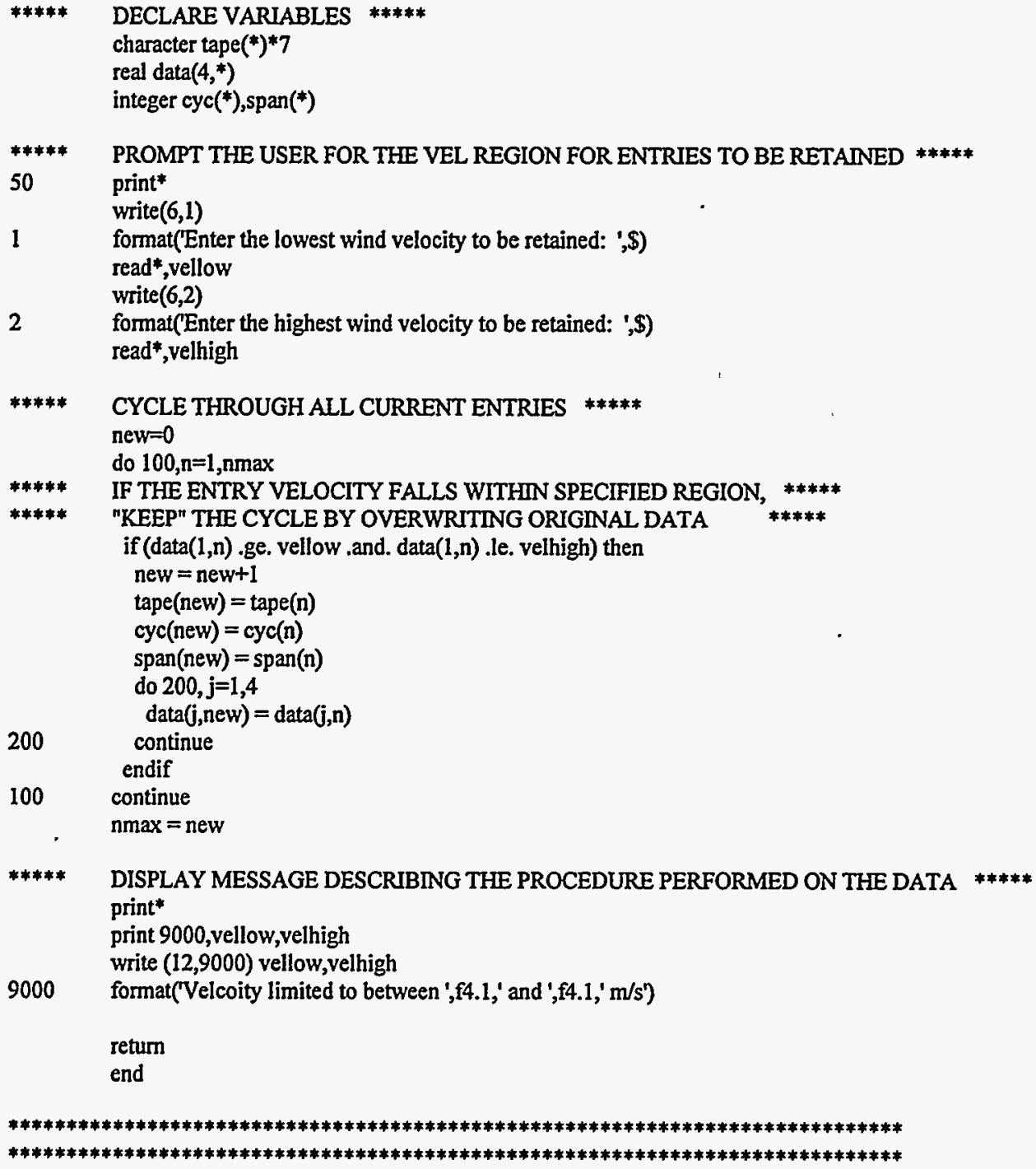


integer cyc $\left(^{*}\right), \operatorname{span}(*)$

***** PROMPT THE USER FOR THE YAW REGION FOR ENTRIES TO BE RETAINED *****

50 print*

write $(6,1)$

1 format('Enter the lowest yaw to be retained: '\$)

read*,yawlow

write $(6,2)$

2 format('Enter the highest yaw to be retained: '\$)

read*,yawhigh

***** CYCLE THROUGH ALL CURRENT ENTRIES *****

new $=0$

do $100, \mathrm{n}=1, \mathrm{nmax}$

***** IF THE ENTRY YAW FALLS WITHIN SPECIFIED REGION, *****

***** $\quad$ "KEEP" THE CYCLE BY OVERWRITING ORIGINAL DATA *****

if $($ data $(2, n)$.ge. yawlow .and. data $(2, n)$.le. yawhigh) then

new $=$ new 1

$\operatorname{tape}($ new $)=\operatorname{tape}(n)$

$\operatorname{cyc}(n e w)=\operatorname{cyc}(n)$

$\operatorname{span}($ new $)=\operatorname{span}(n)$

do $200, j=1,4$

data(j,new $)=$ data $(\mathrm{j}, \mathrm{n})$

200

continue

endif

100 continue

nmax $=$ new

***** DISPLAY MESSAGE DESCRIBING THE PROCEDURE PERFORMED ON THE DATA *****

print*

print 9000 ,yawlow,yawhigh

write $(12,9000)$ yawlow,yawhigh

9000 format('Yaw limited to between ',f5.1,' and ',f5.1,' degrees')

return

end

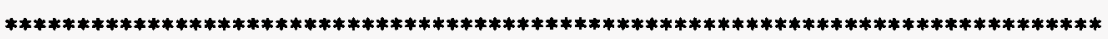

*****************************************************************************

subroutine limaz(tape,cyc,span,data,nmax)

\begin{tabular}{|c|c|c|}
\hline \#**\#* & THIS SUBROUTINE LIMITS THE DATA ENTRIES TO EXCLUDE THOSE WITH & ***** \\
\hline ***** & \multirow[t]{2}{*}{ AN AZIMUTH ANGLE THAT FALLS WITHIN A USER SPECIFIED RANGE. } & ***** \\
\hline 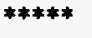 & & ***** \\
\hline ***** & WRITTEN BY DEREK SHIPLEY & ***** \\
\hline \multicolumn{3}{|c|}{ 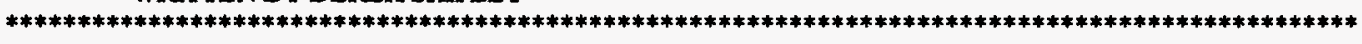 } \\
\hline \multicolumn{3}{|c|}{ 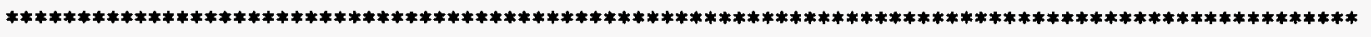 } \\
\hline **\#** & VARIABLE DEFINITIONS: & \#**** \\
\hline \#**** & LOWER AZIMUTH LIMIT FOR ENTRIES TO BE EXCLUDED & $* * * * *$ \\
\hline ***** & azhigh UPPER AZIMUTH LIMIT FOR ENTRIES TO BE EXCLUDED & ***** \\
\hline ***** & CYCLE NUMBER OF EACH ENTRY & \#\#\#** \\
\hline$\neq * \# *$ & VEL, YAW, PEAK Cp, AND AZIMUTH OF EACH ENTRY & $\neq * * * *$ \\
\hline 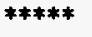 & INDEX FOR OVERWRITING ARRAYS WITH LIMITED DATA & ****** \\
\hline ***** & NUMBER OF ENTRIES IN ARRAYS & ***** \\
\hline 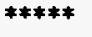 & SPAN LOCATION OF EACH ENTRY & ***** \\
\hline$* * * * *$ & DATA TAPE NAME OF EACH ENTRY & ***** \\
\hline \multicolumn{3}{|c|}{ 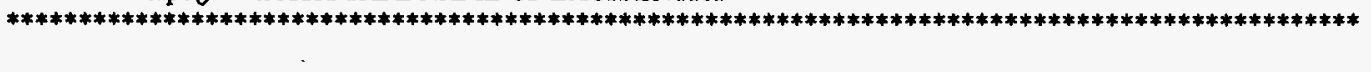 } \\
\hline$* * * * *$ & \multicolumn{2}{|l|}{$\begin{array}{l}\text { DECLARE VARIABLES } \\
\text { character tape }(*)^{*} 7 \\
\text { real data }\left(4,{ }^{*}\right) \\
\text { integer cyc }\left(^{*}\right), \operatorname{span}\left(^{*}\right)\end{array}$} \\
\hline
\end{tabular}

***** PROMPT THE USER FOR THE AZIMUTHAL REGION FOR ENTRIES TO BE EXCLUDED ****** 


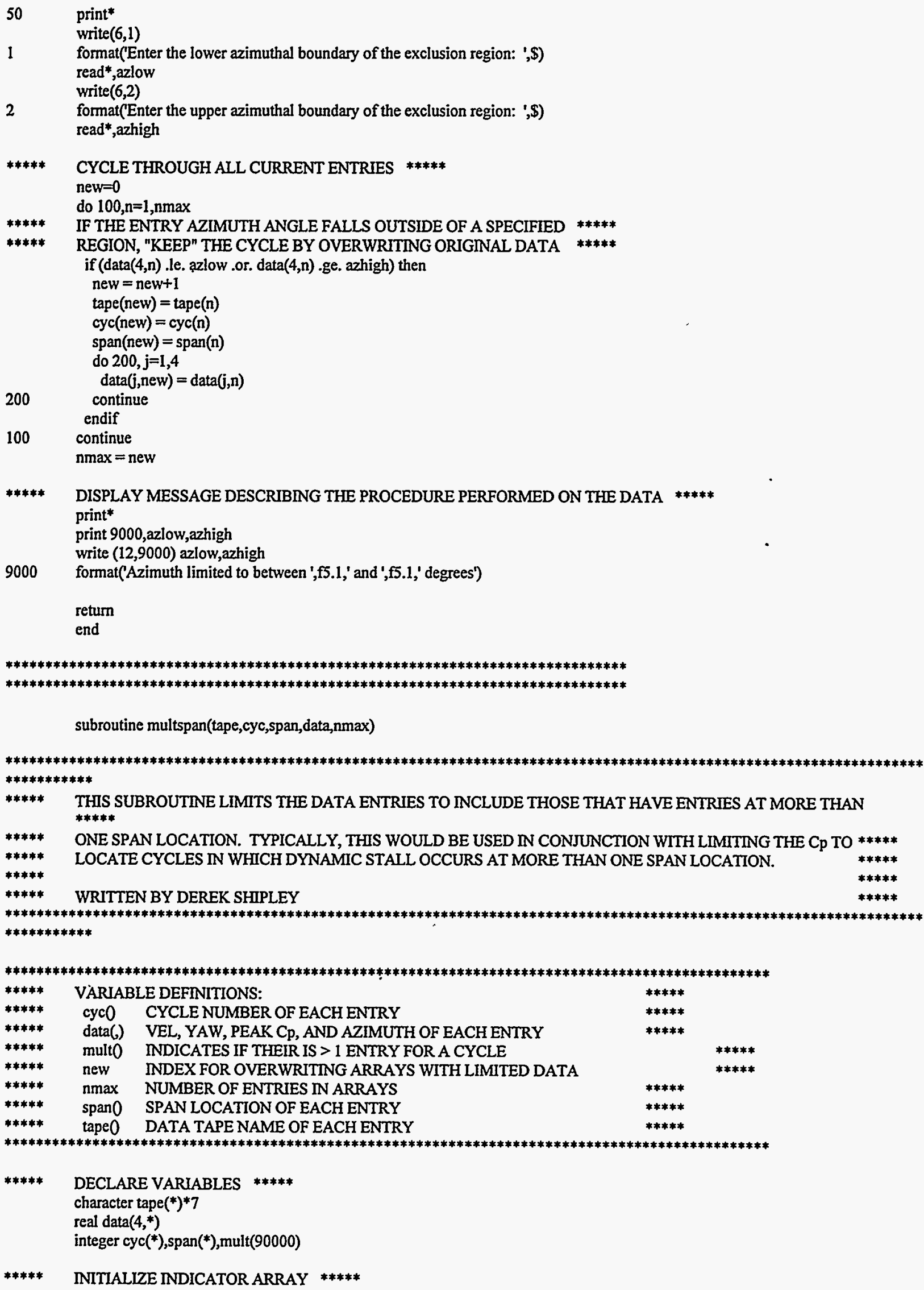


$\operatorname{read}^{*}$, opt

***** SET PROPER DATA COLUMNNUMBER AND OUTPUT MESSAGE

if (opt .eq. ' $V$ ' .or. opt .eq. ' $v$ ') then

ncol $=1$

sortcol $=$ 'velocity'

elseif (opt .eq. ' $\mathrm{Y}$ ' .or. opt .eq. ' $\mathrm{y}$ ') then

ncol $=2$

sortcol $=$ 'yaw'

elseif (opt .eq. ' $C$ ' .or. opt .eq. ' $c$ ') then

ncol $=3$

sortcol $=$ ' $\mathrm{Cp}$ '

else

print*,'Invalid option. Please try again.'

goto 5

endif

***** SET SORT VARIABLE EQUAL TO DESIRED DATA COLUMN *****

do $50, m=1, n$

$\mathrm{ra}(\mathrm{m})=\mathrm{data}(\mathrm{ncol}, \mathrm{m})$

50 continue

***** BEGN LOOP TO SORT DATA IN PLACE (HEAPSORT METHOD)

$I=n / 2+1$

ir $-n$

10 continue

if(l.gt.1)then

I $=\mathrm{I}-\mathrm{I}$

rra $=r a(l)$

rtape $=$ tape(l)

rcyc=cyc(l)

rspan $=$ span $(1)$

do $100, k=1,4$

$100 \quad \operatorname{rdata}(k)=$ data $(k, 1)$

else

па=-ra(ir)

rtape $=$ tape(ir)

rcyc $=$ cyc(ir)

rspan=span(ir)

do $200, k=1,4$

200

$\mathrm{a}(\mathrm{k})=\mathrm{data}(\mathrm{k}, \mathrm{i} r)$

$\mathrm{ra}(\mathrm{ir})=\mathrm{ra}(\mathrm{l})$

tape(ir)=tape(1)

cyc(ir) $=$ cyc $(1)$

$\operatorname{span}(\mathrm{ir})=\operatorname{span}(1)$

do $300, k=1,4$

300 continue

ir=ir-1

if(ir.eq.1)then

$\mathrm{ra}(\mathrm{l})=\mathrm{ma}$

tape(1)=rtape

$\operatorname{cyc}(1)=$ rcyc

$\operatorname{span}(1)=r s p a n$

do $400, k=1,4$

400

continue $\operatorname{data}(k, 1)=\operatorname{Idata}(k)$

print*

print 9000 , sortcol

write $(12,9000)$ sortcol

return

endif

endif

$\mathrm{i}=1$

$j=1+1$ 
endif

if(ra.lt.ra(j))then

$\mathrm{ra}(\mathrm{i})=\mathrm{ra}(\mathrm{j})$

$$
\text { tape(i)=tape(j) }
$$

$\operatorname{cyc}(i)=\operatorname{cyc}(i)$

$\operatorname{span}(\mathbf{i})=\operatorname{span}(\mathrm{j})$

- do $500, k=1,4$

500 continue $\operatorname{data}(k, i)=\operatorname{data}(k, j)^{-}$

$\mathrm{i}=\mathbf{j}$

$j=j+j$

else

$\mathrm{j}=\mathrm{ir}+1$

endif

go to 20

endif

$r a(i)=\pi a$

tape(i)=tape

$\operatorname{cyc}(i)=$ rcyc

$\operatorname{span}(\mathrm{i})=\operatorname{sspan}$

do $600, \mathrm{k}=1,4$

$\operatorname{data}(\mathrm{k}, \mathrm{i})=\mathrm{rdata}(\mathrm{k})$

600 continue

go to 10

9000 format('Data sorted in increasing order by ',al0)

end

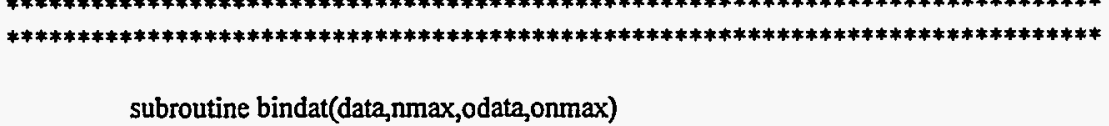

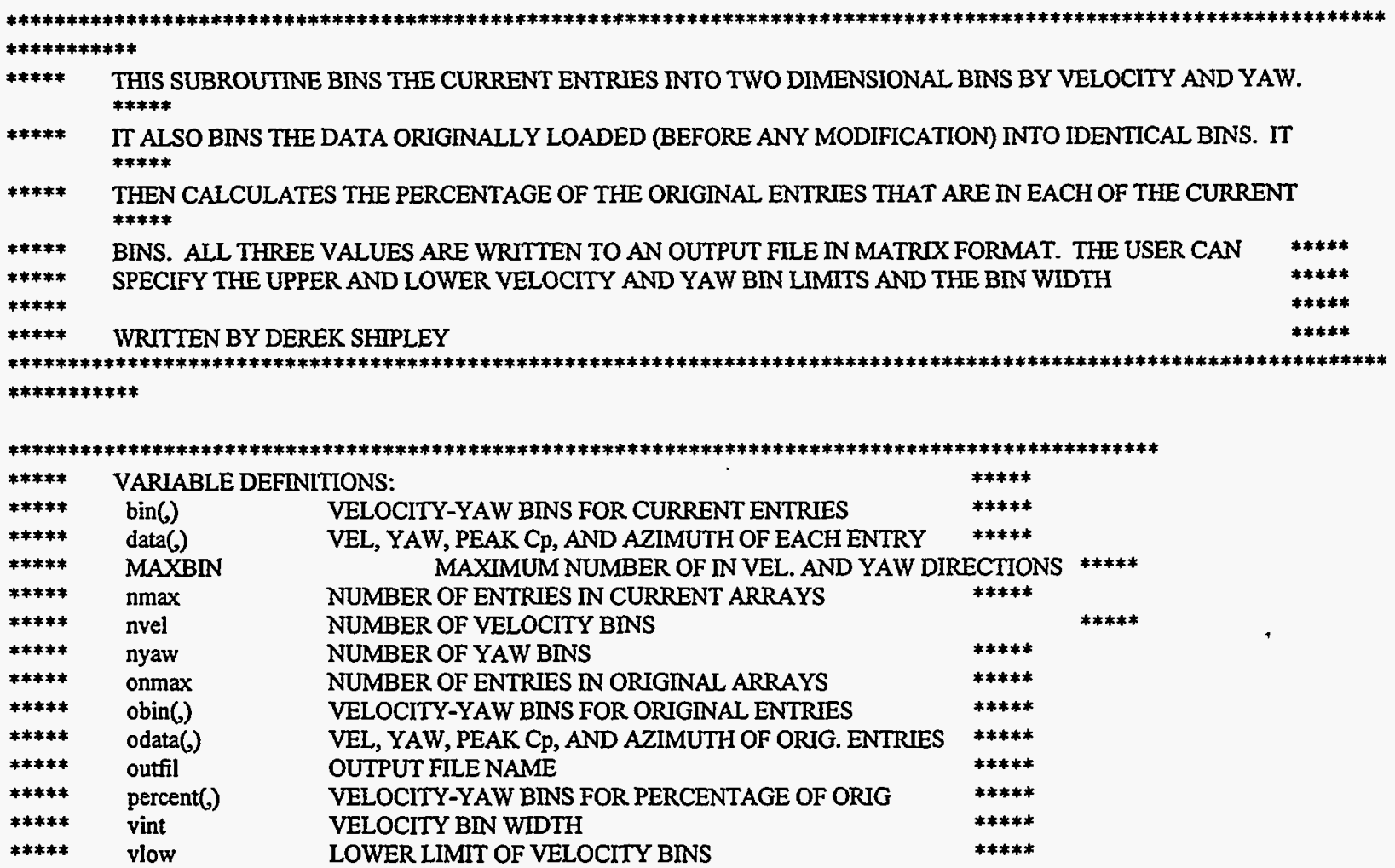




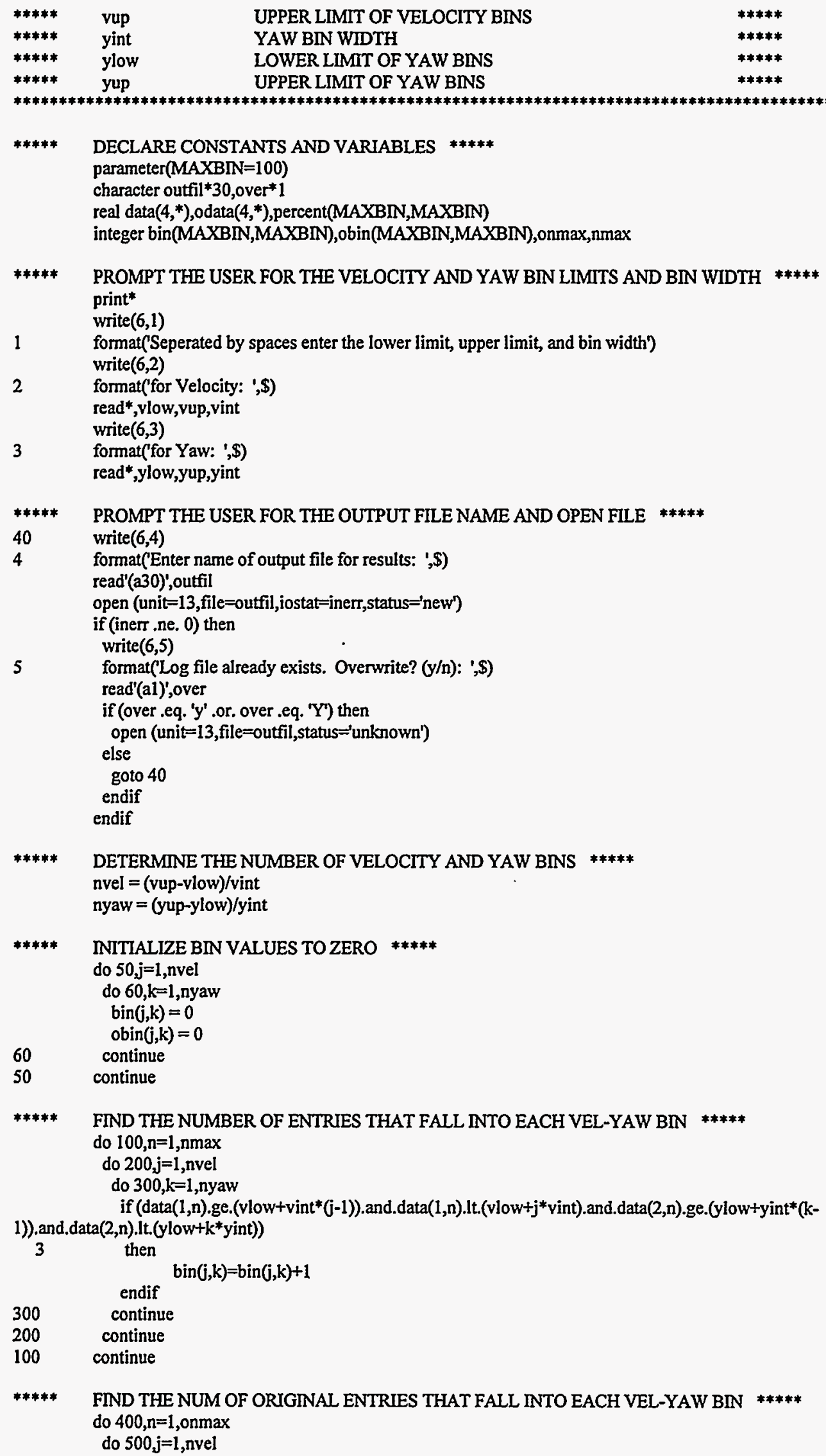




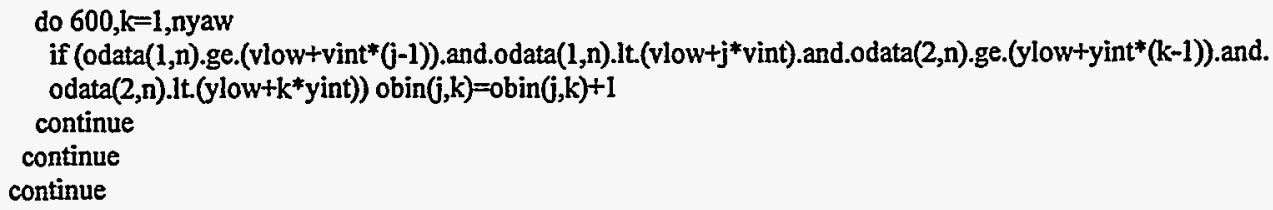

DETERMINE THE PERCENTAGE OF THE ORIGNAL ENTRIES THAT FALL INTO EACH VEL-YAW BIN do $700, \mathrm{j}=1$, nvel do $800, \mathrm{k}=1$, nyaw

if (obin(j,k).gt.0) then

percent $(j, k)=$ float $($ bin $(j, k)) / f l o a t(o b i n(j, k))^{*} 100$.

eise

percent $(j, k)=0$

endif

continue

700

continue

WRITE THE PERCENTAGES OF ORIGINAL ENTRIES IN EACH VEL-YAW BIN TO THE OUTPUT FILE write(13,9000)0,(ylow+(yint*(2.*k-1)/2.),k=1,nyaw)

do $900, j=1$,nvel

write $(13,9000)$ vlow+(vint*(2.*j-1)/2.),(percent(j,k), $\mathrm{k}=1$, nyaw)

***** WRTE THE NUMBER OF CURRENT ENTRIES IN EACH VEL-YAW BIN TO THE OUTPUT FILE ***** write $(13, *)$

write(13,9000)0,(ylow+(yint*(2.*k-1)/2.), $\mathrm{k}=1$, nyaw $)$

do $1000, j=1$,nvel

write(13,9100)vlow+(vint*(2.*j-1)/2.),(bin(j,k),k=1,nyaw)

1000 continue

***** WRITE THE NUMBER OF ORIGINAL ENTRIES IN EACH VEL-YAW BIN TO THE OUTPUT FILE ***** write $(13, *)$

write $(13,9000) 0,($ ylow+(yint* $(2 . * k-1) / 2),. k=1$, nyaw $)$

do $1100, \mathrm{j}=1$,nvel

write(13,9100)vlow+(vint*(2.*j-1)/2.),(obin(j,k),k=1,nyaw)

1100 continue

***** DISPLAY MESSAGE DESCRIBING THE PROCEDURE PERFORMED ON THE DATA *****

print*

print 9200 ,vlow, vup,ylow,yup

write $(12,9200)$ vlow,vup,ylow,yup

9200 format('Data binned from ',f4.1,' -',f4.1,' m/s and ',f5.1,' -',

2 f5.1,' deg')

close(13)

return

end

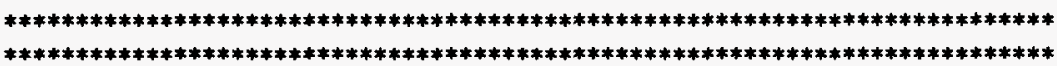

subroutine writdat(tape,cyc,span,data,nmax)

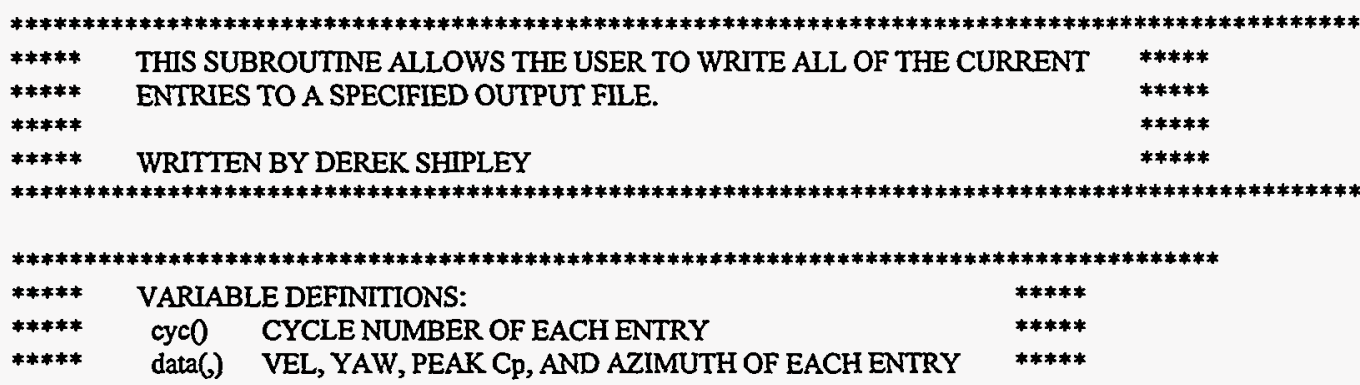




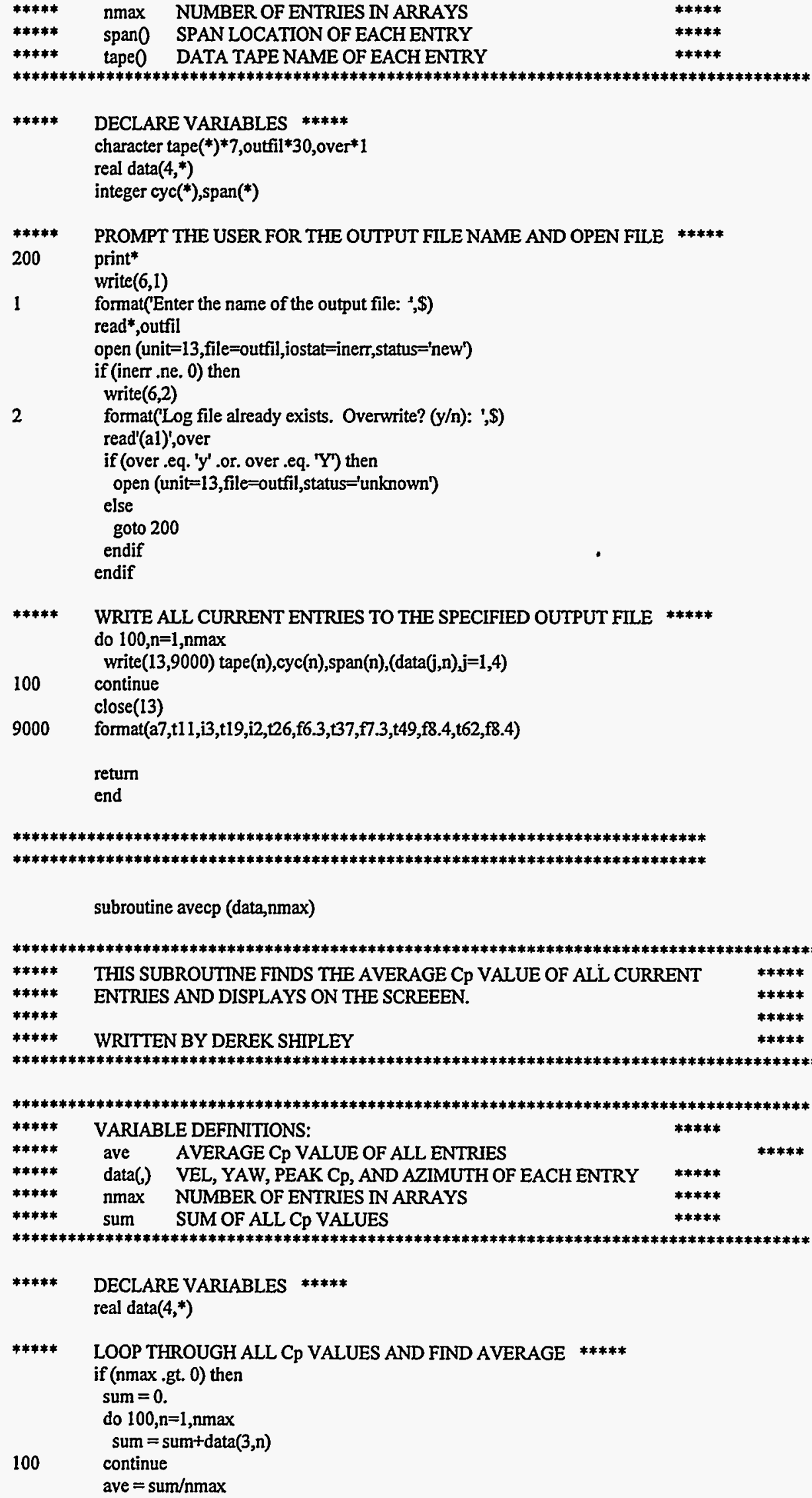


print*

if (nmax .It. 10) then

write $(6,9010)$ nmax,ave

write(12,9010)nmax,ave

elseif (nmax .It. 100) then

write $(6,9020)$ nmax, ave

write(12,9020)nmax,ave

elseif (nmax .lt. 1000) then

write $(6,9030)$ nmax,ave

write $(12,9030)$ nmax,ave

elseif (nmax .It. 10000) then

write $(6,9040)$ nmax,ave

write $(12,9040)$ nmax, ave

else

write $(6,9050)$ nmax,ave

write $(12,9050)$ nmax, ave

endif

else

write $(6,9100)$

write $(12,9100)$

endif

format(The average of ',il,' $C p$ values is ',f6.2)

format('The average of ', $i 2$, ' $C p$ values is ',f6.2)

format(The average of ', $i 3$, ' $C p$ values is ', $f 6.2$ )

format('The average of ',i4, $\mathrm{Cp}$ values is ',f6.2)

format(The average of',i5,' $\mathrm{Cp}$ values is ',f6.2)

format('There are no values to average')

return

end 
Public reporting burden for this collection of information is estimated to average 1 hour per response, including the time for reviewing instructions, searching existing data sources, gathering and maintaining the data needed, and completing and reviewing the collection of information. Send comments regarding this burden estimate or any other aspect of this collection of information, including suggestions for reducing this burden, to Washington Headquarters Services, Directorate for Information Operations and Reports, 1215 Jefferson Davis Highway, Suite 1204, Arlington, VA 22202-4302, and to the Office of Management and Budget, Paperwork Reduction Project (0704 0188 , Washington, DC 20503.

1.

2. REPORT DATE November 1995
3. REPORT TYPE AND DATES COVERED Subcontract Report
4. TITLE AND SUBTITLE

Combined Experiment Phase II Data Characterization

6. AUTHORIS

Mark S. Miller, Derek E. Shipley, Teresa S. Young, Michael C. Robinson, Marvin W. Luttges, David A. Simms

7. PERFORMING ORGANIZATION NAME(S) AND ADDRESS(ES)

University of Colorado at Boulder

Campus Box 19

Boulder, CO 80309

National Renewable Energy Laboratory

1617 Cole Boulevard

Golden, CO 80401-3393

9. SPONSORING/MONITORING AGENCY NAME(S) AND ADDRESS(ES)

National Renewable Energy Laboratory

1617 Cole Blvd.

Golden, CO 80401-3393
5. FUNDING NUMBERS

C:

TA: WE618110

8. PERFORMING ORGANIZATION REPORT NUMBER

\section{SUPPLEMENTARY NOTES}

12a. DISTRIBUTION/AVAILABILITY STATEMENT National Technical Information Service

U.S. Department of Commerce

5285 Port Royal Road

Springfield, VA 22161 12b. DISTRIBUTION CODE

UC-1211

\section{ABSTRACT (Maximum 200 words)}

The National Renewable Energy Laboratory's "Combined Experiment" has yielded a large quantity of experimental data on the operation of a downwind horizontal axis wind turbine under field conditions. To fully utilize this valuable resource and identify particular episodes of interest, a number of databases were created that characterize individual data events and rotational cycles over a wide range of parameters. Each of the $\mathbf{5 9}$ five-minute data episodes collected during Phase II of the Combined Experiment have been characterized by the mean, minimum, maximum, and standard deviation of all data channels, except the blade surface pressures. Inflow condition, aerodynamic force coefficient, and minimum leading edge pressure coefficient databases have also been established, characterizing each of nearly 21,000 blade rotational cycles. In addition, a number of tools have been developed for searching these databases for particular episodes of interest. Due to their extensive size, only a portion of the episode characterization databases are included in an appendix, and examples of the cycle characterization databases are given. The search tools are discussed and the FORTRAN or $\mathrm{C}$ code for each is included in appendices.

\section{SUBJECT TERMS}

horizontal axis wind turbines; wind turbine aerodynamics

15. NUMBER OF PAGES

16. PRICE CODE

20. LIMITATION OF ABSTRACT

OF THIS PAGE

Unclassified
19. SECURITY CLASSIFICATION OF ABSTRACT Unclassified
UL 
